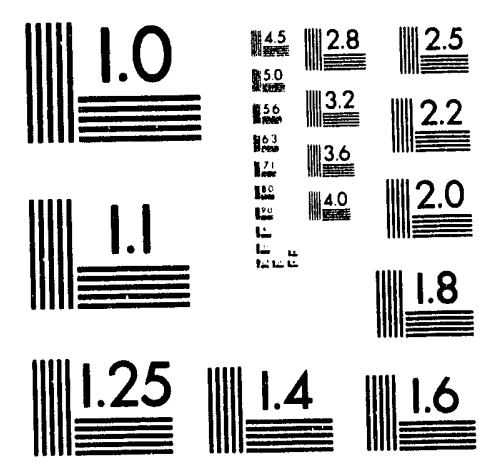



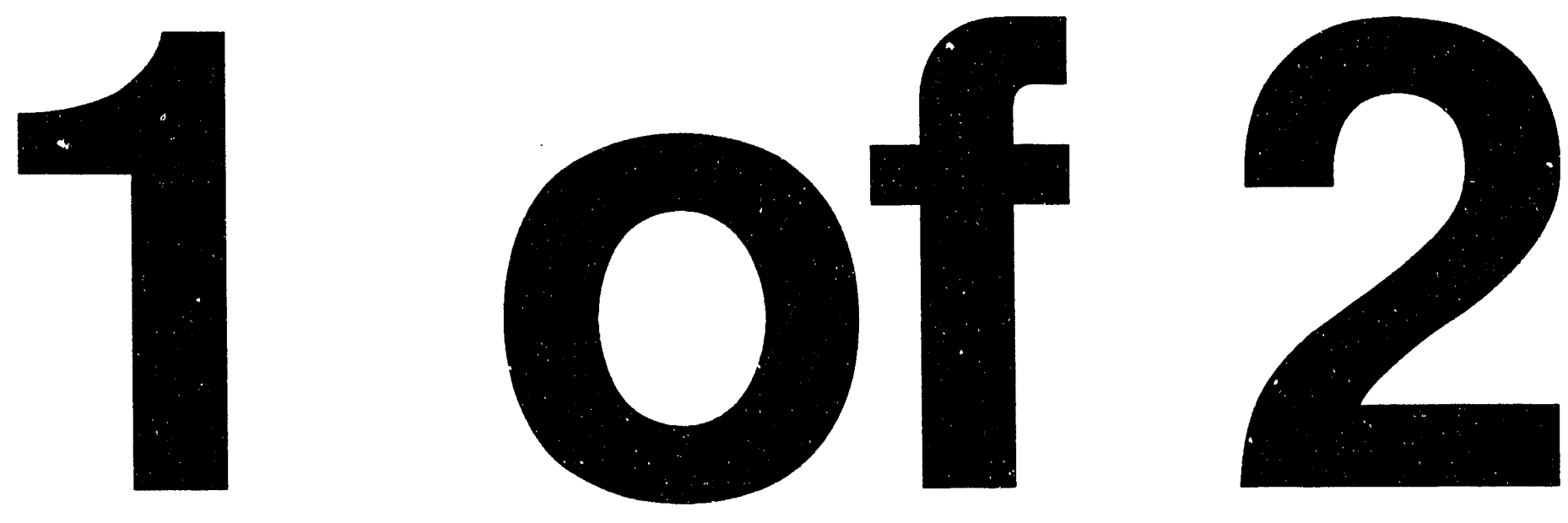
Metals and Ceramics Division

\begin{abstract}
DEVELOPMENT OF IMPROVED PROCESSING AND EVALUATION METHODS FOR HIGH REIIABIIITY STRUCTURAL CERAMICS FOR ADVANCED HEAT ENGINE APPLICATIONS, PHASE I

Vimal K. Pujari, Dennis M. Tracey, Michael R. Foley, Norman I. Paille, Paul J. Pelletier, Lenny C. Sales, Craig A. Wilkens, and Russell I. Yeckley
\end{abstract}

Date Published: August 1993

EINAL REPORT

\author{
Prepared by \\ Norton Company \\ Advanced Ceramics \\ Goddard Road \\ Northboro, Massachusetts 01532 \\ Under Subcontract $86 \mathrm{X}-\mathrm{SB} 182 \mathrm{C}$
}

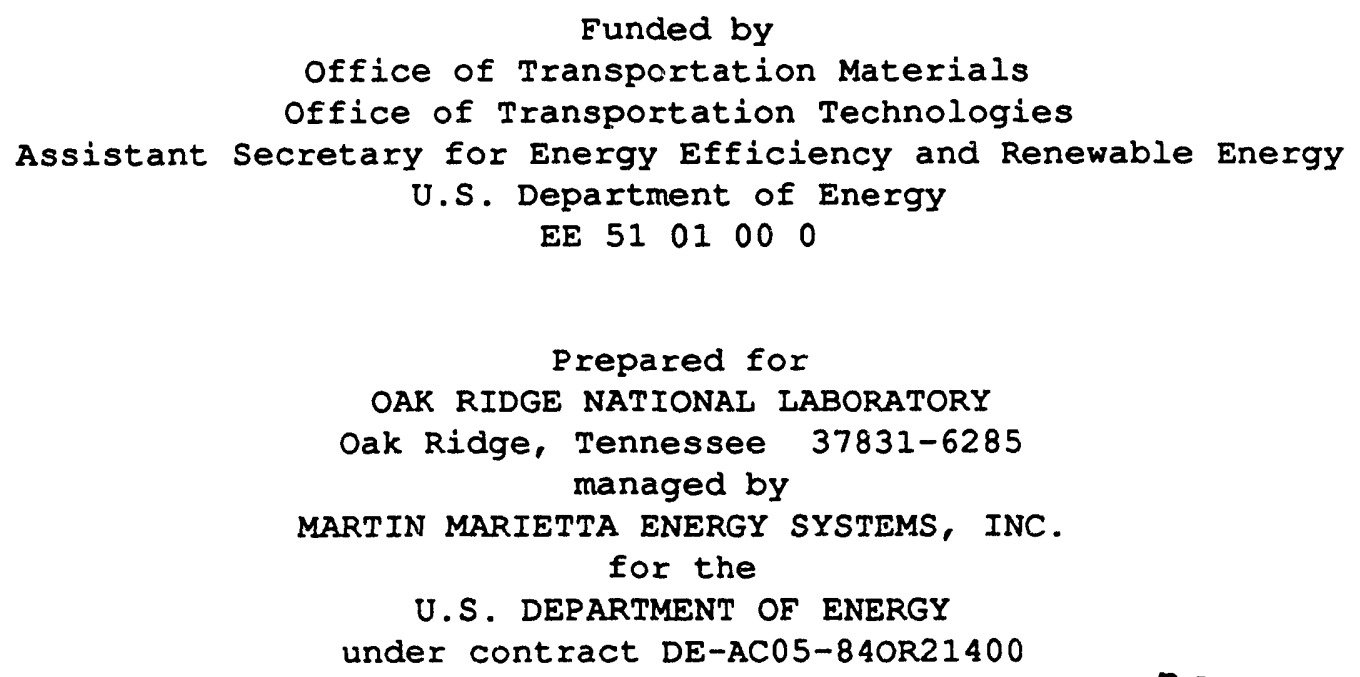


EXECUTIVE SUMMARY . . . . . . . . . . . . . . . . . . . iv IIST OF TABLES . . . . . . . . . . . . . . . . . . . . . . x IIST OF FIGURES . . . . . . . . . . . . . . . . . . . . . . . . . . xi ABSTRACT . . . . . . . . . . . . . . . . . . . . 1 INTRODUCTION . . . . . . . . . . . . . . . . . . . . . 2 COLLOIDAL CONSOLIDATION PROCESS . . . . . . . . . . . . . . . 4 POWDER PROCESSING . . . . . . . . . . . . . . . . . . . . . . 4 SLURRY DEVELOPMENT AND CHARACTERIZATION . . . . . . . . . 5 CASTING OF NET SHAPED FORMED (NSF) TENSILE BARS . . . . . . . . 6 HOT ISOSTATIC PRESSING .................... 7 MACHINING PROCESS OPTIMIZATION . . . . . . . . . . 8 TENSILE STRENGTH DATABASE . . . . . . . . . . . . . . . . . . . . 9 ROOM TEMPERATURE TENSILE TEST DATA . . . . . . . . . . . . 9 ELEVATED TEMPERATURE TENSILE TEST DATA . . . . . . . . . . . . 14 LARGE CROSS SECTION SPECIMEN STRENGTH DATA . . . . . . . . . . 17 CONCLUSIONS . . . . . . . . . . . . . . . . . . . . . . . 18 ACKNOWLEDGEMENTS . . . . . . . . . . . . . . . . . . 19 REFERENCES ............................ 20 PROJECT PRESENTATIONS . . . . . . . . . . . . . . . . . 21 PROJECT PUBLICATIONS . . . . . . . . . . . . . . . . 23

APPENDIX 1: MATERIAL SELECTION AND CHARACTERIZATION . • . . . . . 25 MATERIAL SELECTION . . . . . . . . . . . . . . . . 25 MATERIAL CHARACTERIZATION . . . . . . . . . . . . . . 25 oxygen Analysis... . . . . . . . . . . . . . . 26 Yttria and oxygen values . . . . . . . . . . . . . 26 Analysis of Metallic species . . . . . . . . . . 26 Particle size and Surface Area of the Powder . . . . . 27 Rheological Measurements . . . . . . . . . . 27 IEP Measurements . . . . . . . . . . . . . . . 27 Milling media . . . . . . . . . . . . .... . 28 CHARACTERIZATION OF THE MILLED SILICON NITRIDE SLURRIES . • . 28 CHARACTERIZATION OF INJECTION MOLDING BINDER . . . . . . . . 31 CHARACTERIZATION OF CASTING PLASTER MOLDS . . . . . . . . . 31

APPENDIX 2: MATERIAL PROCESSING AND PROCESS CONTROI . . . . . . . 32 POWDER PROCESSING .................... 32 W-Series Process .................. . 33 C-Series Process ................. . 33

PROCESS CONTROL ....................... 36 sQC Control charts ................ 36 Quality Assurarce . . . . . . . . . . . . . . 36 Milling Control ................... . 37 surface Chemistry . . . . . . . . . . . . 38 Inclusion/Agglomerate Free Product . . . . . . . . 40 Milling optimization ............... . 40 COLLOIDAI CONSOLIDATION PROCESS DEVELOPMENT . . . . . . . . 42 slurry Development and Characterization . . . . . 42 
Mold Design ..................... 44

Density Uniformity Control During Filtration . . . . 45

Casting of Net Shape Formed (NSF) Tensile Bars . . . . 47

Ultrasonic Monitoring of the Casting Process . . . . . 49

Ultrasonic Characterization of the Ceramic

suspension. . . . . . . . . . . . . . 49

In-Situ Monitoring of the Filtration

Process . . . . . . . . . . . . . . . 49

Comparison of Ultrasonic and Physical Casting

Rate Data . . . . . . . . . . . . . . 51

INJECTION MOLDING PROCESS DEVELOPMENT ............. 51

Compounding . . . . . . . . . . . . . . . . 52

Mold Design . . . . . . . . . . . . . . . . 52

Molding Process optimization and control ...... . 53

Dewaxing . . . . . . . . . . . . . . . 55

HOT ISOSTATIC PRESSING . . . . . . . . . . . . . . . . 56

HIP Process Development . . . . . . . . . . . . 56

Microstructural Development . . . . . . . . . . 58

REFERENCES ......................... . 62

APPENDIX 3: DEVELOPMENT AND APPLICATION OF NDE METHODS . . . . . . 63

TECENIQUE DEVELOPMENT . . . . . . . . . . . . . . . . . . 63

INJECTION MOLDING PROCESS NDE SUPPORT . . . . . . . . . . 65

COLLOIDAL CONSOLIDATION - BASELINE ITERATION - NDE SUPPORT • 70

STAGE II PROCESS OPTIMIZATION NDE SUPPORT . . . . . . . . 71

As-Received Powder ............... . 71

Colloidal suspensions... . . . . . . . . . . 72

Presintered NSF Bars . . . . . . . . . . . . . . 72

HIP'ed NSIE Bars . . . . . . . . . . . . . . . 73

Density Gradient Evaluation . . . . . . . . . . . 74

Strength-Flaw Correlation . . . . . . . . . . 75

Optimizing X-ray Unit Performance . . . . . . . . . 76

Detection of Agglomerates in Dense NSF Bars . . . . . 77

STAGE III PROCESS DEMONSTRATION NDE SUPPORT . . . . . . . . 78

In-Process Inspection . . . . . . . . . . . . 78

Radiography Data for Machined NSF Bars . . . . . . . 81

Liquid Dye Penetrant Data for Machined NSF Bars . . . 82

REFERENCES . . . . . . . . . . . . . . . . . . 86

APPENDIX 4: PROPERTY TESTING AND MICROSTRUCTURAL EVALUATION . • • • . 87

TESTING PROCEDURES . . . . . . . . . . . . . . . . . . 88

Dense Tensile Testing... . . . . . . . . . . 88

Green Tensile Testing . . . . . . . . . . . . 89

BASELINE TENSILE DATA . . . . . . . . . . . . . . . 89

Injection Molding . . . . . . . . . . . . . 89

Colloidal Consolidation ............... 90

BASELINE PRESSURE CASTING VERIFICATION - ITERATION N . . . . 94

MACHINING ISSUES . . . . . . . . . . . . . . . . . 96

Machining Related Buttonhead Failure . . . . . . 98

Fractography - Strength Correlation . . . . . . . 99 


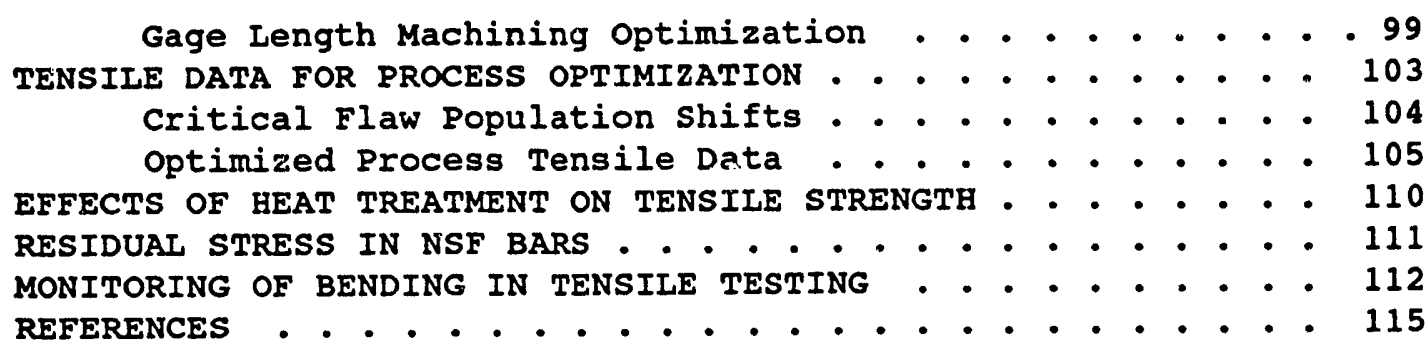

APPENDIX 5: TENSILE STRENGTH - FRACTOGRAPHY DATABASE • • • . . 116

Research sponsored by the U.S. Department of Energy, Assistant Secretary for Energy Efficiency and Renewable Energy, office of Transportation Technologies, as part of the Ceramic Technology Project of the Materials Development Program, under contract DE-AC05-840R21400 with Martin Marietta Energy Systems, Inc., Work Breakdown Structuse Subelement 1.1.4.1. 


\section{EXECUTIVE SUMMARY}

This report documents the accomplishments of an extensive four year Processing for Reliability R\&D program conducted at Saint-Gobain/ Norton Industrial Ceramics Corporation's Northboro Research \& Development Center and funded by the Department of Energy through Martin Marietta Energy systems, Inc. The program goals were to develop and demonstrate significant improvements in processing methods, process controls and non-destructive evaluation (NDE) which can be commercially implemented to produce high reliability silicon nitride components for advanced heat engine applications at temperatures to $1370^{\circ} \mathrm{C}$.

The program goals were addressed by focusing on a Si3N4-48 Y2O3

high temperature ceramic composition and hot- isostatic-pressing (HIP'ing) as the method of densification. Quantitative mechanical property targets for tensile strength and stress rupture performance were established and were used as decision point criteria during the course of the program. The plan called for the development of colloidal methods to allow high quality near net shape forming of a reasonably broad range of green body geometries. The geometry chosen for demonstration was the variable diameter buttonhead tensile rod which served to define a suitably challenging forming requirement. The tensile rod : : $165 \mathrm{~mm}$ long with $16 \mathrm{~mm}$ "buttonhead" end grip sections and a $35 \mathrm{~mm}$ central gage length with $6 \mathrm{~mm}$ diameter. Besides being a suitable forming geometry, through tensile testing, the tensile rod afforded a direct means to assess product quality relative to the strength and stress rupture targets. Furthermore, the resultant property database provides an invaluable resource for future engineering design efforts which utilize advanced silicon nitride material.

The program was conducted by performing a series of complete process cycles (iterations) - powder processing, forming, HIP'ing, machining - with each cycle producing about 75 tensile rods. Each iteration served as an experiment in a test matrix which was designed with the key process parameters as variables. After the period of process experimentation, the plan called for a demonstration of the optimum process involving production and testing of over 300 tensile rods. The goal was to have the mean room temperature tensile strength of specimens from this final set equal to $900 \mathrm{MPa}$ with a variability equivalent to a Weibull distributior with threshold stress of $0 \mathrm{MPa}$ and shape factor (modulus) of 20. The elevated temperature goals were a mean $1370^{\circ} \mathrm{C}$ tensile strength of $500 \mathrm{MPa}$ and a $1230^{\circ} \mathrm{C}, 350 \mathrm{MPa}$ tensile stress rupture life of 100 hours.

The program was conducted following six major tasks, Table I, each of which was pursued according to requirements and milestones specified within the contract statement of work. The program plan involved three successive stages of processing research. stage I was conducted over the first two year period and had as major objectives : 1! comparing injection molding and colloidal consolidation process routes, and selecting one route for subsequent optimization, 2) comparing tine performance of water milled and alcohol milled powder and selecting one on the basis of performance data, and 3) adapting several NDE methods to 
the needs of ceramic processing. The NDE methods considered were microfocus $x$-ray radiography, computed tomograwhy, ultrasonics, NMR imaging, NMR spectroscopy, fluorescent liquid dye penetrant and $x$-ray diffraction residual stress analysis.

Table I: Major Tasks in the Program

\begin{tabular}{||lll||}
\hline & & \\
- TASK 1: & Material Selection and Characterization \\
- & TẢSK 2: & Material Processing and Process Control \\
- TASK 3: & Development and Application of NDE \\
- TASK 4: & Property Testing and Microstructural Evaluation \\
- TASK 5: & Reporting \\
\hline
\end{tabular}

A requirement for conclusion of stage $I(1 / 31 / 91)$ was a comparison of databases generated in the Injection Molding (IM) and colloidal Consolidation (CC) baseline iterations. On the kasis of this comparison, it was required to select one of the two forming techniques for the remainder of the program, with the approval of the ORNL technical monitor.

Data obtained from the forming and testing of net shape formed (NSF) tensile rods and $50 \mathrm{~mm}$ diameter cylindrical (MLP) specimens were used in the comparison of the IM and CC processes. Specific data used in this comparison included: 1) yield at each major processing sub-step, 2) total process yie.ld, 3) total process cycle time, 4) green Hensity uniformity, 5) room temperature average tensile strength and Weibull modulus froin tensile testing iteration NSF specimens, 6) high temperature average tensile strength from NSF specimens, 7) fracture toughness from MOR flexure bars machined from NSF specimens, 8) stress rupture data from MOR flexure bars machined from NSF specimens, and 9) formability of MLP specimens.

The data which favored IM relative to $C C$ were the total process yield and the uniformity of green density. CC on the other hand was found to offer a considerably shorter total process cycle time, significantly greater averages for room and elevated temperature tensile strength and large section (MIP) formability in the sense of demonstrated capability to process specimens free of macroscopic drying/dewaxing cracks.

The overriding goal of the program was processing methodology capable of producing specimens with an average tensile strength of 900 MPa and high reliability, which is adaptable to large cross-sections, such as that of the MUP specimens. The CC forming technique had the advantage in both respects, on the basis of these iteration data. The baseline CC room temperature average tensile strength (692 MPa) was 528 higher than that of the IM route (454 MPa). Furthermore, several CC specimens had strengths in excess of the $900 \mathrm{MPa}$ goal, with one being in excess of $1000 \mathrm{MPa}$. In comparison, the maximum strength recorded in the 
IM iteration was $663 \mathrm{MPa}$. Formability was found to be a serious problem for larger cross-sections using the IM process. Optimal conditions of the dewax unit operation for MLP specimens were not achieved and cracking during dewaxing remains as a technical barrier in need of resolution for large cross-sections.

In the basis of demonstrated attainable strength and MLP formability, the colloidal consolidation process route was selected and approved as the forming technique for the remainder of the program. The issues of low yield and low Weibull modulus found in the baseline iteration were to be addressed at the beginning of the stage II effort.

Eighteen process iterations were completed during stage II over a 15 month period. Table II provides a strategiral view of the effort relative to the three areas of emphasis: processing methods, process control and NDE. The outcome of the processing research was an environmentally sound, closed loop colloidal processing methodology. The process starts with aqueous vibration milling using $\mathrm{Si}_{3} \mathrm{~N}_{4}$ milling media, followed by magnetic separation, filtration and dilution water removal. There is no need to dry the milled powder so uninterrupted colloidal processing from milling to casting is achieved, which translates into significant reduction in common types of contamination.

Table II: Process Optimization Strategy
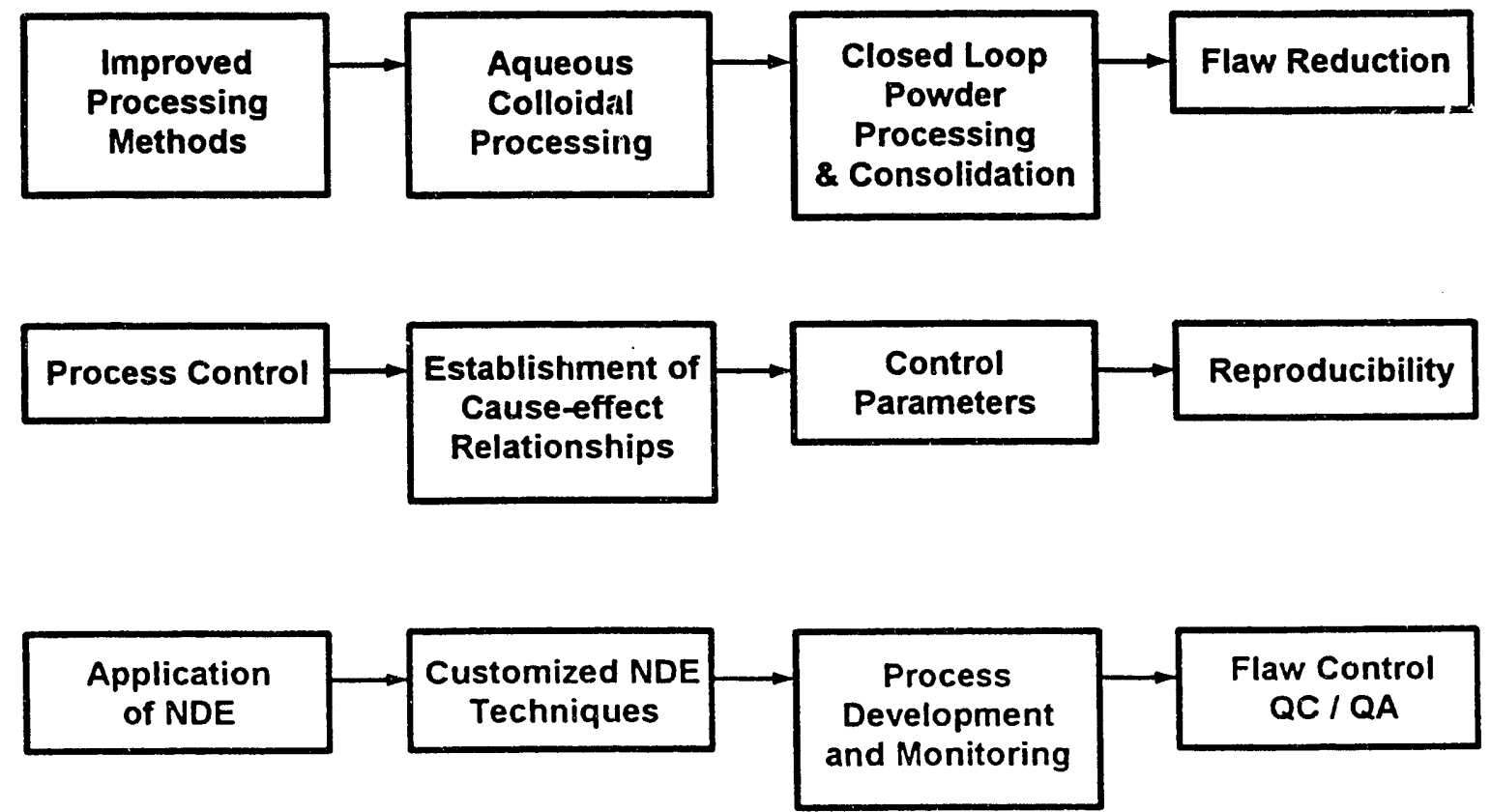

The pricess control research employed experimental design methods to establish cause-effect relationships involving key process variables identified during stage $I$. Control parameters were defined during the 
course of this iterative experimentation and statistical process control (SPC) methods were implemented to assure process reproducibility. Notable examples of innovation include a method for control of the colloidal consolidation process (pressure casting operation) for formation of a uniform density green microstructure, BIP cycle definition which assures a high toughness acicular grain microstructure at greater than 99.58 theoretical density, and a machining methodology for the cylindrical tensile bars which maintained control of strength impairing surface damage.

NDE methods were customized during stage II to provide novel characterization tools found necessary in the effort to establish causeeffect relationships. NDE played a crucial role as well in the areas of process monitoring and in-process flaw detection.

The advances in process technology realized during stage II are reflected in statistics of flaw detection obtained by microfocus $x$-ray radiography (MFX). MFX data on average size and frequency of defects in HIP'ed tensile bars for the two baseline iterations and several stage II iterations are plotted in Figure I. These data represent a grouping of results for pores, inclusions and agglomerates detected in the gage length volume. clearly, dramatic improvements are suggested by these results.

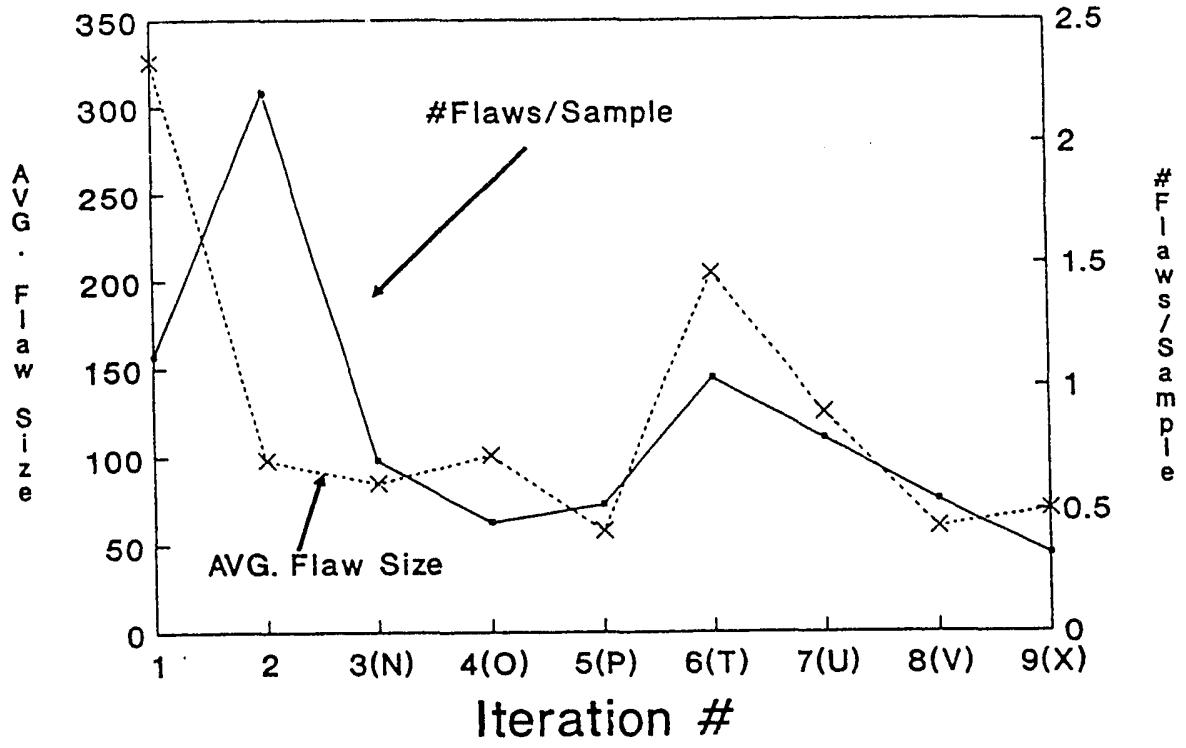

Figure I: Flaw Statistics from MFX Evaluation of Iteration samples

The material produced by the final stage II optimized process has been given the designation NCX-5102 silicon nitride. According to plan, a large number of specimens were produced and tested during stage III to establish a statistically robust room temperature tensile strength database for this material. Six process cycles were needed to produce the necessary set of approximately 400 tensile bars. These cycles followed the SOP established during stage II. The fabrication and testing effort was accomplished during the seven month period May 
through November, 1992.

The process demonstration provided experience and data which suggest that NCX-5102 truly ranks as a world class structural silicon nitride material. The test program established a mean tensile strength of $997 \mathrm{MPa}$ for the set of 320 tensile rods which fractured within the one cubic centimeter uniformly stressed gage section. More than 808 of the samples had strengths above the target value of $900 \mathrm{MPa}$ and approximately 508 had values in excess of $1 \mathrm{GPa}$, as is evident from the strength data histogram, Figure II.

The strength distribution for the sample set was determined by SEM fractographic analysis to be multimodal with several flaw populations contributing to the strength defining fast fractures. The range of the data was from a low value of $540 \mathrm{MPa}$ to a peak value of $1237 \mathrm{MPa}$. A 2parameter Weibull fit of the complete data set without the necessary competing risk analysis suggests a Weibull modulus of 10.4 . However, competing risk data analysis suggests that the strength distribution associated with process related, intrinsic strength impairing flaws is best represented instead by a 3-parameter Weibull model, Figure III. Approximately one-half of the data fall into this category. The other half primarily involved machining damage related failure origins and define a distinctly different strength distribution.

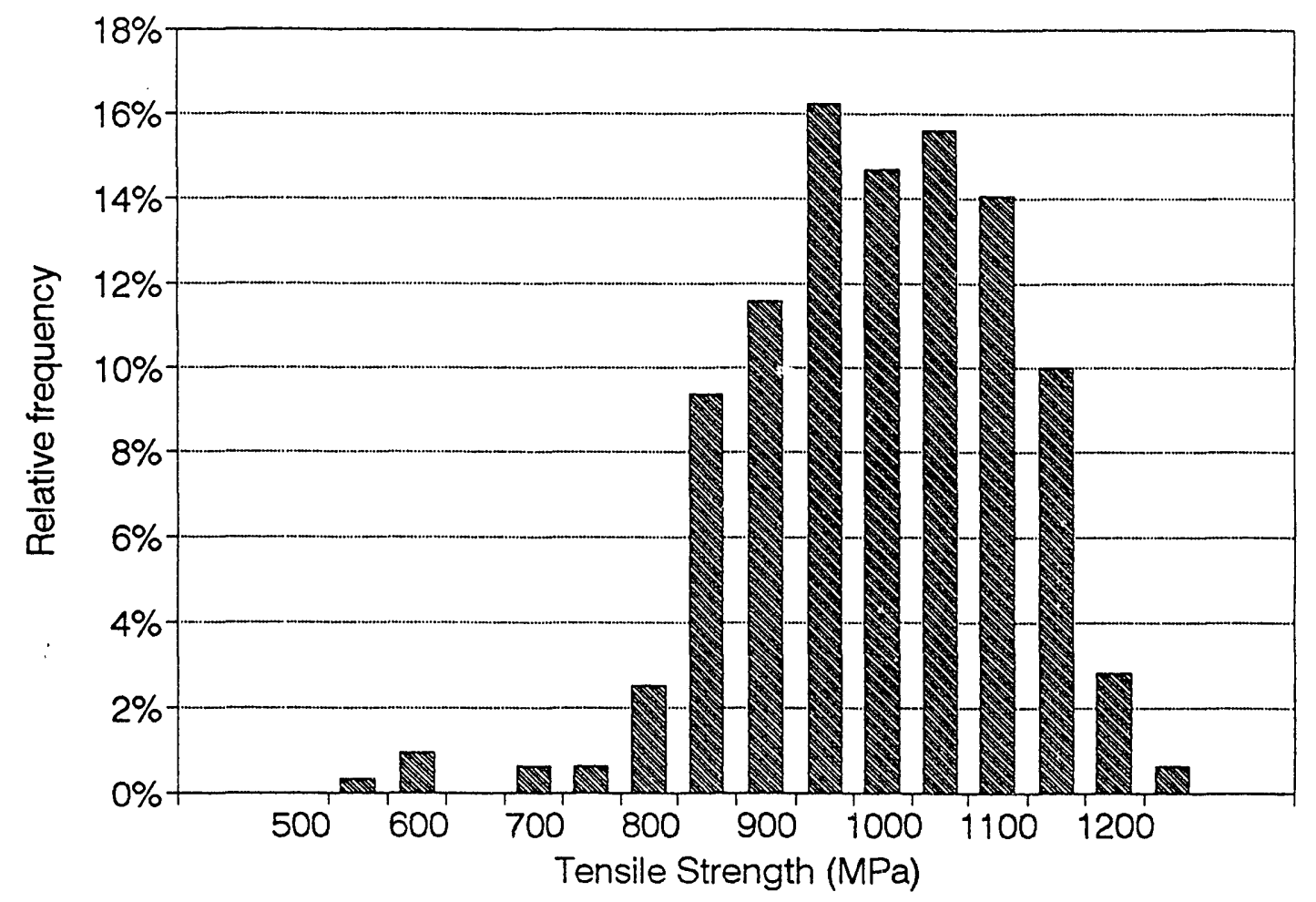

Figure II: Histogram of 320 Stage III Tensile Strength Data

The intrinsic strength distribution has a threshold strength equal to $665 \mathrm{MPa}$ which suggests that $\mathrm{NCX}-5102$ is intrinsically more reliable 
than the program target material envisioned to have a (2-parameter) Weibull modulus of 20 .

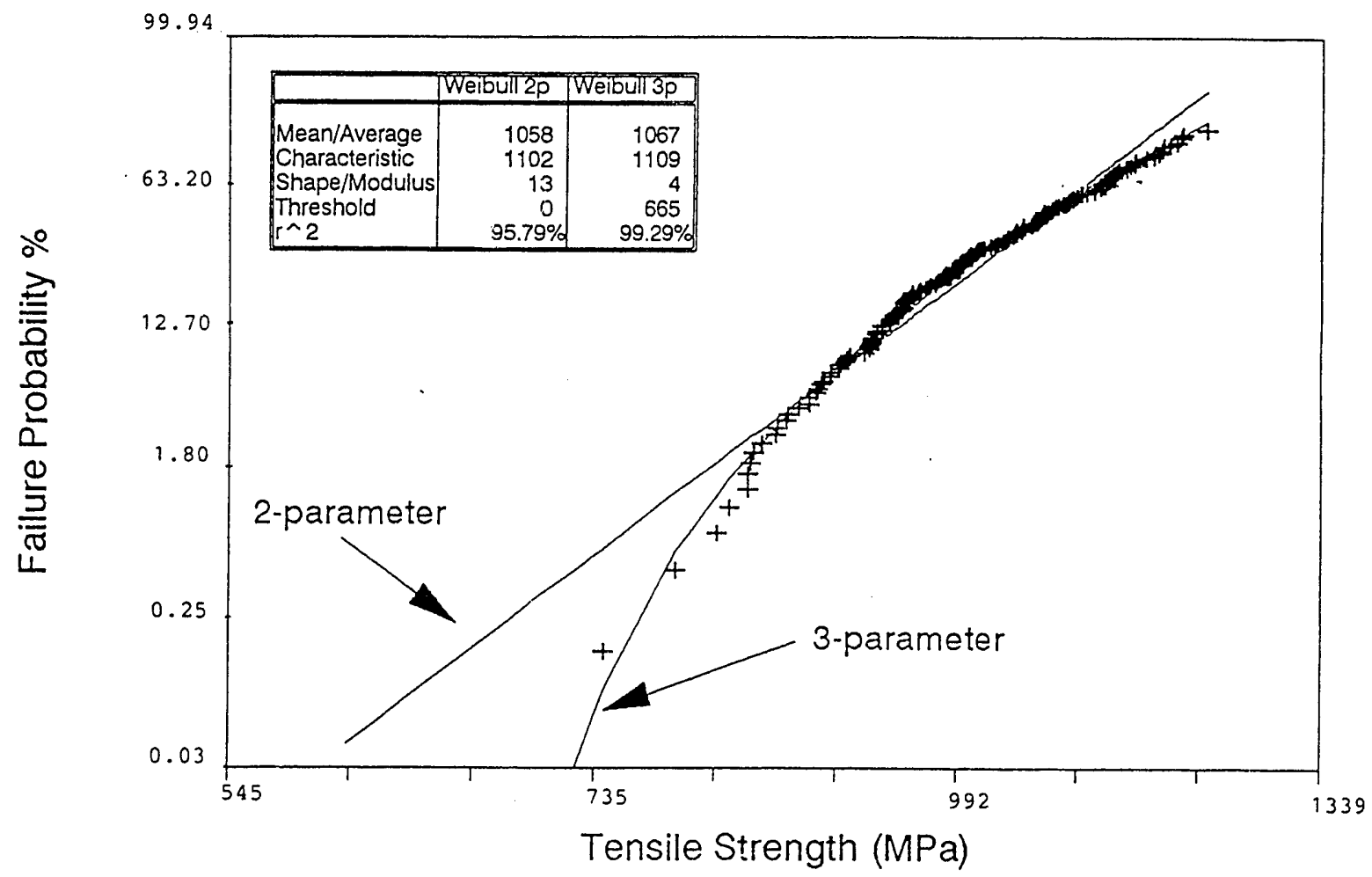

Figure III: NCX-5102 Cumulative Probability of Failure for Intrinsic Tensile Strength Data from Competing Risk Analysis.

Highlights of the Stage III process demonstration and resultant database are included in the main text of the report, along with a synopsis of the NCX-5102 aqueous based colloidal process. The R\&D accomplishments for Tasks 1-4 are discussed in detail in Appendices 1-4, while the tensile strength - fractography database for the stage III NCX-5102 process demonstration is provided in tabular form in Appendix 5. These appendices serve to supplement the main text and provide a complete account of the Processing for Reliability program.

Thus, in summary, the following has been attained after this four year advanced processing R\&D effort. A material (NCX-5102) having reliability significantly better than program goals and tensile strength at the $1 \mathrm{GPa}$ level with fracture toughness approaching $7 \mathrm{MPaVm}$ has been developed using an innovative closed loop aqueous process operation. In addition, an unprecedented tensile strength database for the advanced ceramic material silicon nitride has been established and is available for engineering/design utilization. Finally, the validity of in-process use and relative power of all the currently available NDE methods has been shown for the efficient manufacture of high quality advanced ceramic materials. 
I: Major Tasks in the Program . . . . . . . . . . . . . . . . . v

II: Process optimization strategy . . . . . . . . . . . . . . . . . vi

1: Stage III Fractography $\left(25^{\circ} \mathrm{C}\right)$ summary . . . . . . . . . . . . . 12

2: $\quad$ Probability of Survival at Given Stresses (MPa) . . . . . . . 14

2-1: Process Control Variables . . . . . . . . . . . . . 36

2-2: Powder Characterization Techniques . . . . . . . . . . . . 37

2-3: Ultrasonic Characteristics of Casting systeris . . . . . . . . . 49

2-4: Ceramic Molding Compound Material Property Data . . . . . . 54

2-5: Comparison of HIP Procedures . . . . . . . . . . . . . . . 57

2-6: Summary of HIP Results . . . . . . . . . . . . . . . . . . 57

2-7: Toughness Variability Observed with Batch V . . . . . . . . . 59

4-1: Tensile Data From NT154 Samples . . . . . . . . . . . . . . 88

4-2: Injection Molding Baseline RT Tensile Strength Data . • • • . 89

4-3: Injection Molding Baseline $1370^{\circ} \mathrm{C}$ Tensile strength Data . . . 90

4-4: Pressure Cast RT Baseline Tensile Strength Data . . . . . . . 91

4-5: slip Comparison for Pressure Cast silicon Nitride . . . . . . 92

4-6: Buttonhead Radius Surface Finish - Failure Data . . . . . . 98

4-7: Procedure Steps for Machining Tensile Rods. . . . . . . . . 103

4-8: Machining Procedure strength Data. . . . . . . . . . . 103

4-9: RT Tensile Strength Data for Batches XXX - Z . . . . . . 105

4-10: Fractography summary for Batches XXX - Z . . . . . . . . 105

5-1: NCX-5102 Database Summary . . . . . . . . . . . . . . . 116

5-2: NCX-5102 Database . . . . . . . . . . . . . . . 117 
IIST OF FIGURES

I: Flaw statistics from MF Evaluation of Iteration samples . . vii

II. Bistogram of 320 stage III Tensile strength Data . . . . . viii

IIi: NCX-5102 Cumulative Probability of Failure for Intrinsic Tensile strength Data from Competing Risk Analysis . . . . . . ix

1: Comparison of Aqueous and Alcohol Based Powder Processing Methods . . . . . . . . . . . . . . . . . 4

2: Surface Area for Various Grades and Lots of UBE Silicon Nitride Powder . . . . . . . . . . . . . . . . 5

3: Zeta Potential vs. pH for Si3N4 Lots D and E . . . . . . . . . 5

4: Slope of Casting Rate Curves for Various Lots of Powder . . . 6

5: Density Gradient Effect on Part Tolerance . . . . . . . . . . . 6

6: UT Image of Green Tensile Bar Showing Density Gradient . . . . 7

7: SEM Micrograph Showing High Toughness Elongated

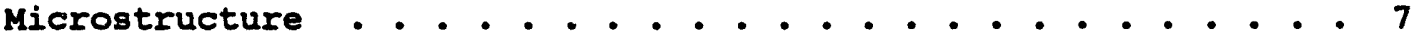

8: Mean Tensile Strengths Using Machining Procedures 1,2,3,4, . . 8

9: Effect of Machining Procedure on the Surface Finish . . . . . 8

10: Liquid Dye Penetrant Fluorescent Microscopy (750X) Revealing

$6 \mu \mathrm{m}$ Wide Grinding Groove and Associated Damage . . . . . . . . 9

11: Intrinsic volume Failure Origin, $25^{\circ} \mathrm{C} 982 \mathrm{MPa}$ Tensile

strength . . . . . . . . . . . . . . . . . . . . . 10

12: Amorphous surface Failure Origin, $25^{\circ} \mathrm{C} 985 \mathrm{MPa}$ Tensile

strength . . . . . . . . . . . . . . . . . . . . 10

13: Inciusion volume Failure origin, $25^{\circ} \mathrm{C} 606 \mathrm{MPa}$ Tensile

strength . . . . . . . . . . . . . . . . . . . . 11

14: Nachining Fracture Origin, $25^{\circ} \mathrm{C} 1234 \mathrm{MPa}$ Tensile

strength . . . . . . . . . . . . . . . . . . . . . 11

15: Cunulative Probability of Failure Plot of stage III Tensile

strength Data . . . . . . . . . . . . . . . . . . . 12

16: Competing Risk Weibull Plot: Intrinsic vs.

Extrinsic Failure Origins . . . . . . . . . . . . . . . . . 13

$x i$ 
17: Cumulative Probability of Failure Plot of Intrinsic

Strength Dala . . . . . . . . . . . . . . . . . 14

18: Intrinsic volume Failure origin, $1370^{\circ} \mathrm{C} 405 \mathrm{MPa}$

Tensile strength . . . . . . . . . . . . . . 15

19: Machining Failure origin, $1370^{\circ} \mathrm{C} 403 \mathrm{MPa}$ Tensile

Strength . . . . . . . . . . . . . . . . 16

20: Tensile stress Rupture Data at $1230^{\circ} \mathrm{C}$ in Air for

Stage II and Stage III specimens . . . . . . . . . . 16

21: Flexure Strength Data from Large Cross Section (MIP)

Specimen . . . . . . . . . . . . . . . . . . 17

1-1: $D_{50}$ Particle Size for As-received and Milled Powder Batches . 28

1-2: Oxygen Level in As-received E03, E05, E10 Ube Powder . . . 29

1-3: Surface Area of As-received E03, E05, E10 Ube Powder .... 30

2-1: Process Flow Chart, W-Series .............. 34

2-2: Baseline Precipitation ................. 34

2-3: Modified Precipitation . . . . . . . . . . . . . 35

2-4: Process Flow Chart, c-Series .............. 35

2-5: Fracture Toughness of Qualification Tiles . . . . . . . 37

2-6: Chart for Particle Size - D50 For C-Series Mill Batches . . . 38

2-7: Casting Rate slope for Lot D, E \& F Mill Batches . . . . . 39

2-8: Fine Scale Iron Silicide Aggregate Failure site . . . . . 41

2-9: Microfocus X-ray Flaw Detection of Inclusions in HIP'ed Rods . 41

2-10: Aging Behavior of Aqueous slurry ..............43

2-11: NMR Aging Behavior of Aqueous slurry . . . . . . . . . 43

2-12: Evaluation of Mold Design by Density Profile Measurement

Using Computed Tomography . . . . . . . . . . . . . . 44

2-13: Nonlinear Casting Rate Under Constant Pressure . . . . . . 45

2-14: Linearized Crsting Rate with Programmed Pressure Schedule . . 46

2-15: Puck Casting $Q$ for slurry ................. 46 
2-16: Regression of Average HIP'ed Runout on Casting Rate . . . . . 47

2-17: RT Strength - Runout Correlation . . . . . . . . . . . 47

2-18: Viscosity vs. Shear Rate for Aqueous Ceramic Suspension . . . 48

2-19: Ultrasonic In-situ Monitoring of Tensile Bar Casting . . . . 50

2-20: UT signal Characteristics as a Function of Casting Time . $^{-} 51$

2-21: Comparison of Casting Rate Prediction from Ultrasonic (B)

and Physical Measurement (A) . . . . . . . . . . . 52

2-22: Injection Molding Melt Flow Velocity by FEM . . . . . . . 53

2-23: Rheological Properties of Injection Molding Compound . . . . 54

2-24: Radiograph Void Volume as Function of Molding Parameters $\cdot$. 55

2-25: Total Flaw Distribution in 240 As-Molded Tensile Bars . . . . 56

2-26: Part Weight Correlated to Radiographic Void volume . . . . 57

2-27: HIP Yield During Stage II Using the HIP Loading

Procedures Without Fixtures . . . . . . . . . . . . 59

2-28: Toughness Moving Average for Stage II . . . . . . . . . . 59

2-29: Lognormal Plot of B Silicon Nitride Grain Thickness

Distributions After BIP at $1840^{\circ} \mathrm{C}$ for Different Soak Times • . 60

2-30: Plot of Aspect Ratio vs. Grain Thickness . . . . . . . . 60

2-31: Fracture Toughness vs. Aspect Frequency ............61

2-32: High Aspect Ratio, High Toughness Microstructure (top)

Compared to Low Toughness Microstructure $(2500 \mathrm{X})$. . . . . 62

3-1: Comparison of Vickers Indent Crack Length Measurements,

IDP v8. SEM ....................... 65

3-2: Metallic Inclusions Detected by MFX Radiography in

Injection Molding Process . . . . . . . . . . . . . 66

3-3: Relative Frequency of Metallic Inclusions Detected in HIP'ed Injection Molded NSF Bars . . . . . . . . . . 67

3-4: Buttonhead Radius Crack (14 mm) Detected by LDP . . . . . . . 68

3-5: Machining Damage in Transition Region of NSF Bar Detected by LDP . . . . . . . . . . . . . . . . 68 
3-6: Void Frequency and Distribution in As-Molded NSF Bars . . . . 69

3-7: NDE/Fractography Inclusion size Coimarison . . . . . . . 69

3-8: Inclusion Size Frequency in HIP'ed NSF Bars From Injection Molding and Pressure Casting Baseline Iterations • 70

3-9: Aging/Agglomeration of Slip Detected by MFX Radiography . . . 71

3-10: Void Frequency in As-Cast Tensile Rods for Casting Iterations V-AA ....................72

3-11: Flaw Statistics from MFX Evaluation of Iteration samples $\cdot$. 73

3-12: Inclusion Population Statistics During Stage II and III Iterations ..................... 74

3-13: MFX Radiography Density Gradient Calibration Si3N4 Step Wedge .. . . . . . . . . . . . . . . 75

3-14: Predicted vs. Actual Tensile strength From NDE Inclusion Data and Fracture Mechanics . . . . . . . . . 76

3-15: Intensity Variations observed in X-ray Beam . . . . . . . . 77

3-16: Air-Entrapment Voids in Cast NSF Bars Detected Radiographically . . . . . . . . . . . . . . 79

3-17: MFX Data Illustrating Density Gradient Control Using Mold Treatment . . . . . . . . . . . . . . . . . 80

3-18: MFX Data Illustrating Powder Lot Variation in Density Gradient .................. 80

3-19: Inclusions Detected by MFX in HIP'ed/Machined Stage III Tensile Rods ..................... 81

3-20: Pores Detected by MFX in HIP'ed/Machined Stage III Tensile Rods .................... 82

3-21: Machining Crack Data Detected by LDP in HIP'ed/Machined Stage III Tensile Rods ................ 83

3-22: A $6 \mu \mathrm{m}$ wide Grinding Groove with 8-35 $\mu \mathrm{m}$ Radial Cracks $(750 \mathrm{X})$...................... 83

3-23: Surface Porosity of Machined Stage III Tensile Rods by IDP . . 84

3-24: Pore Clusters $(13-16 \mu \mathrm{m})$ on Machined Surface of Stage III Tensile Rods . . . . . . . . . . . . . . . . . 84 
3-25: Grinding Grooves ( $5 \mu \mathrm{m}$ wide) Detected by LDP . . . . . . . 85

3-26: Ultrasonic C-scan of NSF Machined Surface Indicating $70 \mu \mathrm{m}$

wide Grinding Grooves . . . . . . . . . . . . . . 85

4-1: Specimen Flow Diagram for Testing and Failure Analysis . . . 87

4-2: Strength vs. Density for Pressure Cast Tensile Rods . . . . . 91

4-3: Failure origin Composed of an Agglomerate at the site of Machining Damage .................. 93

4-4: Fracture Toughness vs. 8 Alpha silicon Nitride . . . . . . 93

4-5: Failure origin of specimen ( $0 \mathrm{f}=898 \mathrm{MPa}$ ) Showing Machining Marks and Agglomerate at the surface . . . . . . 95

4-6: Failure origin of Specimen ( $\sigma \mathrm{f}=879 \mathrm{MPa}$ ) Showing a Severe Circumferential scratch . . . . . . . . . . . . . 95

4-7: Machined Surface and Failure origin of specimen Fractured at $1370^{\circ} \mathrm{C}$ ( $\sigma \mathrm{f}=395 \mathrm{MPa}$ ) Showing $2-20 \mu \mathrm{m}$ Machining Pullouts . . 96

4-8: Failure origin of specimen Fractured at $1230^{\circ} \mathrm{C}$ During stress Rupture showing a Machining Mark and slow Crack Growth . . . 9?

4-9: Failure origin of Specimen Fractured at $1230^{\circ} \mathrm{C}$ During Stress Rupture Showing an Iron/Yttrium Rich Inclusion . . . . . . 97

4-10a:Failure origin (of=596 MPa) Showing a Severe Radial Grinding Mark . . . . . . . . . . . . . . 100

4-10b:Failure origin (of=899 MPa) Showing a $5 \mu \mathrm{m}$ wide Machining Mark .................. . 101

4-10c:Failure Origin (of =889 MPa) Showing a $5 \mu \mathrm{m}$ wide Machining Groove and Sub-surface Thumbnail Shaped Flaw . . 101

4-11: Failure origin ( $\sigma \mathrm{f}=787 \mathrm{MPa})$ showing an Inclusion Containing $\mathrm{Fe}, \mathrm{Cr}, \mathrm{Ni}$. . . . . . . . . . . . . . 102

4-12: Strength-flaw Correlation for Machining Damage Failure origins . . . . . . . . . . . . . . 102

4-13: Mean Tensile strengths From Stage II Iterations . . . . . . 104

4-14: Weibull Plot Assuming an Unimodal Strength Distribution . . 106

4-15: Weibull Plot Using Competing Risk for Surface and volume Failure................... 107 
4-16a:Failure Origin (of $=685 \mathrm{MPa}$ ) Showing Atypical Machining

Damage .................... 107

4-16b:Failure Origin ( $\sigma f=921 \mathrm{MPa}$ ) Showing an Amorphous Region $\cdot 108$

4-16c:Failure Origin ( $\sigma f=944 \mathrm{MPa}$ ) Showing Normal Machining Damage 108

4-16d:Failure origin ( $\sigma f=1040 \mathrm{MPa}$ ) Showing a volume Defect

Containing Microvoids . . . . . . . . . . . . 109

4-17: Competing Risk Weibull Plot for Three Surface Flaw Types . . 109

4-18: Mean Room Temperature Tensile Strength as a Function of Heat

Treatment condition ................ 110

4-19: Mean $1370^{\circ} \mathrm{C}$ Tensile Strength as a Function of Heat Treatment

Condition ...................... 111

4-20: X-ray Diffraction Residual Stress Results . . . . . . . 113

4-21: Typical Load vs. Bending Curves for Strain Gaged Tensile

specimens . . . . . . . . . . . . . . . . 114

4-22: Bending at Failure vs. Tensile strength . . . . . . . . 114

5-1: Tensile Strength Data by Flaw Type . . . . . . . . . 116 


\begin{abstract}
DEVELOPMENT OF IMPROVED PROCESSING AND EVALUATION METHODS FOR HIGH RELIABILITY STRUCTURAL CERAMICS FOR ADVANCED BEAT ENGINE APPLICATIONS, PBASE I
\end{abstract}

Vimal K. Pujari, Dennis M. Tracey, Michael R. Foley, Norman I. Paille, Paul J. Pelletier, Lenny C. Sales, Craig A. Willkens and Russell I. Yeckley

ABSTRACT

This report documents the accomplishments of an extensive four year DOE funded Processing for Reliability R\&D program conducted at Saint-Gobain/Norton Industrial Ceramic Corporation's Northboro Researeh and Development Center (NRDC). The research program had as goals the development and demonstration of significant improvements in processing methods, process controls and nondestructive evaluation directed towards the production of high reliability silicon nitride components for advanced heat engine application. A silicon nitride - 4 w/o yttria composition was used and densification was performed by glass encapsulation hot isostatic pressing (BIP'ing). The material developed in the program has been designated NCX-5102.

The program focused on near-net shape forming the buttonhead tensile bar geometry which allowed a direct means of monitoring product quality and establishing a comprehensive tensile strength database. Extensive NDE and fractography data were generated to establish the nature of failure precursors and their relationships with process parameters. The aqueous based colloidal consolidation process was optimized relative to stringent mechanical property targets. The process includes an innovative closed loop system of uninterrupted colloidal processing, from milling to shape forming, which effectively circumvents traditional sources of contamination. The process demonstration at the end of the program provided over three hundred samples and a corresponding mechanical properties database which suggests that NCX-5102 follows a three parameter Weibull strength distribution with a threshold tensile strength of $665 \mathrm{MPa}$ and a mean tensile strength of over $1 \mathrm{GPa}$. 


\section{INTRODUCTION}

This report discusses highlights of a silicon nitride processing methodology that has been developed in the course of a major four year DOE funded program in processing for reliability. The program focused on the attainment of high strength and reliability through the identification and subsequent control of strength degrading flaw populations. The material produced by the resultant process has been designated $\mathrm{NCX}-5102$ and consists of a silicon nitride -48 yttria composition which is densified by use of glass encapsulation uIP'ing. An account is provided of developmental efforts which have resulted in a colloidal processing methodology for the production of high strength, high reliability silicon nitride components for advanced heat engine application at temperatures to $1370^{\circ} \mathrm{C}$. The effort has concentrated on the near net shape fabrication of large aspect ratio, axisymetric buttonhead tensile bars. This geometry served as a challenging forming requirement, sufficient for development of a general shape forming capability and it also provided a direct means to assess tensile strength levels attributable to the process.

The program was conducted in three stages, namely: 1) baseline process definition, 2 ) process optimization and 3) large scale production demonstration. In stage $I$, two distinct processing routes injection molding and colloidal consolidation (CC) - were evaluated and the CC route was found to offer the best potential for quality and costeffective manufacturing. In Stage II, the CC process was optimized relative to stringent mechanical property targets. The overall program goal was to achieve a mean room temperature tensile strength of $900 \mathrm{MPa}$ with a (2-parameter) Weibull modulus of 20 , a mean $1370^{\circ} \mathrm{C}$ tensile strength of $500 \mathrm{MPa}$ and a mean $1230^{\circ} \mathrm{C}, 350 \mathrm{MPa}$ tensile stress rupture life of 100 hours.

A "closed loop" aqueous based powder beneficiation - colloidal consolidation methodology was the basis of the green ceramic production strategy. Large scale batches $(30 \mathrm{~kg})$ of silicon nitride - 48 yttria powder were milled in water and the resulting slurry was used to cast as many as 200 tensile rods. Ube powder which is derived from a diimide process was used as starting silicon nitride powder. The processing was conducted within a class 10000 clean room. More than thirty full scale batches were processed which allowed the development of SPC charts for several chemical and physical properties of the powder and slurry for monitoring of variations in raw material as well as the milling, filtration and concentration operations. To ensure process repeatability, a comprehensive standard operating procedure (SOP) was established for each subtask of each unit operation.

The casting operation was optimized using a custom designed multistation casting rig. The apparatus was computer controlled according to a pressure profile established to maintain a uniform casting rate. This results in a well controlled green microstructure having minimal density variation.

The process optimization strategy involved a series of process iterations. The tensile specimens produced in each iteration were tested to determine the tensile strength distribution representative of 
the iteration. Each iteration served as an experiment in a test matrix which was designed with the critical process parameters as variables. Extensive NDE and fractography data were generated to establish the nature of failure precursors for guidance on the process improvements necessary for stre 1gth and reliability enhancement in subsequent iterations. The resulting data for the set of experiments were used to define the optimum, total process, control variable levels.

The process control research employed experimental design methods to establish cause-effect relationships involving key process variables identified during stage I. Control parameters were defined during the course of this iterative experimentation and statistical process control (SPC) methods were implemented to assure process reproducibility. Notable examples of innovation include a method for control of the colloidal consolidation process (pressure casting operation) for formation of a uniform density green microstructure, BIP cycle definition which assures a high toughness acicular grain microstructure at greater than 99.58 theoretical density, and a machining methodology for the cylindrical tensile bars which maintained control of strength impairing surface damage.

Several NDE techniques were employed, including microfocus radiography (MFX), ultrasonics (UT), computer tomography (CT), fluorescent liquid dye penetrant (LDP) and nuclear magnetic resonance (NMR). The techniques were adapted for application to various steps of the process. The detection capabilities of each technique were determined quantitatively with appropriate reference standards which were prepared at Northboro R\&D Center. The NDE methods were customized during stage II to provide novel characterization tools found necessary in the effort to establish cause-effect relationships. NDE played a crucial role as well in the areas of process monitoring and in-process flaw detection.

The cylindrical buttonhead tensile specimens were machined to the ORNL design with a gage diameter of $6.0 \pm 0.1 \mathrm{~mm}$. All specimens for the main body of strength data were machined using a specially designed sop developed for this geometry. Two comprehensive studies were pursued to establish optimum machining practice. The first involved a machine shop qualification which established specifications for control of buttonhead failures. The second involved specifications for control of machining damage in the gage length which influences tensile strength of NSF bars. optical fractography and scanning electron microscopy were performed on all specimens. Low and high magnification micrographs were taken of each fracture surface and flaw origin. Energy dispersant spectroscopy was performed at each origin to determine if any impurities were present. The location, type and size of each flaw were noted, measured and tabulated for database evaluation.

The stage III demonstration provided over three hundred samples and a corresponding mechanical properties database. Fabricability of large cross section components was assessed by forming $50 \mathrm{~mm}$ diameter samples. Mechanical properties of these samples were established by testing specimens machined from locations across the samples. 
POWDER PROCESSING

The aqueous closed loop process is compared with the conventional alcohol milling approach in Figure 1. Various physical and chemical characterization techniques were employed both for the raw material and the milled powder. Although $x$-ray diffraction of the starting ube powder found very little variation in $\alpha$-phase from lot to lot, measurable variations in surface area (shown in Figure 2), particle size and oxygen content were found. In most cases, milling conditions were adjusted to bring the powder properties within the specified control limits. However, the fifth lot of powder, "Lot $E$ ", in spite of meeting all physical property specifications, showed very different colloidal casting behavior. Further surface chemical examinations (courtesy Dr. s. Malghan, NIST) suggested significant shift in the iso-electric point (IEP) for the Lot $E$ powder (as compared to Lot D) as shown in Figure 3 . other lots of powder showed similar behavior to that of Lot $D$. Various surface treatments have been developed to control the IEP and hence the casting behavior. IEP was also included as a process control parameter.

\section{ALCOHOL ROUTE}

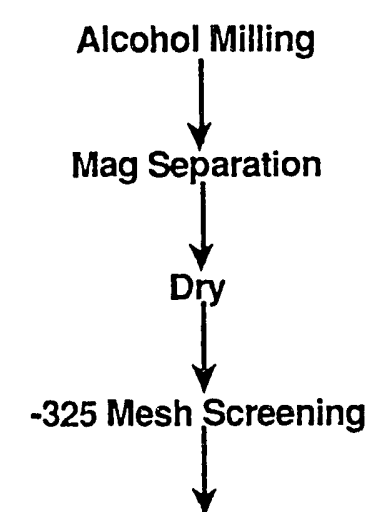

Disperse in Water

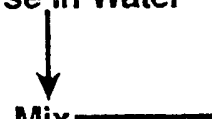

$$
\text { Mix }
$$
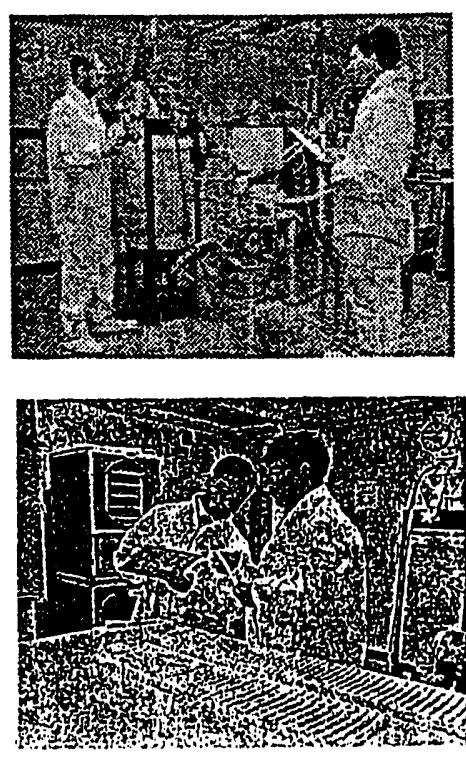

Cast Body

\title{
AQUEOUS ROUTE
}

\author{
Aqueous Milling<smiles>C[Te]</smiles> \\ Mag Separation<smiles>[Y]C</smiles> \\ Filtration $(5 \mu \mathrm{m})$
}

Figure 1: Comparison of Aqueous and Alcohol Based Powder Processing Methods 


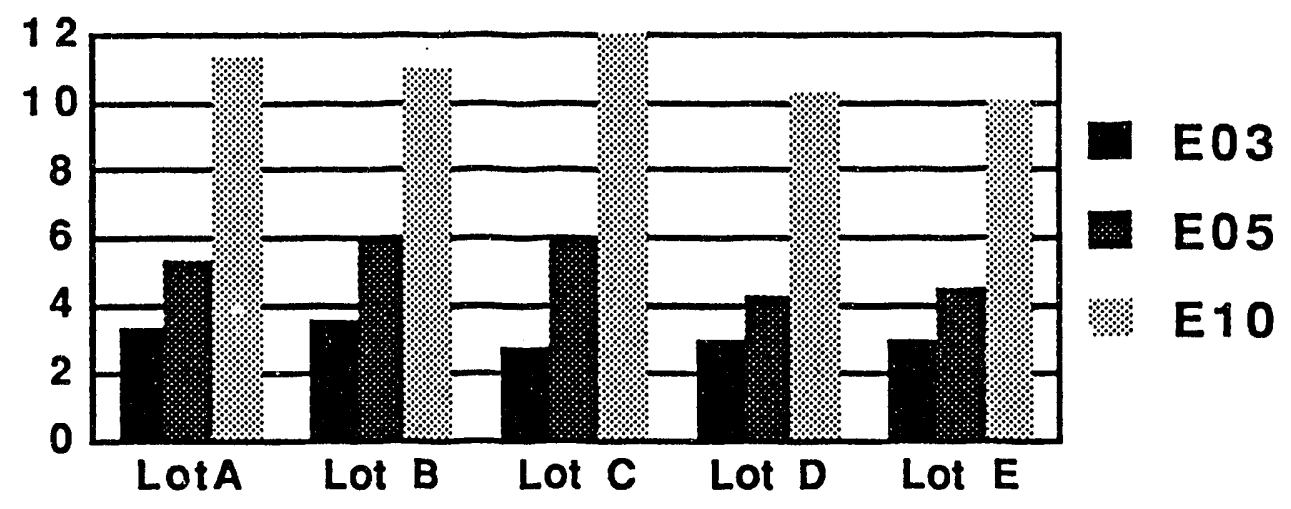

Figure 2: Surface Area for Various Grades and Lots of UBE Silicon Nitride Powder

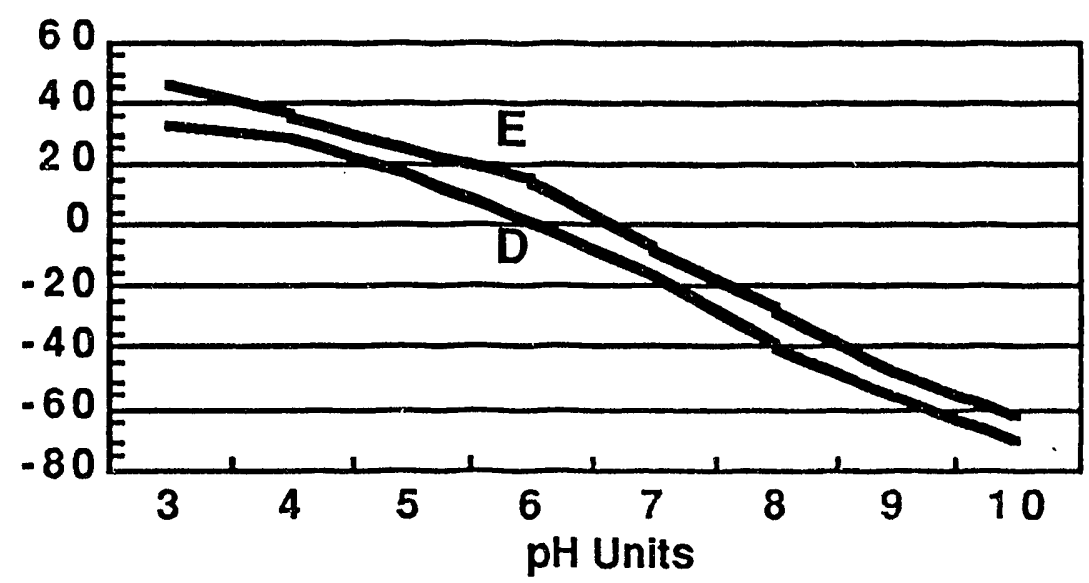

Figure 3: Zeta Potential vs. pH for SisN4 Lots D and E

\section{SLURRY DEVELOPMENT AND CHARACTERIZATION}

A comprehensive slurry characterization methodology was developed using viscosity, $\mathrm{pH}$, and centrifuge packing density measurements in combination with the NMR (Nuclear Magnetic Resonance) spin-lattice parameter (T1). Slurry characterization was further refined to predict casting behavior. A "Casting Rate slope" parameter was defined and developed as a process control measure. The procedure is briefly described as follows: a fixed amount of slurry is cast at a constant pressure on an established porous material. Cast thickness is monitored at fixed time intervals. Thickness squared is plotted as a function of casting time and the slope of the line is defined as the "Casting Rate slope" (CRS). An Upper Control limit (UCL) for CRS is defined as shown in Figure 4. Slurries having CRS values above UCL are rejected, as high CRS correlates with unacceptable density gradient levels in the cast body as described in the next section. 

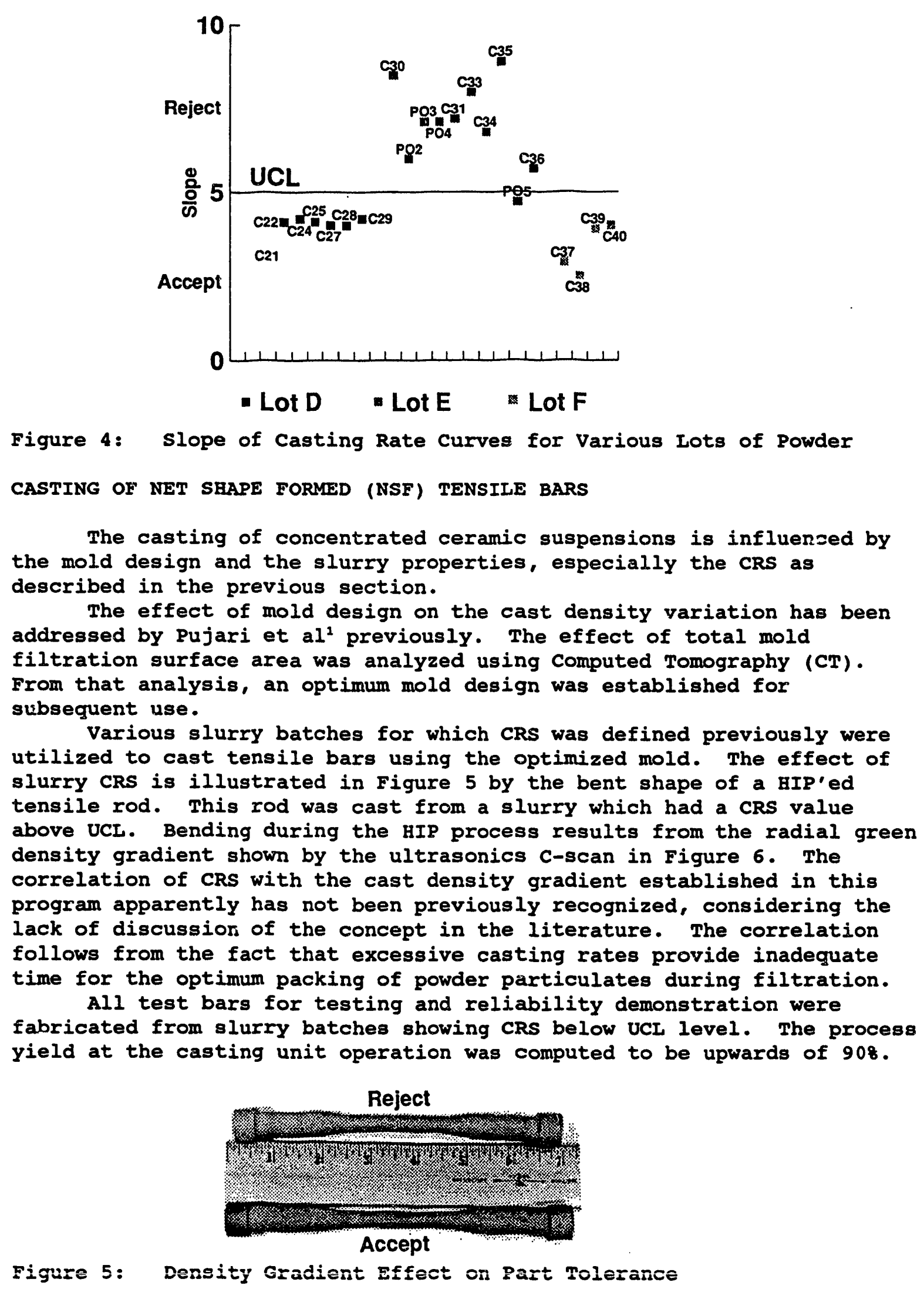


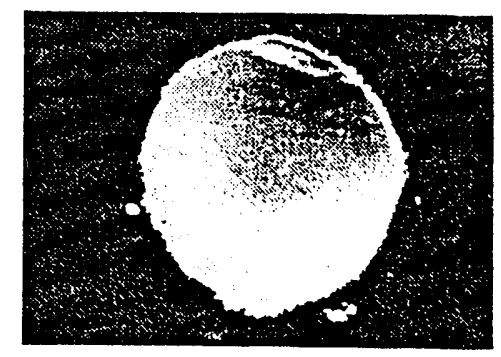

Figure 6: UT Image of Green Tensile Bar Showing Density Gradient HOT ISOSTATIC PRESSING

The net shape formed (NSF) bars were hot isostatically pressed (HIP'ed) using ASEA glass encapsulation technology. The HIP process was optimized using pressure, time and temperature parameters to assure full densification and development of an elongated microstructure (Figure 7) for desired fracture toughness (KIC). Control limits for density and fracture toughness were defined as $>99.58 \mathrm{TD}$ and $5.8<\mathrm{KIC}<6.2 \mathrm{MPa} \sqrt{\mathrm{m}}$, respectively. It was imperative to reproduce KIC within specified control limits in order to examine the process improvement due to the flaw reduction, a key objective of this program. It was, however, found that the HIP process contributed to the process variability by nature of its being a batch process. Better understanding of the thermal profiles during the HIP cycle and/or incorporation of a continuous sintering cycle will further enhance the reliability of this improved process. A limited effort has been launched towards understanding the temperature gradients during sintering in general and in the HIP cycle in particular.

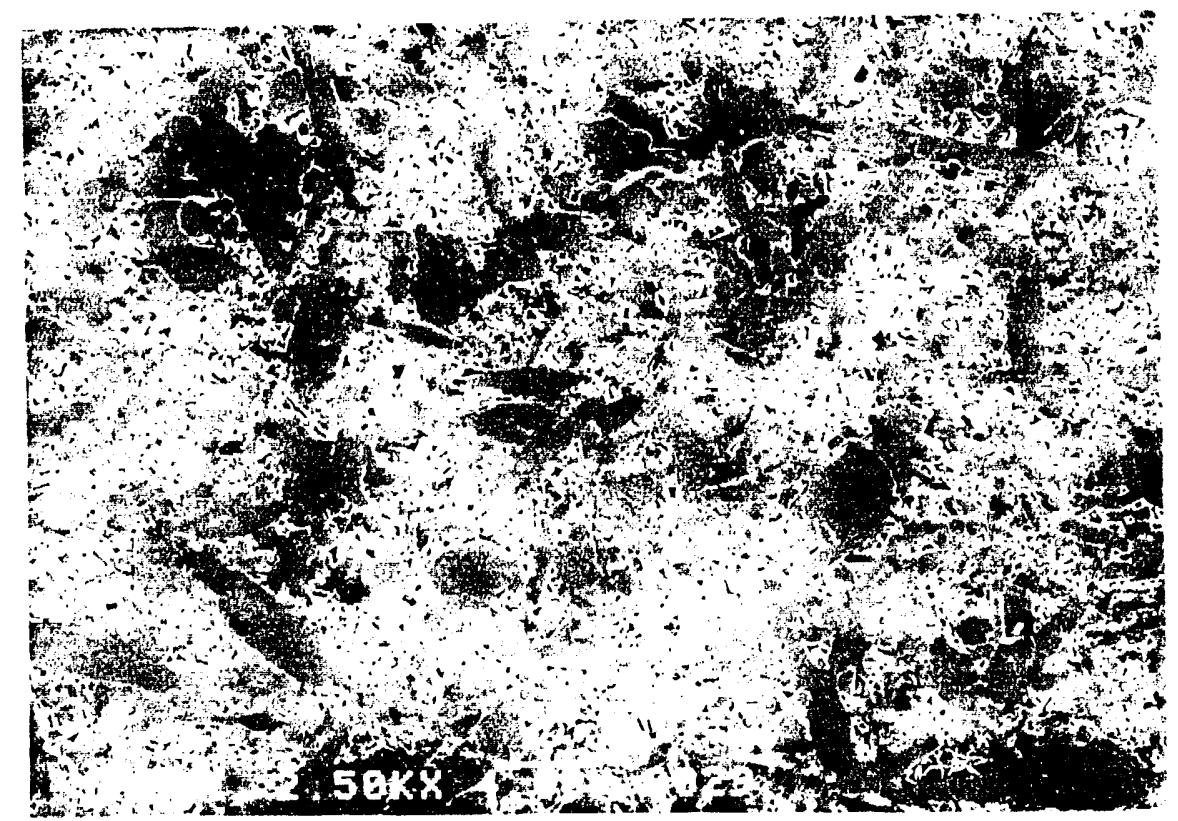

Figure 7: SEM Micrograph Showing High Toughness Elongated Microstructure 
MACHINING PROCESS OPTIMIZATION

Four machining procedures were evaluated for their influence on tensile strength. A total of 72 tensile specimens from 3 HIP runs were machined and tested. Consistent with the focus on machining damage effects, the specimens were not given heat treatments for the purpose of this study. To properly evaluate the procedures, only those specimens that failed from surface flaws within the gage length were considered (64 of 72).

Customary practice expresses surface finish specification in terms of the average roughness Ra. Early stage II work established the need to revise the $R a$ specification from $16 \mu$ in $(0.4 \mu \mathrm{m})$ to $10 \mu$ in at the buttonhead radius and to $8 \mu \mathrm{in}$ along the gage length. The current study maintained these Ra specifications but considered different rough grinding procedures including intermediate grinding steps, diamond wheel type and depths of cut. On the basis of the grinding parameters employed, the machining procedures would be ranked according to operational precision from \#1 to \#4, with \#4 being the most precise.

In spite of the major differences in operational precision, the resultant tensile strength data showed no statistically significant dependence on procedure, Figure 8, even though Ry (maximum peak to valley) Talysurf surface finish values were significantly higher for procedures \#1 and \#2 when compared to procedures, \#3 and \#4, Figure 9.

The mean strengths found in this study were consistent with the $900 \mathrm{MPa}$ room temperature target, given the absence of strength enhancing heat treatment. Therefore the primary consideration in choosing a machining procedure for stage III was attaining a minimum variability of strength and high Weibull modulus. Since procedure \#l had the least variability, it was chosen as soP for stage III demonstration.

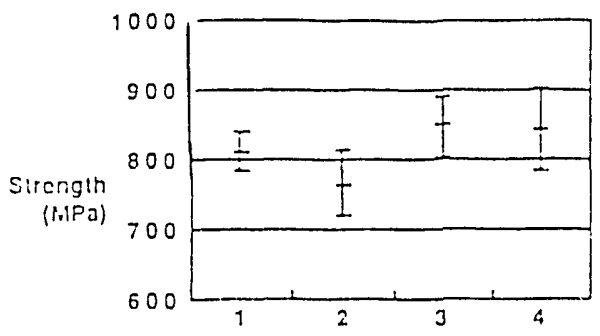

Figure 8: Mean Tensile Strengths Using Machining Procedures 1,2,3,4

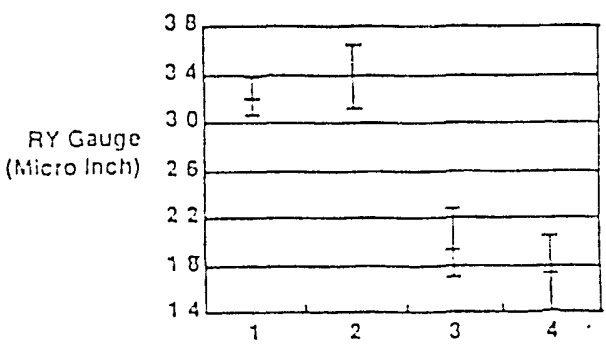

Figure 9: Effect of Machining Procedure on the Surface Finish 


\section{TENSILE STRENGTE DATABASE}

Over the course of the four year program, hundreds of tensile rods were fabricated and tested. The final stage III demonstration involved over 300 samples which were tested to establish the room temperature fast fracture tersile strength distribution characteristic of the optimized process. In addition, more than 25 elevated temperature fast frácture $\left(1370^{\circ} \mathrm{C}\right)$ and stress rupture $\left(1230^{\circ} \mathrm{C}\right)$ tensile tests were completed as discussed below.

\section{ROOM TEMPERATURE TENSILE TEST DATA}

The tensile test database from the stage III demonstration set include's fractography results on location, type and size of the failure origins obtained using optical and scanning electron microscopy. The majority oi the room temperature specimens had failure origins at the surface within the uniformly stressed $6 \mathrm{~mm}$ diameter gage length. Two types of surface failure origins were identified; namely, machining flaws and amorphous areas that developed during the post-machining treatment step. Also, two distinct types of volume flaws were observed. One was a microstructural flaw characterized by a zone of microporosity. The other involved inclusions from contamination subsequently traced to a piece of processing equipment. The fractography results are summarized in Tablo 1 according to failure origın type. A typical machining flaw which includes a grinding groove and associated radial cracks as depicted by the liquid dye penetrant technique is shown in Figure 10. Fractography examples of the four flaw types are shown in Figures 11-14.

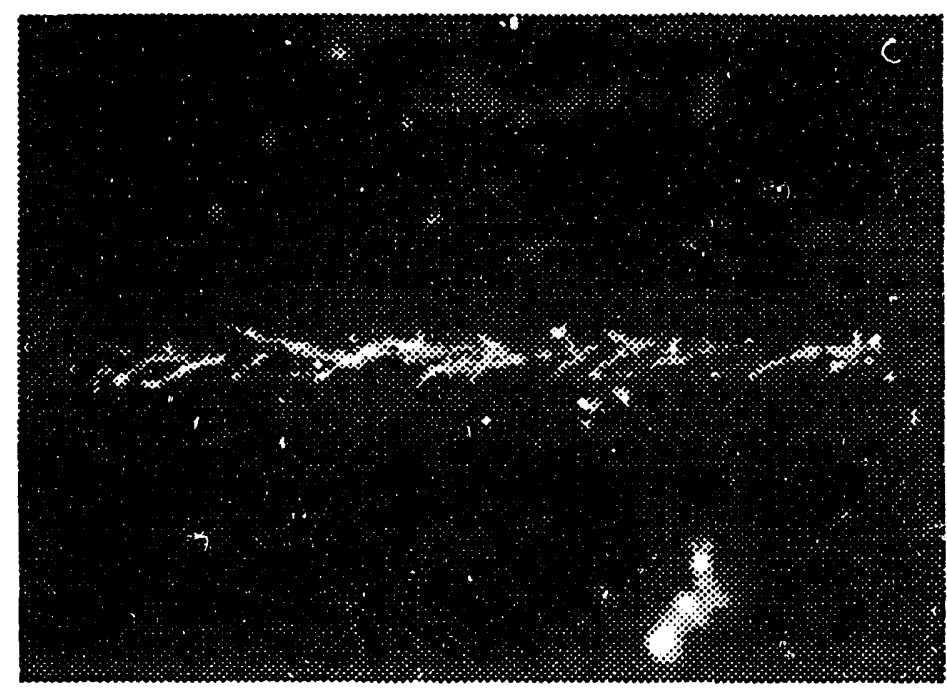

Figure 10: Liquid Dye Penetrant Fluoresce: Microscopy (750X) Revealing $6 \mu \mathrm{m}$ Wide Grinding Groove and Associated Damage 

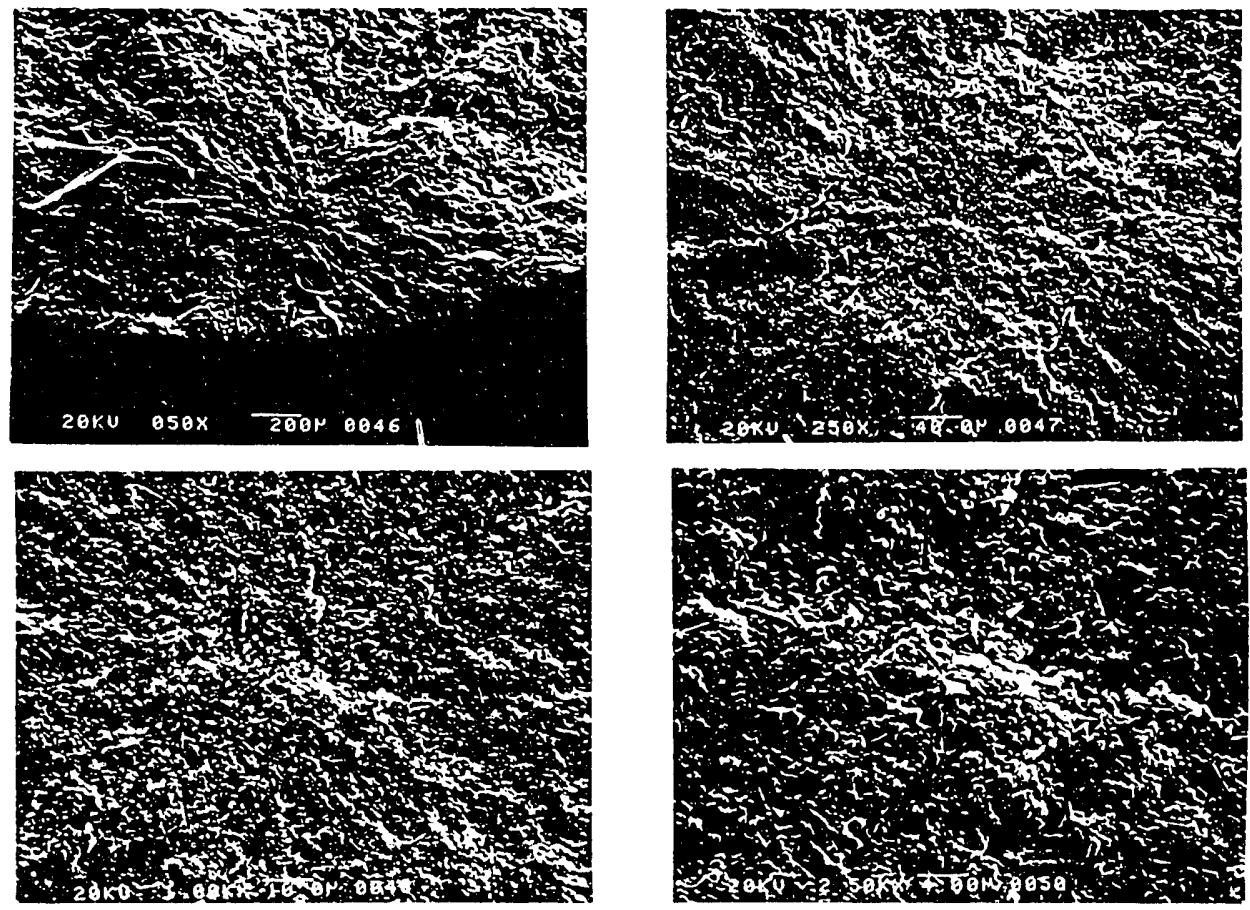

Figure 11: Intrinsic Volume Failure origin, $25^{\circ} \mathrm{C} 982 \mathrm{MPa}$ Tensile strength
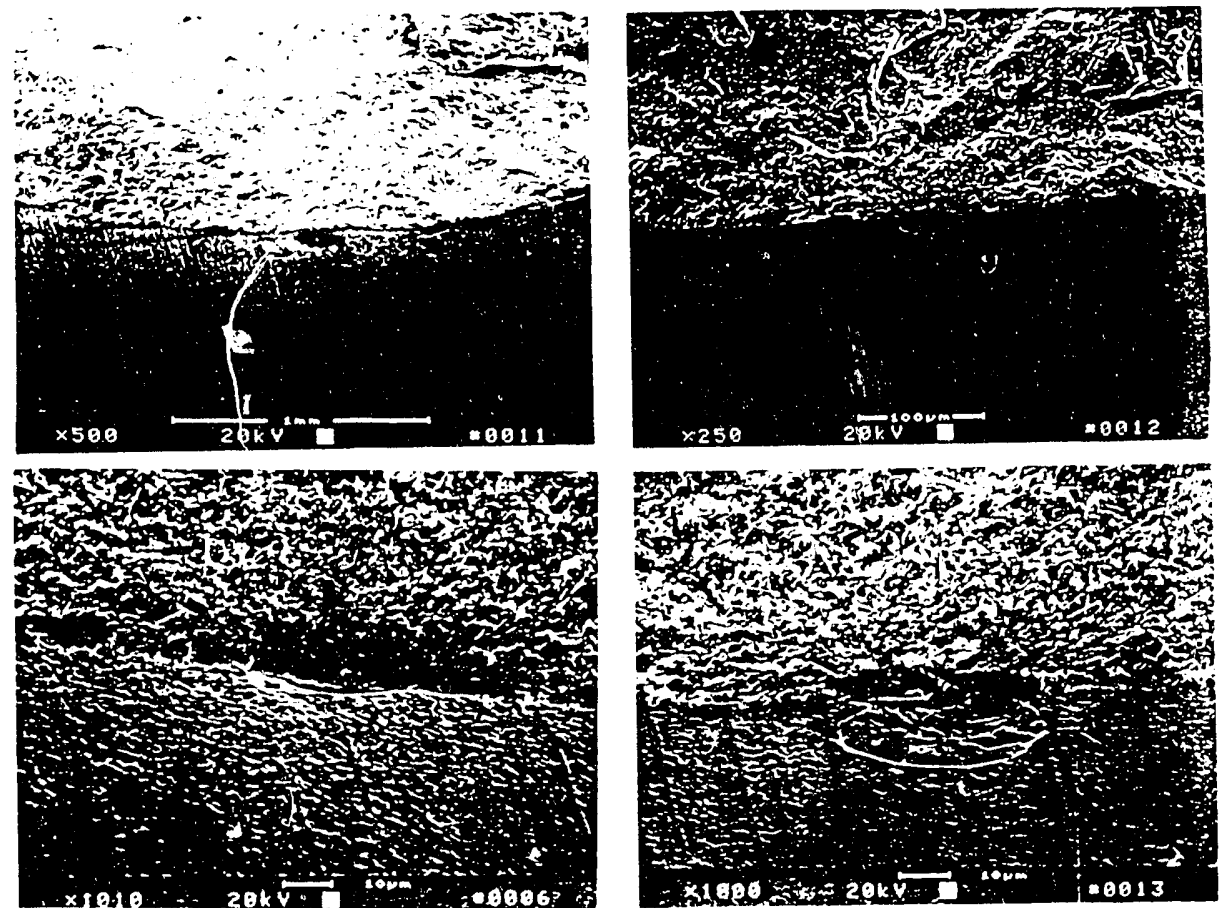

Figure 12: Amornhous surface Failure origin, $25^{\circ} \mathrm{C} 985 \mathrm{MPa}$ Tensile strength 

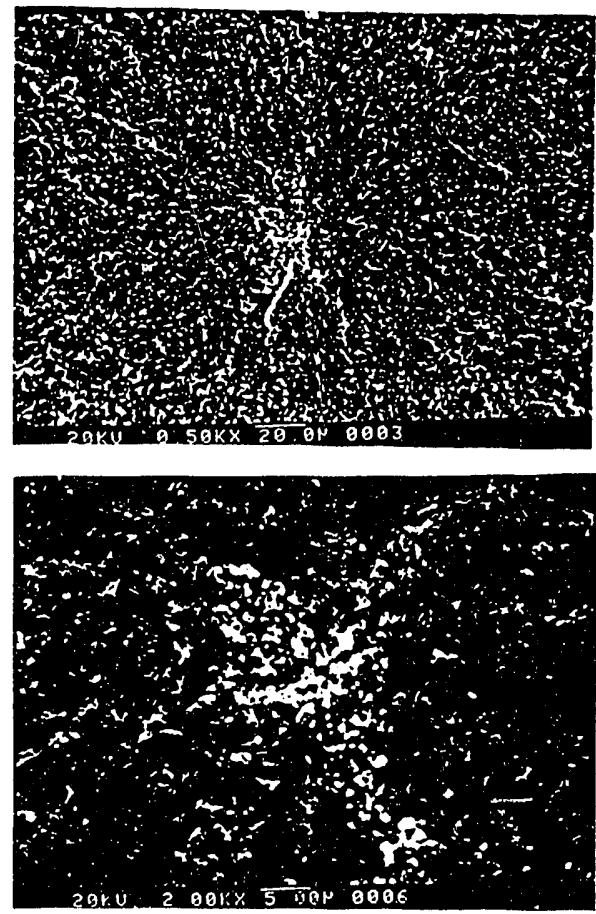

Figure 13: Inclusion Volume Failure origin, $25^{\circ} \mathrm{C} 606 \mathrm{MPa}$ Tensile strength
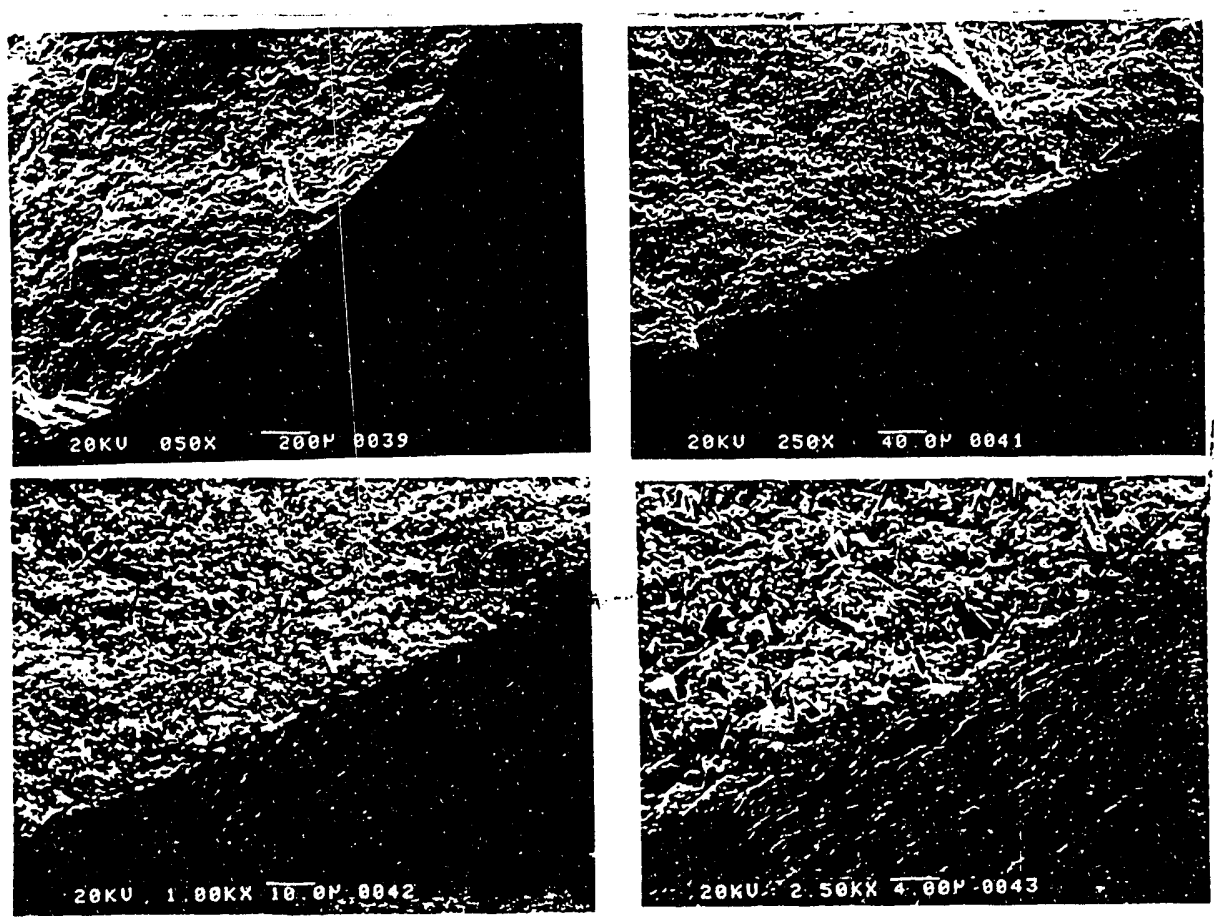

Figure 14: Machining Fracture Origin, $25^{\circ} \mathrm{C} 1234 \mathrm{MPa}$ Tensile strength 
Table 1: Stage III Fractography $\left(25^{\circ} \mathrm{C}\right)$ Summary

\begin{tabular}{||l|l|l|c|}
\hline Category & Type & Location & $\#$ \\
\hline \multirow{3}{*}{ Intrinsic } & Microporous & Volume & 55 \\
\cline { 2 - 4 } & Amorphous & Surface & 107 \\
\cline { 2 - 4 } & Unknown & & 8 \\
\hline \multirow{3}{*}{ Extrinsic } & Inclusion & Volume & 16 \\
\cline { 2 - 4 } & Machining & Surface & 134 \\
\hline
\end{tabular}

In the statistical analysis discussed below, the surface amorphous flaws and the volume microstructural flaws were considered to comprise a set of intrinsic flaws, since they randomly occur within the standard processing cycle. On the other hand, the machining damage and inclusion flaws were classified as being extrinsic flaws, since they are subject to control by means unrelated to the silicon nitride process per se.

The database includes a total of 320 valid tensile tests. There were an additional 35 specimens which failed in the non-uniformly stressed regions of the specimen which have been excluded from this summary. The 320 strength data are presented in a cumulative probability of failure plot using standard Weibull coordinates in Figure 15. The data range from 540 to $1237 \mathrm{MPa}$ and have a mean value of 997 MPa. The strength and fractography data are tabulated in Appendix 5. The nonlinear character of the distribution with multiple inflections suggests that a 2-parameter Weibull fit of this data $\left(\sigma_{0}=1038 \mathrm{MPa}\right.$, $m=10.4$ ) would be inappropriate and that the multimodal nature of the data should be represented using competing risk analysis.

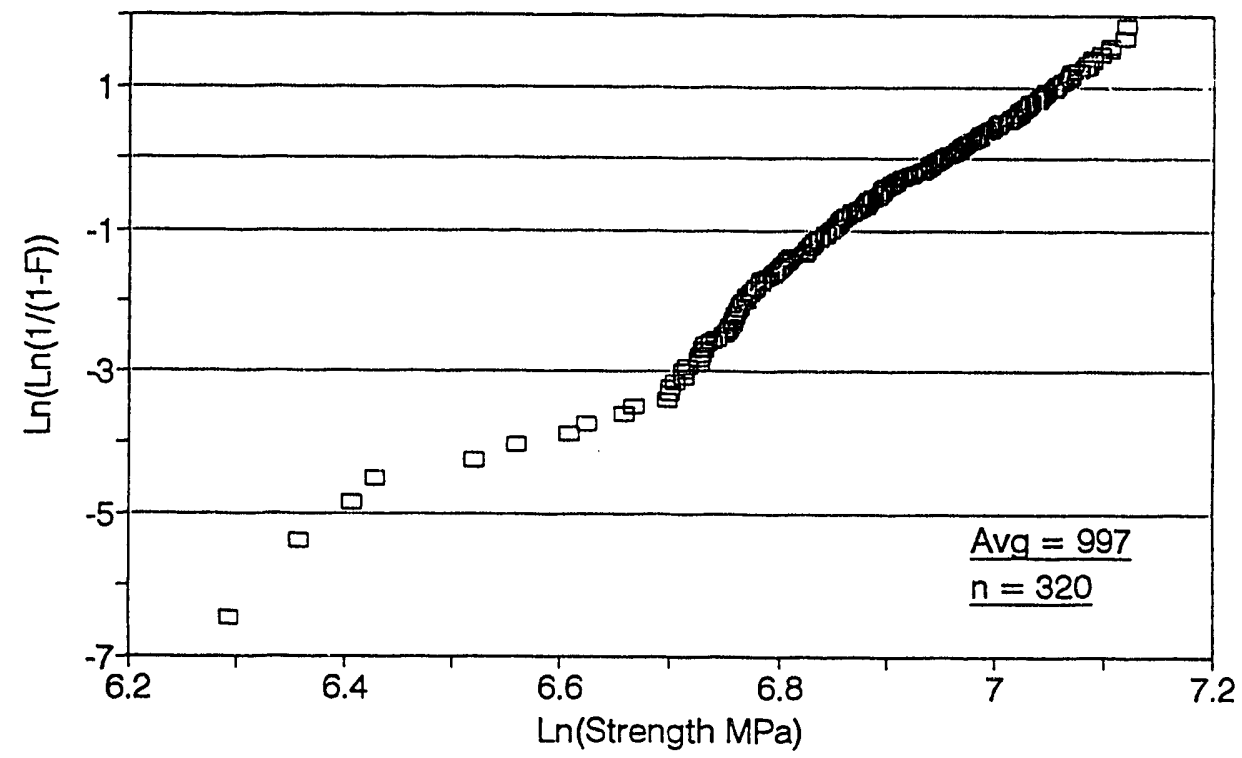

Figure 15: Cumulative Probability of Failure Plot of stage III Tensile Strength Data 
The multimodal analysis was pursued by considering the group of 320 strength data as being composed of two sets, with one set $(n=170)$ representing strengths of the intrinsic defects and the other $(n=150)$ representing strengths of the extrinsic defects, Figure 16. Competing risk analysis reveals that the extrinsic strength data dominate the nonlinear low strength tail of the overall distribution. The plot of their distribution is similar to that of Figure 15. The intrinsic strength data on the other hand has a distinctly different character which is well represented by a 3-parameter Weibull model with a threshold stress of $665 \mathrm{MPa}$, a scale value of $444 \mathrm{MPa}$ and a shape value of 3.92. The intrinsic strength data are plotted in Figure 17 along with this 3-parameter model and also with a 2-parameter fit which clearly is a poor representation of the data. The line to the left of the data represents the program target material having a mean tensile strength of $900 \mathrm{MPa}$ and a 2-parameter Weibull modulus of 20 .

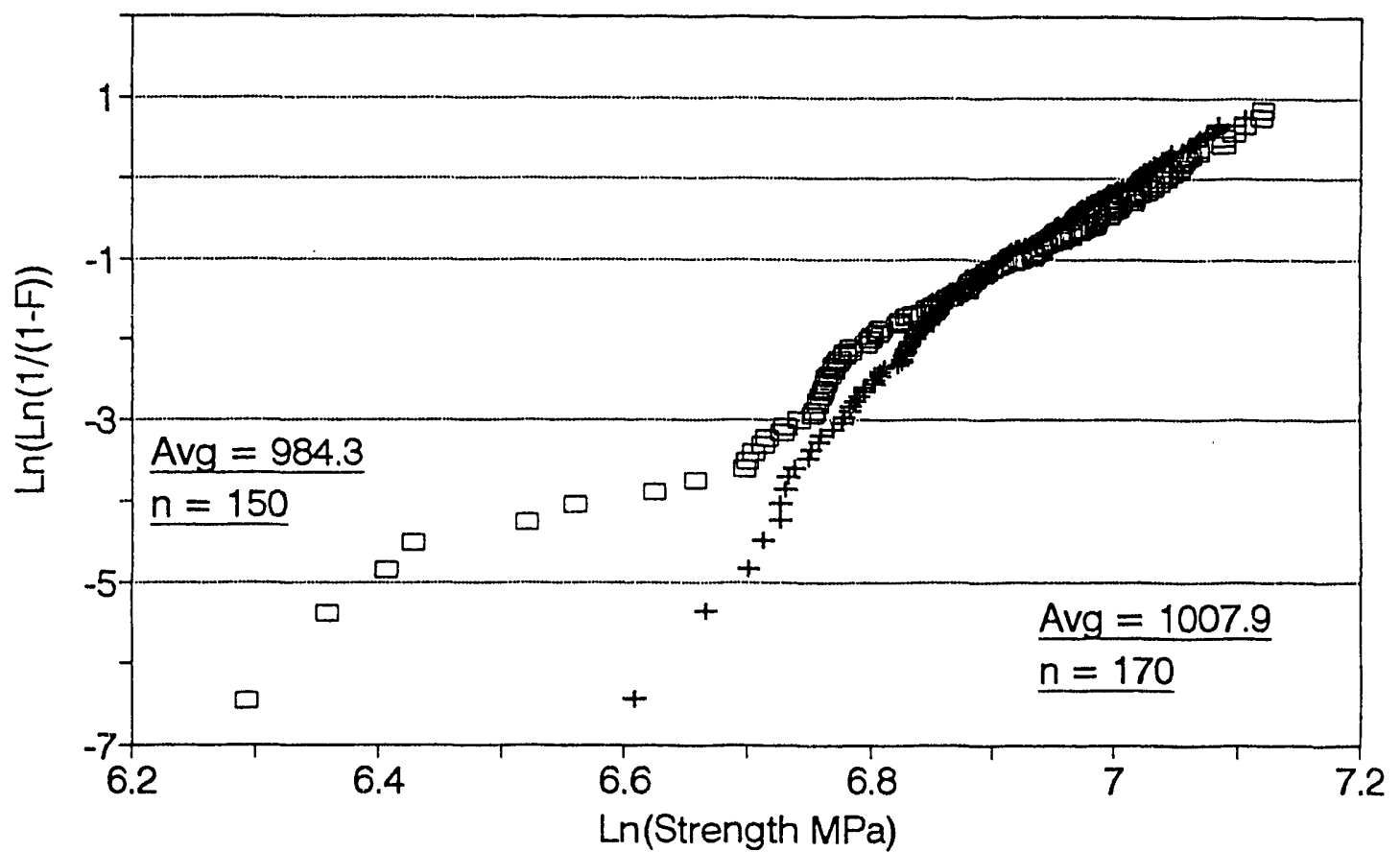

Figure 16: Competing Risk Weibull plot: Intrinsic vs. Extrinsic Failure origins

From a reliability perspective, the important feature of the 3 parameter Weibull distribution is the existence of a threshold stress below which there is zero probability of failure ${ }^{2}$. Physically this corresponds to a processing methodology which has succeeded in truncating the upper tails of the critical flaw size distributions. A threshold at the level indicated clearly has direct impact on design systems used in fielding reliable ceramic components. For instance, NCX-5102 with the 3 parameter distribution of Figure 17 would provide higher reliability at all stress levels when compared to the program target material, as indicated in Table 2 . 
Table 2: Probability of Survival at Given Stresses (MPa)

\begin{tabular}{|l|l|c|c||}
\hline & Target & $\begin{array}{l}\text { Weibull 2 } \\
\text { Parameter }\end{array}$ & $\begin{array}{l}\text { Weibull 3 } \\
\text { Parameter }\end{array}$ \\
\hline \hline R99 & 735 & 766 & 802 \\
\hline R999 & 655 & 639 & 741 \\
\hline R9999 & 583 & 533 & 707 \\
\hline R99999 & 520 & 444 & 689 \\
\hline R999999 & 463 & 370 & 678 \\
\hline
\end{tabular}

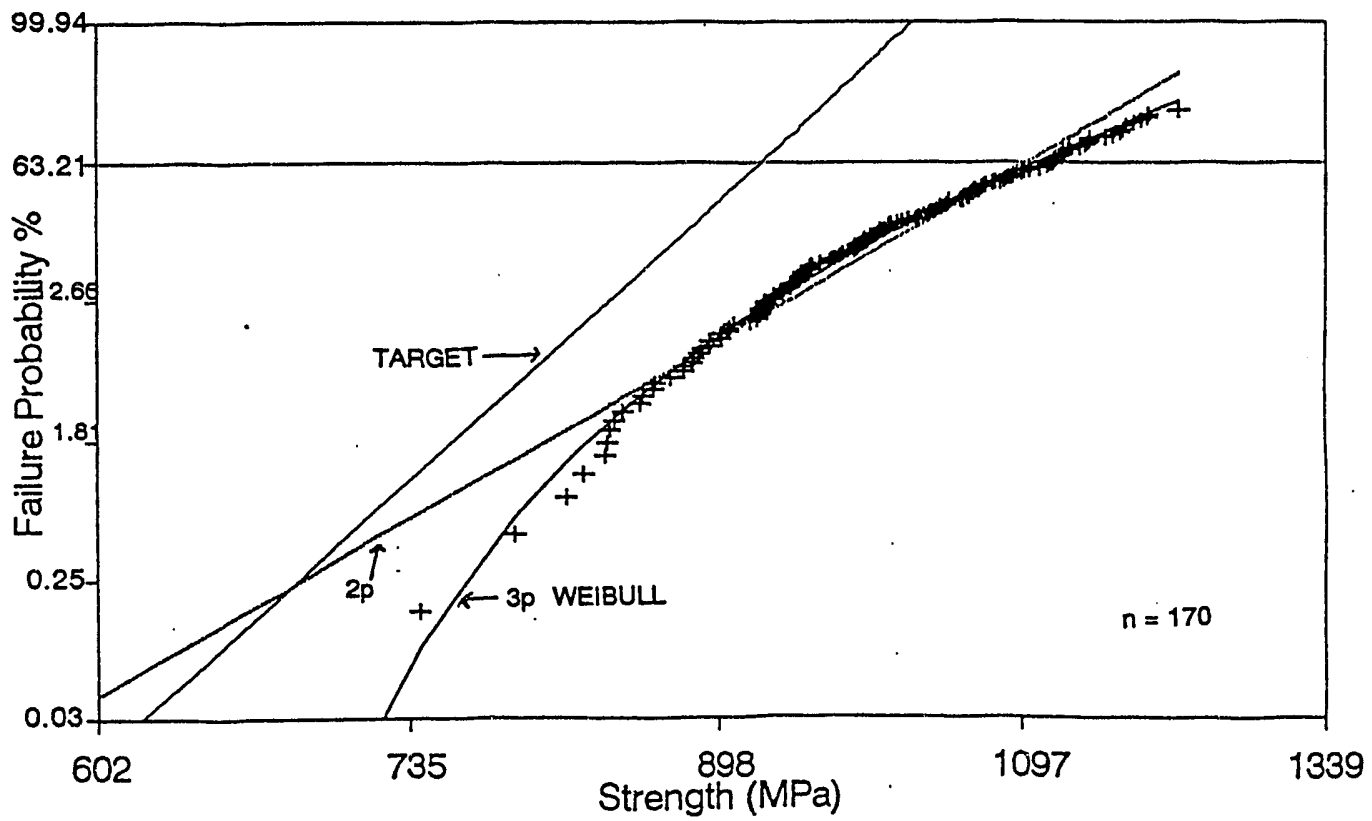

Figure 17: Cumulative Probability of Failure Plot of Intrinsic strength Data

ELEVATED TEMPERATURE TENSILE TEST DATA

The potential of reaching the program's elevated temperature mechanical property targets was demonstrated during stage II using a specialized sop. Fast fracture strengths at $1370^{\circ} \mathrm{C}$ were found to range from 425 to $520 \mathrm{MPa}$ with a mean of $477 \mathrm{MPa}$ in 5 tests. Stress rupture lives at $1230^{\circ} \mathrm{C}$ equaled or exceeded 100 hours at 250,300 and $350 \mathrm{MPa}$ in separate tests, although not all tests at $350 \mathrm{MPa}$ survived 100 hours.

It was found that processing conditions for optimum room temperature properties differed from the above sop. The primary focus in the program was to establish strength and reliability improvements through flaw control using room temperature strength data as the measure of improvement. On this basis, the Stage III optimization focused on 
SOP which favored room temperature characterization of NCX-5102.

Fifteen Stage III samples were tested to fast fracture at $1370^{\circ} \mathrm{C}$. The mean strength was $396 \mathrm{MPa}$, with a range 344-452 MPa. There were 6 volume and 9 surface failures and fractographic examples of each are given in Figures 18 and 19.

Ten Stage III stress rupture tests were conducted at $1230^{\circ} \mathrm{C}$. The target 100 hour lifetime was achieved at $250 \mathrm{MPa}$, but not at higher stress levels. The data from the stage II and Stage III tests are shown in Figure 20 in a plot of applied stress vs. failure time.

Numerous processing steps were optimized to produce superior room temperature properties to demonstrate that flaw control can result in significant advances in the quality of silicon nitride. Additional processing optimization can be expected to provide improved high temperature properties beyond what has been accomplished in this program as was demonstrated by the investigation using the specialized sop cited above.
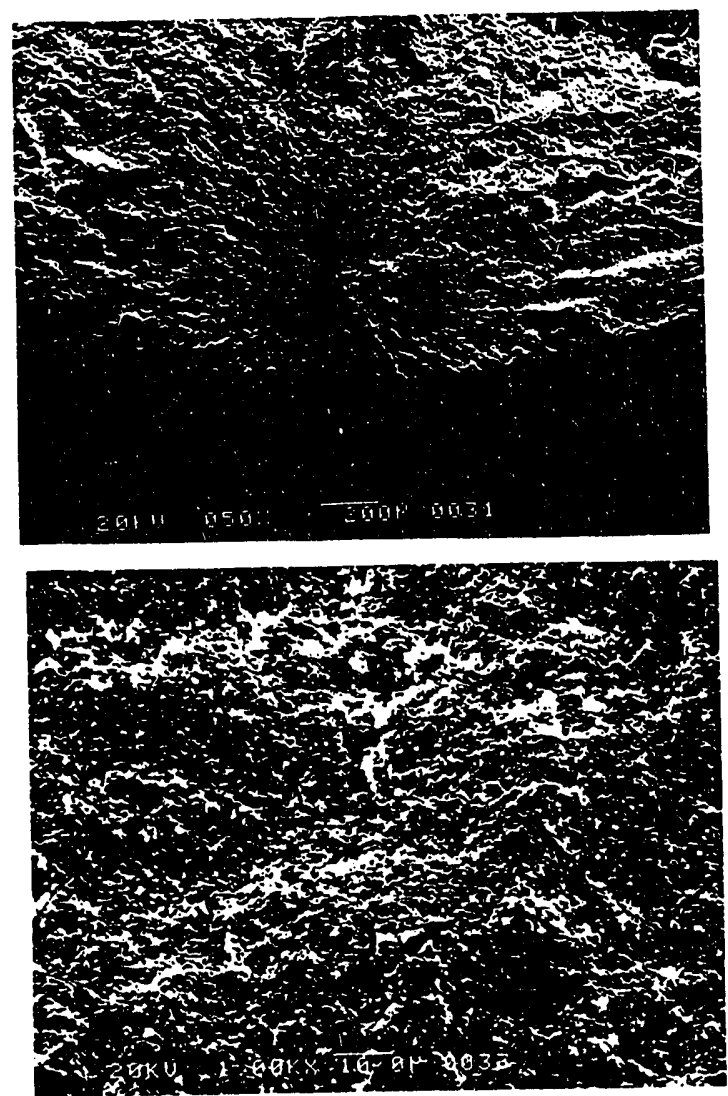
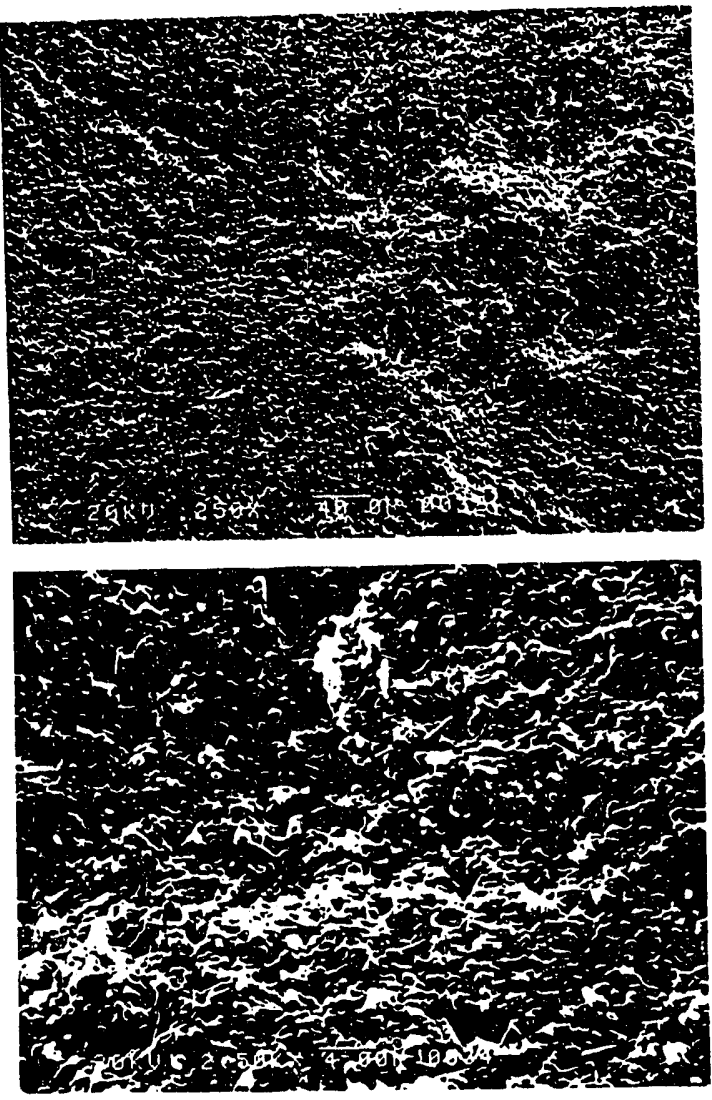

Figure 18: Intrinsic Volume Failure origin, $1370^{\circ} \mathrm{C} 405 \mathrm{MPa}$ Tensile strength 

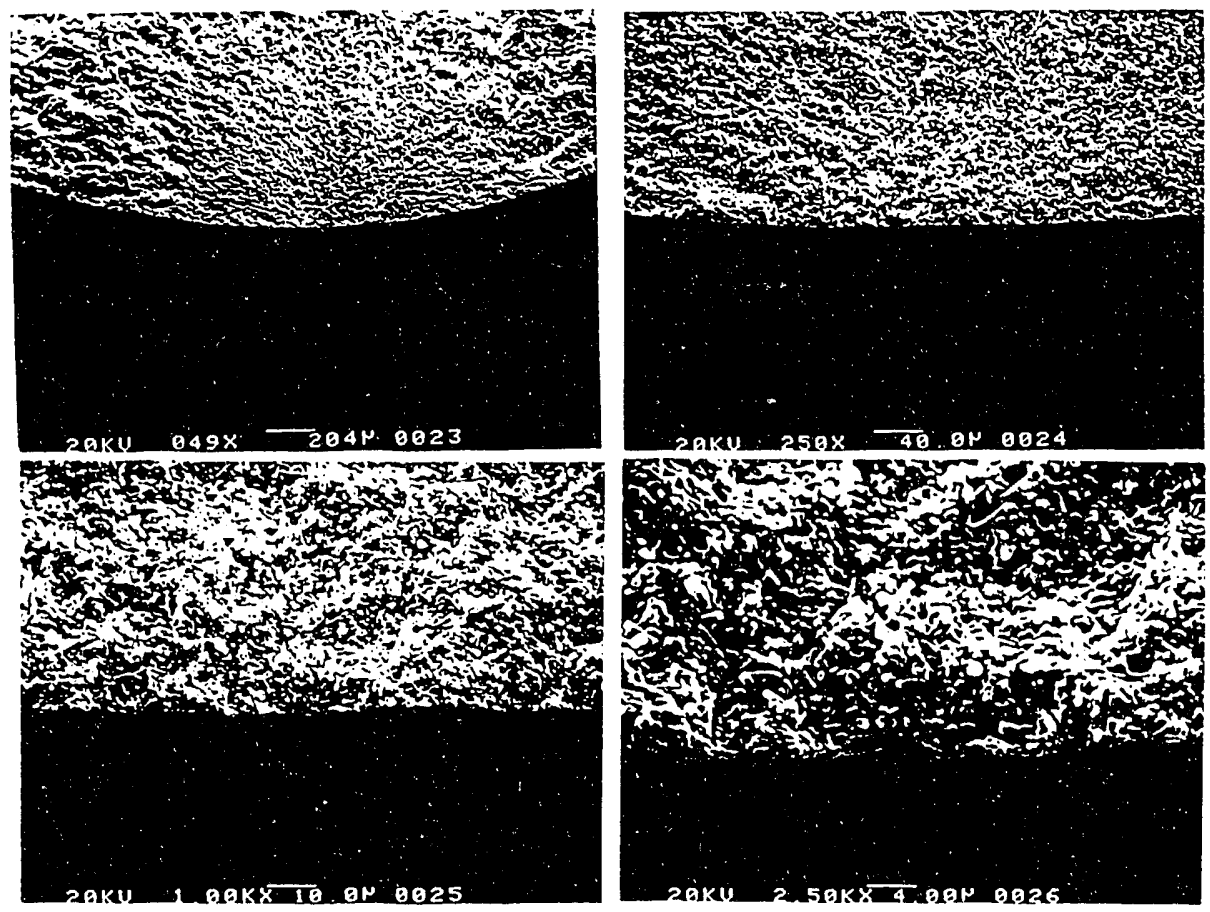

Figure 19: Machining Failure origin, $1370^{\circ} \mathrm{C} 403 \mathrm{MPa}$ Tensile Strength

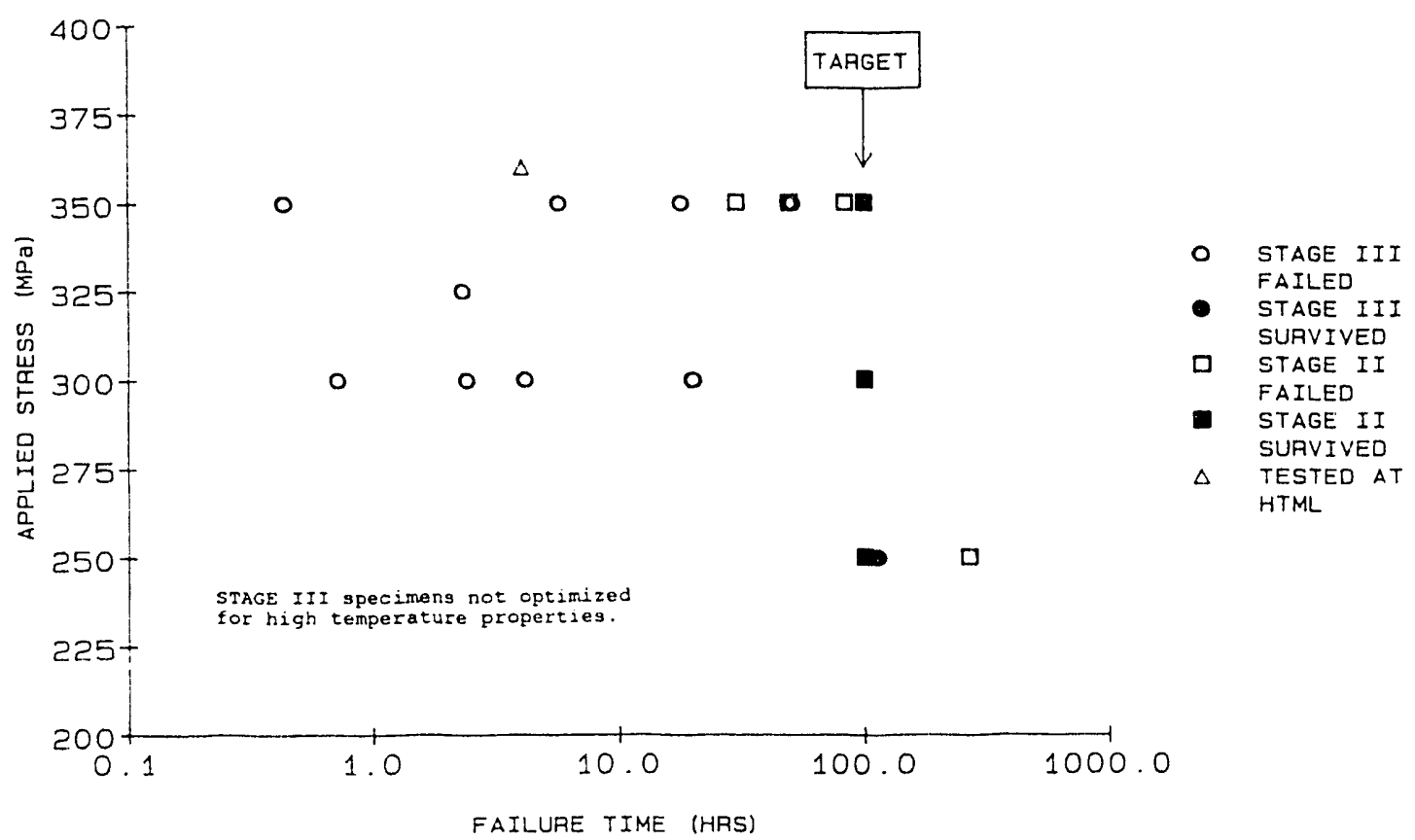

Figure 20: Tensile Stress Rupture Data at $1230^{\circ} \mathrm{C}$ in Air for stage II and Stage III specimens 


\section{LARGE CROSS SECTION SPECIMEN STRENGTH DATA}

The general applicability of the optimized process beyond NSF tensile rods was demonstrated by fabricating and testing $50 \mathrm{~mm}$ diameter, $150 \mathrm{~mm}$ long specimens. These have been referred to as MLP (machined from large piece) specimens, in that the specimens were machined into many flexure bars ( $3 \times 4 \times 50 \mathrm{~mm}$ ) for assessment of the properties across the $50 \mathrm{~mm}$ section. Excellent fast fracture strength results were obtained both at room and elevated temperatures. As was found for the intrinsic tensile strength data discussed above, the flexure data are well represented by 3 parameter Weibull models, Figure 21. Threshold flexure strength values of $653 \mathrm{MPa}$ and $517 \mathrm{MPa}$ are predicted by these models for room temperature and $1370^{\circ} \mathrm{C}$, respectively. The mean flexure strengths were $972 \mathrm{MPa}(n=30)$ and $561 \mathrm{MPa}(\mathrm{n}=33)$, respectively.
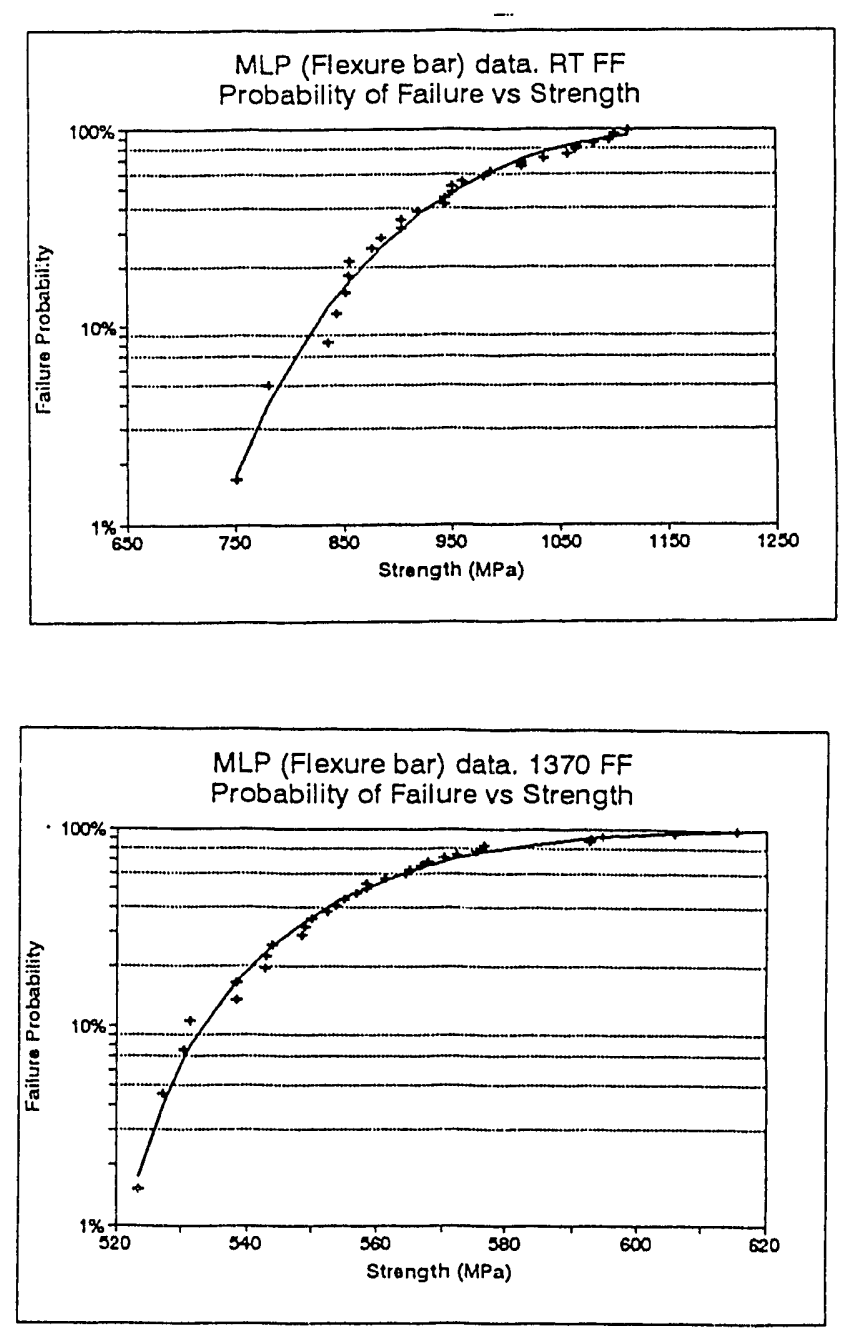

Figure 21: Flexure Strength Data from Large Cross section (MLP) Specimen 


\section{CONCLUSIONS}

Using an iterative processing approach, each unit operation of the colloidal consolidation process and the process as a whole has been optimized. Closed loop aqueous processing incorporating powder comminution, slurry concentration and casting has been the key to the successful completion of the program with desired reliability capability.

Equally significant has been the implementation of various powerful NDE techniques both for in-process control and for the identification of critical strength limiting defects. Use of MMR spectroscopy for slurry aging behavior characterization ${ }^{3}$, computed tomography (CT) for density gradient evaluation leading to optimum mold design, ${ }^{3}$ ultrasonic (UT) technique for in-situ monitoring of the casting process and density gradient evaluation in cast NSF bars", microfocus radiography for volume flaw detection both in green and dense specimens and lastly liquid dye penetrant (IDP) approach for the detection of machining related damage are a few salient examples.

A comprehensive database has been generated through fabrication, testing and post mortem fractography of a large set (>300) of net shape formed tensile bars. Analysis of the data suggests a significant reduction in both flaw size and the frequency of flaw occurrence. Microfocus radiography data suggests a five fold reduction in flaw size (from $250 \mu \mathrm{m}$ to $50 \mu \mathrm{m}$ ) and frequency of flaws $(2.5 / \mathrm{bar}$ to less than $0.5 / \mathrm{bar})$. This data agrees very well with the 1008 increase in average tensile strength realized in the program.

statistical analysis suggests that the truncation of large failure precursors affected by the processing strategy results in a three parameter Weibull strength distribution with threshold stress near 665 MPa. It follows that $\mathrm{NCX}-5102$ is both stronger and more reliable than the targeted material characterized by a mean tensile strength of 900 MPa and 2 parameter Weibull shape factor (modulus) of 20. 


\section{ACKNOWLEDGEMENTS}

Research sponsored by the U.S. Department of Energy, Assistant Secretary for Energy Efficiency and Renewable Energy, office of Transportation Technologies, as part of the Ceramic Technology Project. of the Materials Development Program, under contract DE-AC05-840R21400 with Martin Marietta Energy systems, Inc.

The success of the program resulted from the contribution of numerous individuals at Saint-Gobain/Norton, Department of Energy and Martin Marietta/Oak Ridge National Laboratory. Special acknowledgement is directed to Robert B. Schulz, Manager of the Materials Development Program, D. Ray Johnson, Manager of the Ceramic Technology Project and Ronald L. Beatty, Technical Monitor of this Processing for Reliability subcontract.

The four year program benefitted from the efforts of numerous S\&E staff members besides the authors. These included Ramal Amin, Dennis Creehan, Ajay Garg, stephen Hartline, Mike Mangaudis, Joe Panzarino, Julio Rossi, Param Tewari, stu Tuffs, Jon Wade and Charles White. The group of research assistants who diligently worked on the project included Dave Collette, Dave Goodmacher, William Hackett, Mirta Janniere, Gary Lambert, Rohert sliwoski, David Truedson and Greg Watson. The project team included an expert group of consultants and subcontractors who made significant contributions to the effort. Included in this group were Ilhan Aksay, Princeton University; Robert Botto, stephen Dieckman, William Ellingson and Nachappa Gopalsami, Argonne National Laboratory; Ronald Chand, Chand Rare Technical Ceramics; John Fraser, Precision Acoustic Devices; Chuck Hellier, Hellier Associates; Karl Jakus, University of Massachusetts; Fred Lange, University of California; Bryan McEntire, NTC Components; Dan Snoha and Mark Vangel, Army MTL; and Subbas Malghan, NIST.

The SG/NICC support team is acknowledged for their valuable assistance. This team included Frank Csillag, Tom DiMauro, Joy Garwood, Roberta Gordon, Marie Longbottom, Teresa Mursick-Meyer and Frederick Van slett. 


\section{REFERENCES}

1. V.K. Pujari, K.E. Amin and P.H. Tewari, "Development of Improved Processing and Evaluation of silicon Nitride," ASME paper 91-GT317, presented at Int' 1 Gas Turbine and Aeroengine Congress and Exposition, Orlando, FL, June $3-6,1991$.

2. S.F. Duffy, I.M. Powers and A. Starlinger, "Reliability Analysis of Structural Ceramic Components Using a Three-Parameter Weibull Distribution". ASME paper 92-GT-296, J. Engr. Gas Turbine and Power, 115, 1, Jan. 93, pp 109-116.

3. V.R. Pujari, D.M. Tracey, N.D. Corbin, M.R. Foley, et.al., "Reliability Improvement of High Strength Silicon Nitride Through Process optimization and Control," Procs. Automotive Technology Development CCM, Dearborn, MI, October 28-31, 1991, SAE, 1992, 137-144.

4. V.K. Pujari, I.C. Sales, J.S. Wang and J. Fraser, "Precision Casting Process Control by Ultrasonic Technique," Ceramic Engineering and Science proceedings, 13, No. 7-8, 1992, 536-545. 
1. V.R. Pujari, "Powder Characteristics and Their Effects on the Binder Removal Process," presented at the Third Int'l conf. on Ceramic Processing Science, San Diego, CA, February 4-7, 1990.

2. R. E. Amin, "Characterization of Processing Defects in Ceramics Using Microfocus Radiography," presented at the 92 nd ACS Annual Meeting, Dallas, Texas, April 22, 1990.

3. R.E. Amin, "The Use of Film Digitization Technology for Improving the Detection and Mapping of Defects in Ceramics," presented at the ASNT/ACS Conf. on NDE of Modern Ceramics, Columbus, OH, July 9. 1990 .

4. J.N. Panzarino, "NDE and Advanced Ceramics," presented at the ASNT ACS Conf. on NDE of Modern Ceramics, Columbus, OA, July 8, 1990.

5. V.K. Pujari et.al., "Development of Improved Processing and Evaluation of Silicon Nitride," presented at Automotive Technology Development CCM, Dearborn, MI, October 22-25, 1990.

6. J.D. Fraser, K.E. Amin and B.T. Kuri-Yakub, "High Frequency Ultrasonic Inspection of Green and BIP'ed Silicon Nitride Cylindrical Samples," presented at the TMS Annual Meeting on NDE, New Orleans, IA, February 16-17, 1991.

7. J.D. Fraser and K.E. Amin, "Novel Ultrasonic Techniques for Inspecting Si3N4 Ceramics," presented at ASNT spring Conf. on NDE, Oakland, CA, March 18-21, 1991.

8. V.K. Pujari, R.E. Amin and P.H. Tewari, "Development of Improved Processing and Evaluation of silicon Nitride," AsME paper 91-GT317, presented at Int'l Gas Turbine and Aeroengine Congress and Exposition, orlando, FL, June 3-6, 1991 .

9. V.K. Pujari, "Process Control in Injection Molding of Non-oxide Ceramics," presented at Powder Injection Molding Int'l symposium, Albany, NY, July 15-17, 1991.

10. N. Gopalsami, P. Rizo, W.A. Ellingson and D.M. Tracey, "Detection Sensitivity of X-ray CT Imaging for NDE of Green-state Ceramics," presented at Review of Progress in Quantitative NDE Conf., Brunswick, ME, July 28 - August 2, 1991.

11. V.R. Pujari et.al., "Reliability Improvement of High strength Silicon Nitride Through Process optimization and Control," presented at Automotive Technology Development CCM, Dearborn, MI, october 28 - 31, 1991 .

12. M.R. Foley and V.R. Pujari, "Tensile Testing in the Development of Processing Methods for High strength/High Reliability silicon 
Nitride," presented at the 16 th Annual Conf. on Composites and Advanced Ceramics, Cocoa Beach, FL, January 7-10, 1992.

13. V.K. Pujari, J.S. Wang, L.C. Sales and J.D. Fraser, "Pressure Casting Pressure Control by Ultrasonic Technique," presented at the 16th Annual Conf. on Composites and Advanced Ceramics, Cocoa Beach, FL, January 7-10, 1992.

14. A.R. Garg, V.R. Pujari, D. French and R. Botto, "NMRS Characterization of Ceramic slurry for Pressure casting," presented at ACS Annual Meeting, Minneapolis, MN, April 15, 1992.

15. V.R. Pujari, "Green Microstructure Control in the Colloidal Consolidation of Silicon Nitride," presented at the $23 \mathrm{rd}$ Annual Meeting of the Fine Particle Society/Symposium on Ceramics, Las Vegas, Nevada, July 16, 1992.

16. D.M. Tracey, "In-Process Inspection and Control for High Reliability Si3N4," presented at the DOE/ORNL Advanced Ceramic Manufacturing Workshop, Washington, D.C., September 22, 1992.

17. V.R. Pujari, "Development of Improved Processing and Evaluation Methods for High Reliability Structural Ceramics" presented at the Ceramic, Power Metal and Composite Manufacturing symposium, Worcester Polytechnic Institute, Worcester, MA, October 21, 1992.

18. V.R. Pujari et.al., "Processing Methodology for the Production of Reliable Bigh Strength Silicon Nitride" presented at the Automotive Technology CCM, Dearborn, MI, November 4, 1992.

19. D.M. Tracey et.al., "High Strength silicon Nitride Production Through Advanced Processing", presented at the MRS Fall Meeting, silicon Nitride Ceramics Symposium, Boston, MA, Nov.30-Dec.4,1992.

20. V.R. Pujari, L.C. Sales, and N.I. Paille, "Process Control Methods in the Consolidation of Highly Concentrated Suspensions," presented at the MRS Fall Meeting, silicon Nitride Ceramics Symposium, Boston, MA, November 30 - December 4, 1992.

21. M.R. Foley, et.al., "Silicon Nitride Tensile Strength Database from CTP Processing for Reliability Project", presented at ASTM Symposium on Life Prediction Methodologies and Data for Ceramic Materials in Advanced Technology Application - A Basis for Standards, Cocoa Beach, FL, January 12, 1993.

22. V.K. Pujari and P.J. Pelletier, "Ceramic Process Monitoring and Control by NDE Techniques," presented at the ASNT spring conf. and Second Annual Research Symposium, Nashville, TN, March 31, 1993.

23. V.K. Pujari and D.M. Tracey, "Processing Methods for Bigh Reliability Silicon Nitride Heat Engine Components," presented at ASME Turbo Expo - Land, Sea \& Air, Cincinnati, OH, May 24-27,1993. 


\section{PROJECT PUBLICATIONS}

1. V.R. Pujari, R.E. Amin and P.H. Tewari, "Development of Improved Processing and Evaluation of silicon Nitride," Procs. Automotive Technology Development CCM, Dearborn, MI, October 22-25, 1990, SAE, 1991, 133-142.

2. S.I. Dieckman et.al., "Three-Dimensional Nuclear Magnetic Resonance Imaging of Green-State Ceramics," Argonne National Laboratory Report ANL-91/39, May, 1991.

3. V.R. Pujari, R.E. Amin and P.H. Tewari, "Development of Improved Processing and tvaluation of silicon Nitride," ASME paper 91-GT317, presented at Int' 1 Gas Turbine and Aeroengine Congress and Exposition, 0..lando, FI, June 3-6, 1991.

4. N. Gopalsami, P. Rizo, W.A. Ellingison and D.M. Tracey, "Detection Sensitj.vity of X-ray CT Imaging for NDE of Green-state Ceramics," Review of Progress in Quantitative NDI, 11, D.O. Thompson and D.E. Chimenti, Editors, Plenum Press, N.Y., 1992, 1861-1868.

5. V.R. Pujari et.al., "Reliability Improvement of Bigh Strength silicon Nitride Through Process Optimization and Control," Procs. Automotive Technology Development CCM, Dearborn, MI, October 2831. 1991, SAE, 1992, 137-144.

6. M.R. Foley and V.R. Pujari, "Tensile Testing in the Development of Processing Methods for High Strength/Bigh Reliability Silicon Nitride," Ceramic Engineering and Science Proceedings, 13, No. 9$10,1992,978-990$.

7. V.K. Pujari, L.C. Sales, J.S. Wang and J. Fraser, "Precision Casting Process Control by Ultrasonic Technique," Ceramic Engineering and Science Proceedings, 13, No. 7-8, 1992, 536-545.

8. D.J. Snoha and M.R. Foley, "An Investigation of Residual Stresses in Machined Silicon Nitride," Army Materials Technology Laboratory TR 92-46, July 1992 .

9. V.R. Pujari et.al., "Processing Methodology for the Production of Reliable High Strength Silicon Nitride," to appear Procs.

Automotive Technc:ogy CCM, Dearborn, MI, November 4, 1992.

10. M.R. Foley, et.al., "Silicon Nitride Tensile Strength Database from CTP Processing for Reliability Project," presented at ASTM Symposium on Life Frediction Methodologies and Data for Ceramic Materials in Advanced Technology Application - A Basis for Standards, Cocoa Beach, FL, January 12, 1993, to appear ASTM STP 1201. 
11. V.K. Pujari and D.M. Tracey, "Processing Methods for Bigh Reliability silicon Nitride Heat Engine Components," presented at ASME Turbo Expo - Land, sea Air, Cincinnati, Ohio, May 24-27, 1993, to appear J.Engr. for Gas Turbines and Power. 
APPENDIX 1: MATERIAL SELECTION AND CBARACTERIZATION

MATERIAL SELECTION

A silicon nitride composition (with 48 yttria) was selected to perform the specified tasks and meet the required property goals. Ube silicon nitride powder was chosen for its purity because it was observed that many of the carbothermally produced powders (Remanord and stark) contain significant amounts of iron impurities which lower the strength of the densified materials as flaws. Ube Chemical was used as the source of silicon nitride throughout the program. This powder is a chemically derived product made by a thermal decomposition of silicon imide. The process begins with low temperature reaction semiconductor grade silicon tetrachloride with liquid ammonia to form silicon imide, followed by a first calcination step to form amorphous silicon nitride and second calcination step to form crystalline silicon nitride. The conditions used for the second calcination determine the particle size, surface area and morphology of the powder.

Three grades of Ube silicon nitride were used: Ube SN-E03, Ube SNE05, and Ube SN-E10. The blend ratio was adjusted to control packing density. The high purity raw materials resulted in a high purity powder. Low iron levels have been found necessary to obtain optimum high temperature properties. The alpha silicon nitride content of this powder is typically $>988$ and there is no measurable free silicon. Although residual chlorine can be detrimental to high temperature properties, our process has ensured a $c l$ level $<50 \mathrm{ppm}$. Residual carbon can be high for the ube product and is monitored, but no direct effect on final properties was determined.

Molycorp was the source of the yttrium oxide powder used in the co-milled process and the yttrium nitrate powder used in the precipitation process.

The above composition milled in alcohol showed potential for meeting the targeted room and high temperature properties. In this program alcohol milled powder was chosen to develop the baseline capability employing the injection molding process. Subsequently, the 48 yttria silicon nitride composition with process improvement was processed in an aqueous medium. Deionized water with a resistivity $\sim 18$ megohm-cm passed through a 0.2 micron filter was used throughout the program.

\section{MATERIAL CHARACTERIZATION}

silicon nitride stock powders were analyzed for chemical composition, impurities, and particle size distributions. Phase analyses were carried out by $x$-ray diffraction. Impurities were assessed by emission spectrographic analysis. The cationic impurities were determined by digestion of the powder and analysis by AA and ICP. The anionic impurities were determined by ion chromatography. Surface area and particle size distributions were determined by BET, sedigraph, and light scattering techniques to cover the range of fine particles. Analyses were carried out at each unit operation, and the tests required 
at each step, as well as the operational ranges were defined within the process SOP. Besides chemical analysis of powders, impurities and additives, a broad range of physical analyses were specified, e.g. particle size distribution, surface area, morphology, phases.

\section{Oxygen Analysis}

oxygen content in the silicon nitride powder was analyzed by the IECO method. The oxygen in samples combines with carbon th high temperature to produce $c 0$ which is analyzed by infrared detection. In the process which involved co-milling of Si3N4 and $\mathrm{Y}_{2} \mathrm{O}_{3}$ the detection of oxygen in the starting and in the milled powder was analyzed with fair certainty because the moisture content in the powder is small. Most of the oxygen in si3N4 is in the form of silicon oxide or dioxide. Also, the moisture/water content on such powders is determined by Rarl Fisher method and is always below 0.18 .

In the process which involved addition of the sintering aid by the precipitation technique, oxygen in the powder after yttria precipitation on the surface is a combination of oxygen generated during milling, the original oxygen in the powder, moisture content of the powder and the colloidally precipitated $\mathrm{Y}_{2} \mathrm{O}_{3}$. Therefore, two values of the oxygen are determined: 1) from the powder after controlled drying, which contains the contributions from all the sources discussed above and 2) after calcining the powder at high temperature in $\mathrm{N}_{2}$.

\section{Yttria and oxygen Values}

Due to the calcination used to produce yttrium nitrate, varying levels of residual carbon may be encountered and are monitored. Thermal gravimetric analysis of yttrium salt showed that the material has $6 \mathrm{H}_{2} \mathrm{O}$ molecules as specified. It decomposes to $\mathrm{Y}_{2} \mathrm{O}_{3}$ at $-700^{\circ} \mathrm{C}$ with a 708 weight 1088 . The formula used to specify the amount of yttrium salt needed to give 4 w8 $\mathrm{Y}_{2} \mathrm{O}_{3}$ has been modified. Instead of a factor of 3.5 , a factor of 3.4 was used. Also, the TGA for $Y(O B)_{3}$ showed that the full conversion to $\mathrm{Y}_{2} \mathrm{O}_{3}$ occurs between $800^{\circ} \mathrm{C}$ and $900^{\circ} \mathrm{C}$ with a 358 weight 1088 . Therefore the calcination of $\mathrm{Y}(\mathrm{OH})_{3}$ to $1000^{\circ} \mathrm{C}$ is needed to give a corresponding oxygen for $\mathrm{Y}_{2} \mathrm{O}_{3}$. However, the oxidation of $\mathrm{Si}_{3} \mathrm{~N}_{4}$ needs to be minimized. Various calcination treatments in vacuum and/or nitrogen to $800^{\circ} \mathrm{C}$ have consistently shown $0.5 \mathrm{w} / 0$ additional oxygen pick up.

The final value of the oxygen on the presintered bar as well as on the MOR bars after HIPing and machining has also been determined. For these determinations, the bar is crushed to small pieces, taking precautions that no contamination or oxidation occurs in the bar while crushing. These pieces $(50-60 \mathrm{mg})$ are used for LECO analysis.

Analysis of Metallic Species

The metallic species were extracted by acid digestion of the powder. The powder was digested first in sodium hydroxide. The fused material was then dissolved in hydrochloric acid. The yttrium analysis was performed on this solution by atomic absorption (AA) technique. The dissolved solution was also analyzed by inductively coupled plasma (ICP) 
technique. The choice of the equipment depends on the sensitivity of the determination of the element. For yttrium both AA and ICP were used; however, ICP gave a better sensitivity for the yttrium species. We have also determined yttrium by $x$-ray fluorescence (XFS). Emission spectroscopy technique has been used to analyze metallic impurities in the powder. This technique was used exclusively for aluminum, zirconium and iron, although the analysis is applicable to $\mathrm{B}, \mathrm{Ni}, \mathrm{Cu}, \mathrm{V}, \mathrm{Na}, \mathrm{Cr}$, $\mathrm{Ca}, \mathrm{Ti}, \mathrm{Mg}$, and $\mathrm{Li}$ as well. The anionic species in the powder was analyzed by ion chromatography. The material was digested and the solution analyzed for anionic species.

\section{Particle size and Surface Area of the Powder}

Both the surface area and the particle size distribution of the powders have been routinely measured to assess the extent of milling of $\mathrm{Si}_{3} \mathrm{~N}_{4}$ powders, while simultaneously measuring the oxygen content. The particle size distribution has been determined routinely by two techniques: 1) sedigraph and 2) microtrac. These two instruments combined give a reasonable picture of the size distribution of the particles. We measure $d_{10}, d_{30}$ and $d_{200}$ for a general comparison from batch to batch. The surface area is determined by multiple point BET gas adsorption using $\mathrm{N}_{2}$ as the gas. These two combined measurements give a reasonable state of the milled powder along with the oxygen values.

There was enough lot-to-lot variability in the ube powder to necessitate significant adjustment of milling conditions to achieve a consistent milled particle size distribution. Figure 1-1 shows the as received and milled average $d_{50}$ particle size for Lots $B, C$ and $D$. within a lot there was no significant variation. Figures $1-2$ and $1-3$ show the result of a harpoon analysis for the drums of ube silicon nitride powder of a single lot. This analysis indicates that although the differences in the variables of interest were significant when examining various drums within a lot, the differences within a drum were insignificant.

\section{Rheological Measurements}

Rheological measurements on the slurry have been performed by Bohlin rheometer model VOR and constant stress rheometer C.S. These two units together provide capabilities to analyze slurries completely in viscometry, oscillatory and relaxation tests. Constant stress rheometer also gave accurate values for the yield stress often used tc interpret rheological data and their implications. The Injection molding compound was fully characterized rheologically using the capillary rheometer.

\section{IEP Measurements}

Another colloidal property monitored on incoming Ube powders was IEP done by Pen Rem 7,500 acoustophoretic technique which not only determines the charge on the particles but also assesses the role of impurities and their extent on the powder surface. Lot $\mathbf{E}$ showed a significant difference in IEP from lots A through D (see Appendix 2). various treatments were attempted to effect a change in the IEP with 
varying degrees of success on a laboratory scale. Further scaleup optimization of these treatments is required. Prior to receipt of Lot $F$ powder, several preshipment samples of various ube lots were analyzed for isoelectric point and particle size distribution. The lowest isoelectric point Ube lots were selected for Lot $F$ shipment.

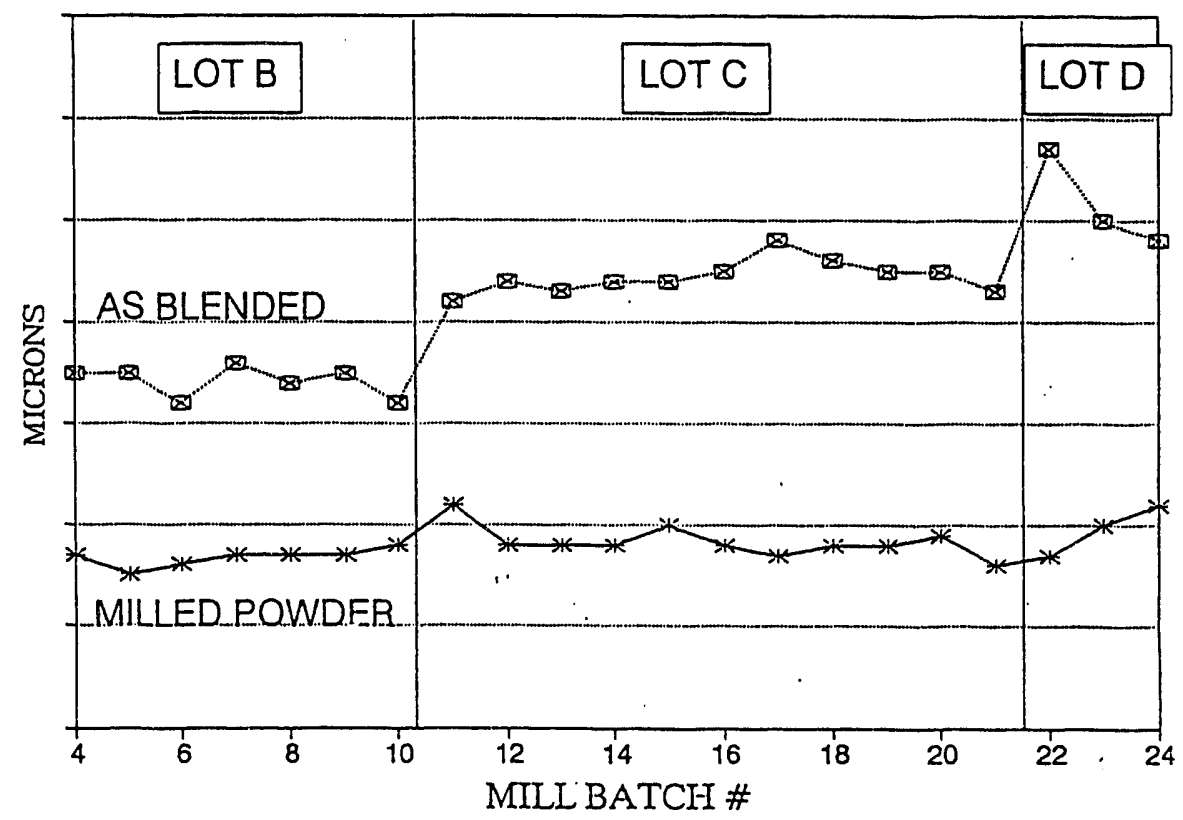

Figure 1-1: $D_{50}$ Particle Size for As-received and Milled Powder Batches

\section{Milling media}

Submicron debris was a component of the final milled powder due to attrition during milling. The attrition rate varied slightly depending on conditions, but ranged from 0.2 to $1.0 \mathrm{w} / 0$ of the final milled powder. The media is basically the same composition as the powder, but since it has been densified by HIP'ing, it consists of beta silicon nitride. This submicron beta silicon nitride may have an influence on seeding for grain growth during HIP densification of the product.

\section{CHARACTERIZATION OF THE MILLED SILICON NITRIDE SLURRIES}

The water milled silicon nitride slurry, in the presence of the surfactant, has a pH of $9.0+.1$ and the isoelectric point of the powder is $5.5+.1$. Therefore, the milled slurry is a well deflocculated stable suspension, ideal for filtration. After filtration the entrapped material in the filter and the filtered slurry powders were analyzed for particle size distribution by SEM and Sedigraph and by BET measurements for surface area.

These measurements confirm that the slurry is indeed very well deflocculated. The SEM and sedigraph data show that $d_{10}, d_{30}, d_{90}$ for the particles do not change significantly by these filtrations. Also, more 
than 938 of the slurry is recovered by this filtration.
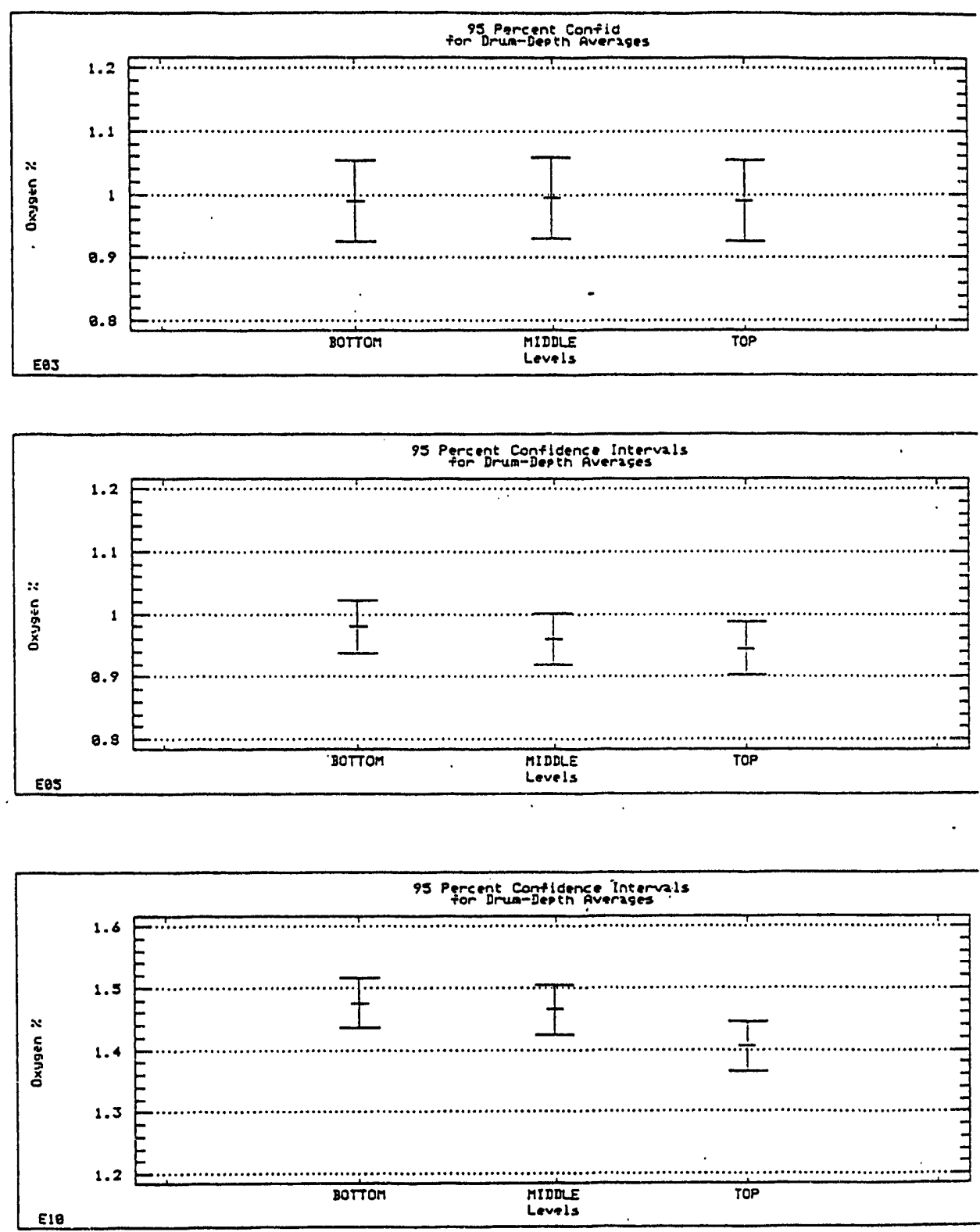

Figure 1-2: Oxygen Level in As-received E03, E05, E10 Ube Powder

Surface impurities of the milled powders were characterized by $x$-ray photoelectron spectroscopy. The starting powder and the powder after milling with different amount of the surfactants were examined for surface impurities. The XPS data show less than two atomic percent aluminum and no detectable $\mathrm{zr}$ impurities in the starting as well as milled powders. However, ICP and emission spectrographic analysis show different levels of aluminum and $\mathrm{zr}$ in the milied powder. The vaiues of $\mathrm{Al}$ and $\mathrm{zr}$ in the powder by emission spectrographic analysis are 
significantly higher than those of ICP values. The reason for this discrepancy has not yet been identified.
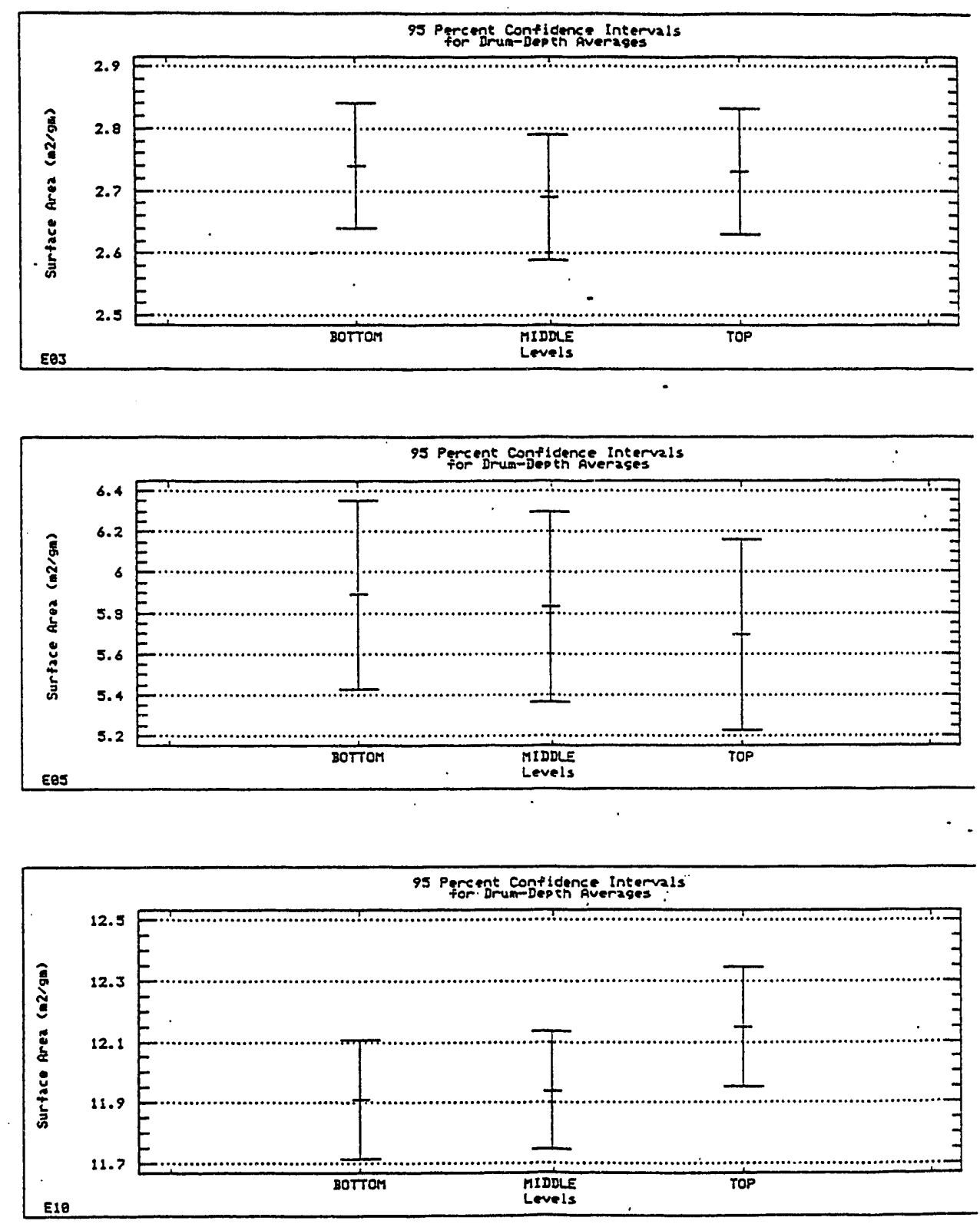

Figure 1-3: Surface Area of As-received E03, E05, E10 Ube Powder 
Thermal analysis was regularly used for the analysis of binders and plasticizers. More specifically, injection molding binders were measured for thermal conductivity, specific heat (DSC), glass transition temperature (DSC), and pressure-volume-temperature (p-v-t) correlation. Differential scanning Calorimetry (DSC) and Gel permeation Chromatography (GPC) techniques were used to characterize the virgin binder and the molding compounds. Melting peaks and the heat of transition for the virgin binder and the molding compounds were established for repeated heating cycles. These data were used to monitor and control the binder characteristics at various stages of the processing. These properties have also been used for the finite element analysis of the injection molding process. DSC and Gel Permeation Chromatography techniques were also utilized to characterize the virgin binder and the molding compound for $\propto C$ purposes. Due to the experimental difficulties (caused by powder loading) GPC could not be performed on the molding compounds.

\section{CHARACTERIZATION OF CASTING PLASTER MOLDS}

Plaster molds used in the pressure casting process were characterized both chemically and physically. Chemical analysis determined metallic impurity levels by emission spectroscopy. Physical characterization consisted of mercury porosimetry measurements. 
APPENDIX 2 : MATERIAL PROCESSING AND PROCESS CONTROL

POWDER PROCESSING

All process equipment were set up inside a Class 10,000 hard-wall clean room, specifically constructed for this program. The room was divided into a vestibule (for dressing into clean room garments and monitoring of conditions such as temperature, humidity and pressure), a powder storage room and the main processing room. Pressure was maintained highest in the main processing room. A vibratory mill was set up for both semicontinuous operation (14 to $60 \mathrm{~kg})$ and batch operation $(4-11 \mathrm{~kg})$. High purity silicon nitride milling media were specially fabricated for this program. The media were shaped as cylindrical bodies with hemispherical ends. Vibration milling was used as compared to ball milling because it provides for continuous monitoring and sampling throughout the milling cycle. Lower solids contents can be milled without the excessive media wear associated with the higher impact forces of ball or attrition milling.

Except for the initial process iteration, water milling was used instead of conventional alcohol milling. Water milling had in the past been thought to promote hydrolysis and formation of surface oxide layers so that alcohol was the preferred medium for milling. The powders milled in alcohol contain at most a trace or no hard agglomerates at all and the soft agglomerates break up easily. In the presence of alcohol, the decomposition and/or hydrolysis of silicon nitride should not proceed actively. Silicon nitride powders milled in water are known to contain higher levels of hard agglomerates and oxygen for two primary reasons: post-milling processing steps (oven drying) and decomposition/hydrolysis of silicon nitride in the presence of water. We have been able to successfully mill silicon nitride powder in water by controlling the chemistry of the aqueous phase using surfactants. Aqueous milling grants a number of benefits such as lower cost, environmental compatibility and process flexibility. Given a postmilling concentration step, a very low solids content can be used which allows for filtration at smaller sizes and more efficient magnetic separation.

The primary function of filtration of the slurry was to remove agglomerates and any foreign objects than may have been introduced during milling and handling of the powder. If a slurry containing primarily minus 1 micron particles is filtered, then filtration at 10 , 20, 30 micron should not present any problems. However, as the filtration size is decreased to 10 microns and finer, increasing resistance to filtration is observed due to increasing number of nearsize particles. Therefore, a requirement for filtration to succeed is a well dispersed slurry. In this program, filtration sizes ranging from 2 to 80 microns were evaluated. The use of water as the medium allowed for dilution of the slurry so that filter sizes less than 10 microns could be successfully used. Magnetic separation of the milled slurry was performed using a high gradient magnetic separator. The slurry was recirculated through the separator for a fixed period of time.

After the low solids content slurry was filtered, a concentration step was used to dewater the slurry to achieve a high solids loading 
that could be directly cast. Solids loadings of 65 to 74 w/o were easily achieved. Loadings above 748 were limited due to dilatency. Process equipment such as pumps can impart a higher shear rate than normally measured using a Brookfield viscometer, resulting in dilatency. Another advantage of this dewatering process was to decrease the level of residual $\mathrm{Cl}$ and $\mathrm{F}$ that could exist on the as-received Ube silicon nitride powder. Chlorine has been shown to be detrimental to high temperature properties.

\section{W-Series Process}

Initially, the sintering aid (yttria) was added by a precipitation of salt process, Figure 2-1. After aqueous milling and filtration the slurry was pumped in a closed loop to a precipitation tank. A yttrium salt was then added and the $\mathrm{pH}$ adjusted in a controlled manner to provide for precipitation of the hydroxide on the surface of the powder. The soluble product salt was then rinsed from the slurry to a level less than $50 \mathrm{ppm}$. The precipitated slurry was floccr iated at this stage. A dispersant was added and the slurry concentrated to a level appropriate for casting, typically 72-74 w/o. The surface of the powder resulted in dispersion behavior similar to yttrium hydroxide. This approach had several advantages and disadvantages. The resulting slurry was extremely stable. Because the yttria was present as a hydroxide, there was no aging problem such as encountered with a co-milled route. Also, the surface hydroxide resulted in a partially flocculated slurry, and therefore easily redispersed after settling occurred during storage. slurry produced by this method has been found to have a shelf life of months. In contrast, the co-milled product must be used within days and must be agitated continuously during that period. Due to the hydroxide, there would typically be a higher oxygen content $(\sim 0.58$ higher) of the product due to hydrothermal oxidation of the silicon nitride by the yttrium hydroxide. Although the process worked well on small batches $(4 \mathrm{~kg})$, on scaling up to $30 \mathrm{~kg}$ batches there were yttrium hydroxide agglomerates $(100 \mu \mathrm{m})$ in the product found to be caused by improper control of the salt concentration, causing supersaturation, Figure 2-2. By modifying the process to obtain a constant salt level throughout the precipitation process, the agglomerates were eliminated, Figure 2-3. However, difficulties were then encountered in concentrating the slurry. Due to time restraints the precipitation method was abandoned in favor of a more reproducible comilling process.

\section{C-Series Process}

The co-milled process (C-Series) was developed in response to scaleup problems with the W-series and became the standard process for the program. The silicon nitride powder was co-milled with a yttrium oxide powder, Figure 2-4. Significant foaming was initially encountered and eliminated with suitable surfactants. The surfactant system was complicated by the two component system. Filtration was more difficult as compared to the $\mathrm{W}$-series in which only silicon nitride is filtered. The final rheology of the siurry was much different from the w-series, with a much lower viscosity being obtained at a given solids loading. 


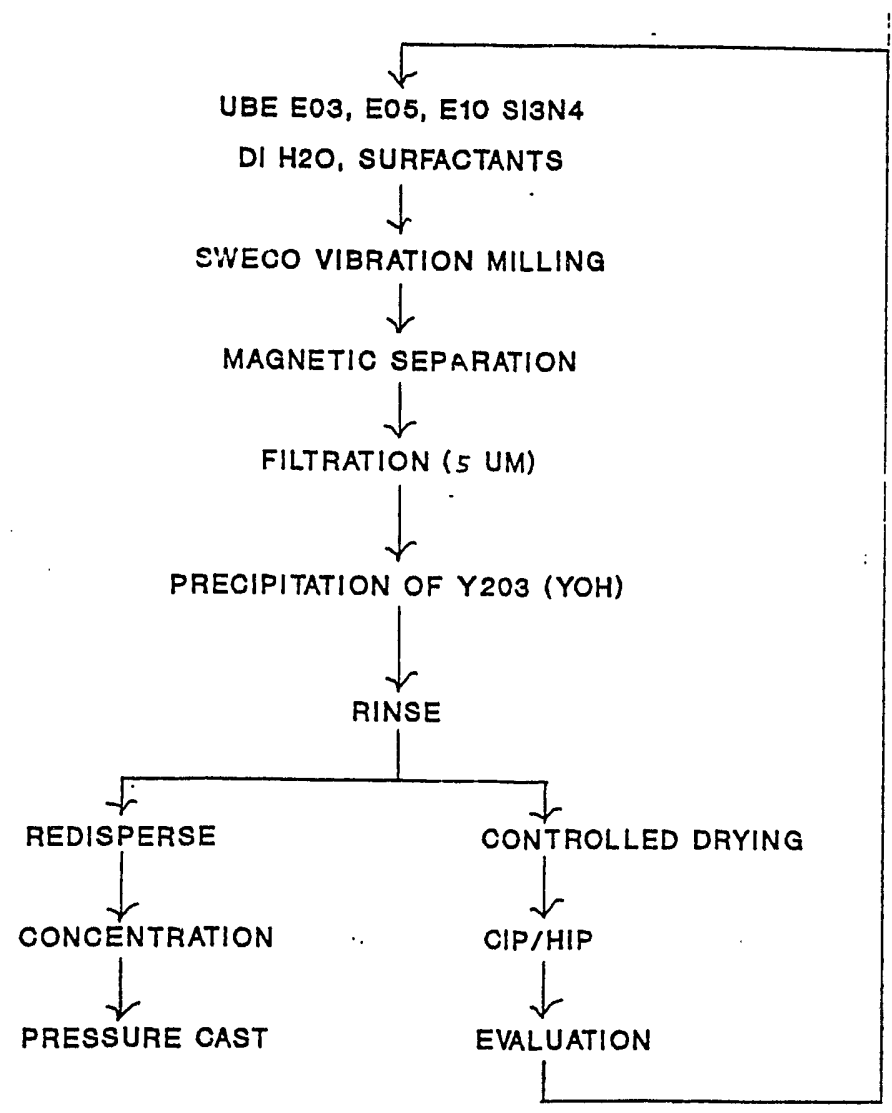

Figure 2-1: Process Flow Chart, W-Series

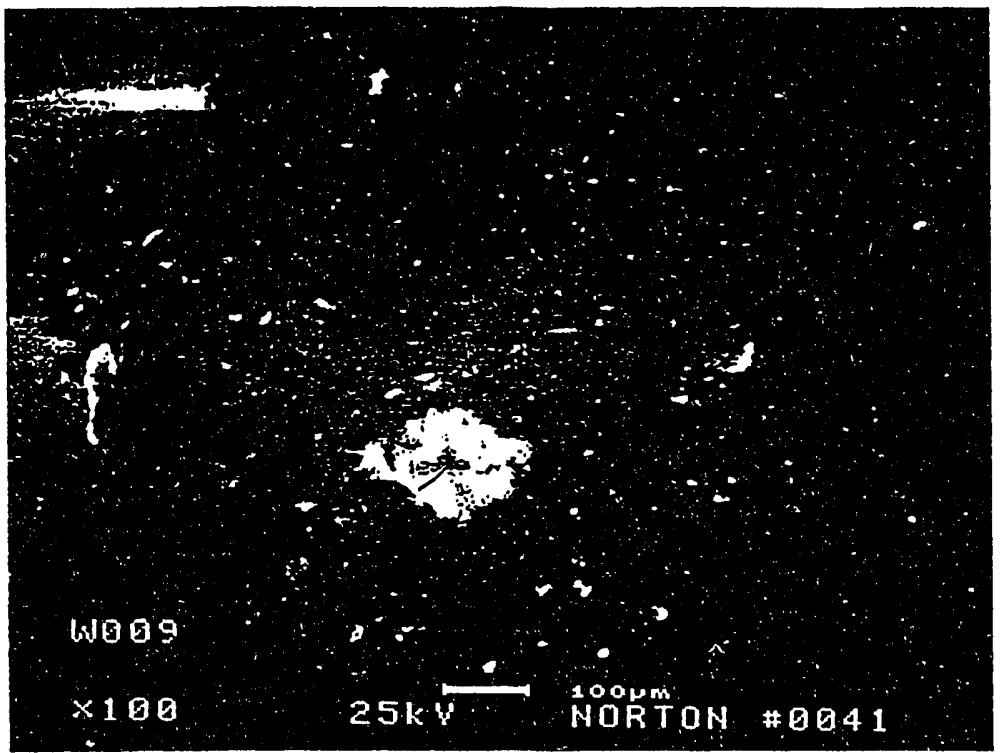

Figure 2-2: Baseline Precipitation 


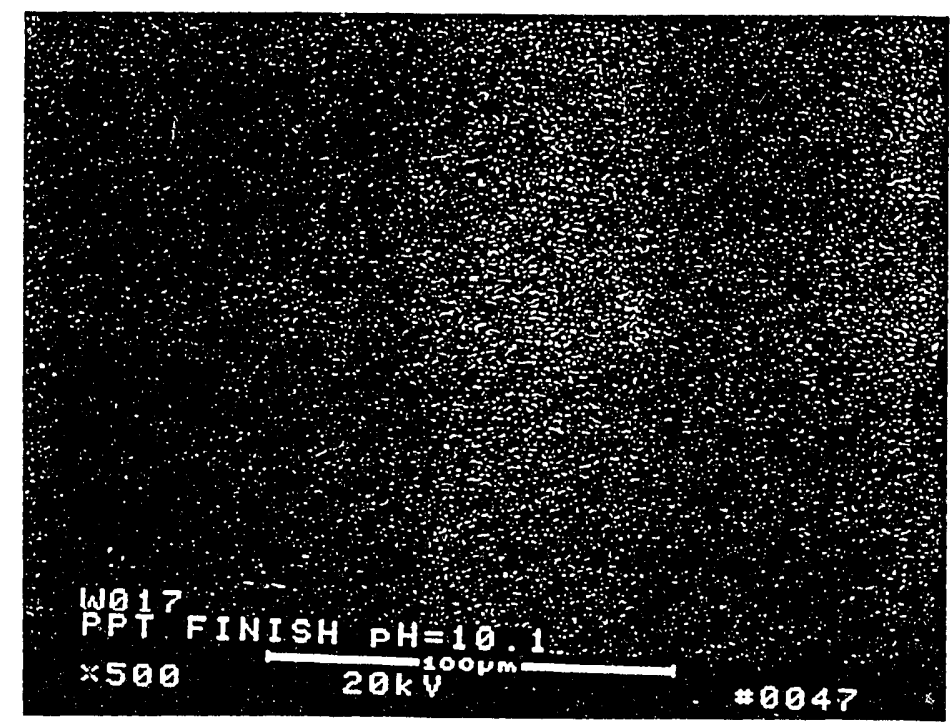

Figure 2-3: Modified Precipitation

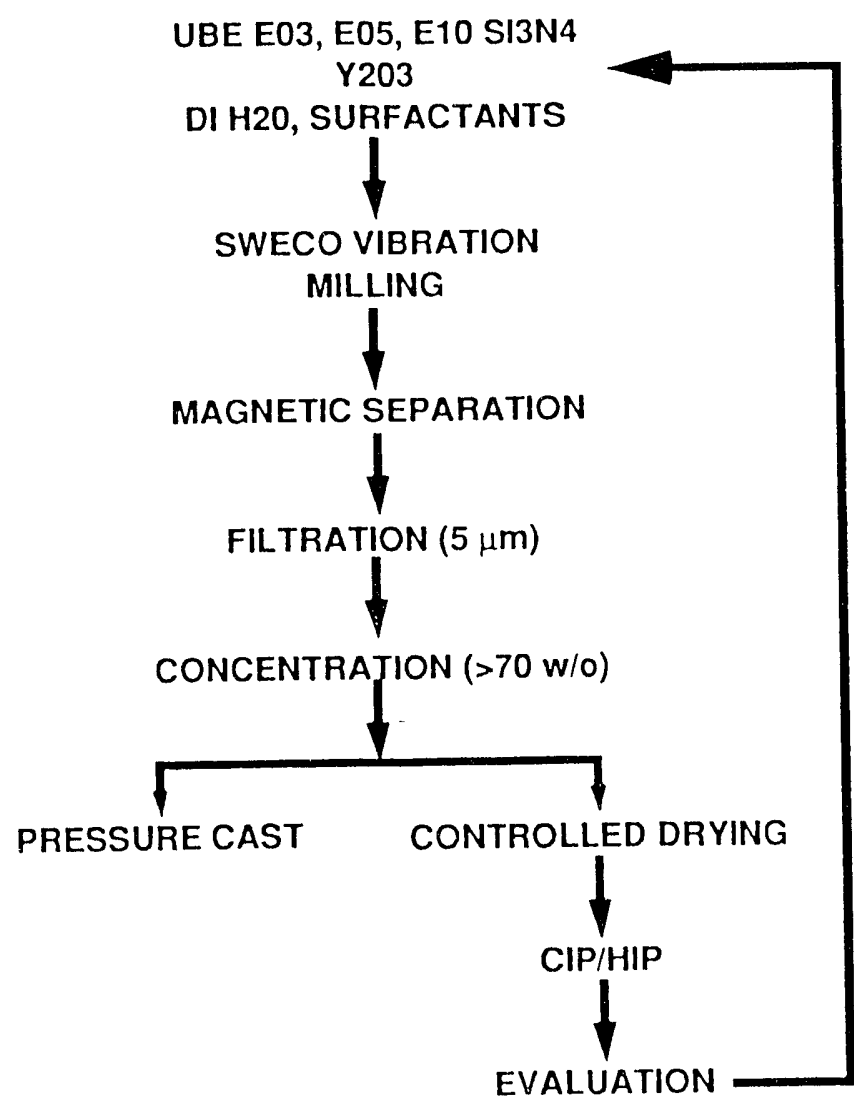

Figure 2-4: Process Flow chart, C-series 
PROCESS CONTROL

soc Control Charts

Shewhart control charts were employed for tracking mean and variance of key variables under optimized conditions for which upper and lower limits were established. Table 2-1 shows the variables initially monitored and those added during the program in response to issues encountered in control of downstream process properties. A statistical quality control software package was implemented with the ability of providing programmed indication of out-of -Control (OOC) conditions (socpack/Plus, PQ Systems, Inc., Dayton, ohio). Control limits $( \pm 3 \sigma)$ and specifications were established for the milled powder properties.

Table 2-1: Process Control Variables

\begin{tabular}{|l|l|}
\hline ORIGINAL & ADDITIONAL \\
\hline Oxygen & D10, D90 \\
\hline Surface Area & PH \\
\hline Yttria & Viscosity \\
\hline D50 & IEP, Cast Rate \\
\hline Fracture Toughness & Specific Gravity \\
\hline & Packing Density \\
\hline
\end{tabular}

In order to monitor the powder processing independent of forming issues, aqueous milled powder was freeze dried, screened, preformed and CIP'd as tiles. Tiles were HIP'd for each batch of powder prepared. Test bars were machined and tested for strength and fracture toughness. Fracture toughness eventually became a key control parameter for the powder, Figure 2-5. Fracture toughness values were higher for this water milled process than baseline properties (6.3 vs $5.4 \mathrm{MPa} / \mathrm{m}$ ). Packing density was another measurement that was obtained independert of the forming process using a preformed and cIp'd pellet. Samples of the powder from each of the powder lots were archived for future reference.

\section{Quality Assurance}

Calibration, standards and sampling of powder specimens have been discussed in Appendix 1. A detailed sop was written for the powder processing operation. The analytical methods employed to analyze powder and slurry properties are listed in Table 2-2. 


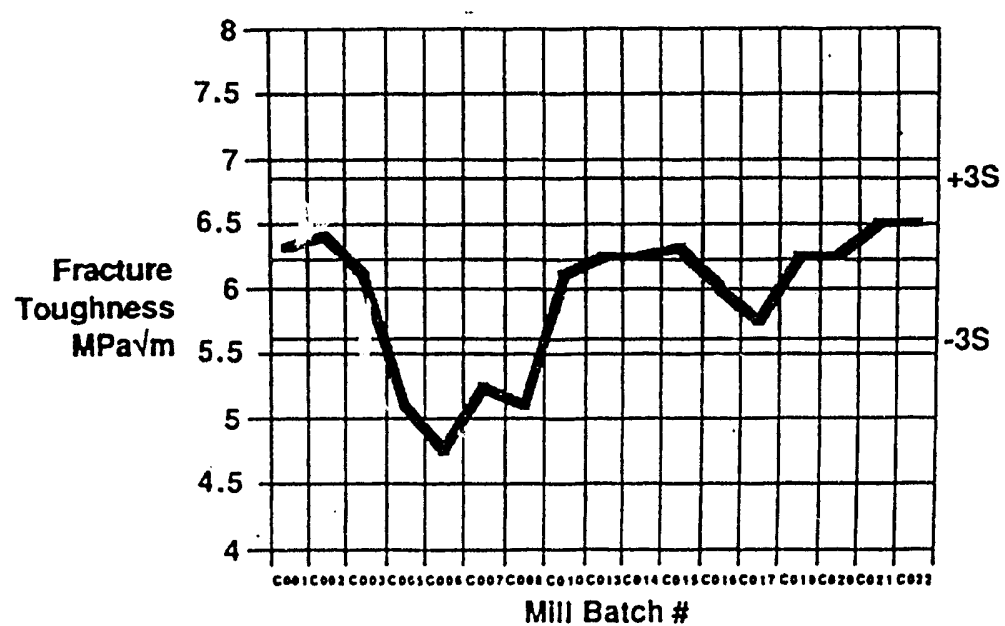

Figure 2-5: Fracture Toughness of Qualification Tiles

Table 2-2: Powder Characterization Techniques

\begin{tabular}{|l|l||}
\hline \multicolumn{1}{|c|}{ MEASUREMENT } & \multicolumn{1}{c|}{ TECHNIQUE } \\
\hline Particle Size & $\begin{array}{l}\text { Sedimentation (Sedigraph 5101) } \\
\text { Laser Scattering/(Malvern) } \\
\text { Photon Correlation } \\
\text { Spectroscopy }\end{array}$ \\
\hline Surface Area & BET Gas Adsorption \\
\hline Cation Impurities & Emission Spectroscopy \\
\hline Anion Impurities & Ion Chromatography \\
\hline $\begin{array}{l}\text { Oxygen } \\
\text { Carbon }\end{array}$ & Leco IR Adsorption \\
\hline IEP/Conductivity & $\begin{array}{l}\text { Acoustophoretic Mobility } \\
\text { (Pen Kem 7000) }\end{array}$ \\
\hline Yttria & Induction Coupled Plasma \\
\hline Surface Chemistry & $\begin{array}{l}\text { ESCA } \\
\text { FTIR }\end{array}$ \\
\hline Moisture & Karl Fischer Titration \\
\hline Phases & $\begin{array}{l}\text { X-Ray Diffraction } \\
\text { NMRS (Xstallinity) }\end{array}$ \\
\hline
\end{tabular}

\section{Milling Control}

Lot-to-lot as received powder variability required adjustment of milling conditions to achieve the same average particle size. Sedigraph 
measurements were obtained at different times in the milling cycle. Figure 2-6 shows how the average particle size varied lot-to-lot at the beginning of the milling process and how by adjustment of the milling conditions a consistent final milled average particle size was achieved. Solids content also allowed for control over the size distribution, with higher solids contents resulting in a broader size distribution, with a decreased D10 (8 finer than).

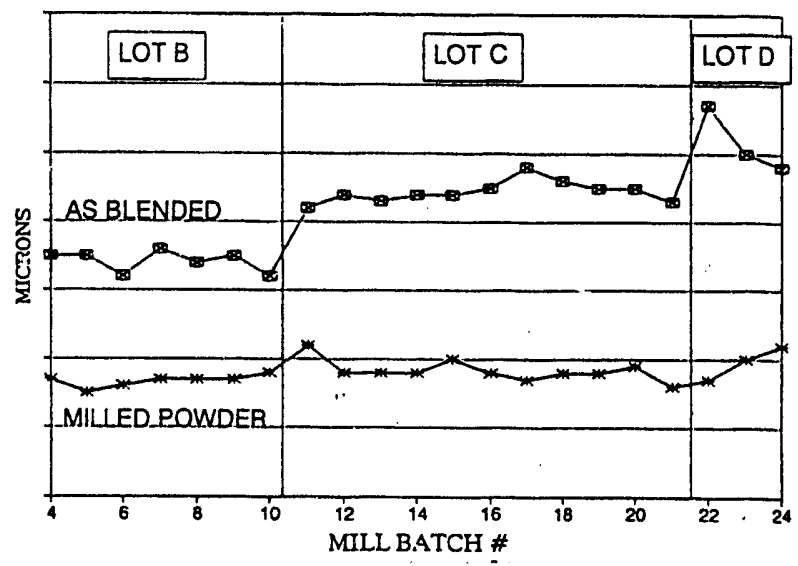

Figure 2-6: Chart for Particle Size - D50 For C-Series Mill Batches

\section{Surface Chemistry}

The casting rate characteristic of each batch of slurry was

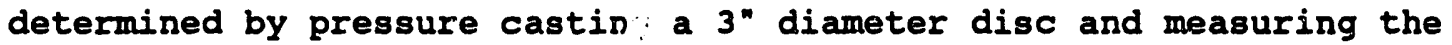
thickness as a function of time. The slope of the plot of thickness squared vs time was taken as an indicator of casting rate. Figure 2-7 shows the casting rate slopes obtained for each slurry batch produced from UBE powder Lots $D, E$ and $F$. A slope of less than 5 indicated $a$ slurry that would have a slow casting rate, high cast density and acceptably low warpage during HIP'ing. Casting slopes for Lot E powder were unacceptably high, resulting in unacceptable warpage (runout) during HIP'ing. There was a somewhat narrower size distribution obtained from Lot $E$ powder, resulting in a slightly lower CIP'd packing density, but not enough to account for the significant decrease in cast density.

The surface charge of the slurry was measured as a function of $\mathrm{pH}$ using both a PenKem 7000 and by Dr. Subhas slalghan at NIST using a Matec 800. The ESA (electrokinetic sonic amplitude) measurement using the Matec 800 showed the Lot $E$ katches to possess a significantly higher isoelectric point than for Lot $D$ batches. This was also the case for the starting Ube Si3N4 powders. These measurements were supported by measurement of the isoelectric point using the PenRem 7000 .

In order to improve the surface chemistry, chemical rinsing and thermal treatments wure evaluated. These treatments were successful on smaii batcines (ẑûg), decreasing the isoeiectric point significantiy and in most cases improving the casting behavior even relative to the Lot $D$ untreated powider. 


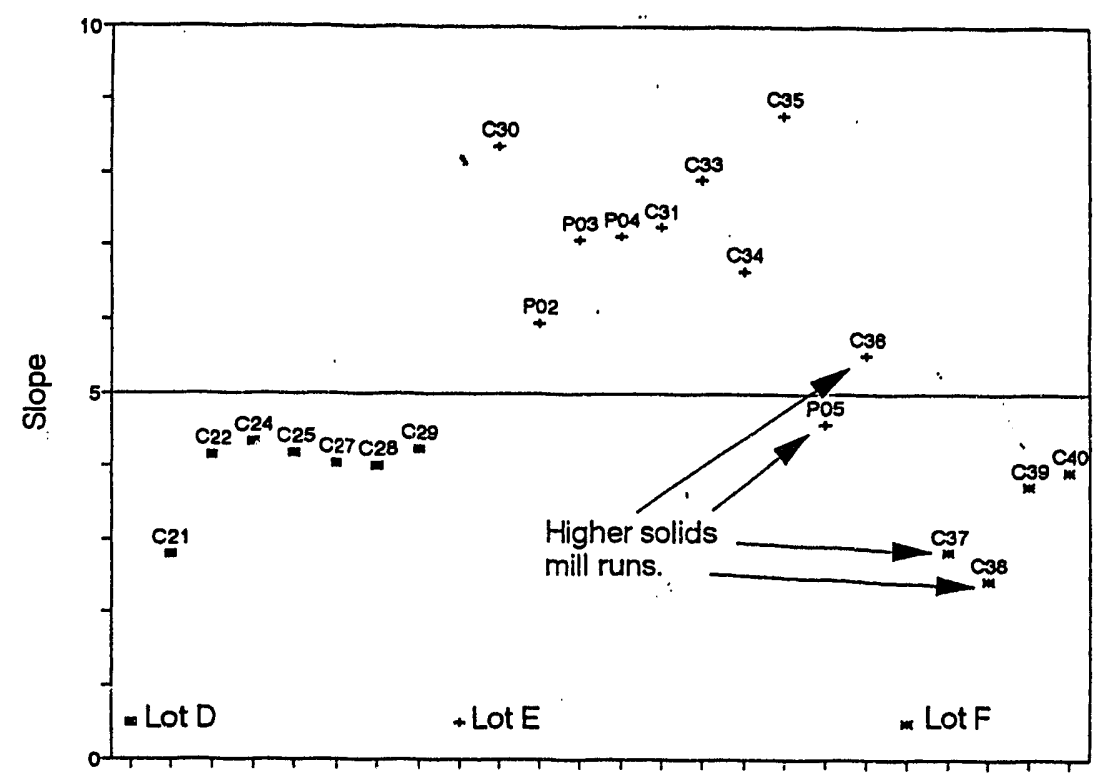

Figure 2-7: Casting Rate Slope for Lot D, E\& F Mill Batches

FTIR analysis of the powder before and after the treatments showed a significant change in the relative amounts of silanol and amine surface species. The treatments either decreased the amine species, leaving the same silanol species or significantly increased the silanol species. Rinsing treatments (Batches $\mathrm{c035}, \mathrm{C036}$ ) and a thermal treatment (Batch C043) were evaluated on large batches, only incremental improvements in the casting slope were obtained, which was supported by only a slight decrease in the isoelectric point. Further scaleup optimization of these treatments is required.

Prior to receipt of Lot $F$ powder, several pre-shipment samples of various ube lots were analyzed for isoelectric point and particle size distribution. The lowest isoelectric point ube lots were selected for Lot $F$ shipment. The milled Lot $F$ powder was acceptable in casting rate, cast density and warpage. The isoelectric point was also acceptably low.

The oxygen content of the milled powder decreased for Lots $\mathrm{E}$ and $F$, causing the process to be out of control and being very close to the lower limit specification for oxygen. The decrease in oxygen was caused by a decrease in the oxygen content of the ube silicon nitride powder for these lots. The need for a broader particle size distribution (coarser E03) and selection of an optimum isoelectric point resulted in the selection of the Lot $F$ powder from a number of pre-shipment samples. The thermal treatment used for Batch 043 resulted in the oxygen content being increased to an acceptable level. Due to the effect of the ube silicon nitride on the final milled powder properties it was determined that ravised specifications are required to ensire a controlled milling and casting process. These revised specifications include isoelectric point, oxygen content and particle size distribution. 
Inclusion/Agglomerate Free Product

The baseline process iteration failure origins were mainly iron inclusions resulting from the equipment used for injection molding. Iron inclusions were also a major failure origin in the baseline alcohol milled cIP'd rods. Therefore, the milling process was designed to eliminate the potential for metallic inclusions, especially iron. Features included processing within a class 10,000 cleanroom, separation of dry powder from the milling area, use of filtered and deionized water, magnetic separation and filtration of the milled slurry, use of a closed loop system from milling to casting, non-metallic materials of construction throughout the milling and casting, high purity/low attrition HIP'd milling media of the same composition, and use of microfocus $x$-ray to monitor inclusions at each step of the process. The final iron level in the milled powder was $<1 \mathrm{ppm}$ as measured by emission spectroscopy.

Metallic inclusions from 25 to 200 microns in size were detected by microfocus $x$-ray, in the in-coming ube silicon nitride powder. The frequency of inclusions did vary lot to lot, with no inclusions detected in the final Lot $F$ powder. The final milled slurry did not possess any metallic inclusions as measured by microfocus $x$-ray, to within the $25 \mu \mathrm{m}$ limit for this technique. Failure origins in some final HIP'd bars consisted of fine regions of iron silicide that were 1-5 microns in size, which linked to form a failure origin as large as 100-200 microns, Figure 2-8. These linked Fesix based regions are not always detectable by microfocus $x$-ray.

An increase in metallic inclusions as failure origins was experienced for Iteration $O$ through $R$. The source was traced to a piece of equipment used in the pressure casting scaleup. This source was eliminated as well as every other potential source of metallic contamination throughout the process. The frequency of metallic inclusions detected by microfocus $x$-ray was imnediately decreased, Figure 2-9. There were no iron related failure origins in the second half of stage II and throughout stage III.

\section{Milling Optimization}

The use of appropriate surfactants allowed the control of oxygen content to levels no higher than those obtained for alcohol milling. The closed loop system eliminates the need for drying and redispersion, thus decreasing the potential for inclusions and agglomerates.

A study was conducted in stage II to determine the optimum surfactant for milling and casting. This study showed that the oxygen could be controlled, the milling rate improved and the cast density improved with alternative surfactants. However, a drying related cracking problem was experienced with the alternative surfactants. Therefore the baseline surfactant was retained for stage III. Using alternative surfactant, the particle size distribution as measured by the sedigraph 5100 was found to be identical to the baseline surfactant, however the surface area was significantly higher. The Sedigraph 5100 does not resolve well below 0.2 microns. A Malvern system $4700 \mathrm{c}$ submicron particle size analyzer was evaluated, but could not resolve the 
differences on the as milled slurries due to the broad size distribution.
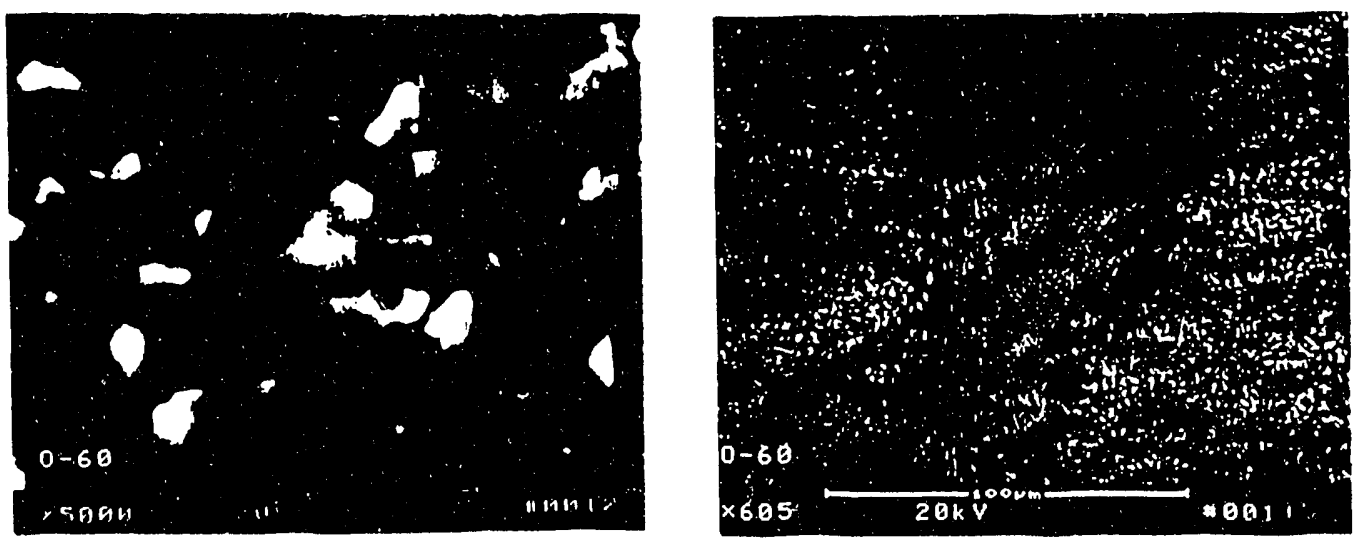

Figure 2-8: Fine Scale Iron Silicide Aggregate Failure Site

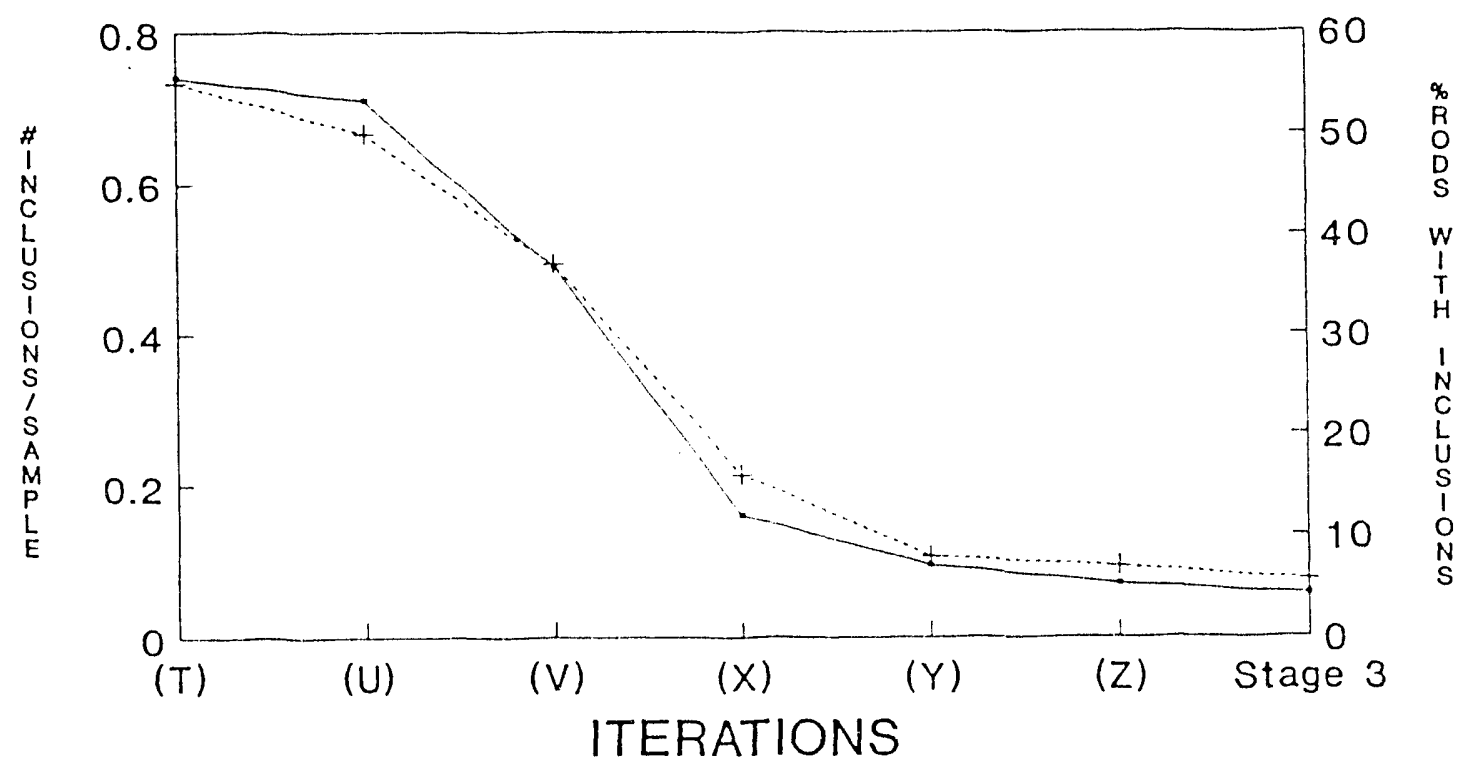

$\cdots$ \#INC./SAMPLE $\cdots+\cdots$ \%RODS W/INC.

Figure 2-9: Microfocus X-ray Flaw Detection of Inclusions in HIP'ed Rods

A study was conducted to determine the effect of solids content on the vibration milled powder. Higher solids loadings were shown to decrease the D10 (108 finer than) and increase the D9o (908 finer than) as measured by the sedigraph 5100. This resulted in a slightly higher packing density. The efícct of solids content on milied particle size distribution provide some control over the effect of ube silicon nitride powder particle size variability. 


\section{COLLOIDAL CONSOLIDATION PROCESS DEVELOPMENT}

The colloidal consolidation (Pressure Casting) process development was performed utilizing concentrated aqueous suspensions described in the previous section. Both the precipitated (W-series) and co-milled (C-series) suspensions were evaluated for their casting behavior and resultant tensile strengths. C-series suspensions, in general, exhibited greater reproducibility and improved mechanical properties. Consequently, C-series suspension was selected to conduct all casting experimentation and optimization in the program.

\section{Slurry Development and Characterization}

The milled and concentrated batches described in the previous section were conditioned following the established sOP. SOP included sonication with an ultrasonic horn for five minutes, followed by deairing in vacuum $(60 \mathrm{~mm}$ of $\mathrm{Hg})$. In addition, the slip was characterized for agglomerate population using an agglomermeter gage. Approximately ten samples per casting batch were examined to ascertain the agglomerate population in the slip.

A comprehensive aging study was conducted for water milled silicon nitride slurry for a period of 24 days following the concentration of the milled batch. The investigation focused on establishing the causeeffect relationship involving slurry behavior and certain basic slurry parameters. Measurements of viscosity, $\mathrm{pH}$, centrifuged sediment packing density and hard agglomerate size were made daily for the first 5 days, and then at one week intervals. simultaneously, NMR longitudinal relaxation time (T1) and spin-spin transverse relaxation time (T2) measurements were conducted at Argonne National Laboratory. viscosity (Brookfield, 30 RPM) of the slurry remained at $22 \mathrm{cp}$ during the aging. Largest agglomerate size as measured by a gage was relatively constant at $25 \mu \mathrm{m}$. The slurry $\mathrm{pH}$ was found to increase from an initial value of 9.8 to final value of 10.4 , and the packing density of the sediment (obtained by centrifuging the slurry for $30 \mathrm{~min}$. at $\sim 1000 \mathrm{~g}$ ) was found to increase from $\sim 57$ to $60.5 \mathrm{v} / 0$ during the 24-day aging period, Figure 2-10.

The changes in the proton NMR longitudinal relaxation time are given as a function of aging time in Figure 2-11. In general, a reduction in the $T 1$ value is observed with increasing aging time. During the initial stages, the $T 1$ demonstrates oscillatory behavior. It subsequently stabilizes at $\sim 680 \mathrm{~ms}$ after 13 days of aging. The decrease in $T 1$ parallels the increase in the centrifuged sediment density. This suggests that dynamic chemical processes govern aging of the slip initially, reaching an equilibrium state in about two weeks. A general increase in the $T 2$ values is seen $(1.7-2.2 \mathrm{~ms})$, which parallels the increase in $\mathrm{pH}$ of the slurry. The proton exchange rate between the water molecules decreased with $\mathrm{pH}$, causing an increase in the proton $\mathrm{T} 2$ values. 


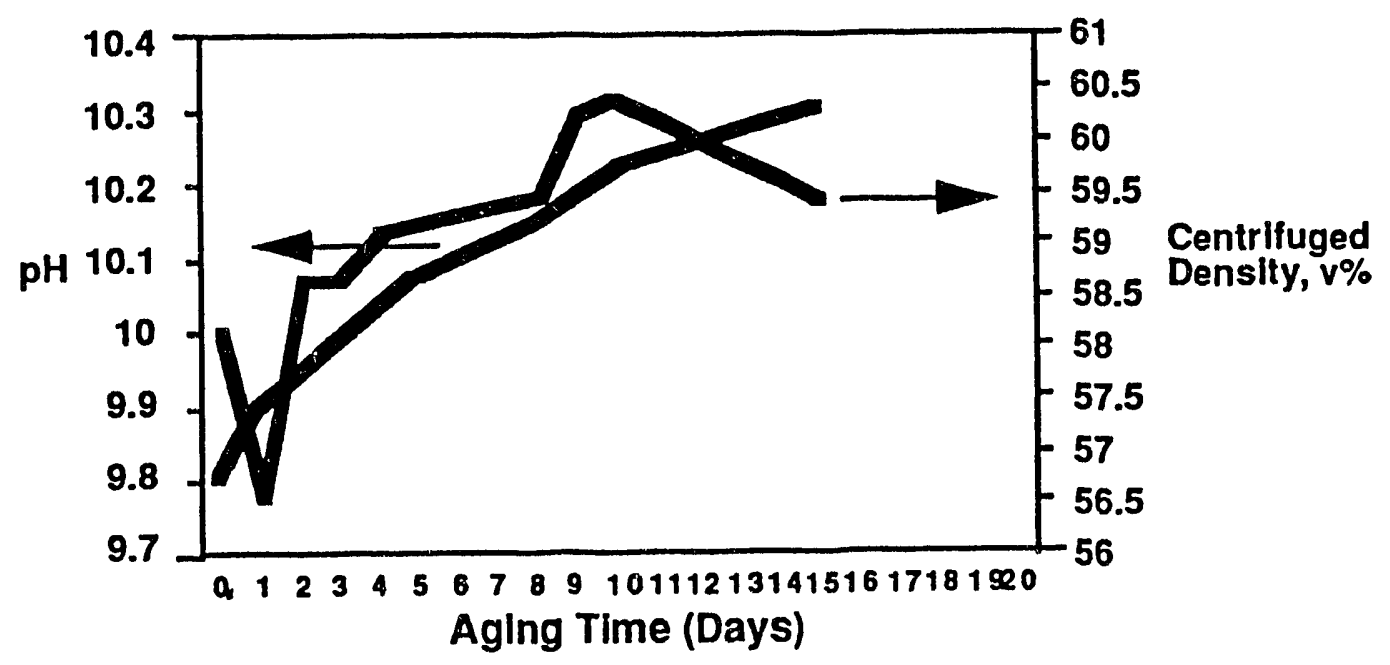

Figure 2-10: Aging Behavior of Aqueous Slurry

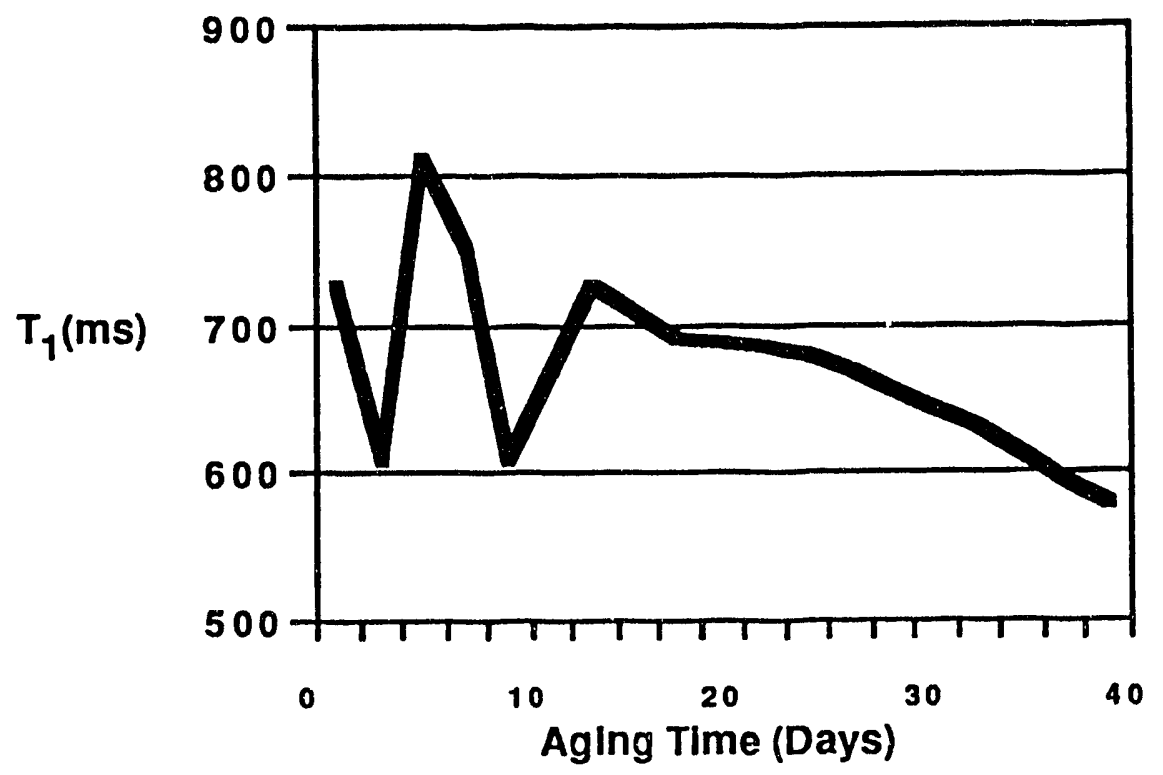

Figure 2-11: $\quad$ NMR Aging Behavior of Aqueous Slurry

The $T 1$ and $T 2$ values of pure water are about $1500 \mathrm{~ms}$. The lower $T 1$ values observed for the slurry indicate that the water molecules have restricted motion, presumably because they are tied up with the surface of colloidal silicon nitride particles. The T2 values are much shorter than $T 1$ because of chemical exchange. Reductions observed in $T 1$ inulicate more "ordering" of the water molecules as the slurry undergoes an aging process. 


\section{Mold Design}

The nature of the density variability in a cast specimen depends upon total filtration surface area and the gate location(s) of the mold. In the initial casting work the mold provided a filtration surface all around the cavity. In the modified mold design, the filtration area was reduced and at the same time, gate locations were altered. Whereas drying cracks were frequently encountered in the early work, cast bars from the modified mold showed no cracking even under uncontrolled (ambient) drying conditions. The reduced filtration surface resulted in a single casting front as opposed to multiple fronts in the full filtration surface mold. Drying related cracking occurs where several fronts meet.

A comparison of the two mold designs was conducted on a pair of pressure cast NSF bars. One of the bars was cast using 1008 filtration surface of the plaster mold, while the other had a portion of the mold surface sealed. The $10 \mathrm{~mm}$ diameter gage sections of the two specimens were imaged using $x$-ray computed tomography (CT). Four slices (each $1.06 \mathrm{~mm}$ thick) were reconstructed with a tomographic image size of $256 \mathrm{x}$ 256 pixels. The results are represented in line profiles of the image intensity across two diameters at $90^{\circ}$ to each other, Figure 2-12. The profiles display raw pixel grey level data. While smoothing would aid interpretation, there nonetheless is a significant distinguishing characteristic of the two CT scans apparent in these raw data, and it involves the intensity at the center of the profiles (bar centerline). This location is a point of low density for the 1008 filtration case, while it is a point of high density for the partial filtration case. These results suggest the possibility that the mechanism of cracking during drying of as-cast bars in the 1008 filtration case involves the low density core indicated by the data. Low core density will help generate tensile hoop stress on the surface of the bar during drying and hence contribute to the likelihood of longitudinal cracking.

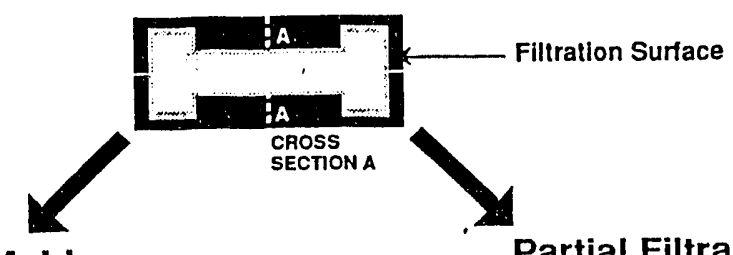

Full Filtration Mold
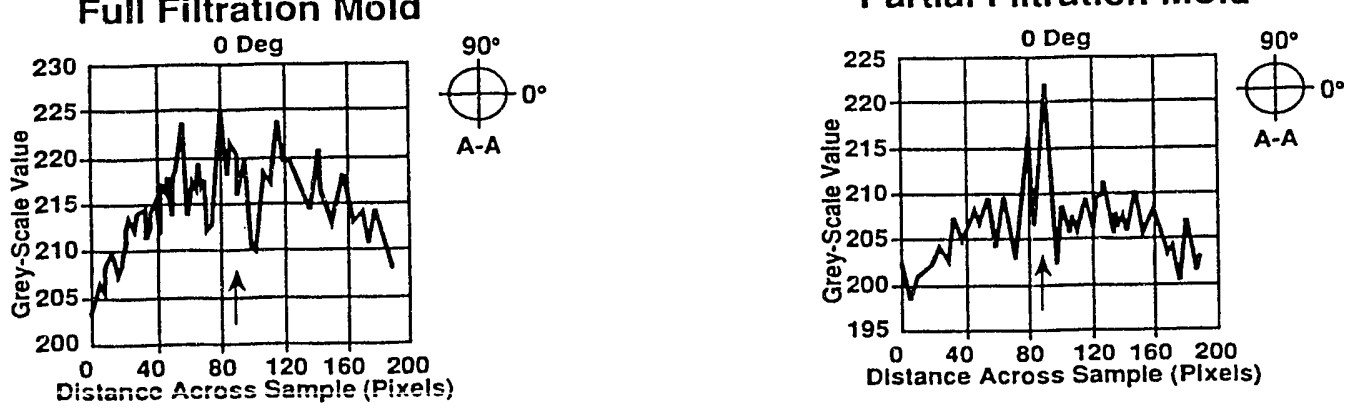

Figure 2-12:

Evaluation of Mold Design by Density Profile Measurement Using Computed Tomography 


\section{Density Uniformity Control During Filtration}

Density gradients in the cast body are generated during the pressure filtration process corresponding to permeability changes within the cast layer. A technique was developed using programmed pressure profiles to mitigate this density non-uniformity problem as described below.

Figure 2-13 illustrates the non-linear casting rate that occurs under a fixed pressure when pressure casting tensile bars. The plot was developed from cast layer thickness data during casting at constant pressure level $(0.07-0.42 \mathrm{MPa})$. The nonlinear casting rate implies an inherent density gradient in the cast cake. The cast bars from these experiments when densified showed preferential bending. By applying a programed pressure schedule during casting the cast rate can be linearized, as is approximated in Figure 2-14. Bars cast according to this schedule showed negligible distortion and thus suitably uniform green density.

Besides the linearity of the casting behavior, the casting rate magnitude also influences the level of density gradient. An SOP was developed to achieve the acceptable level of the casting rate to assure density uniformity within the specified limits. The procedure established a go/nogo criterion for acceptance of a slurry batch, as follows:

- A fixed volume of slip is cast at constant pressure on an established porous material.

- The thickness of the cast is monitored at fixed time increments.

- Thickness squared vs. time of cast is plotted for the slurry, Figure 2-15.

- The slope of the line is estimated using linear regression.

- If the slope of the line is found to be greater than specification (UCL, Figure 2-15), the slip is rejected.

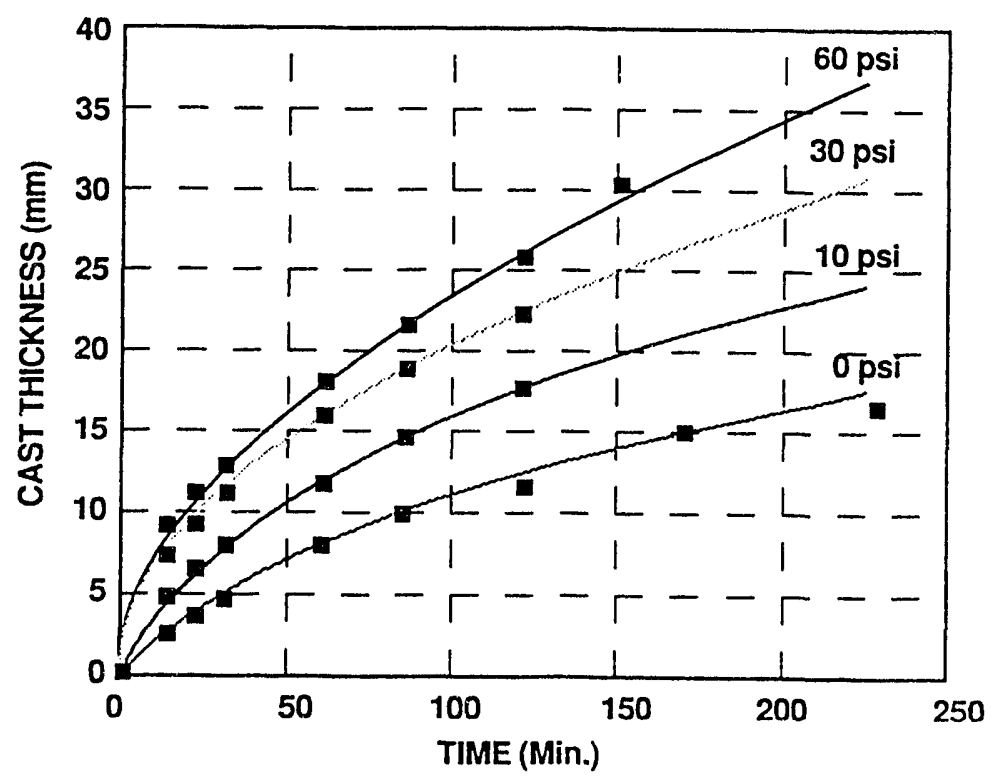

Figure 2-13:

Nonlinear Casting Rate Under Constant Pressure 


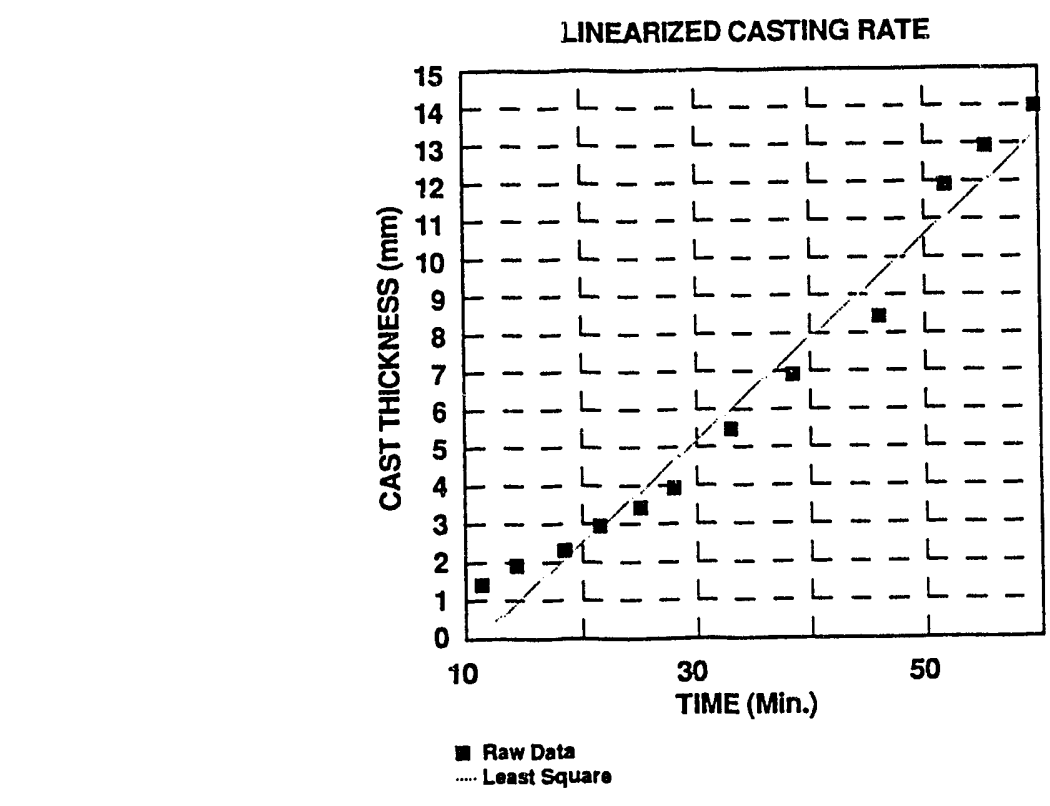

Figure 2-14:

Linearized Casting Rate with Programed Pressure schedule

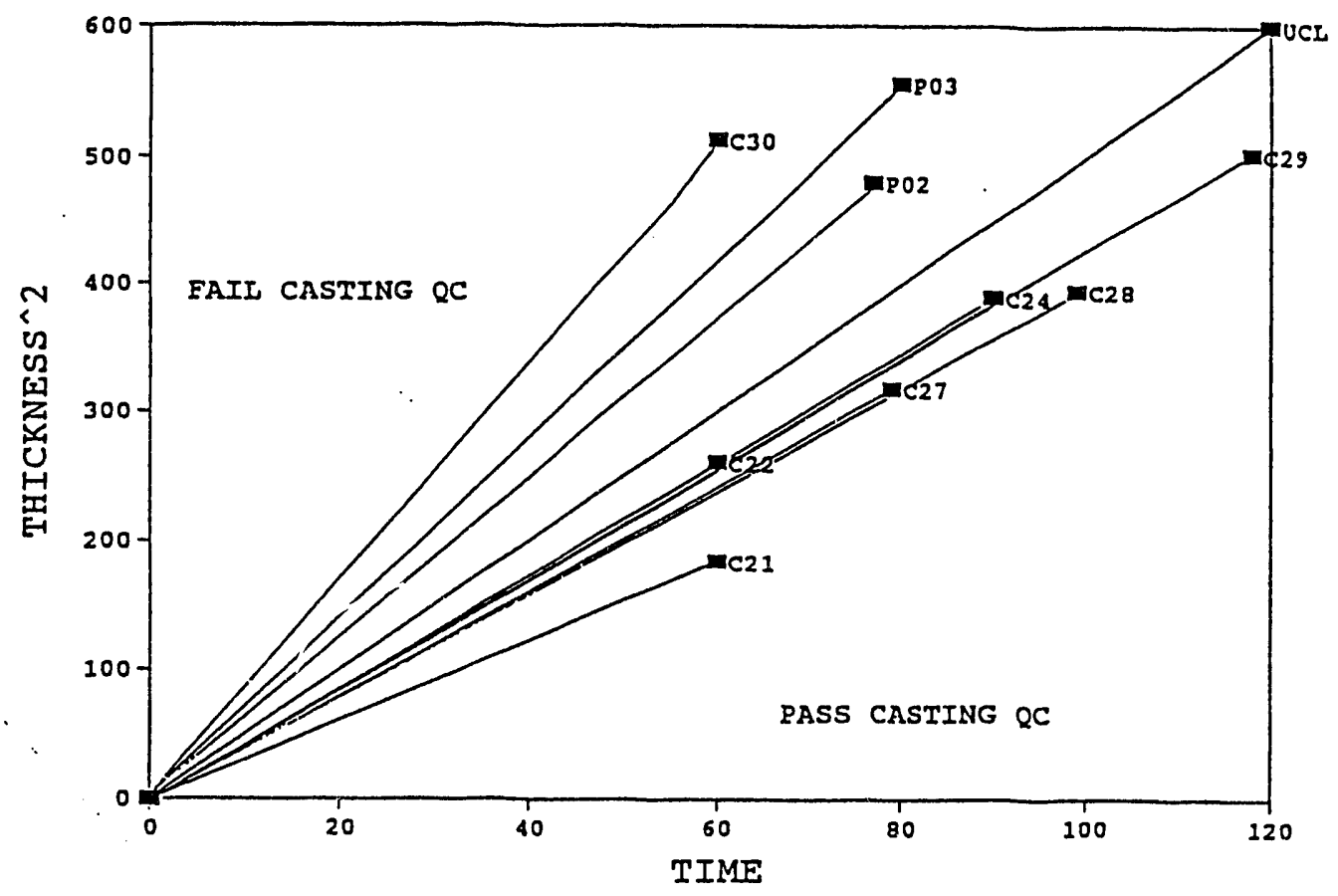

Figure 2-15: $\quad$ Puck Casting $Q \mathrm{C}$ for slurry

The importance of this casting rate measure is shown by examining the effect of the casting rate slope on the total indicated run-out (TIR), Figure 2-16. TIR is a measure of the amount of warpage or bending of the tensile rod caused by the green density gradient and determines the yield during the densification stage. Runout also has a 
bearing on the tensile strength, as it determines the amount of removal of the relatively low density reaction layer during the machining process, Figure 2-17.

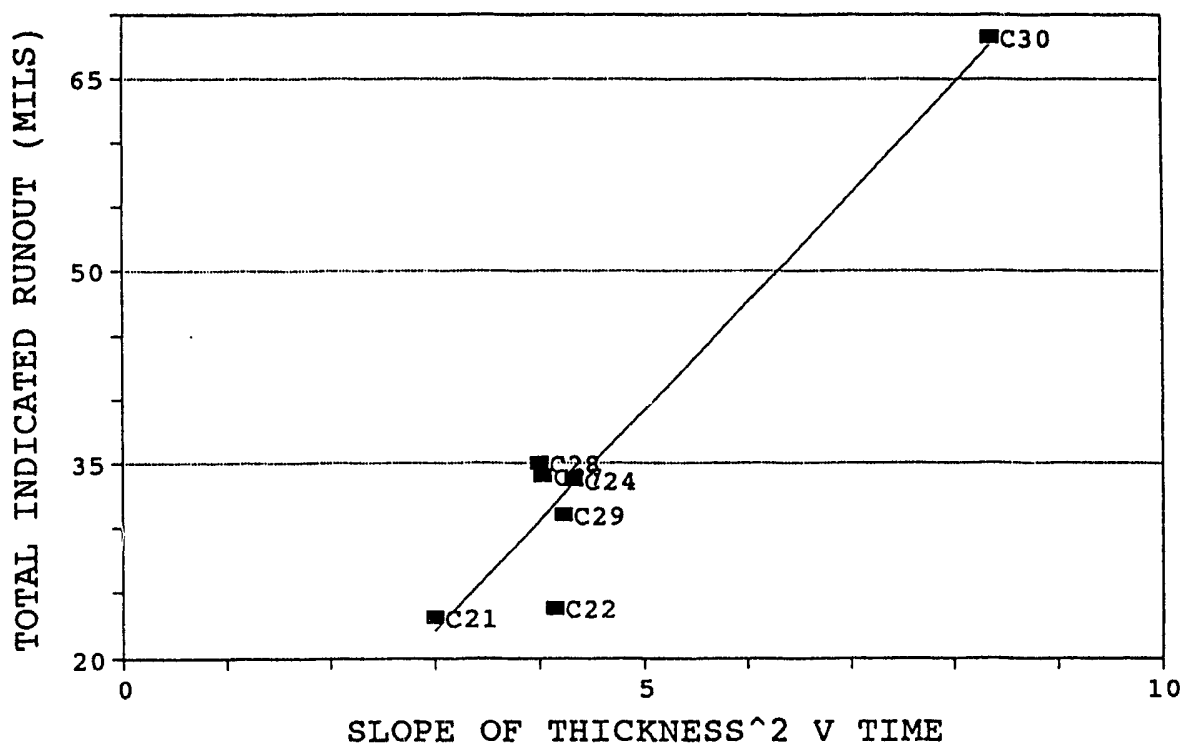

Figure 2-16: Regression of Average HIP'ed Runout on Casting Rate

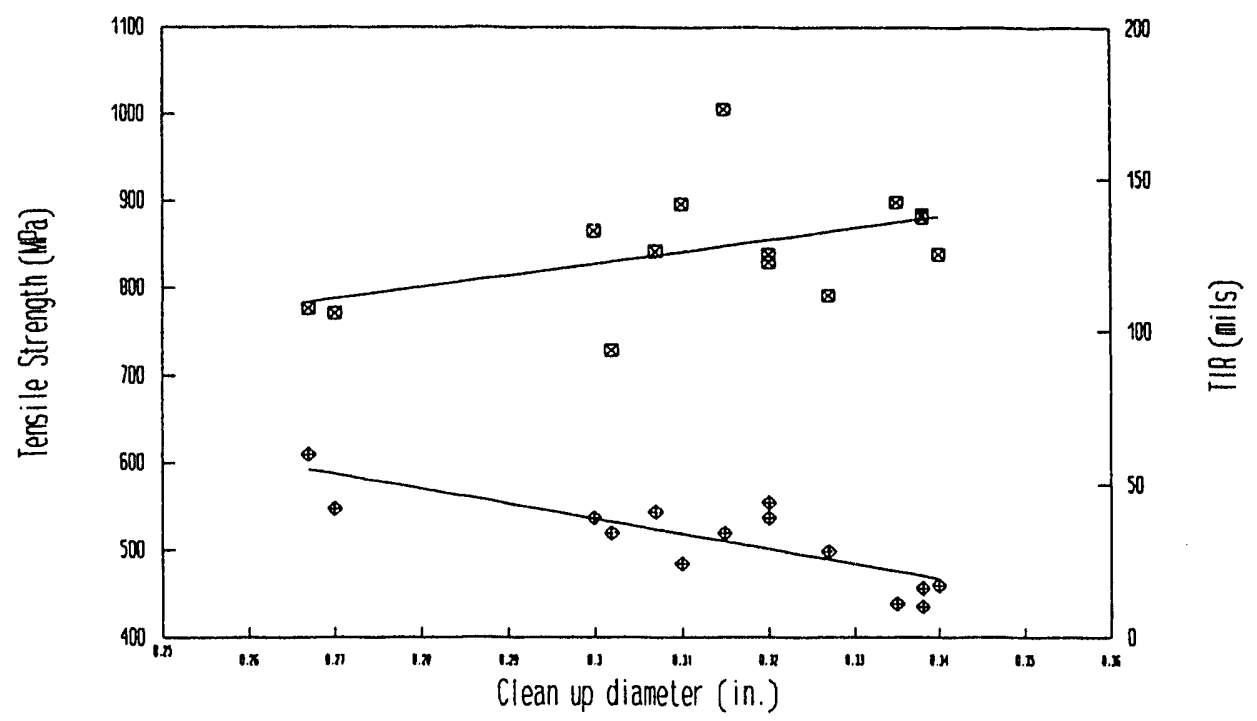

$\otimes$ tensile strength $\rightarrow$ TIR

Figure 2-17: RT Strength - Runout correlation

Casting of Net Shape Formed (NSF) Tensile Bars

In all casting experiments a well dispersed aqueous suspension of silicon nitride containing 4 w/o yttrium oxide as a sintering aid was 
utilized. The suspensions contained approximately $70 \mathrm{w} / 0$ (43v/0) solids (average particle size $0.8 \mu \mathrm{m}$ ) and were stabilized at $\mathrm{pH}=9.5$. The cast ready suspensions were characterized using a Brookfield viscometer. The suspensions showed a typical shear thinning behavior (Figure 2-18). As mentioned in the previous section, the ceramic suspensions used in this study were prepared using a closed loop aqueous comminution and slip conditioning procedure. Slip conditioning procedure included sonication followed by deairing.

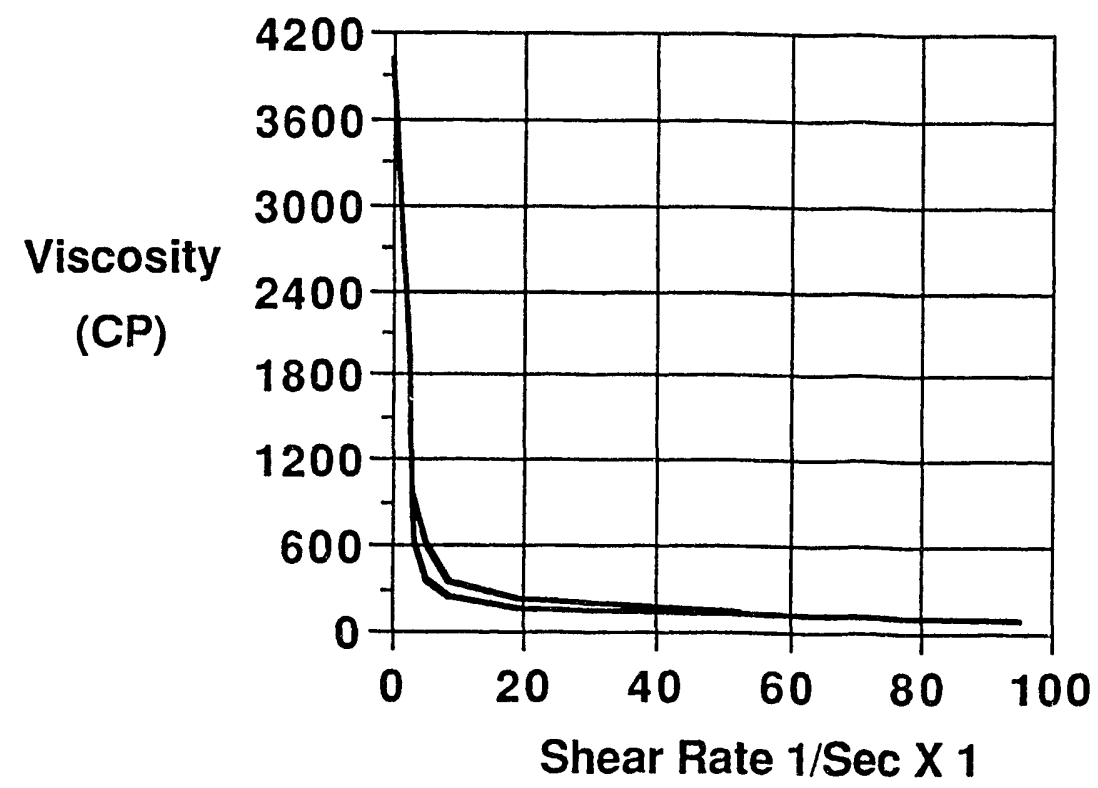

Figure 2-18:

Viscosity vs. Shear Rate for Aqueous Ceramic Suspension

Throughout the experimentation two piece plaster of Paris molds were employed having average pore size of $2.0 \mu \mathrm{m}$ (evaluated by mercury porosimetry technique). Prior to casting, all molds were preconditioned by soaking them in DI water for 3 minutes. The ceramic suspension was fed into the mold under specified pressure from the two ends of the mold cavity near the buttonhead section of the tensile bar.

Large scale NSF bar casting iterations were performed to evaluate the effects of the process variables and optimize the casting process. A program pressure profile was utilized during casting to assure linear casting rates and hence minimal density gradient. In each iteration greater than 70 NSF tensile bars were cast, dried, degassed and HIP'ed, machined, radiographed and tensile tested.

During the casting of NSF bars, some difficulty was encountered in establishing the necessary time for completion of the casting process. In some cases, overcasting resulted in shrinkage related cracking at the buttonhead radius. These forming defects are possible failure origins during tensile testing. In order to alleviate this problem, an ultrasonic (UT) based in-situ monitoring technique was developed to establish the completion time. 
Ultrasonic Monitoring of the Casting Process

Ultrasonic Characterization of the Ceramic suspension. The ultrasonic impedance of a column of ceramic suspension and a saturated cast body were established to provide baseline data needed in the process monitoring. The liquid column measured $25 \mathrm{~mm}$ diameter $\times 198 \mathrm{~mm}$ high. A $1.5 \mathrm{MHz}$ UT transducer was placed at each end of the liquid column or cast body to measure time of flight and signal amplitude (attenuation). Results are compiled in Table 2-3.

Table 2-3: Ultrasonic characteristics of casting systems

\begin{tabular}{||c|c|c||}
\hline \multirow{2}{*}{ Materials } & \multicolumn{2}{|c|}{ UT PROPERTIES } \\
\cline { 2 - 3 } & Velocity (m/sec) & Attenuation (db/mm) \\
\hline Water & 1500 & 0.0 \\
\hline $\begin{array}{c}\text { Slip } \\
(70 \text { w/o solids) }\end{array}$ & 1385 & 0.154 \\
\hline $\begin{array}{c}\text { Cast Green } \\
\text { Saturated Bar }\end{array}$ & 1516 & 0.094 \\
\hline
\end{tabular}

As expected, the attenuation in the ceramic suspension exceeds that of pure water. Sound velocity in the cast body is similar to that in water as all its pores are fully saturated. For a similar reason, the impedance in the cast body is also lower than that measured in the suspension. The ultrasonic characteristics of the slurry and the cast body established from these experiments were used to interpret the wave forms during in-situ monitoring of the casting process.

In-Situ Monitoring of the Filtration Process Casting experiments were performed in a two piece plaster mold. The mold cavity shape was that of a buttonhead tensile bar. Figure 2-19 shows a schematic of the mold cavity along with the location of the UT transducers. Two transducers (1.5 $\mathrm{MHz})$, one at each end of the mold cavity, were placed such that their active faces were flush with the end of the cast tensile bar button heads. The transducers were fixtured in the mold to assure positive contact with the cast bar throughout the experimentation and to prevent being pushed out of the mold under the applied suspension pressure. The mold cavity was fed from two gates located at the two ends of the bar near the buttonheads.

The mold cavity was pressurized with the suspension at $0.14 \mathrm{MPa}$ (20 psi) and the casting process was monitored by the attenuation of the ultrasonic signal. The ultrasonic signal was transmitted from one end and received at the other end of the mold cavity. Both the amplitude and the time of flight were monitored throughout the experimentation using a personal computer, $\bar{F}$ igure $2-19$. A coinputer aigorithm was designed to acquire maximum signal amplitude and the corresponding time of flight at regular time intervals. 


\section{Through transmission}

- a. Amplitude

-b. Time of flight

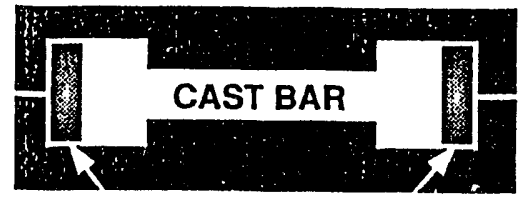

UT TRANSDUCER

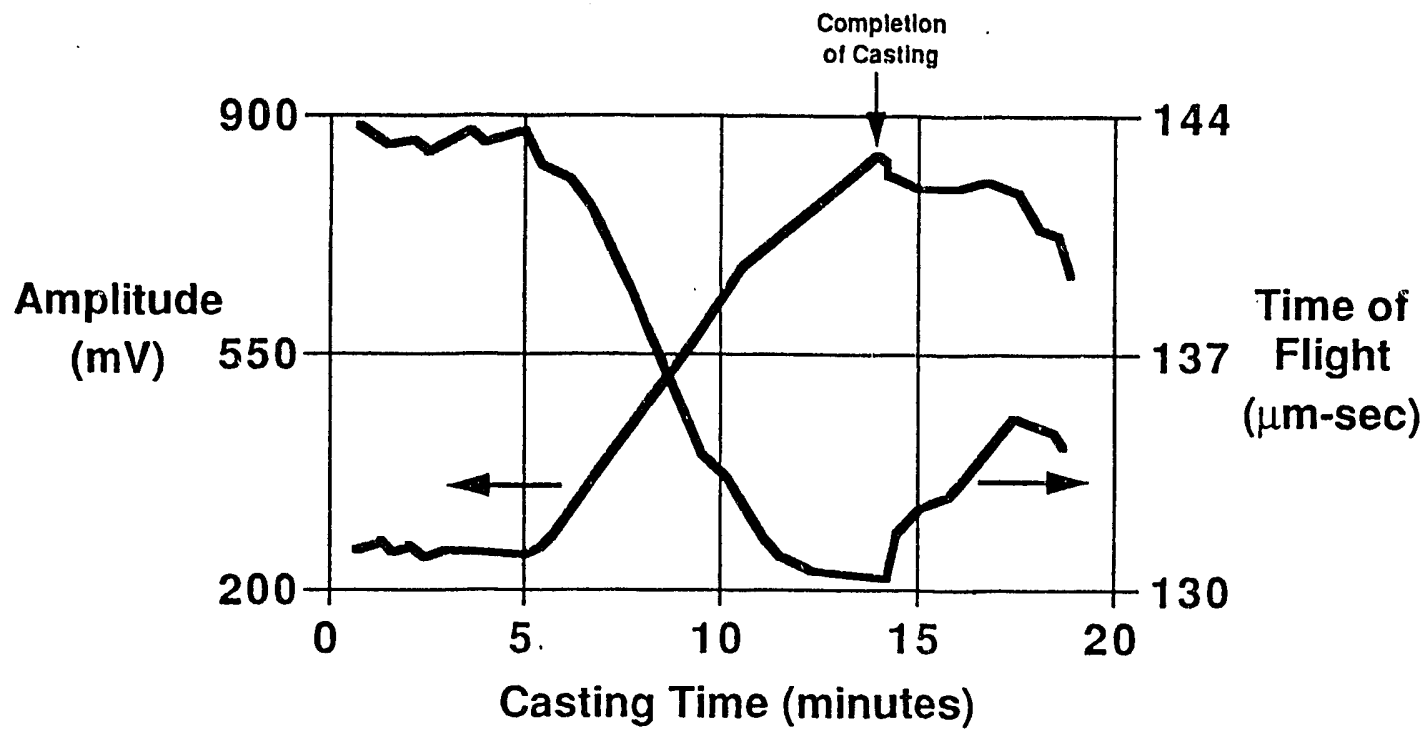

Figure 2-19: تiltrasonic In-Situ Monitoring of Tensile Bar Casting

As suggested by Figure 2-19, as the cast layer thickness increases, the received signal amplitude increases while the time of flight decreases. This trend continues until the completion of the casting process. Upon completion the amplitude and the time of flight achieve their highest and lowest values respectively. This may be explained by the fact that at this point the cavity is completely filled with the powder and the interstitial spaces are fully saturated with the liquid (water), thus providing highest conductance path for the UT signal. Beyond this point, the suspension supply to the cavity ceases due to the casting of the gate. However, because of the continued drainage of the pore fluid through the paster mold, water content in the cast body decreases causing precipitous drop in the UT signal amplitude. At this point the cast body should be removed from the mold. If, on the other hand, the drainage process is allowed to continue (overcasting condition), the cast part begins to shrink non-uniformly inside the nold resulting in undesirable cracking, specifically in the transition regions (as would be the case for a turbocharger rotor casting). Typical wave forms of the received signal as a function of the casting time are shown in Figure 2-20. In the beginning $\left(t=t_{1}\right)$ the signal is transmitted through the suspension only and a single wave signature appears on the oscilloscope screen. As the casting progresses ( $t=t 2$ ), the signal is transinitted both through the suspension and the cast layer and two separate signatures appear. With further progress in casting ( $\left.t=t_{3}, t_{4}\right)$, more and more signal is carried through the cast 
layer and hence the cast layer signature amplitude eventually exceeds that of the suspension. With increasing signal amplitude the correstonding time of flight decreases. Finally, the suspension signzture disappears $(t=t 5)$ leaving a single signature representing the crist body.

\section{Signal}

\section{Amplitude}

Figure 2-20:

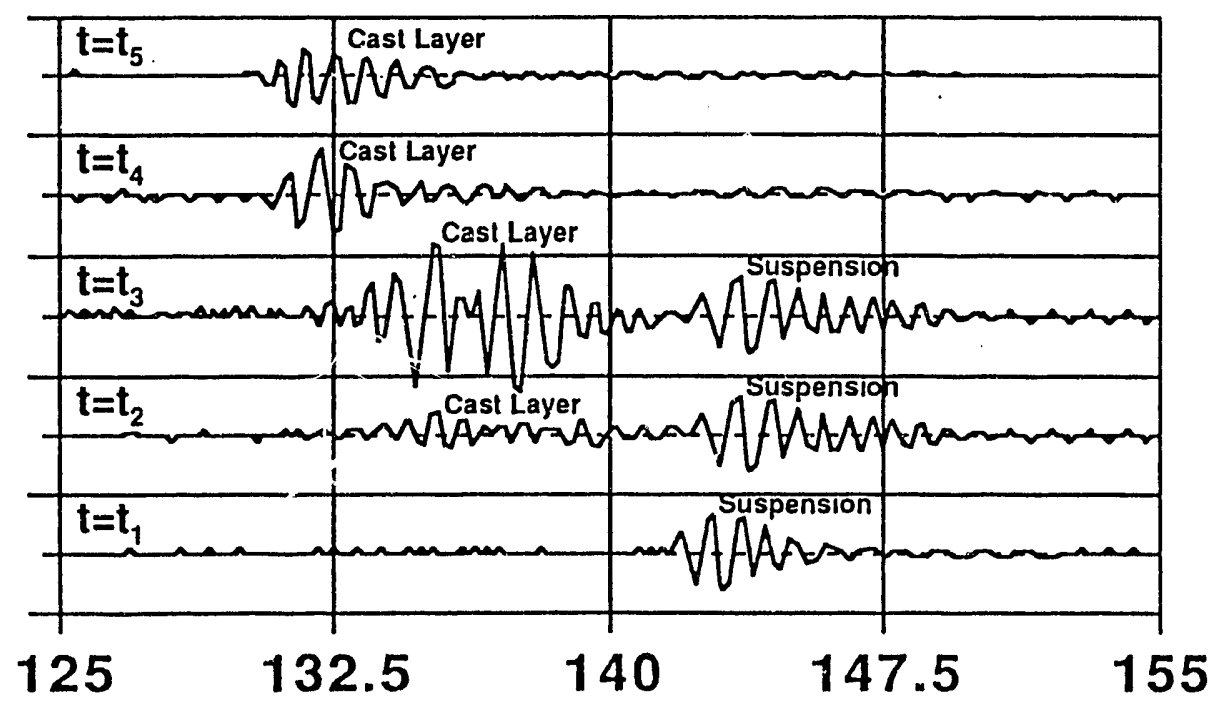

\section{Time of Flight ( $\mu$-seconds)}

UT signal characteristics as a Function of casting Time

The results have been reproduced for various applied pressures and suspension concentrations thus providing a simple and yet effective way to monitor and control the casting process.

Comparison of Ultrasonic and Physical Casting Rate Data. Cast thickness physical measurement data are plotted in Figure 2-21 with the UT amplitude data from Figure 2-19. These data correspond to the casting experiments performed ai a fixed applied pressure of $0.14 \mathrm{MPa}$ (2) psi). The casting kinetics data from physical measurements are in reasonably good agreement with the UT data suggesting that the UT amplitude could be utilized as a feedback signal to adjust the applied pressure and hence control the casting rate for an intelligent processing methodology.

\section{INJECTION MOLDING PROCESS DEVELOPMENT}

Injection molding was the initial process in the program and supplied baseline tensile strength data. With reference to Figure 2-4, the process sieps subsequent to siurry concentration were as follows: freeze drying, compounding, injection molding, de-waxing, pre-sintering (de-gas) and HIP'ing. Here compounding, mold design, molding process optimization, ant dewaxing will be addressed. 


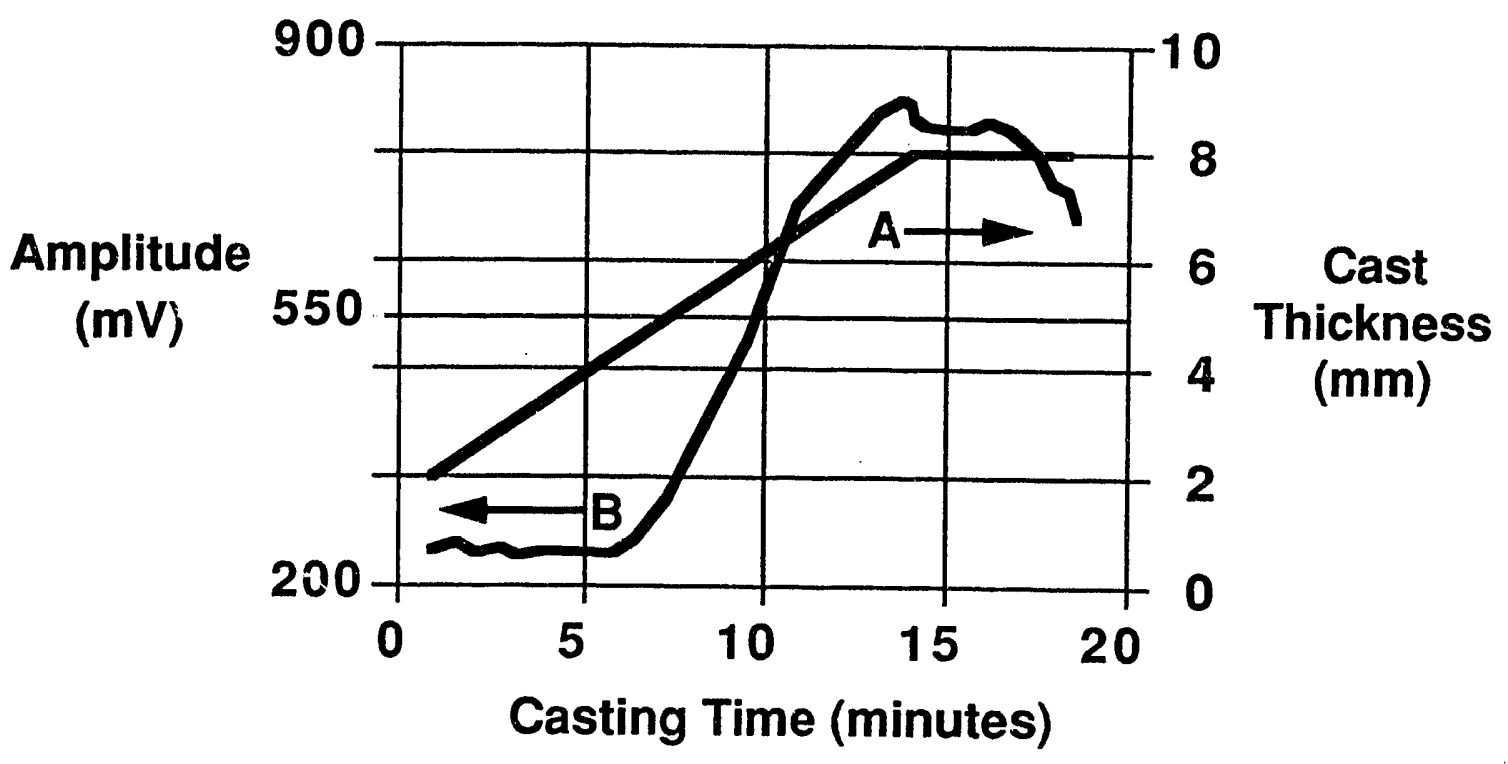

Figure 2-21: Comparison of Casting Rate Prediction from Ultrasonic (B) and Physical Measurement (A)

Compounding

The molding compound was prepared by mixing the powder with a thermoplastic based binder system under high shear. Molding compound was fully characterized by measuring the rheology (viscosity as a function of shear rate and temperature), thermal conductivity, P-V-T (pressure-volume-temperature) correlation, density and specific heat, as described below.

\section{Mold vesign}

In order to optimize the mold lesign, two techniques were used. The first was experimental includi, experimental design and analysis of the molded parts using both desc...tive and nondestructive methods. A second task was conducted in parallel to the experimental work which involved finite element modeling (FEM) of the mold filling process. The FEM modeling work was conducted in collaboration with CAE Technology Inc., Ithaca, New York. Two different gate designs were evaluated experimentally which are designated as end gate, "G2", and fan gate, "G3" as shown in Figure 2-22. The experimental work provided both information on initial conditions necessary for the FEM work, and later, confirmation of the results generated by the modeling.

The experimental work was carried out using a mold with a single tensile bar cavity. Factorial experimental designs were utilized in order to evaluate both mold design as well as molding parameters. All tensile bar specimens molded with various combinations of molding parameters were fully characterized by microfocus X-ray for internal voids. The NDE data from the two gate designs suggested little difference between the two designs regarding void density. 


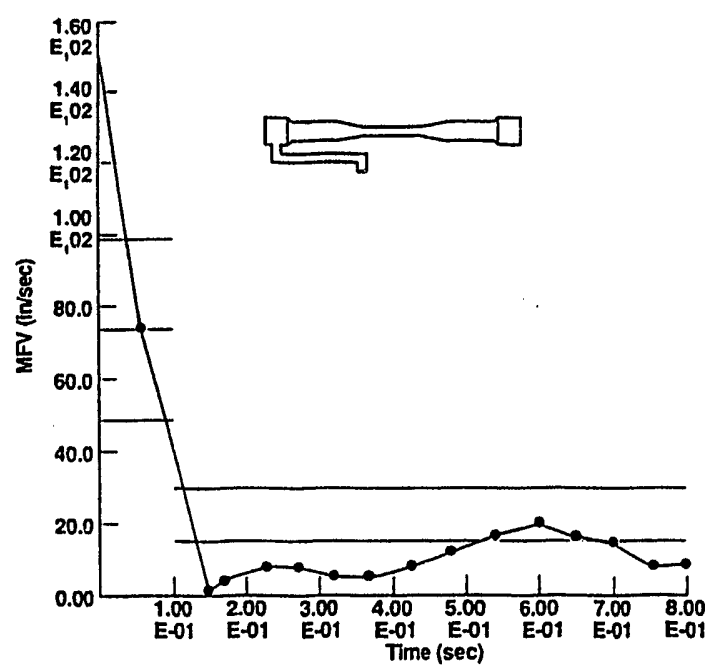

Single Flow Front For End Gate

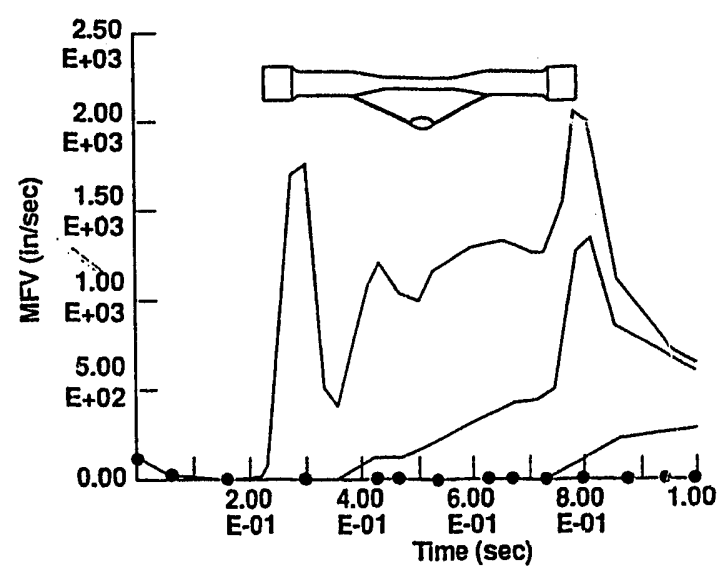

Multiple Flow Fronts For Fan Gate

Figure 2-22: Injection Molding Melt Flow Velocity by FEM

The finite element modeling required two steps: materials characterization and mold filling simulation. The materials characterization determined the density, specific heat, thermal conductivity, rheology and pressure-volume-temperature behavior of the ceramic molding compound. The rheological behavior is plotted in Figure 2-23. Table 2-4 summarizes the remaining thermal and physical properties of the material.

The material properties, along with the initial molding parameters determined experimentally, were used to conduct the computer simulations for mold filling and packing. The two gate designs, G2 and G3, were modeled in varying sizes. Preliminary analysis indicated that the end gate should give better results based on a more uniform distribution of shear rates during filling of the part. The fan gate showed a tendency for the gate to freeze off in certain areas, resulting in a split flow of material, resulting in the possibility for air entrapment during the filling. Further analysis confirmed that the end gate, with the proper speed profile, would yield a nearly consistent (single) melt front velocity (Figure 2-22) as compared to multiple melt fronts for the fan gate design. Multiple flow fronts could result in fold lines which could serve as internal crack sites after dewaxing.

Molding Process Optimization and Control

A Taguchi-based experimental design was employed to optimize the molding parameters. Ail moided parts were examined by mierofocus $x-r a y$ to establish frequency and size of molding related voids. From this study, injection pressure and barrel temperature profiles were found to be the significant process parameters. Total void volume in the tensile 
bar is plotted as a function of the two molding parameters in Figure 224. This contour plot indicates that the optimum (minimum void volume) condition is achieved at intermediate temperature and pressure profiles.

Table 2-4: Ceramic Molding Compound Material Property Data

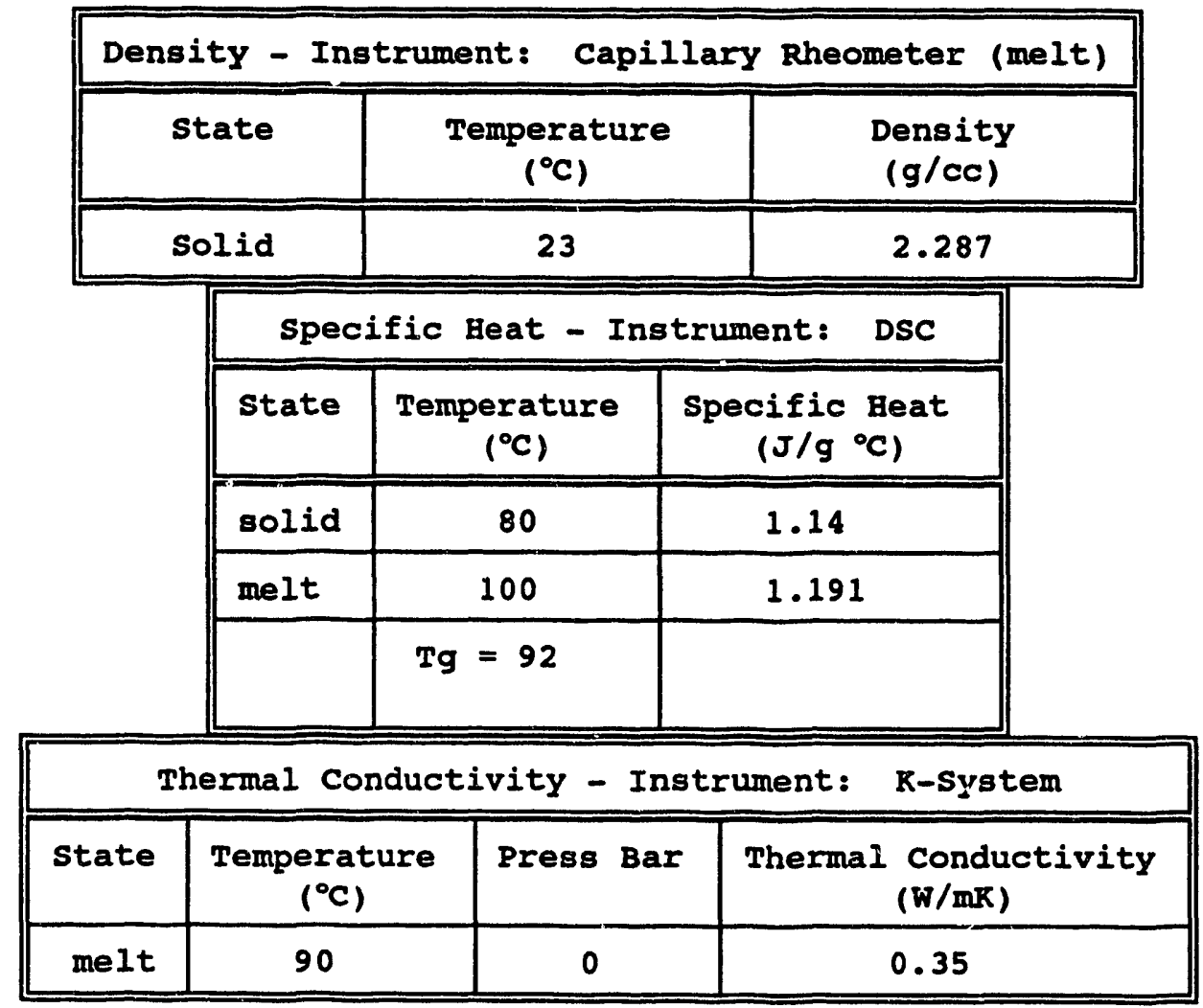

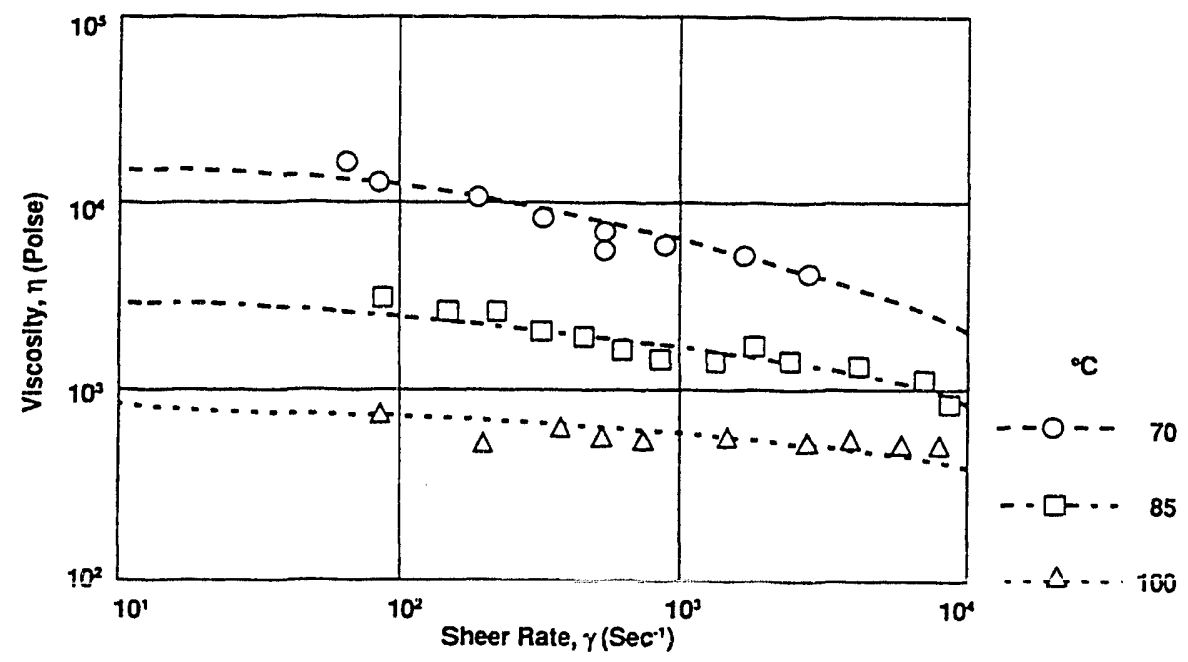

Figure 2-23: Rheological Properties of Injection Molding Compound 


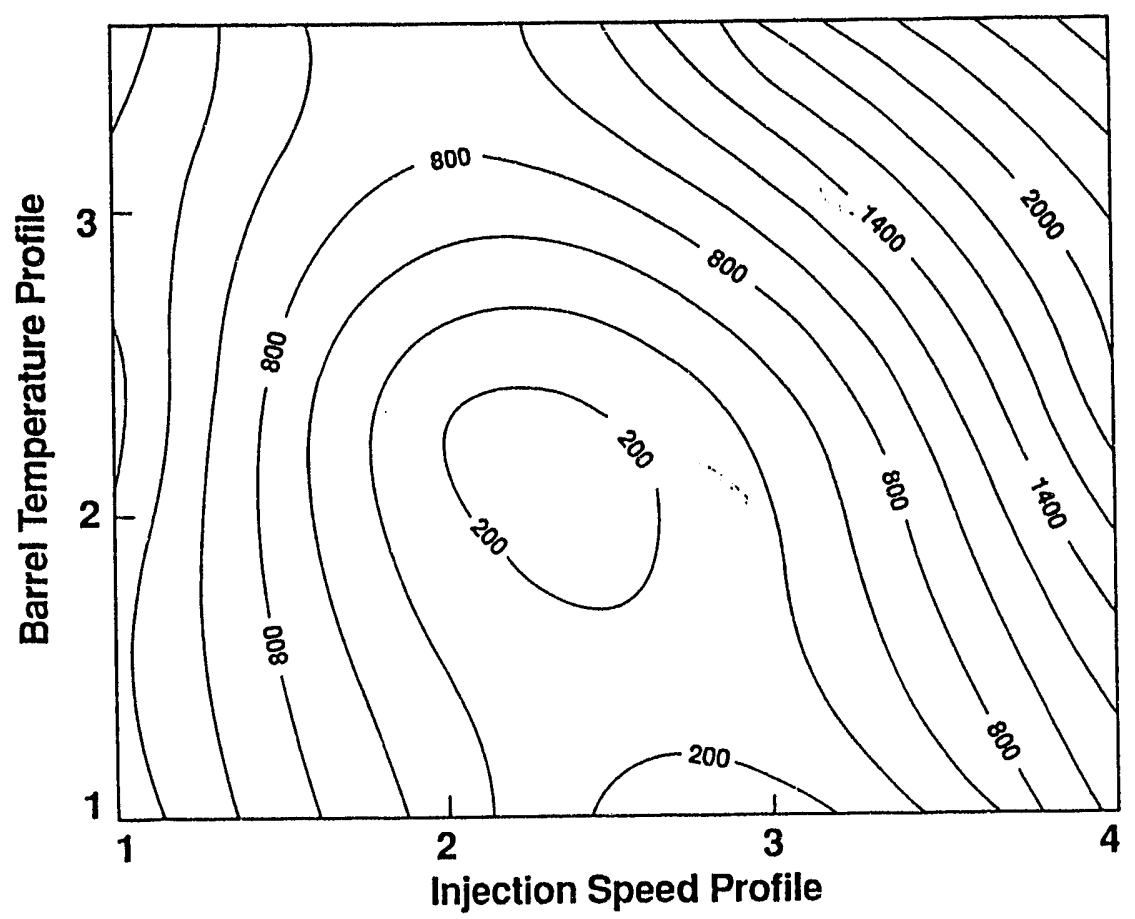

Figure 2-24: Radiograph Void Volume as Function of Molding Parameters

Approximately 240 tensile bars were molded in a two cavity mold, using the optimum molding conditions described above. since the two cavities are symmetrically located inside the mold, the kinetics of mold filling for each cavity follc's that observed in the single cavity mold. All bars were weighed, measured and examined by microfocus $X$-ray. The variation in weights and lengths was found to be 0.28 and 0.38 , respectively.

Microfocus X-ray examination results were also used to develop a flaw population histogram describing soundness of tensile bars. Figure 2-25 shows total number of flaws (voids and inclusions) observed in all 240 tensile bars at various regions of the bars. An important point to notice here is that the buttonhead farthest away from the gate shows higher frequency of flaws possibly due to incomplete mold filling. The high frequency of flaws observed in the gage section may reflect the enhanced resolution there due to smaller cross-section. The flaws detected in the as-molded tensile bars ranged between 100-300 $\mathrm{mm}$.

A good correlation was found between the weights of molded rods and the void volume detected by NDE, as shown in Figure 2-26. This suggests that the weights could be used as an on-line control variable to monitor the process. The total void volume is a summation of individual void volumes, assuming each void to be spherically shaped.

\section{Dewaxing}

The dewaxing step involved heating of the molded bars in an inert environment at a prescribed schedule. Inert environment was found to be superior to the vacuum or oxidizing condition. In the inert 
environment, the decomposition rate of the binder is suppressed which minimizes the blistering problem.

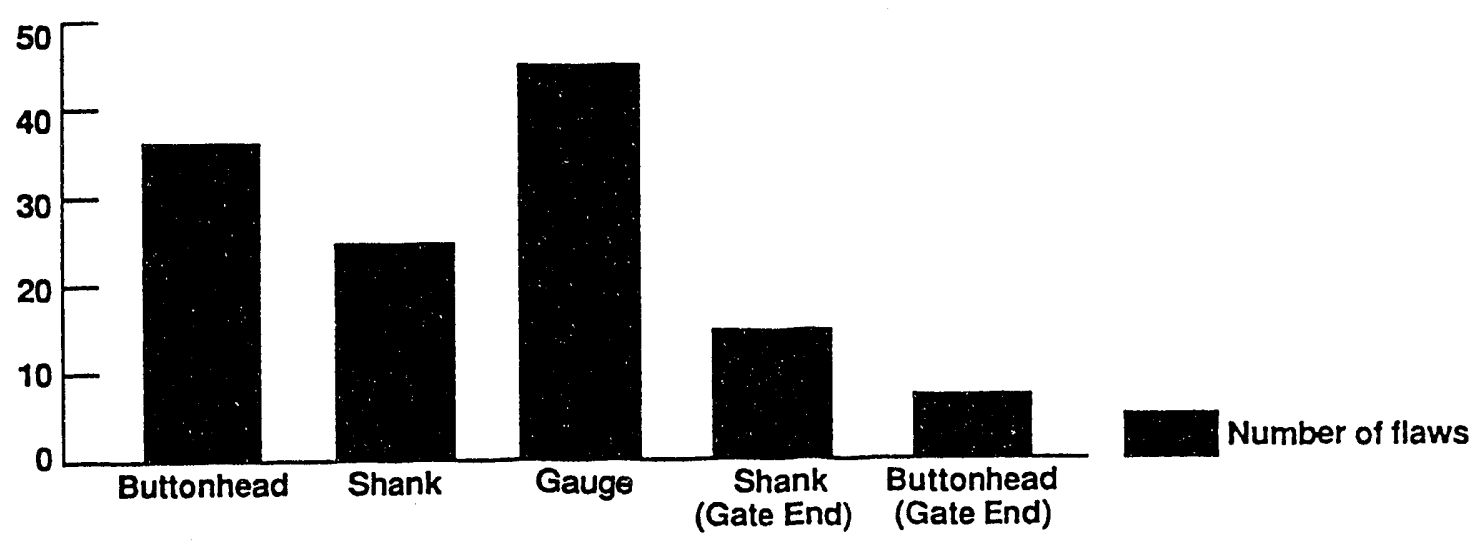

Figure 2-25: Total Flaw Distribution in 240 As-Molded Tensile Bars

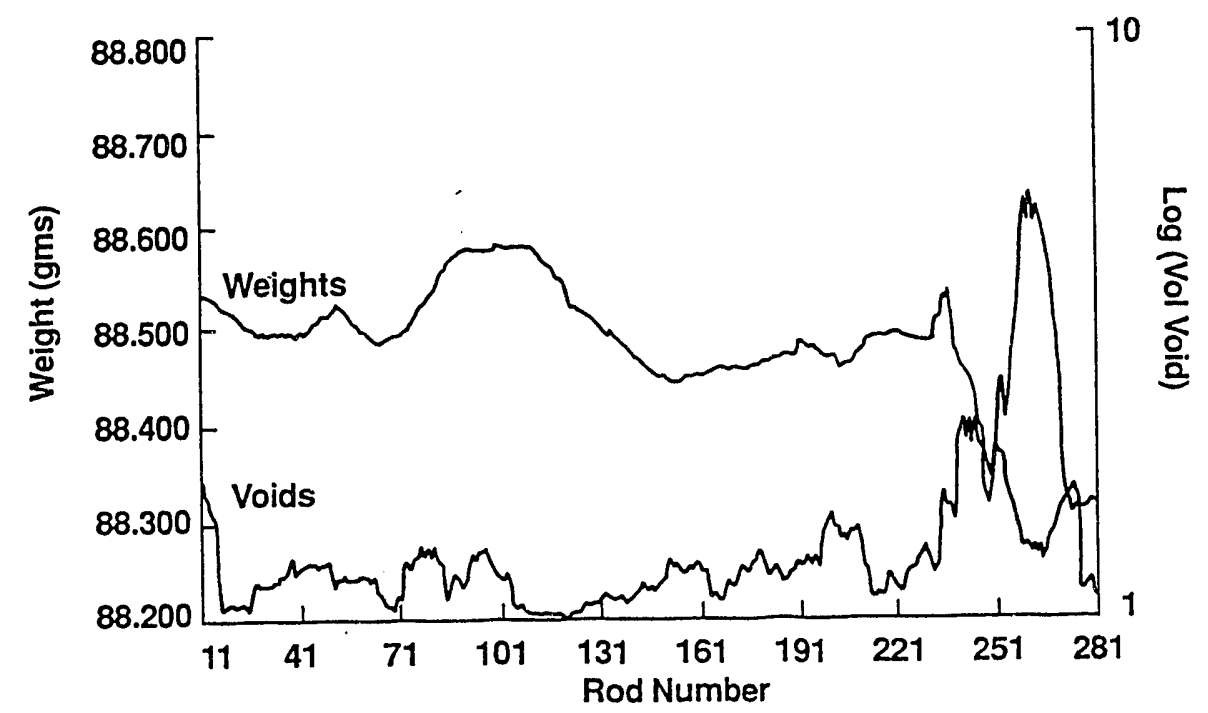

Figure 2-26: Part Weight Correlated to Radiographic Void Volume

HOT ISOSTATIC PRESSING

This section summarizes the HIP process and microstructural development. Comparing the effect of powder processing and forming modifications on strength and strength distribution required the reproducibility of the HIP cycles. Uniform densification was important to eliminate this operation as a source of strength variability. Based on the results of an experimental matrix, the HIP cycle was modified to optimize the toughness obtained in the tensile rods.

In addition to propertỹ uniformity, hif loading was maximized to minimize the number of HIP runs required for production of Stage III tensile rods. Overall HIP yield in the vicinity of 80-858 was achieved in the program. 
HIP Process Development

During stage I and through part of stage II of this program, HIP loading procedures included the use of fixtures to support the tensile rods. The procedure resulted in low loading capacity and poor yields. The HIP capacity with an alternative loading/fixturing procedure was increased from 15 tensile rods to a capacity of 30 tensile rods. The HIP procedures with standard and alternate loading were compared using tensile rods from the same batch. The improved yield and density of the alternate approach are seen in Table 2-5.

Table 2-5: Comparison of HIP Procedures

\begin{tabular}{|c|c|c|c|c|}
\hline Mill Batch & Cast Batch & Loading & Density $(\mathrm{g} / \mathrm{CC})$ & Yield(8) \\
\hline \hline $\mathrm{C} 013$ & $\mathrm{~N}$ & Standard & 3.178 & 43 \\
\hline $\mathrm{C} 013$ & $\mathrm{~N}$ & Alternate & 3.211 & 86 \\
\hline
\end{tabular}

Four HIP runs verified the alternative HIP procedures and the results are summarized in Table $2-6$. The overall yield of 868 demonstrates the viability of these procedures. The criteria for an acceptable tensile rod require an unbroken component with at least 99.58 theoretical density. The process yield control chart in Figure 2-27 shows that the HIP procedure is reproducible. The low yield in the July 12 th HIP run was caused by a thermocouple failure. The overall HIP process yield is approximately 83 percent. These procedures were followed as SOP for the remainder of the program. Further HIP process yield improvements would be feasible through fine tuning of the HIP process and better green microstructure uniformity.

Table 2-6: Summary of HIP Results

\begin{tabular}{|c|c|c|c|}
\hline Mill Batch & Cast Batch & Density(g/Cc) & Yield(8) \\
\hline \hline $\mathrm{C} 013$ & $\mathrm{~N}$ & 3.211 & 86 \\
\hline $\mathrm{C} 014$ & $\mathrm{O}$ & 3.219 & 100 \\
\hline $\mathrm{C} 014$ & $\mathrm{O}$ & 3.219 & 66 \\
\hline $\mathrm{C} 015$ & $\mathrm{P}$ & 3.211 & 89 \\
\hline
\end{tabular}




\section{Microstructural Development}

The fracture toughness of NCX-5102 is a critical factor in determining the tensile strength of the material. In stage II the fracture toughness variability was dependent on the reproducibility of the BIP cycle. The fracture toughness variation for Batch $v$ is shown in Table 2-7. The three AIP cycles of Table 2-7 had identical temperature and pressure profiles.

The HIP silicon nitride grain size, toughness and strength dependence on densification temperature, time and pressure were studied in a matrix of experiments. The following conclusions may be drawn from these sets of experiments. Temperature has a very significant effect on toughness. Time at temperature also has a significant effect on toughness. The level average toughness for temperature and time are plotted in Figure 2-28. The effect of temperature and time are significant at a 95 percent confidence level. The mechanical property trends were correlated with the final microstructures. Two measurements were made to describe the microstructure. First, the grain thickness was estimated from $5000 \mathrm{x}$ micrographs of the etched microstructure. The thickness of hexagonal or rectangular shaped grain cross gections that intersected lines drawn across the micrographs were recorded. The resulting thickness distributions should approximate the actual $B$ silicon nitride thickness distribution. Figure $2-29$ is a lognormal plot of the grain distributions at $1840^{\circ} \mathrm{C}$ for various soak times. As is obvious from Figure 2-29, grain coarsening occurs at $1840^{\circ} \mathrm{C}$ with time. Along with the increasing average grain size, the frequency of fine beta grains decreases.

The second measurement attempted to characterize the grain aspect ratio. The length and thickness of rectangular grains that intersected lines drawn across $2500 \mathrm{x}$ micrographs were tabulated. The distribution of aspect ratio as a function of grain thickness is shown in Figure 230. The aspect ratio increases with decreasing thickness. The minimum aspect ratio appears to be 4 for any grain thickness. The high aspect ratio grains tend to be associated with grain thicknesses $<0.6 \mu \mathrm{m}$.

An additional microstructural parameter, namely, aspect frequency, was also computed. The aspect frequency is the number of grains measured with an aspect ratio greater than 4 divided by the total lineal distance of the lines drawn across the micrographs. This is somewhat arbitrary, but has a basis in toughening theory and microstructural observations of the HIP silicon nitride. The main toughening mechanism in HIP silicon nitride is assumed to be crack deflection. Deflection models predict toughness is dependent on volume fraction of rods ${ }^{1}$. The aspect frequency is considered analogous to the volume fraction of rods. 


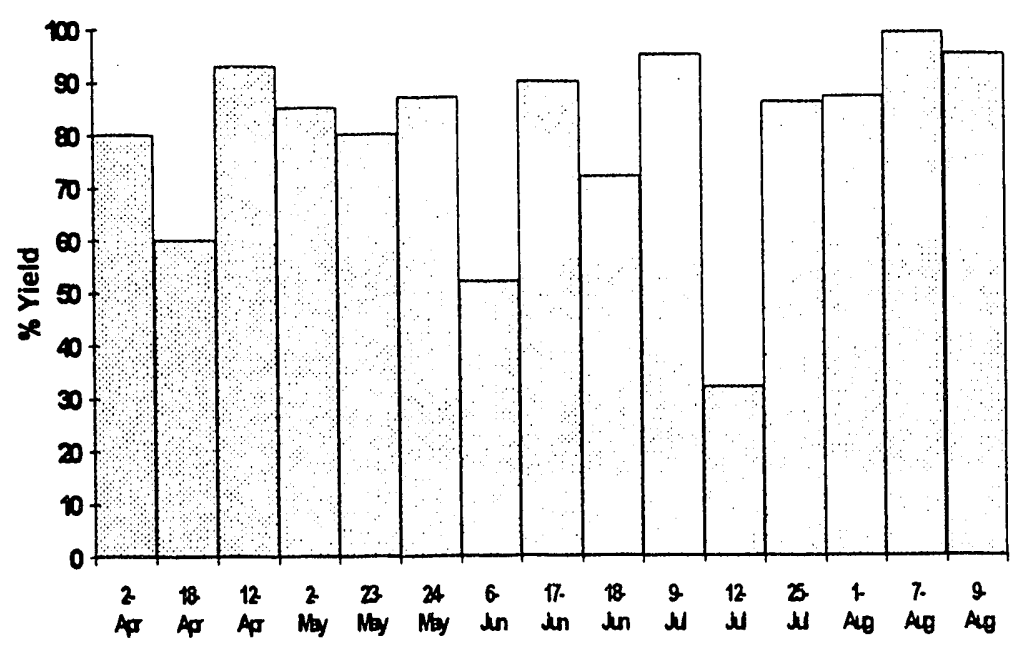

Figure 2-27:

HIP yield during stage 2 using the HIP loading procedures without fixtures.

Table 2-7: Toughness Variability observed with Batch $v$

\begin{tabular}{||l|c|}
\hline BIP Cycle & $\begin{array}{l}\text { Fracture Toughness } \\
\left(\mathrm{MPa} \mathrm{m} \mathrm{m}^{1 / 2}\right)\end{array}$ \\
\hline \hline August 1 & 6.51 \\
\hline August 7 & 5.85 \\
\hline August 9 & 5.73 \\
\hline
\end{tabular}

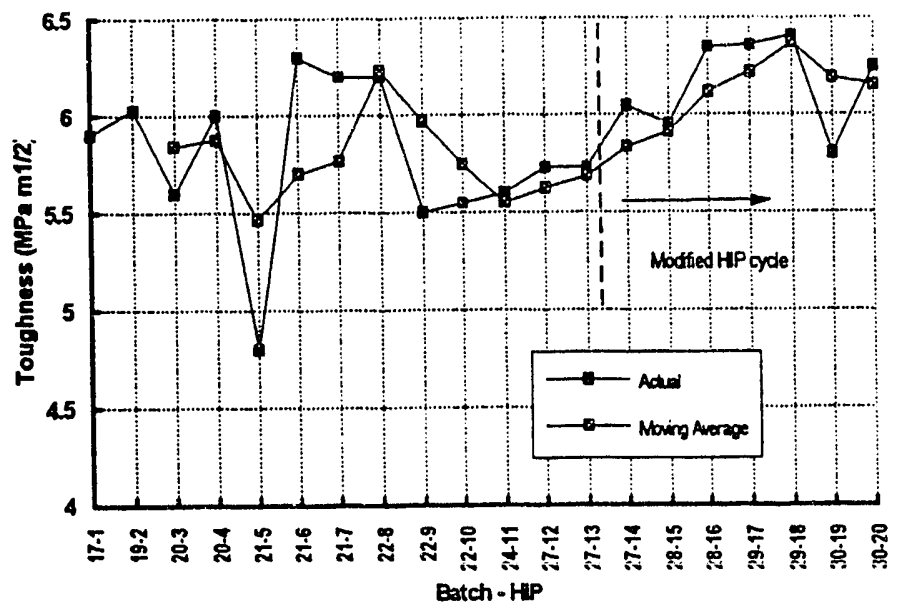

Figure 2-28: Toughness Moving Average for stage 2 


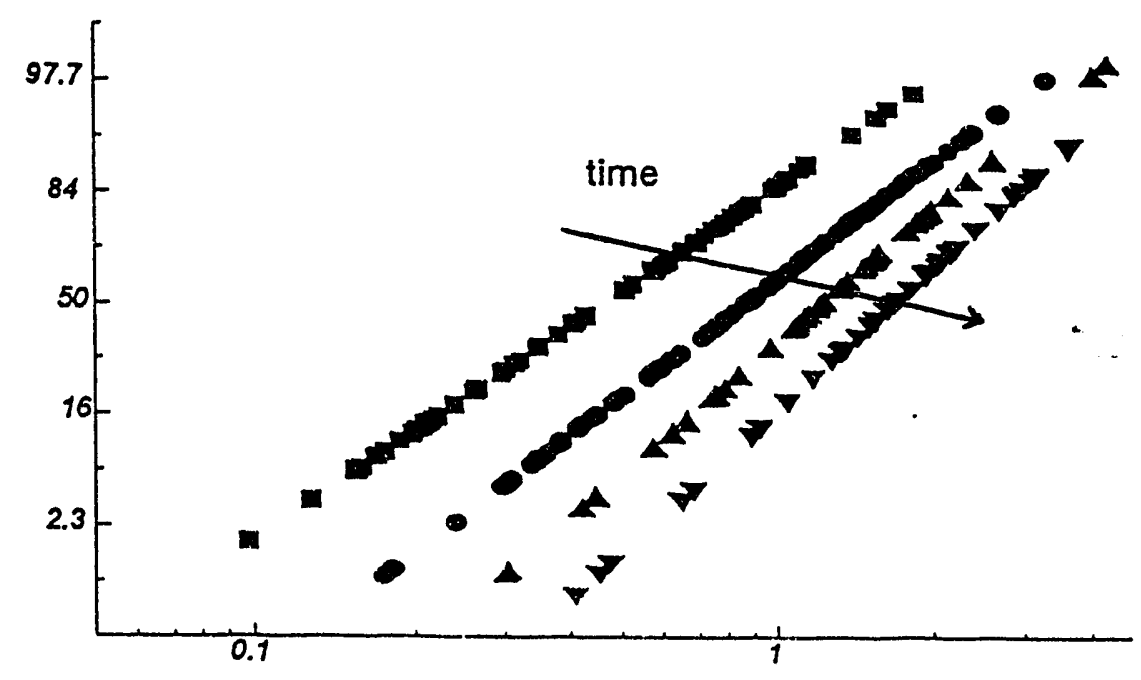

Grain Thickness (microns)

Figure 2-29: Lognormal Plot of B Silicon Nitride Grain Thickness Distributions After HIP at $1840^{\circ} \mathrm{C}$ for Different Soak Times.

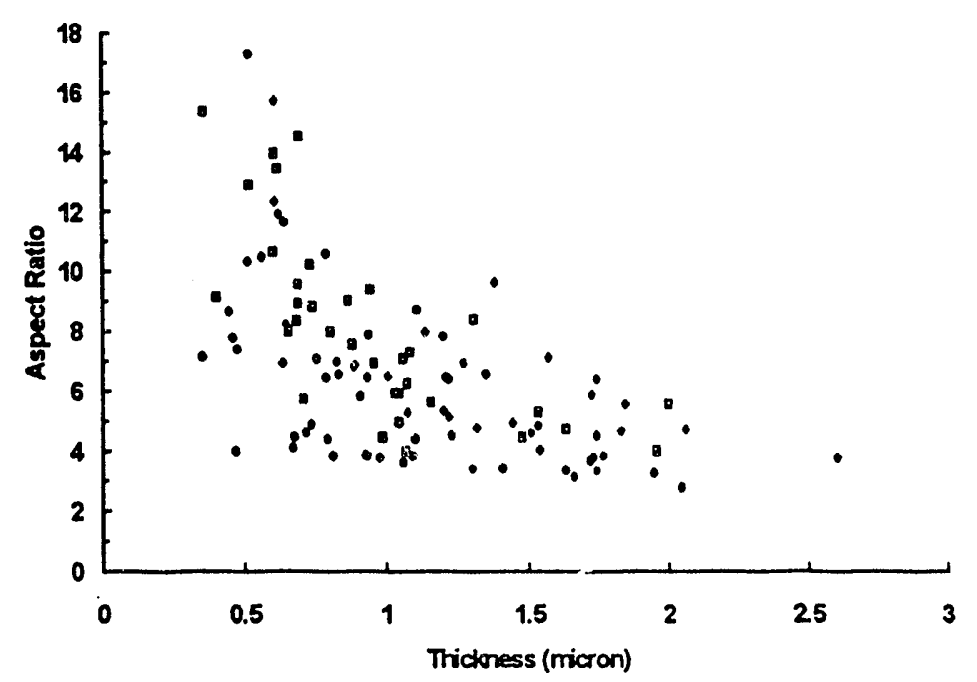

Figure 2-30:

Plot of Aspect Ratio vs. Grain Thickness

An ANOVA analysis of fracture toughness included grain thickness, aspect ratio, and aspect frequency as factors. The strongest correlation existed betweeñ toüghness âna the aspect frequienchy, Figure 2-31. The correlation coefficient was measured to be 0.97 . At an aspect frequency of 0.0 the toughness is approximately $5.12 \mathrm{MPa} \sqrt{\mathrm{m}}$. Because the aspect frequency counts only those grains with an aspect 
ratio above 4 , these results indicate that a toughness of $5.12 \mathrm{MPa} \sqrt{\mathrm{m}}$ is expected with an aspect ratio of 4 . Stronger correlation between and aspect frequency as compared to the grain thickness indicates that the principle toughening mechanism is crack deflection in HIP silicon nitride with 48 yttria. In the deflection models by Faber and Evans ${ }^{1}$ the toughness increase was dependent on the volume fraction of fibers and fiber aspect ratio. The fiber diameter was not a factor in the crack deflection model. An example of the microstructure difference between high and low toughness materials is shown in Figure 2-32. Higher frequency of high aspect ratio grains for high toughness HIP condition as compared to low toughness HIP condition is obvious from Figure 2-32.

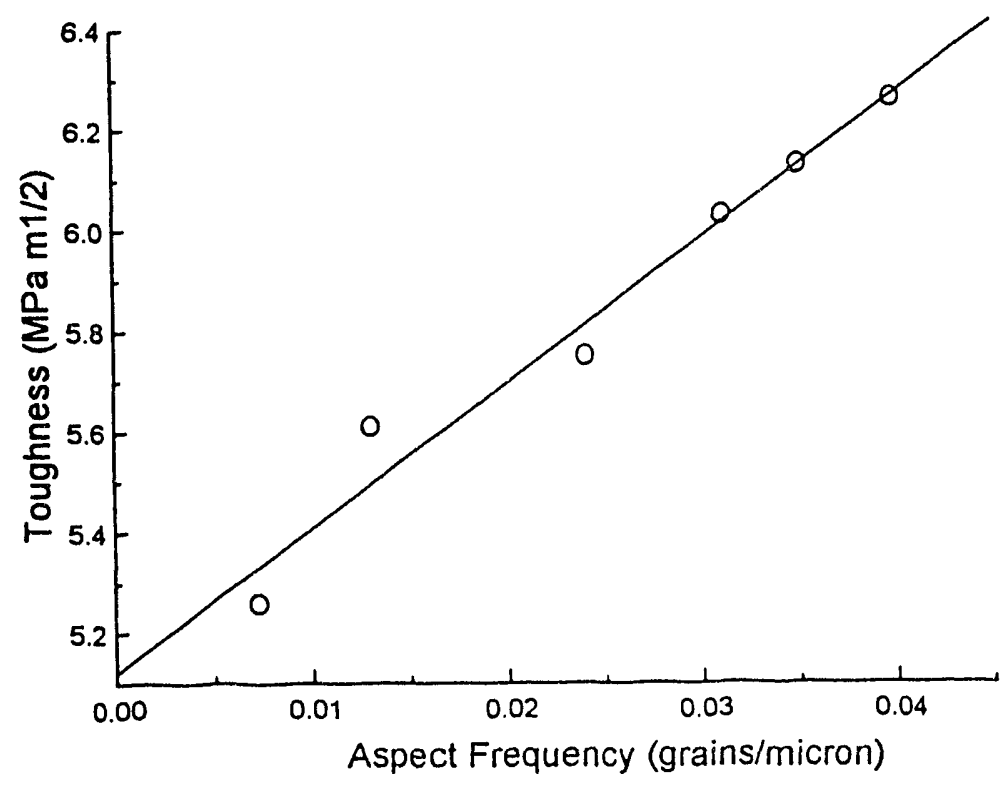

Figure 2-31: Fracture Toughness vs. Aspect Frequency 


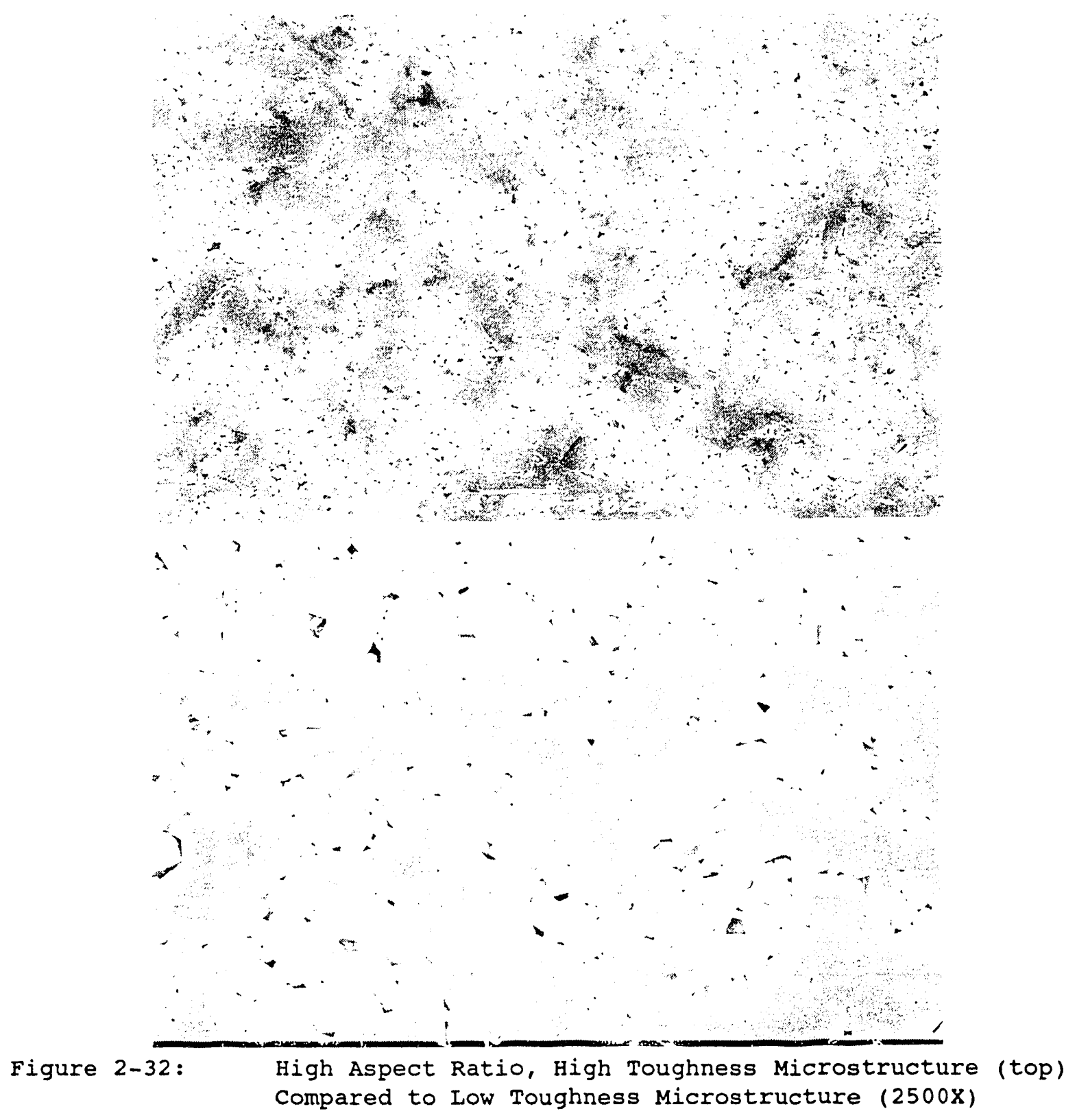

The HIP condition yielding high aspect ratio microstructure and corresponding fracture toughness $(6.26-6.6 \mathrm{MPa} \sqrt{ } \mathrm{m})$ was adopted as the standard process cycle for all stage III tensile bars.

REFERENCES

1. K.T. Faber and A.G. Evans, "Crack Deflection Processes - I. Theory," Acta.Metal. 31 (4) (1983). 


\section{APPENDIX 3: DEVELOPMENT AND APPIICATION OF NDE METHODS}

Several NDE methods were employed during the program to monitor the process, generate flaw population data, correlate these data to parameters of the unit operations and feed back the information for process improvement. Separate specialized reports have been written on the work performed in collaboration with subcontractors using ultrasonics ${ }^{[1]}$, magnetic resonance imaging(2), computed tomography ${ }^{[3]}$, and residual stress (4) analysis. In-house NDE R\&D efforts concentrated on microfocus radiography (MFX) and liquid dye penetrant (IDP) methods and highlights of this work are discussed below.

The initial task was to develop the techniques for application to various steps of the process. The detection capabilities of each NDE technique were determined quantitatively with appropriate reference standards which were prepared at NRDC.

\section{TECHNIQUE DEVELOPMENT}

Reference specimens were generated to determine the detection capabilities of the various NDE techniques. These specimens were used for the in-house NDE techniques as well as for the NDE pursued by the outside subcontractors. The reference specimens were prepared with defect types commonly encountered in samples from the various processing steps.

Bigh density inclusions in the form of Fe particles were seeded into Si3N4 specimens of constant thickness (tiles) as well as in tensile bars. The tiles were seeded with 75,100 , and $165 \mu \mathrm{m}$ Fe particles. Iron particles of $20,50,100$ and $200 \mu \mathrm{m}$ were seeded in the tensile specimens. The Si3N4 powder was mixed with various weight percentages of seeds precalculated to give a total defect number per sample. The mix was then CIP'ed into tiles and rods. Green, presintered and BIP'ed samples were prepared for the study. Green and dense Si3N4 samples of both geometries were used for detection sensitivity measurements on the microfocus $x-r a y$ unit.

Voids were simulated through the use of polymethyl methacrylate (PMMA) particles which were seeded into green tensile bars. The procedure for adding PMMA seeds was identical to that followed for the Fe inclusions. PMMA spheres of $20,50,100$ and $200 \mu \mathrm{m}$ were uniformly distributed into the samples. Voids were simulated by decomposing the spheres under suitable temperature and vacuum. Detection sensitivity of voids in green cylindrical samples was determined by microfocus radiography using these samples.

Probability of detection (POD) at 958 confidence level was determined using the above seeded reference specimens. In the HIP'ed state $\mathrm{Fe}$ inclusions as small as $20 \mu \mathrm{m}$ were detected at lower bound probability of detection (P1) of $<0.2$ (thickness sensitivity $<0.38$ ); while at thickness sensitivity of $>2.38, \mathrm{Pl}=0.97$. In both the green and presintered state $P 1>908$ values were obtained for thickness sensitivity $>2 \%$. Minimum Fe particles detected were around 20 um (P1) around 0.20$)$. Point estimates of the probability of detection were higher for HIP'ed Si3N4. In green samples, a 28 thickness sensitivity was achieved for lower bound probability of detection $>908$. 
Microfocus detection data on simulated voids yielded P1 > 958 for thickness sensitivity of 58 and higher for both presintered/ green Si3N4 samples. In the green/presintered state, voids as small as $100 \mu \mathrm{m}$ were detected at low P1 $(<0.2)$.

specimens containing known density gradients were prepared in green and dense cylindrical samples. Three Si3N4 powders having different particle sizes were CIP'ed together to produce green rods with density gradients of 2,6 and 88 . The gray level differences between the different density zones were easily identified by microfocus radiography. Density gradients were formed in dense Si3N4 samples by varying the amounts of sintering aid in three powders and then CIP'ing a compact from the powders. The dense compact had three distinct density zones with densities differing by 1,2 and 38 . The samples were machined symmetrically and $x$-rayed. The three density zones were clearly detected by microfocus radiography, validating this approach of density gradient evaluation.

Agglomerates were seeded in both loose packed Si3N4 powder as well as in Si3N4 slips for detection studies. The agglomerates were classified from spray dried $\mathrm{Si}_{3} \mathrm{~N}_{4}$ powder into different size fractions using steel sieves. Agglomerates were sized to $400,140,100,60$ and 40 $\mu \mathrm{m}$. The agglomerates were seeded into either a $8 \mathrm{~cm}^{3}$ container of $\mathrm{Si}_{3 \mathrm{~N}}$ powder having a particle size of $<20 \mu \mathrm{m}$ or a $8 \mathrm{~cm}^{3}$ container of a submicron colloidal slip. The container was half filled with either dry powder or slip, seeded with the agglomerates, then the remainder of the vial was filled with slip or powder.

The agglomerates were successfully detected in both the slip and powder. The detection limits of agglomerates in loose packed Si3N4 powder was $60 \mu \mathrm{m}$ (2.48 thickness sensitivity) compared to $100 \mu \mathrm{m}$ (48 thickness sensitivity) in Si3N4 slurry. The same size container was used during the program to analyze powders and slips for agglomerates.

A standard procedure was developed to detect cracks using fluorescent dye penetrant on cylindrical Si3N4 reference samples. cracks were introduced using the vickers indentation technique. Indent loads of 2.5 - $40 \mathrm{~kg}$ produced cracks varying in length from $14-220 \mu \mathrm{m}$ which were measured using optical/SEM microscopy. The samples were liquid dye penetrant tested with a post-emulsifiable penetrant with level IV sensitivity. The technique was optimized until crack lengths as small as $14 \mu \mathrm{m}$ could easily be detected. The crack length measured with the SEM was compared to that measured from IDP and results are plotted in Figure 3-1. The cracks measured with IDP were approximately $20 \mu \mathrm{m}$ larger due to bleed out of the penetrant.

Film digitization and image processing capability was developed to quantify flaw populations in tensile specimens. The most useful feature was found to be contrast enhancement using gray level slide/stretch algorithms (adaptive thresholding). Gray level thresholding of a digitally enhanced image at selected areas on the radiograph has allowed counting and sizing of features such as metallic inclusions. This technique worked very well on radiographs whose background had an intrinsic variation due to variation of thickness. Background subtraction techniques were very useful in the identification of voids near the edge of the sample. Due to gray level differences in digital images of radiographs, only small areas of interest could be analyzed at 
one time. These gray level non-uniformities are caused by nonuniformity of the beam intensity and the unevenness of the illumination when capturing the inage. For these reasons, it was very time consuming to analyze the entire radiograph and to perform automated counting of the flaws.

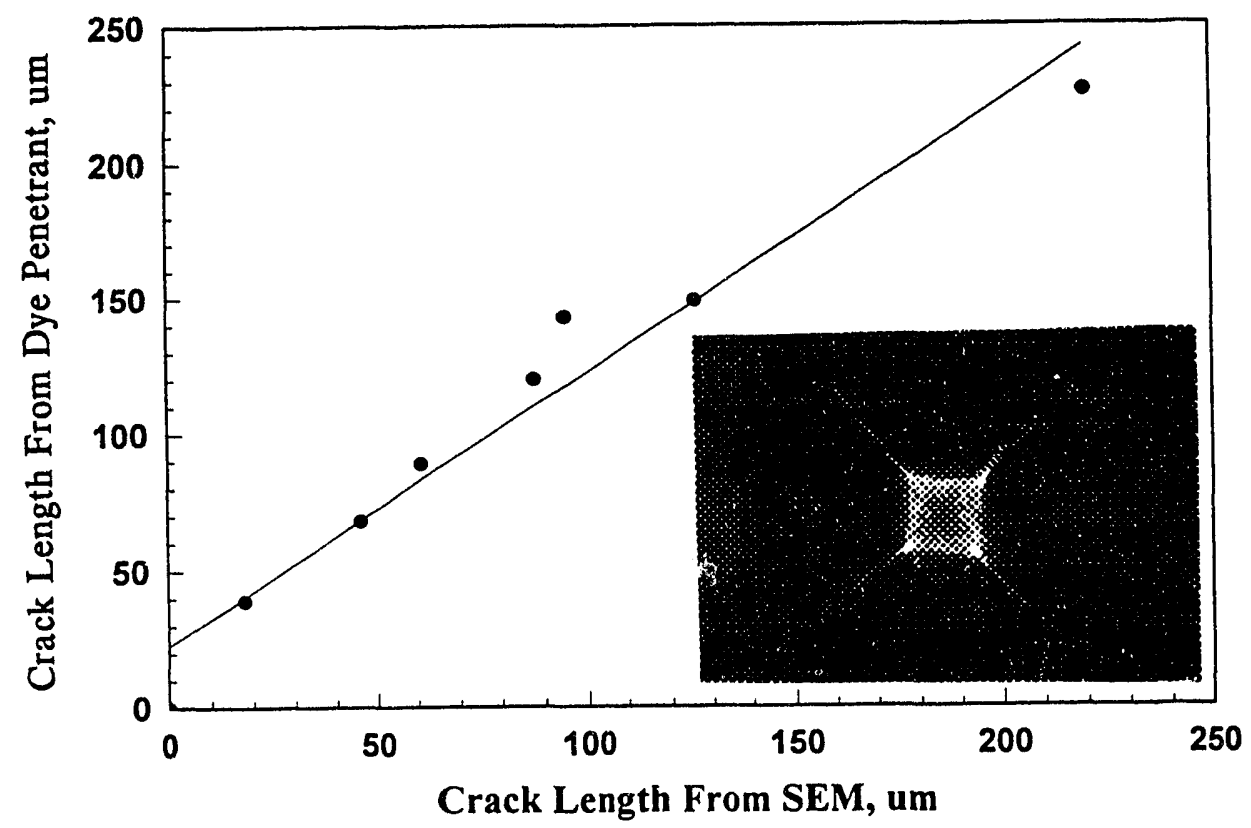

Figure 3-1: Comparison of vickers Indent Crack Length Measurements, IDP VS. SEM

Experimental design was adopted to optimize the film exposure conditions so maximum contrast could be achieved to improve the detection sensitivity. It was found that the use of fine-grain films at particularly low values of excitation voltage and current gave the optimum contrast/resolution. At low energy levels, the absorption coefficient difference between $\mathrm{Si}_{3} \mathrm{~N}$ and Fe differs by a factor of 20 . several types of anodes (Mo, $\mathrm{Fe}, \mathrm{Cu}$ ) were evaluated but no beneficial advantages were seen over tungsten in detection capabilities. Mating surfaces of Si3N4 were made to conform to the cylindrisal shape of the tensile rod. This was intended to offset the thickness gradient found in cylindrical samples, but provided no practical advantages. The combination of low tube current and high filament current proved to provide the best resolution due to less geometric unsharpness. Even though low energies were utilized, the use of thin filters vastly improved definition and increased edge detection on cylindrical samples by as much as 278 .

\section{INJECTION MOLDING PROCESS NDE SUPPORT}

Microfocus radiography was instrumental in developing the injection molding technique. As-received Si3N4 powder, binder, compounded mix, pelletized $\mathrm{mix}$, green NS $\mathrm{I}$ bars, debinderized/presintered NSF bars and HIP'ed NSF ba:s were all analyzed by microfocus radiography 
for the identification of flaw populations.

The binder used in the injection molding mixes was evaluated for high density inclusion contaminants, since such binders are manufactured for the plastics industry where these types of flaws are of no concern. The binder " $D$ " used in the baseline iteration was processed through a set of filters and then radiographed at $10 x$ magnification for detection of inclusions. A series of 9 experiments were carried out using a variety of filter set-ups. The final filter system tested yielded a factor of 10 reduction in the number of inclusions.

Compounded and pelletized mixes were also examined by microfocus x-ray for the presence of high density inclusions. These types of inclusions are in part products of wear from the processing equipment. The compounded mix was prepared by rolline, it into a flat disc (pancake) before examining it. The pelletized mix was examined in the form of 5 mm diameter pellets. The compounded and pelletized mixes were both found to contain $\mathrm{Fe}$ inclusions in approximately 308 of the samples analyzed. The data on size distribution and frequency of inclusions for the compounded and pelletized mixes are shown in Figure 3-2. Inclusions from $35.400 \mu \mathrm{m}$ were detected in the compounded and pelletized samples.

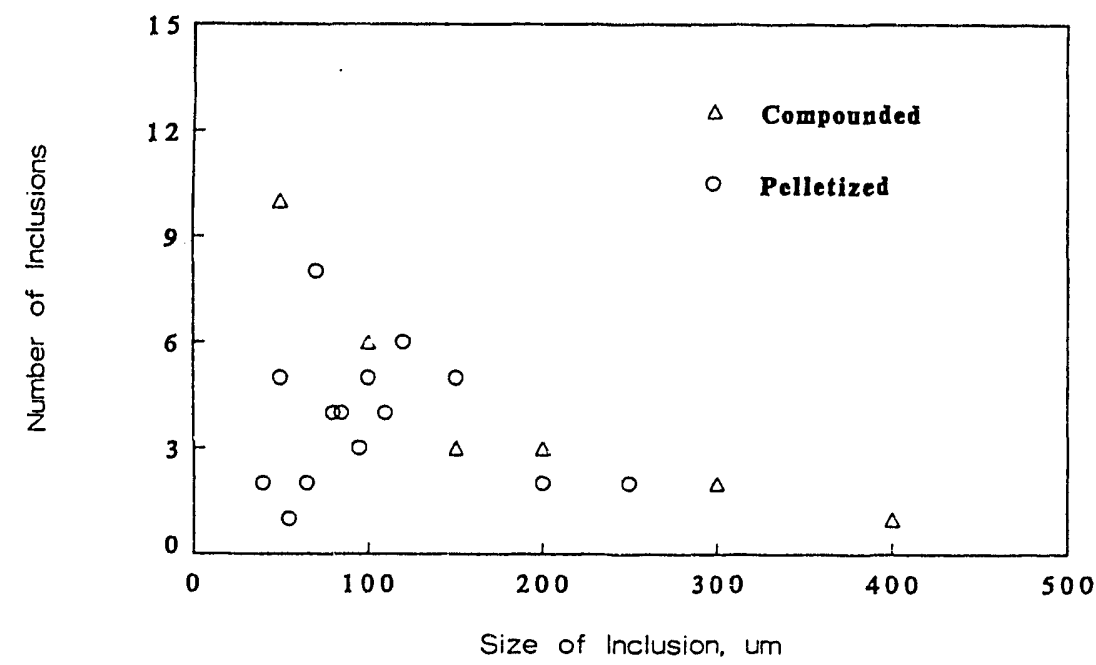

Figure 3-2: Metallic Inclusions Detected by MFX Kadiography in Injection Molding Process

Microfocus film radiography of injection molded, debinderized and presintered tensile rods was performed. The radiographs were taken of the entire tensile rod at $2 \mathrm{x}$. Each set of rods was shot at two angles $(0,90$ degrees) to allow determination of whether defects were surface/near surface or bulk and to also ensure that those defects unfavorably oriented to the $x$-ray beam at one angle were detected.

At the early stages of development in the injection molding sequence, microfocus $x$-ray was used to set the molding parameters. Process parameters such as temperature, pressure, gating of molu, etc. were analyzed by examining the flaw population. For example, three different size feed orifices to the mold (D7, D9, D11) were evaluated by examining the void population in the molded rods. The orifice size chosen was based on the radiography data. Microfocus film radiography 
evaluation of the as-molded tensile rods of the first iteration found an average flaw indication per sample of around two, which represented a significant improvement over earlier molding experiments which had average flaw per sample values closer to six.

There were approximately 300 injection molded rods $x$-rayed in the green state. The majority of the flaws were voids located in the buttonhead section of the rod. The other prominent flaw population was high density inclusions ( $\mathrm{Fe}$ ) found predominantly in the gage section. Nearly 57t of the rods tested had detectable voids and 458 of rods had inclusions. The average void size was $>500 \mu \mathrm{m}$ and the size range of the inclusions was 200-500 $\mu \mathrm{m}$.

In the HIP'ed state, all tensile rods were 1008 examined by microfocus $x$-ray and liquid dye penetrant. Film radiography was performed in the gage area at a magnification of $2 x$. All the liquid dye penetrant examinations were at 1-40X magnification on the entire test specimen. The liquid dye penetrant testing was done using a level III fluorescent post emulsifiable penetrant. The emulsification step was carried out with the hydrophilic process. A total of 94 HIP'ed rods were evaluated with NDE. Inclusions ( $\mathrm{Fe}$ ) were determined to be the major flaw population. Over 708 of the rods examined were found to contain inclusions with an average size of $200 \mu \mathrm{m}$. On'? 18 of the samples tested had voids suggesting HIP'ing healed the porosity observed in the green state. Figure 3-3 illustrates the size distribution of the inclusion flaw population for iteration 1. The major flaw population detected by LDP were cracks in the buttonhead section of the tensile specimens. A few of the specimens that had cracks detected by LDP were $x$-rayed to confirm whether or not it would be detected by this technique. This test was performed on 35 samples, and in every case, IDP results correlated with microfocus $x$-ray. The cracks seen in the buttonhead were not considered detrimental if they were not in the vicinity of the buttonhead radius where the bar is gripped for testing. Figure 3-4 is an example of a crack detected by LDP in the buttonhead region of the rod. A total of 47 rods had cracks that were potential failure sites. The other flaw population observed with LDP was machining damage. Figure 3-5 is an example of the type of machining damage observed in the transition section of a tensile bar.

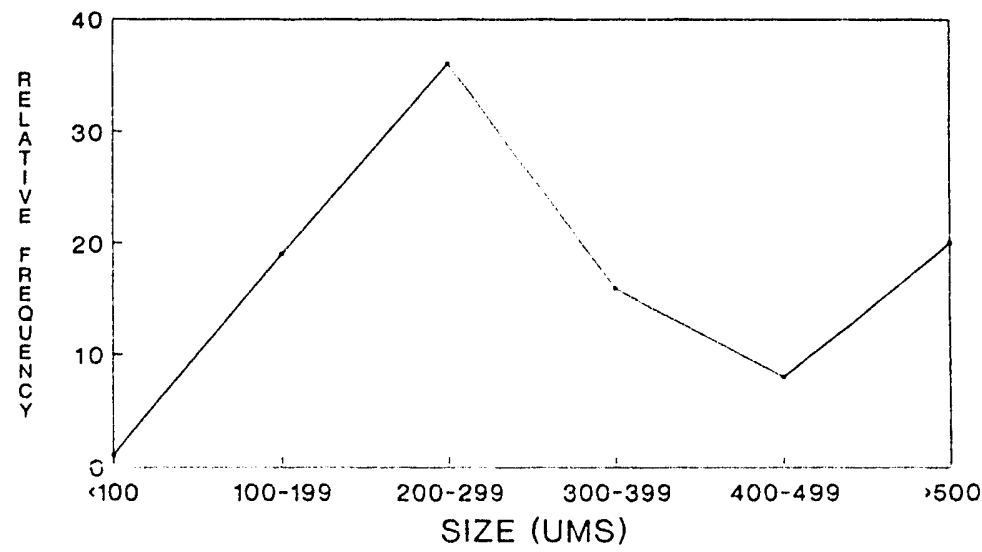

Figure 3-3: Relative Frequency of Metallic Inclusions Detected in HIP'ed Injection Molded NSF Bars 


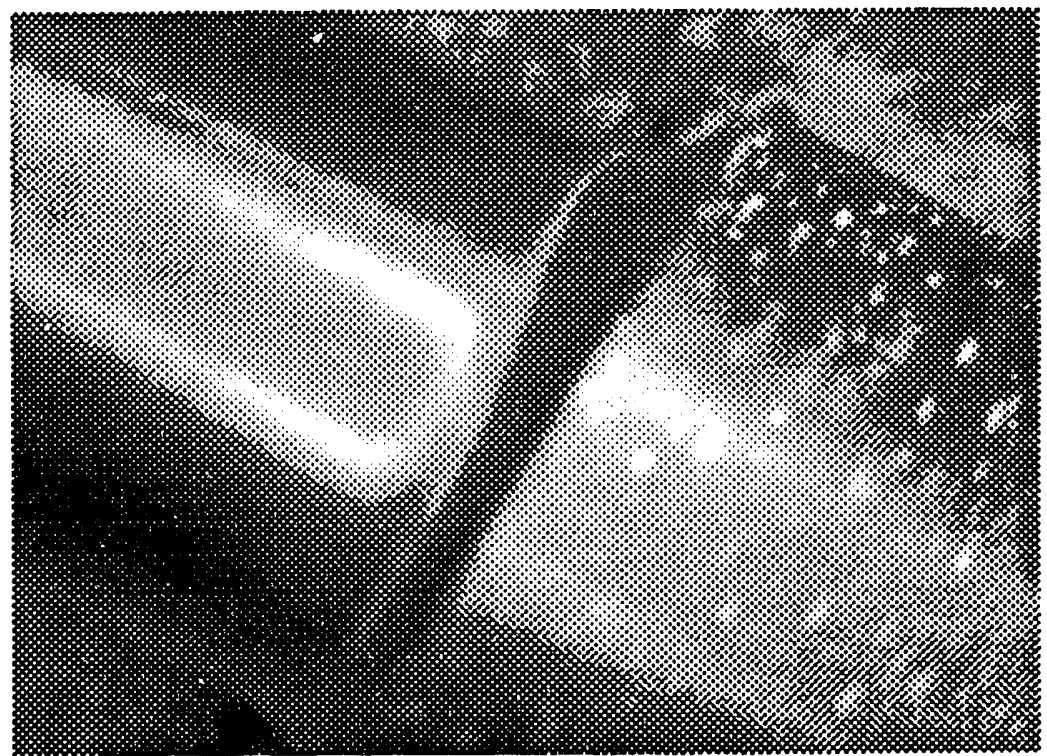

Figure 3-4: Buttonhead Radius Crack (14 mm) Detected by LDP

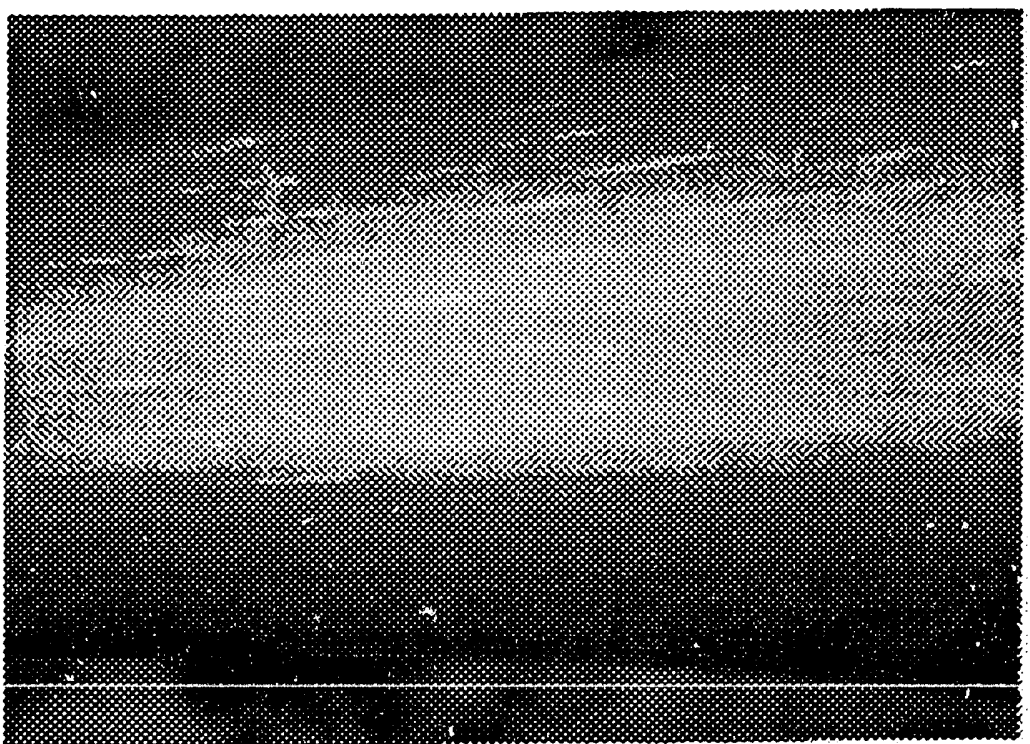

Figure 3-5: Machining Danage in Transition Region of NSF Bar Detected by LDP 
Several mini-iterations (20-25 rods) were carried out after the first baseline iteration in order to refine the injection molding process. This was pursued in an effort to reduce the size and frequency of the flaws as well as improve on the overall mechanical properties. Early experience had voids concentrated at one end of the mold in the buttonhead section of the rod. Injection molded specimens from miniiteration Batches I and I were radiographed as-molded. Batch I was produced using binder which had been screened for inclusion control. Furthermore, on the basis of void and crack frequency observed in Batch I specimens, molding parameters were adjusted for Batch I. Radiography confirmed that the adjustments were successful since these defects were greatly reduced relative to Batch I, Figure 3-6.

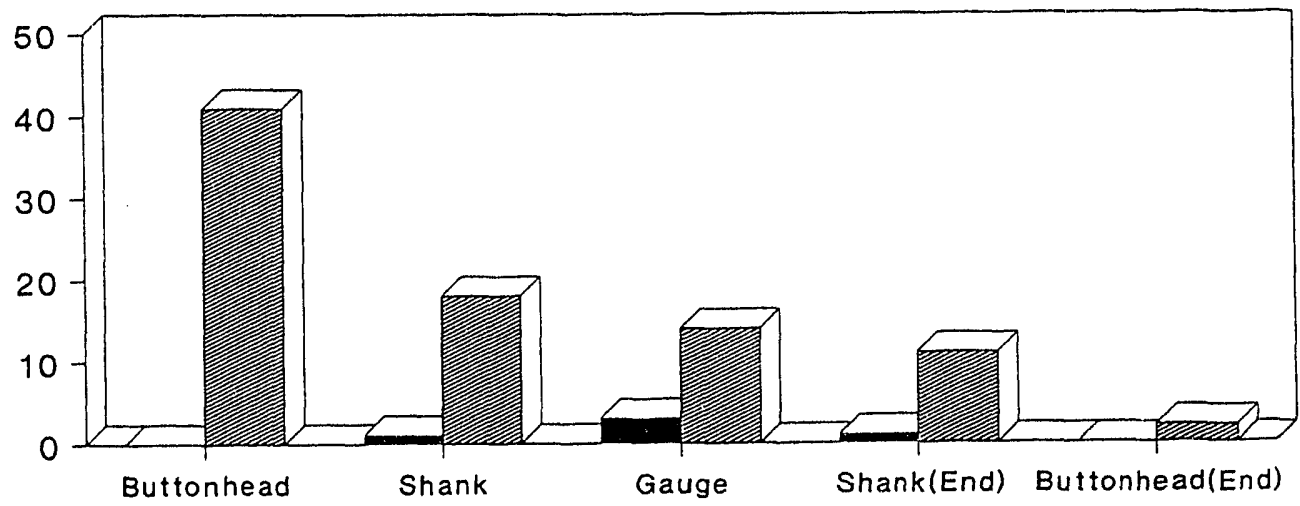

\# of Voids-Batch L W of Voids-Batch 1

Figure 3-6: Void Frequency and Distribution in As-Molded NSF Bars.

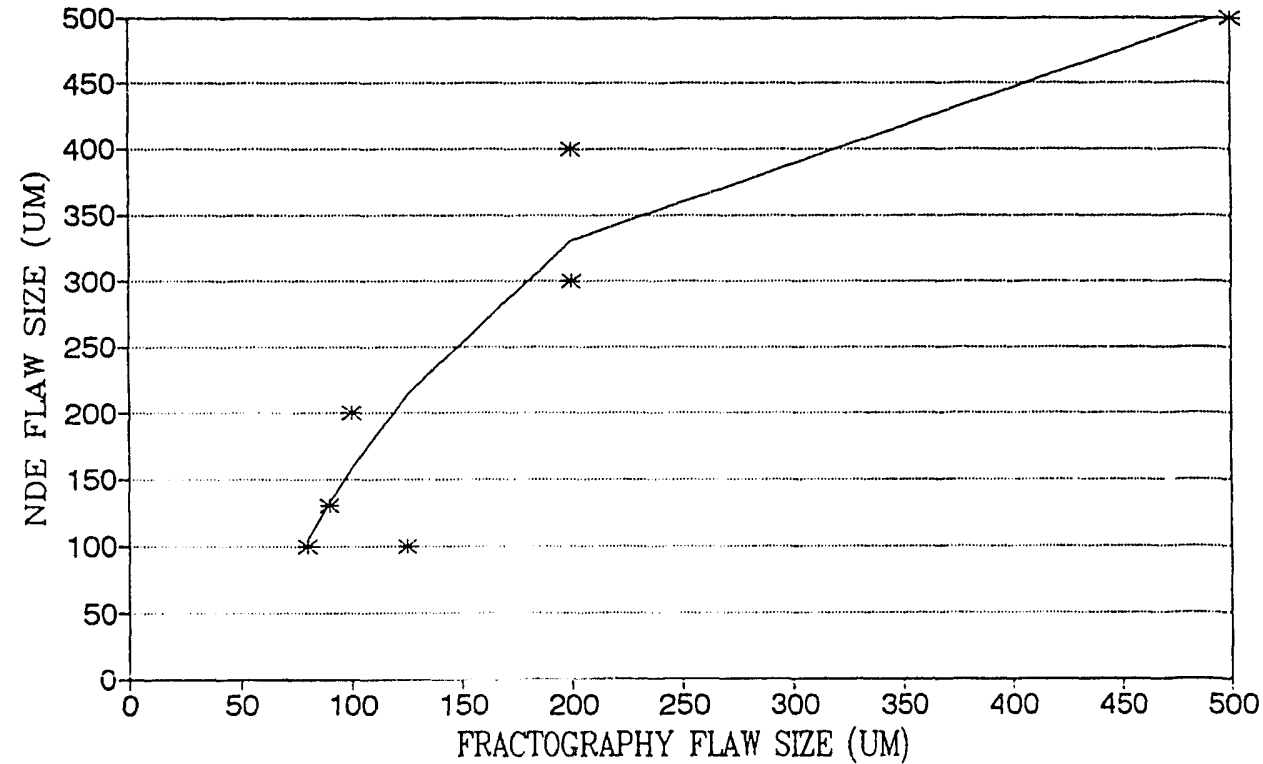

Figure 3-7: NDE/Fractography Inclusion size Comparison 
Inclusion population data gathered by microfocus $x$-ray were compared to fracture origin data collected with the aid of SEM. Flaws found by NDE before testing were compared to the critical flaws identified post-mortem by fractography/sEM. Flaw size at the failure origin of those rods which was identified by both techniques is plotted in Figure 3-7. The plot suggests reasonable correlation between the flaws measured by NDE and the fractography data.

COLLOIDAL CONSOLIDATION - BASELINE ITERATION - NDE SUPPORT

Pressure cast samples were analyzed by microfocus $x$-ray at all stages of processing. In the early stages of casting development, a preponderance of voids in green NSF bars was identified as a major quality problem. Radiography was used to monitor the effectiveness of casting procedure alternations by tracking the decrease in void population at each state of development.

The predominant defects in the green samples were voids which had an average size of $375 \mu \mathrm{m}$. Voids of this size or smaller were no longer evident after HIP'ing. Inclusions in the $100 \mu \mathrm{m}$ size range were the major flaw type detected in HIP'ed samples. Film microfocus radiography was also used extensively for evaluation of density variations within NSF bars. Cross-sections of green NSF rods were radiographed to establish how density gradients and also casting fronts develop as a function of casting parameters such as filtration surface, mold material, etc.

The size distribution of inclusions for both the injection molding and pressure casting baseline iterations is plotted in Figure 3-8. As illustrated, the average inclusion size found in pressure cast samples was $100 \mu \mathrm{m}$ compared to $200 \mu \mathrm{m}$ for injection molded samples.

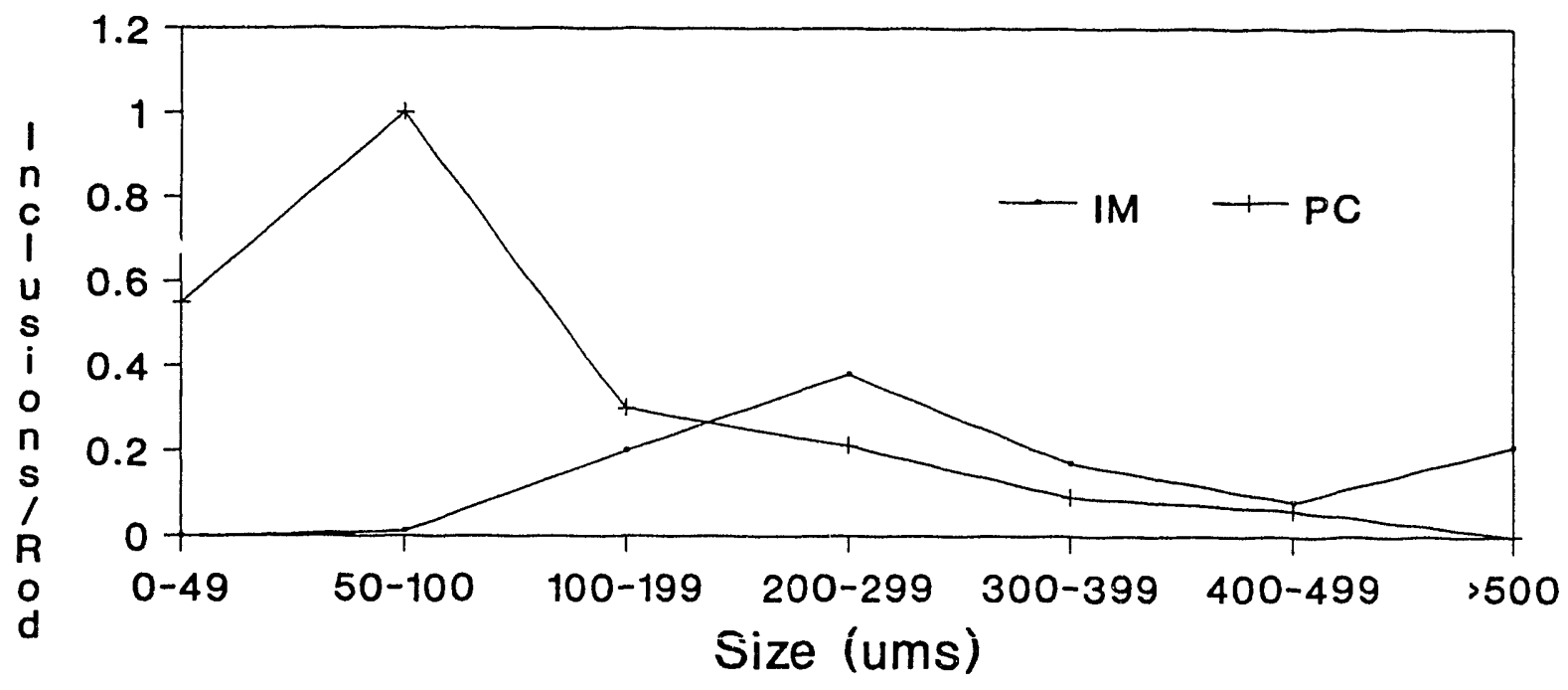

Figure 3-8: Inclusion Size Frequency in HIP'ed NSF Bars From Injection Molding and Pressure Casting Baseline Iterations 
STAGE II PROCESS OPTIMIZATION NDE SUPPORT

specimens from all stages of processing were examined by microfocus radiography during stage II in order to monitor the process. This includes all processing steps from the starting powder to the final machined NSF bar. Iiquid Dye Penetrant was used as a surface analysis tool for the identification and measurement of surface damage on HIP'ed/machined specimens. Stage II was also used to further optimize the NDE techniques for different specimens at each stage of processing. The techniques were optimized in order to achieve the best contrast and resolution between the flaw type and the bulk material.

As-Received Powder

As-received UBE powders were analyzed by microfocus radiography for the presence of metallic inclusions. At the beginning of stage II the major flaw population governing failure were metallic inclusions. The powders were examined by microfocus $x-r a y$ since they were thought to be a contributing source to the contanination. Even though the powders meet the purity specifications, they did contain a few large particles of the impurity. Samples $(40 \mathrm{~g})$ were taken from each barrel that contained a particular particle size (EO3, EO5, E10) for each lot of powder. This was pursued to determine whether a particular particle size lent itself to more contamination. No conclusive results were found to confirm this hypothesis. A total of 15 samples were examined from one $50 \mathrm{~kg}$ barrel of EO3, Lot D UBE powder. There were 10 inclusions found ranging in size from $25-200 \mu \mathrm{m}$. Samples from six 50 $\mathrm{kg}$ barrels of as-received Lot $C$ UBE powder were radiographed and two samples were found to contain single $50 \mu \mathrm{m}$ inclusions. The magnetic separation stage of the milling precess was considered adequate to remove this source of contamination from the process.

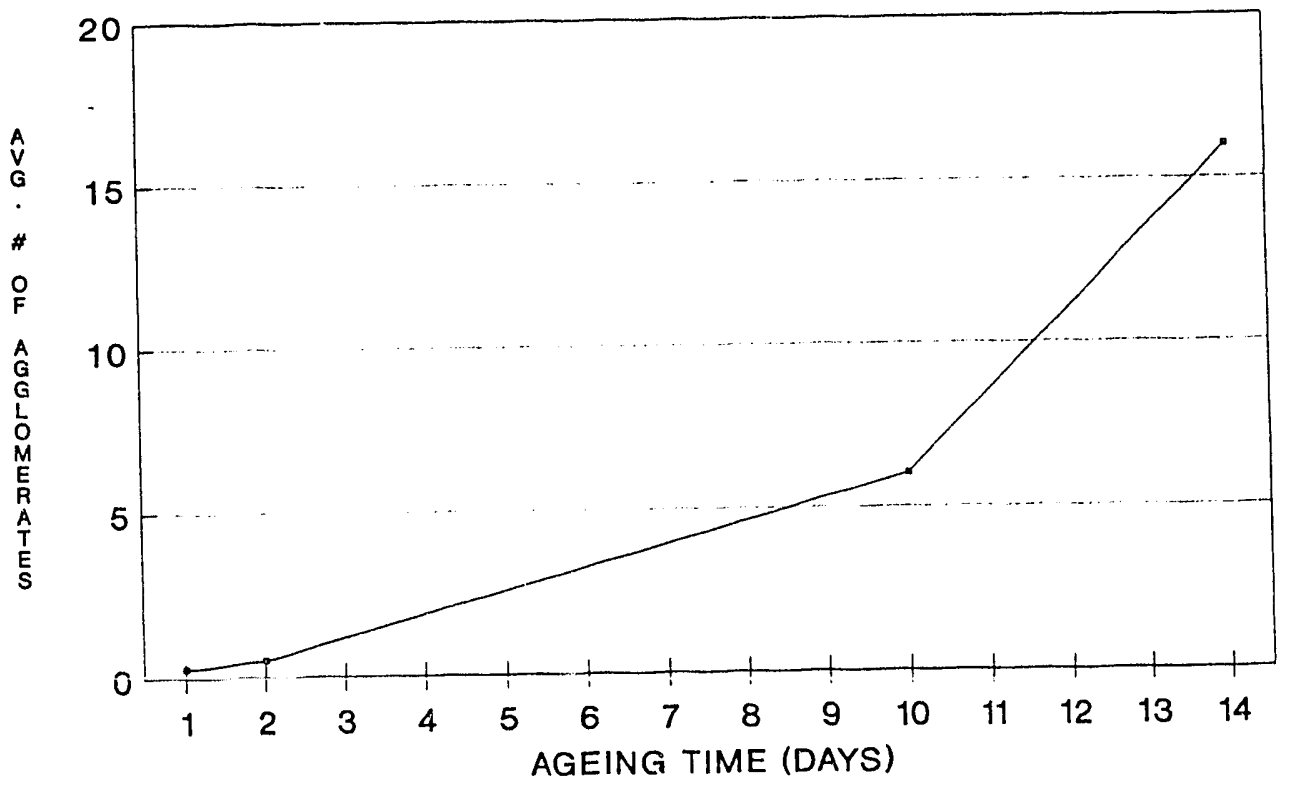

Figure 3-9: Aging/Agglomeration of slip Detected by MFx Radiography 


\section{Colloidal suspensions}

Colloidal suspensions from mill batches $\operatorname{coll}$ - $\operatorname{co2} 9$ were examined for the presence of agglomerates and inclusions. The slip was transferred into $33 \mathrm{~cm}^{3}$ plastic containers and examined at $10 \mathrm{x}$ using microfocus radiography. The evaluations were performed during the period that the casting operation was being conducted (within hours of milling). Agglomeration was found to occur with aging of the slip. Samples were radiographed 1.5 - 2 weeks after slip preparation and agglomerates $100-500 \mu \mathrm{m}$ in size were detected, Figure 3-9. These findings prompted a change in the casting SOP to require fresh slip for the casting process. No agglomeration was observed in any of the slips during stage II when examined within 72 hours of milling. Ordinarily, the casting operation was scheduled to consume the slip in 48 hours.

\section{Presintered NSF Bars}

Presintered NSF bars from iterations N, O, P, Q, S, T, U, V, X, Y, $Z$, and $A A$ were examined during stage II. MFX was used to establish casting controls as they relate to air entrapment and the resultant void defects. The examination of green specimens was performed at $4 \mathrm{X}$ magnification at the beginning of the program. As the average flaw size started to decrease, the magnification was increased to $9 \mathrm{X}$, as was the case with iterations $z$ and $A A$. A variety of flaw types were found in the gage section of the bars, including low density pores, agglomerates, cracks, and high density inclusions. The majority of the flaws seen were low density pores or voids. Flaw population charts based upon specific flaw counts were generated for each batch of green specimens.

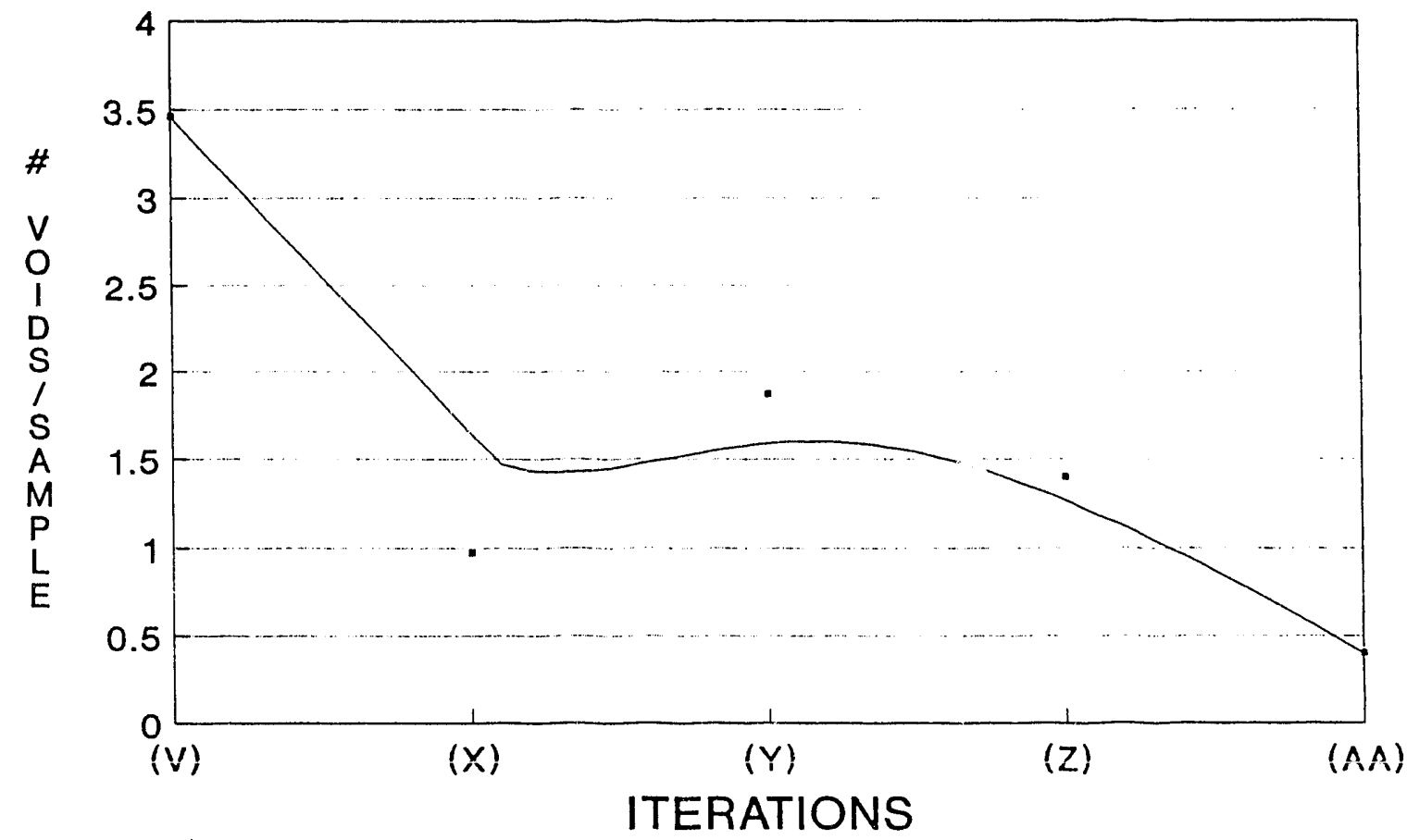

Figure 3-10: Void Frequency in As-Cast Tensile Rods for Casting Iterations $\mathrm{V}-\mathrm{AA}$ 
An automated closed loop casting unit was installed at the commencement of Iteration $x$. significant quality improvement was realized as can be seen in the plot of \# voids/sample for iterations $V$ through AA in Figure 3-10. There was a reduction in void frequency from a level of 3.5 to 1.5 voids/sample. The $1.5 \mathrm{void} / \mathrm{sample}$ level of iterations $X, Y, Z$ was reduced to a 0.5 level for iteration AA, after minor adjustments of the machinery.

\section{HIP'ed NSF Bars}

The non-destructive examination of dense samples was conducted after final machining. The HIP'ed/machined specimens were analyzed by both MFX and IDP in the gage section. At the initial stages of stage II, all microfocus $x$-ray work was performed at $5 x$. Later, as the size of the flaws decreased, the magnification was increased to $10 x$ for all future work.

The improvement in processing over the course of the program can be plotted in terms of flaw population data versus iteration. Figure 311 provides plots of average flaw size and number of flaws per sample for the first 9 iterations. The flaws include pores, inclusions and agglomerates detected in the gage length volume. The plots indicate dramatic improvement in both size and frequency which corresponded to strength improvements. Another illustration of process improvement is provided by data documenting the percentage of bars which had defects detectable by MFX. Whereas in excess of 808 of baseline iteration specimens had detectable flaws, by the ninth iteration this figure was less than 208

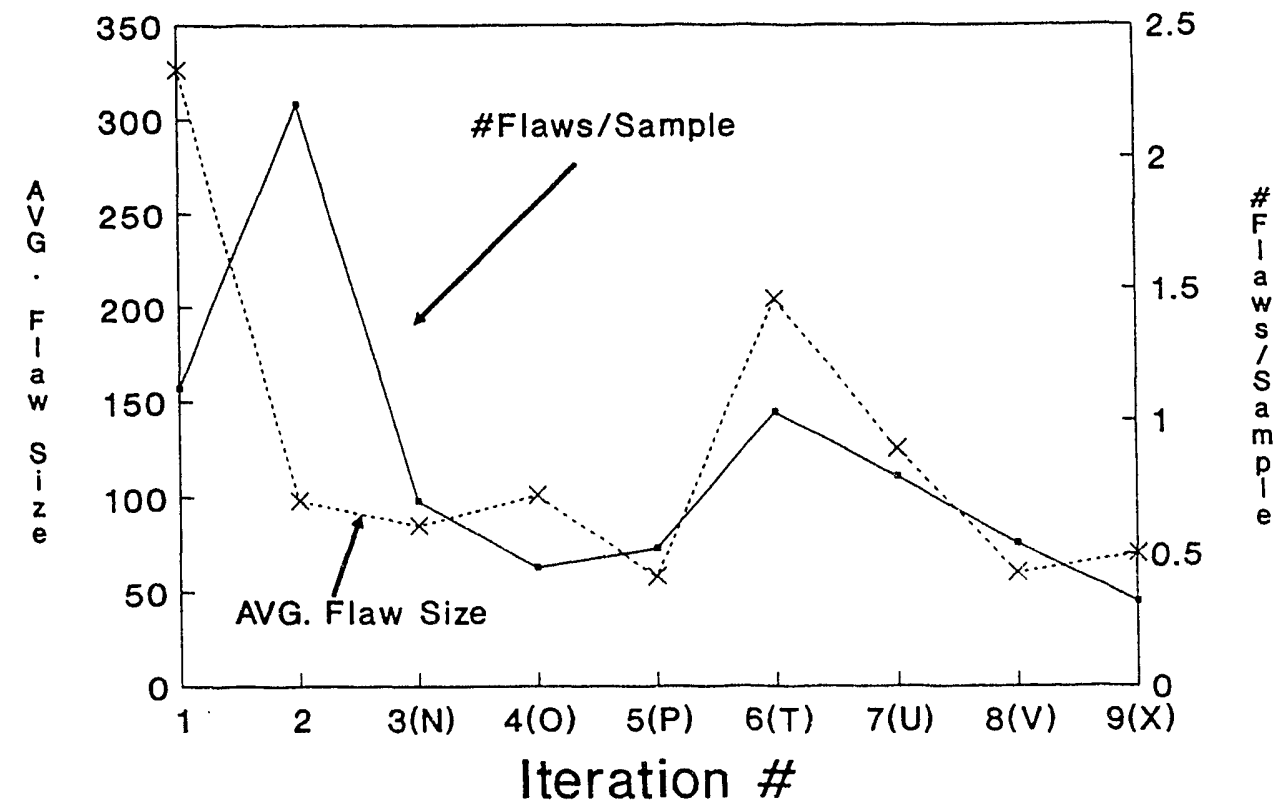

Figure 3-11:

Flaw statistics from MFX Evaluation of Iteration Samples 
Approximately 508 of the NSF bars from iterations 3, 4, and 5 showed no flaw indications and as Figure 3-11 suggests, there was roughly one flaw averaging $75 \mu \mathrm{m}$ in each of the samples which had indications.

The inclusion flaw populations for iterations $T-z$ are plotted in Figure 3-12 in the form of \# inclusions/sample and 8 rods with inclusions. After Batch $T$ was processed, iron contamination was discovered in the milling and casting equipment. The iron contamination was associated with the wear and corrosion of the metallic components. At that point, replacement components were redesigned with organic materials to circumvent the problem. As can be seen in the graph, the metallic contamination was decreased drastically. Whereas more than 508 of Iteration $T$ rods were found to contain metallic inclusions, only 78 of Iteration $z$ samples were found to have these defects. The equipment wear influence on Batch $T$ is also evident in Figure 3-11, where there was a sudden rise in the average flaw size and the percentage of rods with flaws. As more and more wear areas of the process equipment were replaced, there was a gradual decrease in the flaw population from batch $T$ to batch $X$. This is a good example of how the results of NDE led to the discovery of a problem in the process equipment.

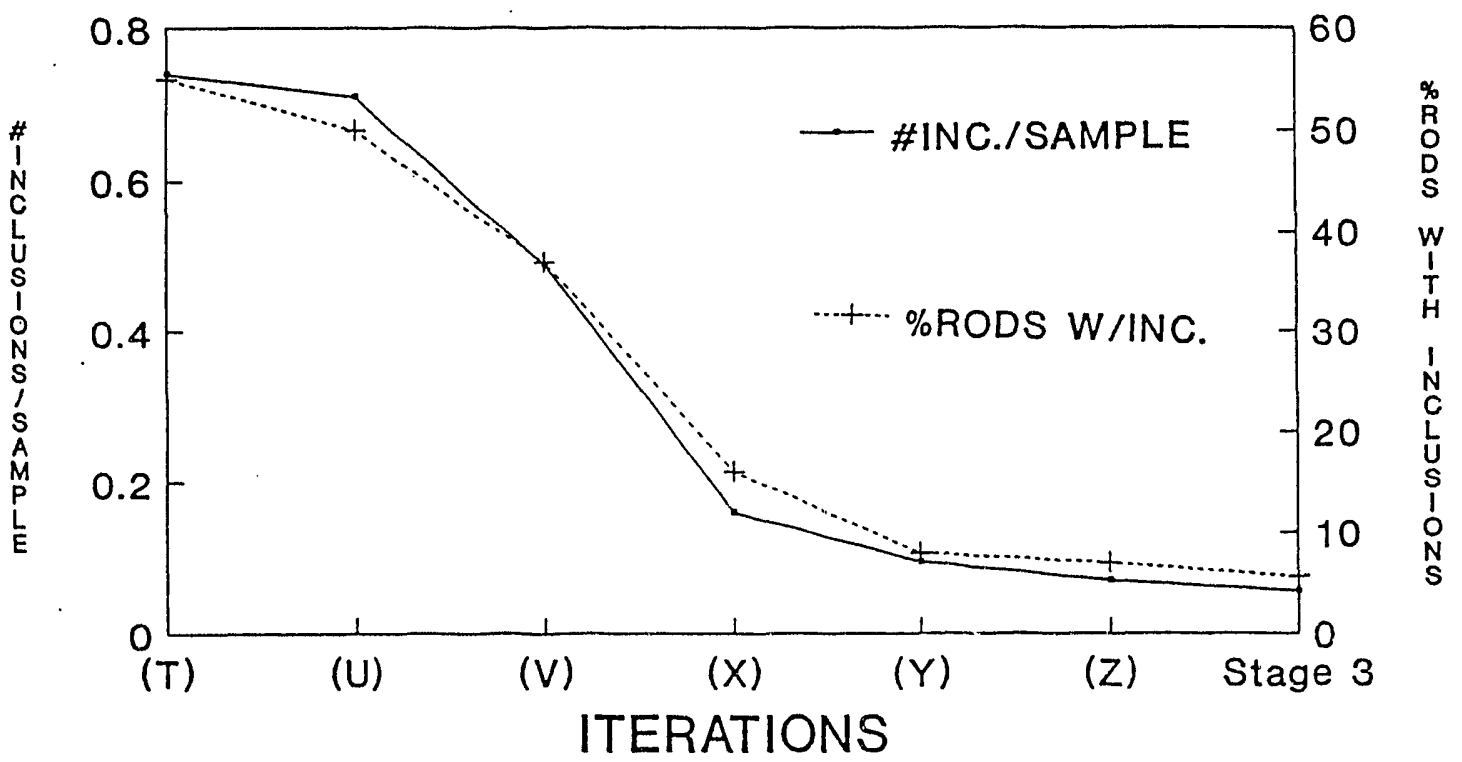

Figure 3-12: Inclusion Population Statistics During Stage II and III Iterations

\section{Density Gradient Evaluation}

During the course of Stage II, specimens from several different batches were MFX X-rayed to identify density gradients. Thin crosssections were sliced from presintered green rode with flat and parallel faces. Specimens were $x$-rayed to measure the effects of different casting parameters, mold materials, pressure profiles, and filtration surfaces on the formation of density gradients. For example, an x-ray of a specimen cast in a two piece mold, where only $1 / 2$ the mold was $a$ 
filtration surface, revealed a low density region along the drawing part of the mold. This technique was very useful in refining the casting process.

A sintered Si3N4 step wedge was made with steps that varied in thickness by 18 to simulate density gradients. The thickness of the wedge was approximately that of the gage section of a HIP'ed tensile rod. The wedge has 7 steps and its thickness varied from .2345 to .2500 inches. The step wedge was examined using film radiography and all steps were clearly seen as shown in Figure 3-13.

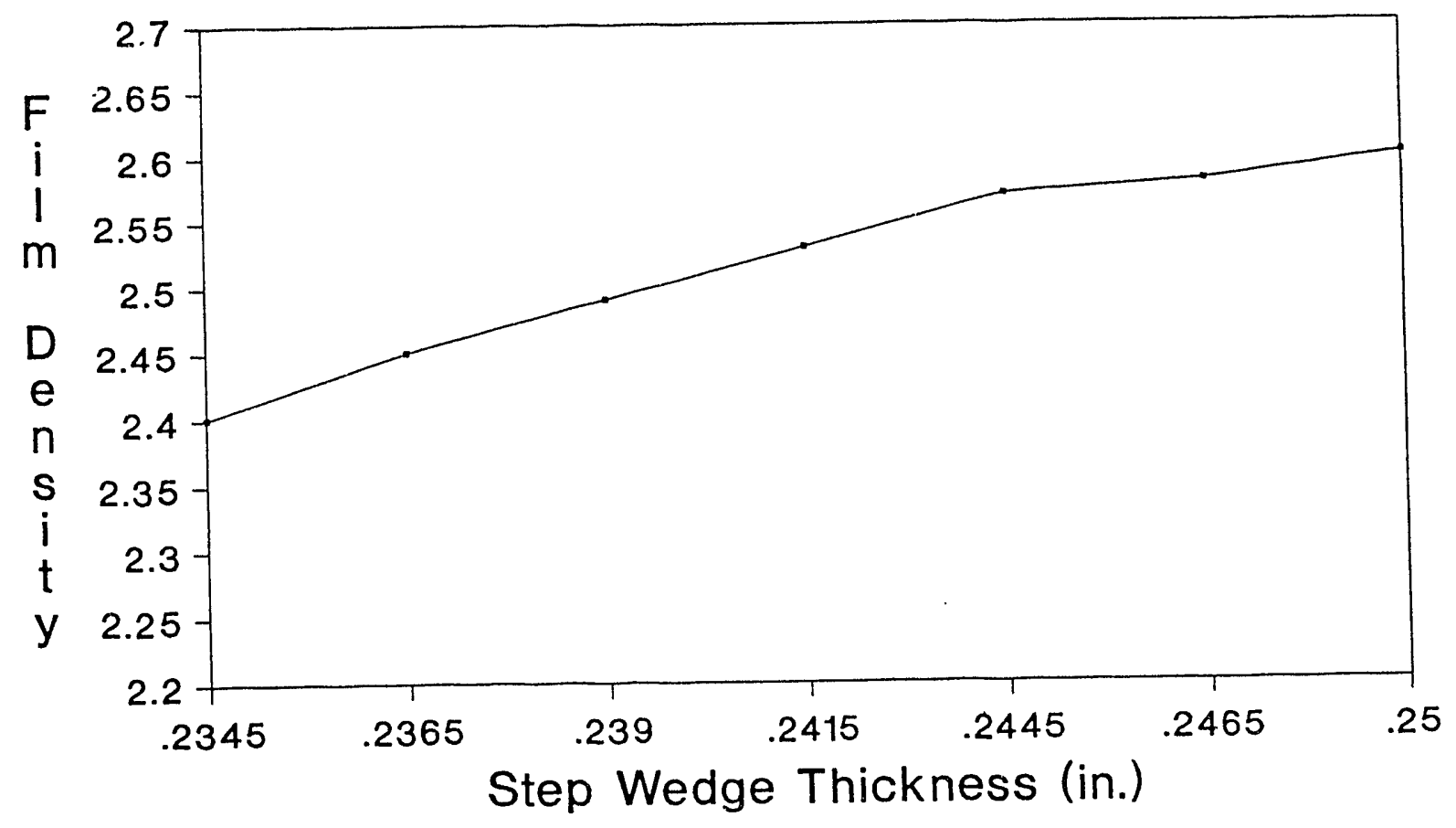

Figure 3-13: MFX Radiography Density Gradient Calibration Si3N4 Step Wedge

\section{Strength-Flaw Correlation}

The machined NSF bars were also examined for strength impairing flaws for correlation with fractographic analysis. A number of NSF bars from Iterations $T, V$ and $X$ failed from inclusions during tensile testing, corresponding to the metallic contamination that has previously been discussed. Radiographs of those specimens which failed in the gage length were used to establish a possible relationship between inclusion size and NSF bar strength. Fracture mechanics suggests the relationship

$$
\sigma_{f}=c \frac{K_{1 c}}{\sqrt{a}}
$$

where the constant c represerts the geometrical features of the defect 
with dominant dimension a. In this case, a was taken as one-half the largest inclusion dimension perpendicular to the tensile axis which was apparent in the radiograph. A penny shaped crack would have a value of 0.89 for the constant $c$.

Given the flaw size from the radiograph, a predicted value of of was computed for each specimen using equation $(3-1)$ and this was compared to the actual test strength. A value of $6.2 \mathrm{MPa} / \mathrm{m}$ was used for Ric in this computation. The value considered to be the best choice for the constant $c$ was that value which gave a regression line which best approximated the predicted = actual line, Figure 3-14. As can be seen, although there is considerable scatter in the data, the regression line with $c=0.65$ corresponds almost exactly to the $45^{\circ}$ line in this plot. The value of 0.65 suggests that the effect of an inclusion in Si3N4 is more severe than a penny shaped crack of equivalent dimension.

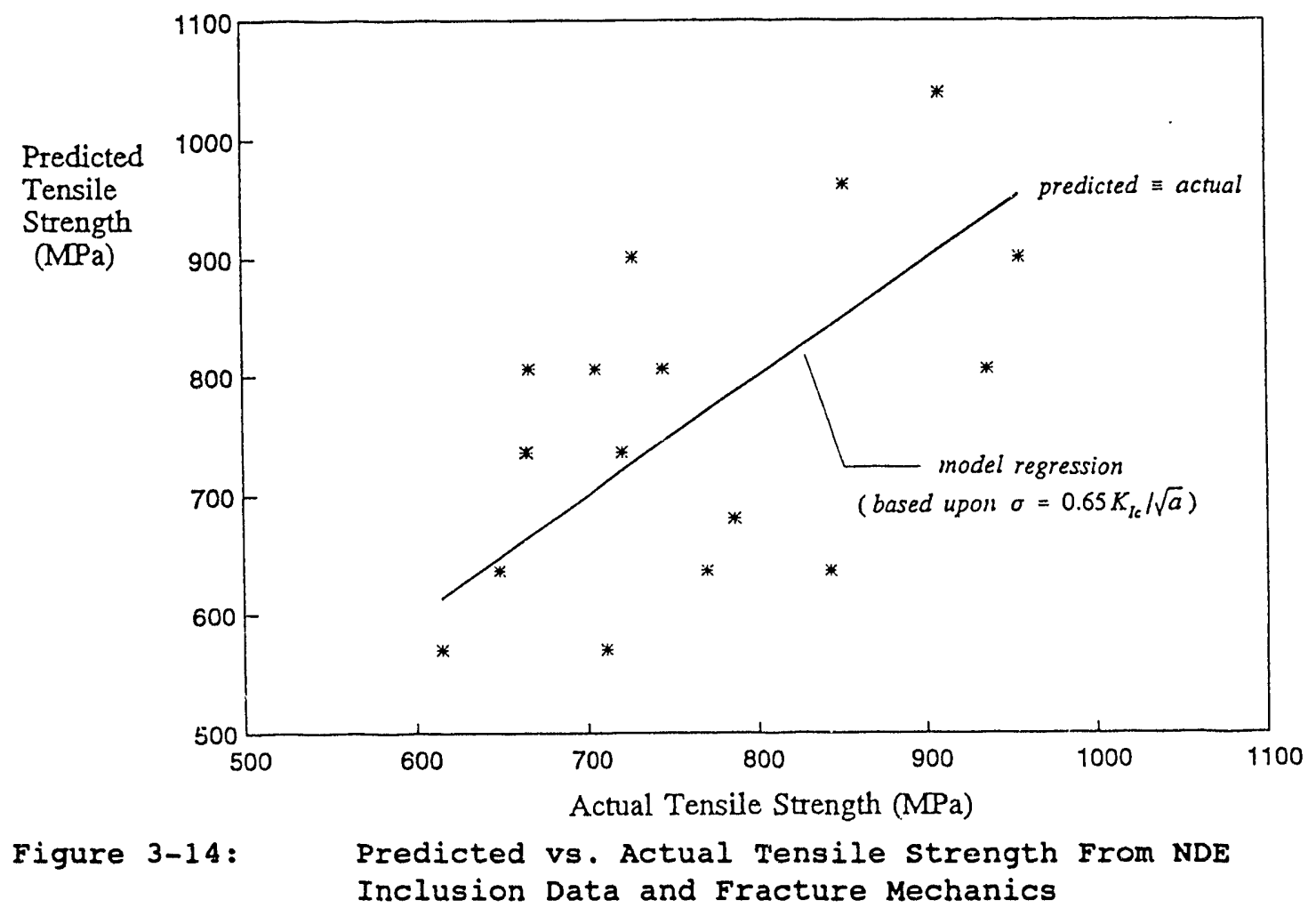

Optimizing X-ray Unit Performance

Several experiments were conducted to establish variations in the intensity of the $x$-ray beam. In order to quantify density gradients in cast specimens, there is a need to know when density variation indications are intrinsic to the sample and not due to scatter or nonuniformity of the $x$-ray jeam. Indirectly, the intensity of the $x$-ray was measured over the width of beam and variations up to 158 were discovered. Two approaches have been pursued to dampen the variation in the beam intensity. $\mathrm{Pb}$ diaphragms were tested and found to reduce the beam intensity difference to 78 , Figure 3-15.

New tungsten targets were designed with target angles less than 
$45^{\circ}$ in order to improve the gray level uniformity. With the shallower targets, the beam intensity difference was reduced to less than 58 with no effect on exposure times as is the case with $\mathrm{Pb}$ diaphragms.

Shadow formations have been observed on magnified $x$-ray film images. The shadows occur on the edge of test objects and are seen as an increase in film density. It has been determined that these shadows are due to secondary radiation produced internally in the tube head. several types of filter material were evaluated for beam hardening and the reduction of internal scatter. One filter was found which absorbed the internal scatter and nearly eliminated the edge burnout on cylindrical samples which vastly improves the detection capabilities. A combination of filters which hardened the beam and eliminated internal scatter was found to increase the edge detection on cylindrical samples by 278. The penalty to this approach is a large increase in exposure time at low energy levels. Another approach was to redesign the tubehead to eliminate the internal scatter. Modifications were made to the tubehead to prevent the formation of secondary radiation and the occurrence of shadows.

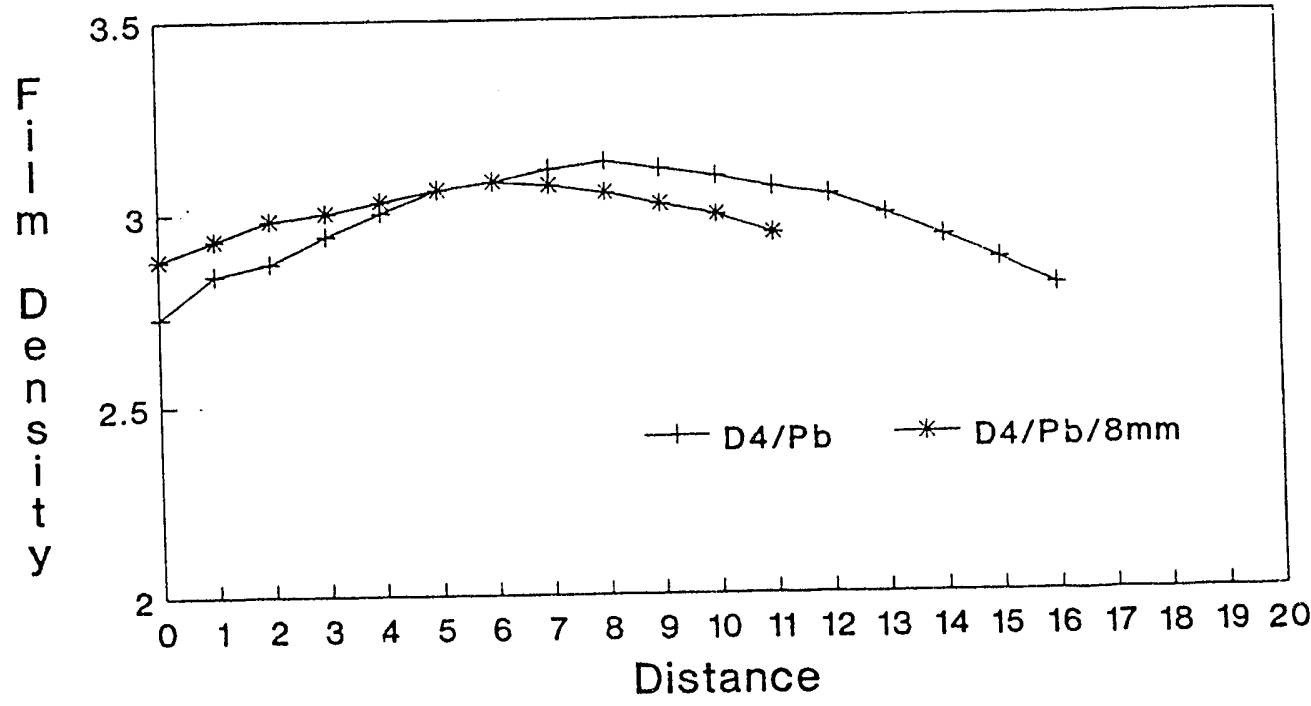

Figure 3-15: Intensity Variations observed in $\mathrm{X}$-ray Beam

\section{Detection of Agglomerates in Dense NSF Bars}

An investigation into the detection sensitivity cf agglomerates in green and HIP'ed NSF bars was conducted. It had been previously established that agglomerates in powder or slip have the radiographic appearance of high density regions. Agglomerates of Si3N4 powder were produced in sizes of 100 to $400 \mu \mathrm{m}$ (using ASTM steel sieves) and samples were taped to the surface of an $11 \mathrm{~mm}$ diameter green bar. The agglomerates were easily detected through the $11 \mathrm{mn}$ diameter. Agglomerates less than $100 \mu \mathrm{m}$ crumbled when being taped, so that the tests were restricted to $>100 \mu \mathrm{m}$.

simulated dense agglomerates were produced by crushing a HIP'ed NCX-5102 billet sample. The pieces were sized from $50-400 \mu \mathrm{m}$ and as 
in the green testing, samples were taped to a dense $6 \mathrm{~mm}$ bar. In this case, agglomerates as small as $65 \mu \mathrm{m}$ were readily detected.

\section{STAGE III PROCESS DEMONSTRATION NDE SUPPORT}

Iiquid Dye Penetrant testing and Microfocus x-ray radiography were utilized as tools to monitor the introduction of defects into the process and for the identification of critical strength limiting flaws during stage III. Microfocus radiography was used as one of the instruments to characterize specimens from all stages of processing for in-process control. It was employed at various points of the process for detection of contaminants in the raw material and colloidal suspensions and for detection of defects in the green and final machined specimens. Iiquid dye penetrant testing was used to identify and measure surface damage on HIP'ed/machined tensile specimens.

The techniques were optimized in stage II for different specimens at each stage of processing. The gage section of green and final machined tensile specimens were radiographed at $10 \mathrm{x}$ magnification in 3 orientations $\left(0^{\circ}, 90^{\circ}\right.$, and $\left.135^{\circ}\right)$ taken from a particular reference point. Low energy $\mathrm{x}$-rays were employed for improved contrast and a thin $\mathrm{Pb}$ filter was used to reduce scatter and edge burnout for the cylindrical specimens. The as-cast specimens were $\mathrm{x}$-rayed typically at $2 \mathrm{x}$ in order to see the entire sample for process evaluation. Different lots of powder and colloidal suspensions were usually examined using 30 grams of sample at $10 x$ magnification. In all cases, a slow speed, fine-grain film was used. A post-emulsifiable, fluorescent penetrant with level IV sensitivity was used for the Liquid Dye Penetrant method. All penetrant samples were examined at 35-350x magnification in the gage area with a fluorescent microscopy system.

\section{In-Process Inspection}

At the initial stages of this iteration, the powder lots (E and $F$ ) which were to he milled during stage III were examined for contamination before hand using microfocus radiography. This was also done for each batch of slip after the closed loop aqueous milling and consolidation steps. No contamination in the form of high density inclusions were observed in either the slip or raw powder.

As stated earlier, microfocus radiography was used as an inprocess control check on the casting operation. On a periodic sampling basis, as-cast NSF green tensile specimens were radiographed immediately upon demolding. The radiographic film for these bars was immediately interpreted and the results fed back to the operations manager for a go/nogo decision. This in-process inspection technique was instrumental in detecting an air leak in the casting system. A sudden rise in radiographic void indications led to the discovery of a leak which was causing air entrapment voids in the cast specimens, Figure 3-16. Corrective actions were completed after a minor interription in the casting operation.

MFX was also used to evaluate the effect of chemical treatment of the plaster of paris molds on the low density region that develops at the filtration surface. The study was pursued as part of the Lot $\mathbf{E}$ 


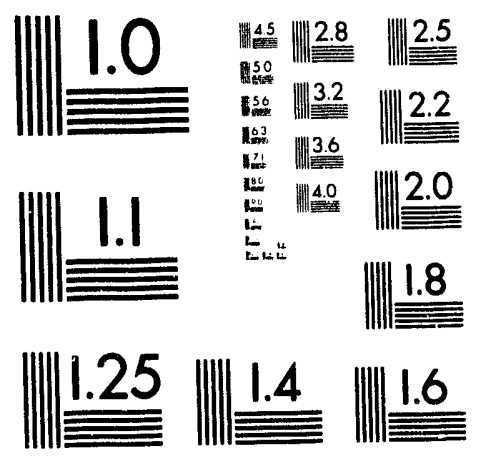



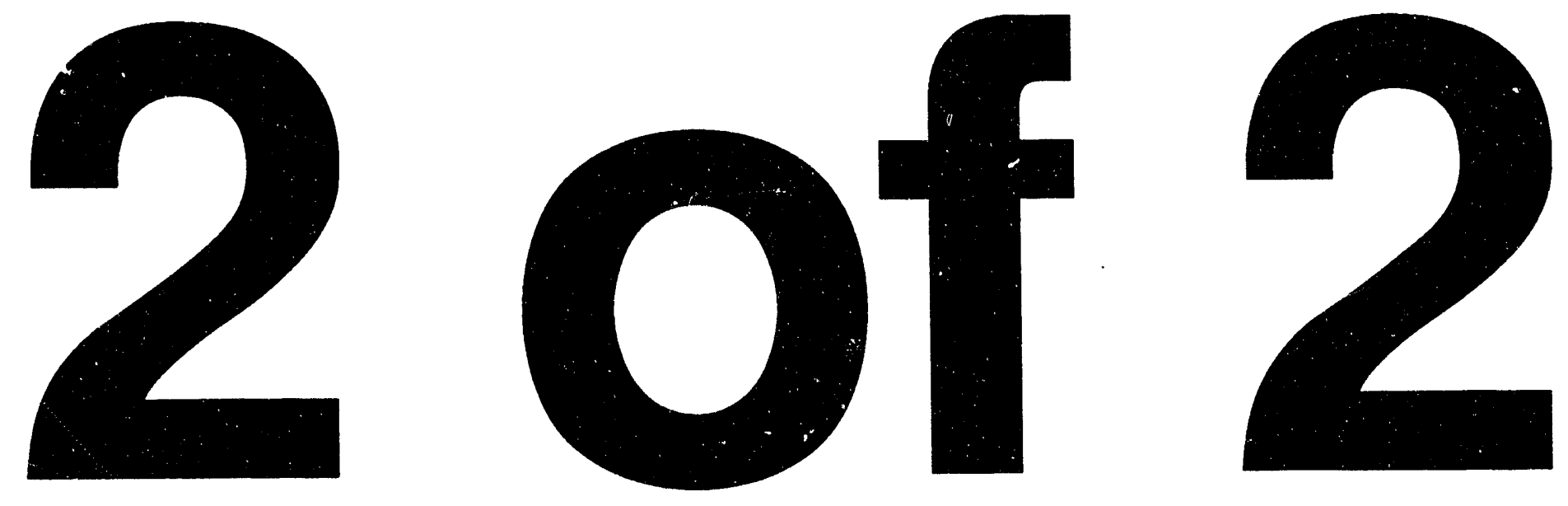
powder evaluation. As mentioned earlier, Lot $\mathbf{E}$ powder behaved differently in colloidal casting behavior and showed a significant shift in the iso-electric point (IEP). Radiography of tensile rod cross sections clearly established that the treatment of the mold with a chemical stabilizer reduces the depth of this low density zone (from 1.2 to $0.3 \mathrm{~mm})$, Figure $3-17$. Cross-sections from four tensile rods cast in molds treated and untreated with a chemical stabilizer were tested in this experiment. The treatment of molds was used with Lot $\mathrm{E}$ powder batches.

Tensile rod cross-sections from two lots of powder (Lot D \& Lot E) were $x$-rayed to see differences in density gradient formation. This would verify if the powders exhibit similar casting behavior. other lots of powder tested behaved very much like Lot $D$ so it was used as the control in the experiment. The low density region associated with the filtration surface was observed in both powders. The depth of the low density zone, as measured on 4 samples from each lot of powder, was substantially reduced on Lot D compared to Lot E, Figure 3-18. These results agreed well with the earlier findings that the Lot $E$ powder was physically and chemically different from earlier lots.
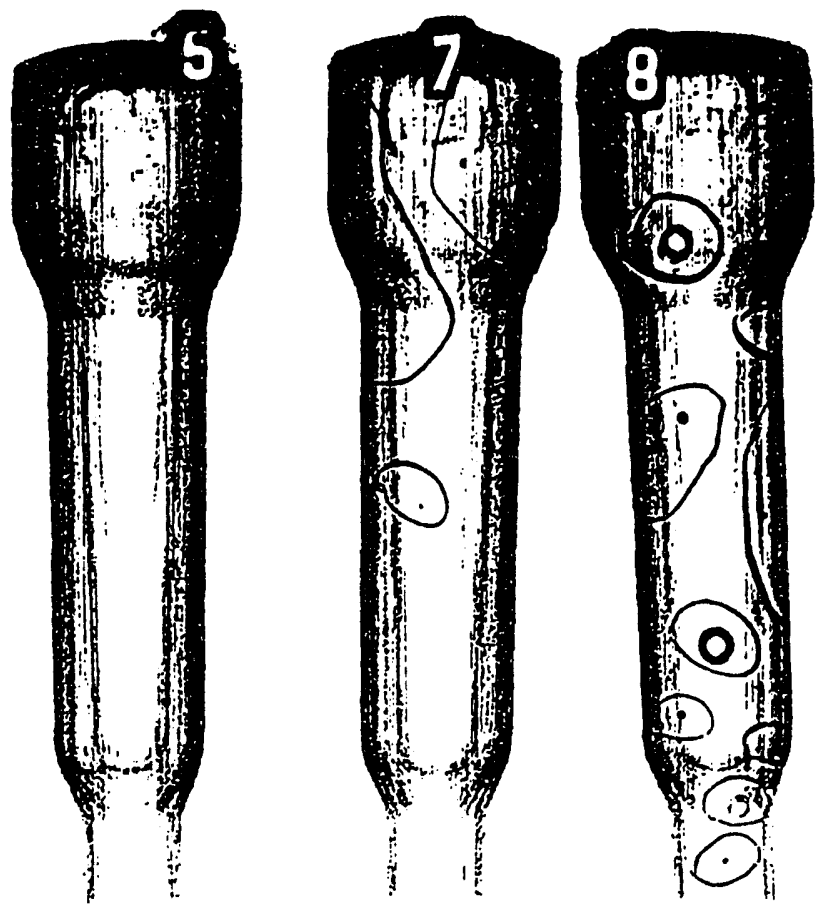

Figure 3-16:

Air-entrapment Voids in Cast NSF Bars Detected Radiographically. 


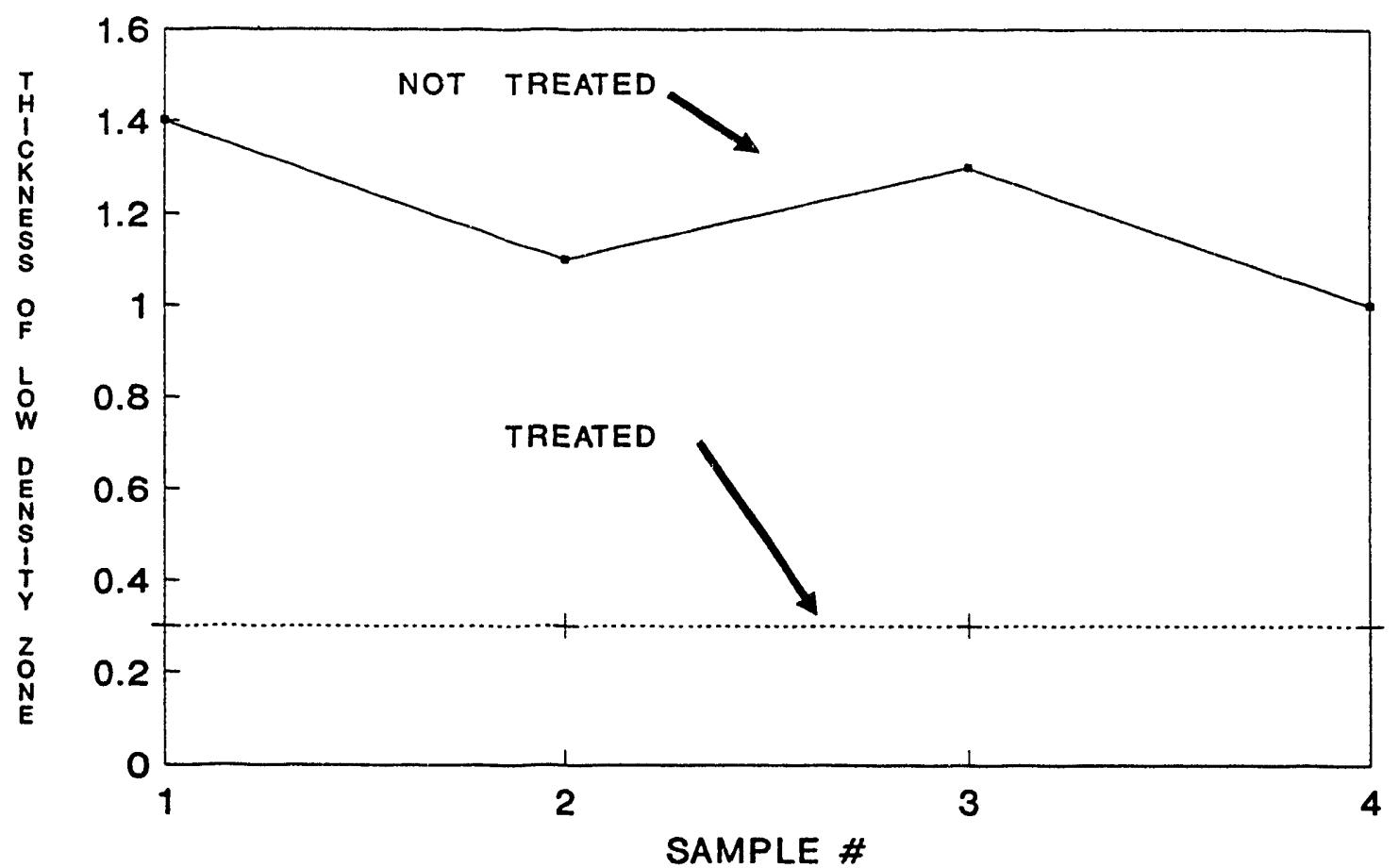

Figure 3-17: MFX Data Illustrating Density Gradient Control Using Mold Treatment

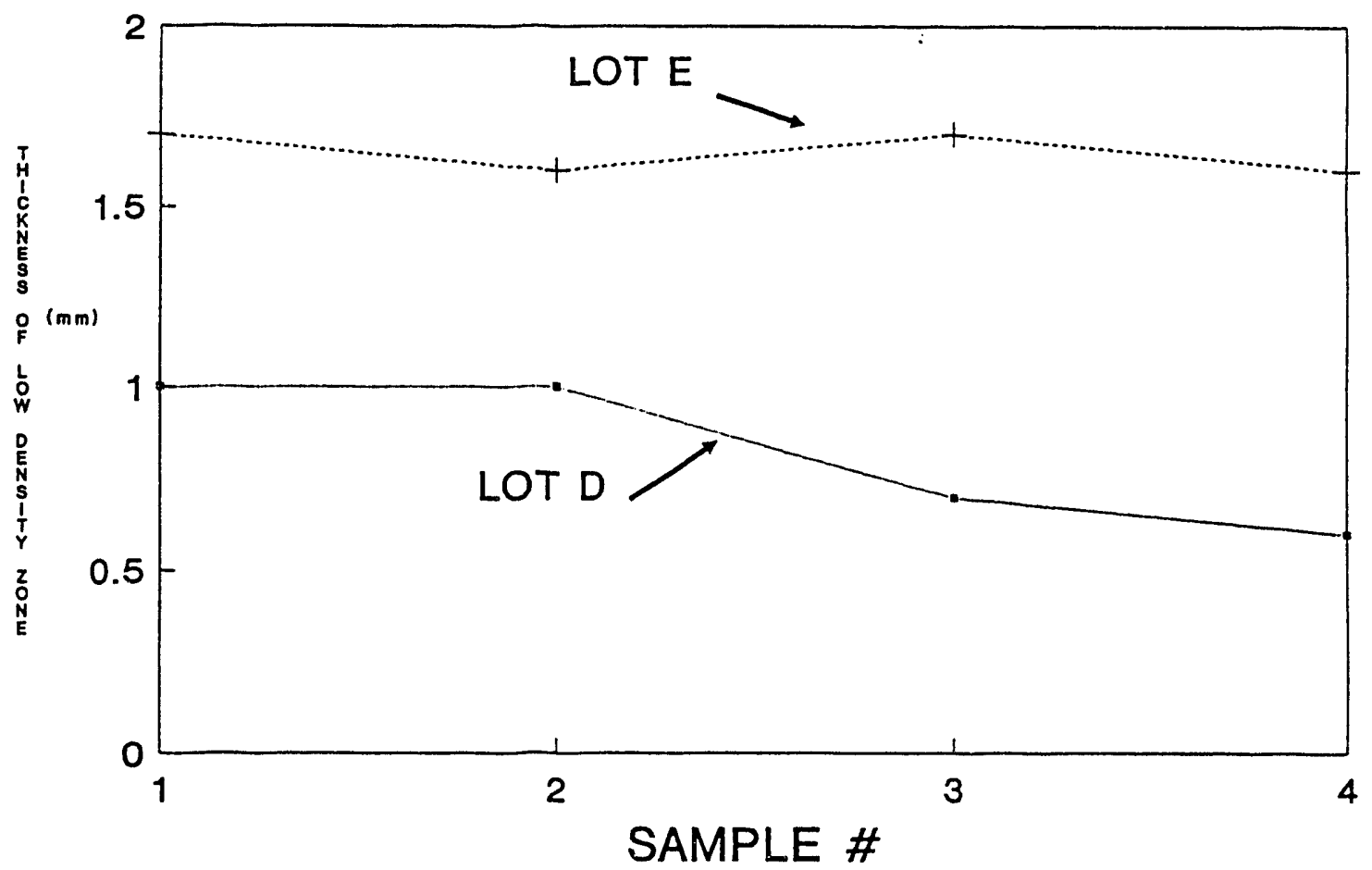

Figure 3-18:

MFX Data Illustrating Powder Lot Variation in Density Gradient. 
Microfocus radiography was used to characterize green tensile specimens at the presinter step of the process. Approximately 48 of the specimens evaluated were removed from the process due to large voids observed in the bulk. These usually occurred in specimens cast at the end of the sequence when there was minimal slip left in the tanks.

\section{Radiography Data for Machined NSF Bars}

All tensile specimens that were HIP'ed/machined were examined by microfocus radiography and Liquid Dye Penetrant testing. A total of 322 final machined test samples were characterized by both techniques. only 17 specimens with high density inclusions, 27 specimens with porosity, and 1 specimen with a radial crack were found with radiography. Figure 3-19 illustrates the size distribution of the 17 inclusions detected by microfocus radiography. The size of the defects was taken as the longest dimension measured normal to the tensile axis. The majority $(10$ of 17) of the inclusions found in the test specimens were in the $0-50 \mu \mathrm{m}$ range. These inclusions had the appearance of glassy metallic zones which were probably formed during the HIP'ing or post-HIP heat treatment processes. The density of the inclusions was radiographically only slightly higher than Si3N4 and significantly lower than Fe and other high atomic number metals.

Only the specimen with the crack was rejected prior to mechanical property testing. It was the intent at the start of this program to test all specimens for mechanical properties data unless it was absolutely obvious the sample was rejectable. mhe three specimens with high density inclusions $>150 \mu \mathrm{m}$ had tensile strengths of 930-1080 MPa. Post failure analysis indicaced that the fracture origin of the lowest strength test sample had a $\mathrm{Ti}$ inclusion $(40 \mu \mathrm{m} \times 30 \mu \mathrm{m})$, whereas the other two samples appeared to fail from surface amorphous regions. From standard fracture mechanics, the predicted strength for a $150 \mu \mathrm{m}$ inclusion would be $-600 \mathrm{MPa}$. In Figure $3-20$, the size distribution of the porosity is shown. Over 938 of the porosity observed with microfocus $x$-ray was between 40-100 $\mu \mathrm{m}$ and at the surface.

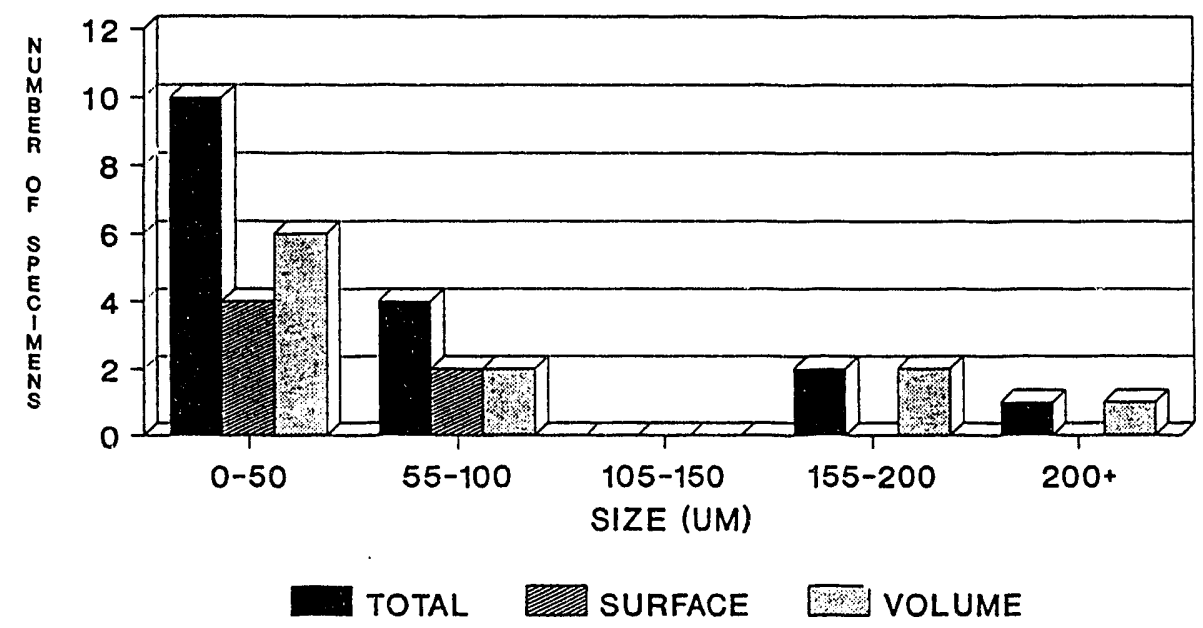

Figure 3-19: Inclusions Detected by MFX in HIP'ed/Machined stage III Tensile Rods 


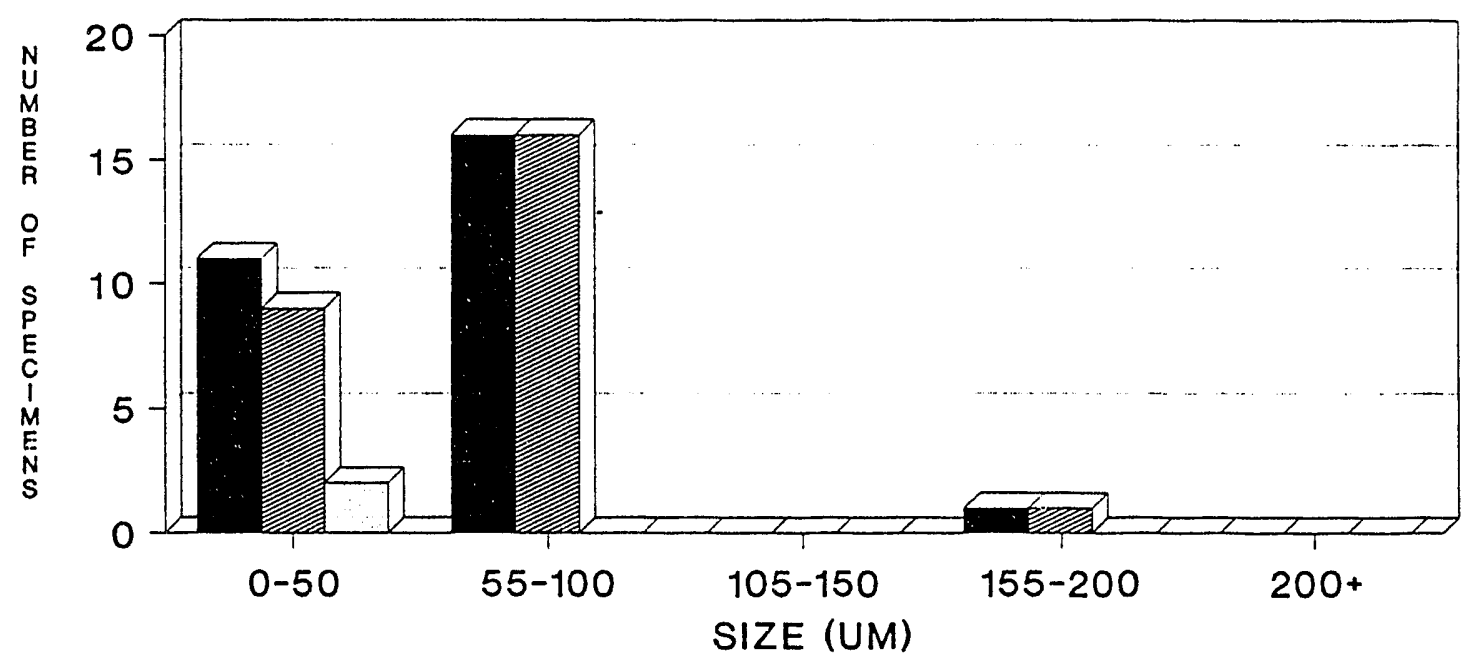

$\begin{array}{ll}\text { TOTAL } & \text { TOE SURFACE } \\ \text { Figure 3-20: } & \begin{array}{l}\text { Pores Detected by MFX in HIP'ed/Machined stage III } \\ \text { Tensile Rods }\end{array}\end{array}$

\section{Liquid Dye Penetrant Data for Machined NSF Bars}

As mentioned, the surface of all HIP'ed/machined tensile rods were characterized by liquid dye penetrant testing (IDP). The four major types of flaws observed with this technigue were cracks (machining related), machining grooves, porosity, and low density areas. Nearly 388 of the specimens were found to have machining related cracks. The cracks observed were radial cracks that extended from the machining grooves. The relative frequency and size distribution of the cracks are depicted in Figure 3-21. The average size of a crack measured from the edge of the machining groove was $12 \mu \mathrm{m}$ (see Figure 3-22). The two major flaw populations observed with IDP were surface porosity and machining damage (grooves). The porosity was present in small clusters with an average size of $20 \mu \mathrm{m}$. These pore clusters were made up of 1-2 $\mu \mathrm{m}$ size pores which were indistinguishable due to blotout during the IDP process. The size distribution of the porosity is seen in Figure $i-23$. Micrographs of typical pore clusters are illustrated in Figure 3-2 $\hat{2}$. The other major flaw type, grinding grooves, occurred in nearly 908 of the specimens. These grooves from dense machining ran parallel to the tensile direction. In many instances, either surface or subsurface cracks were associated with the grinding grooves. Figure 3-25 shows micrographs of typical machining grooves detected by LDP. The width of a machining groove was generally about $5 \mu \mathrm{m}$. Figure 3-26 shows images of grinding grooves detected with ultrasonics using a surface wave 100 MHz transducer. The width of the grinding grooves in the ultrasonic images is 75-80 $\mathrm{mm}$. The resolution of the surface wave transducer is 70 $\mu \mathrm{m}$ suggesting that the grooves seen with ultrasonics are a compilation of grooves that are spaced less than $70 \mu \mathrm{m}$ apart. 


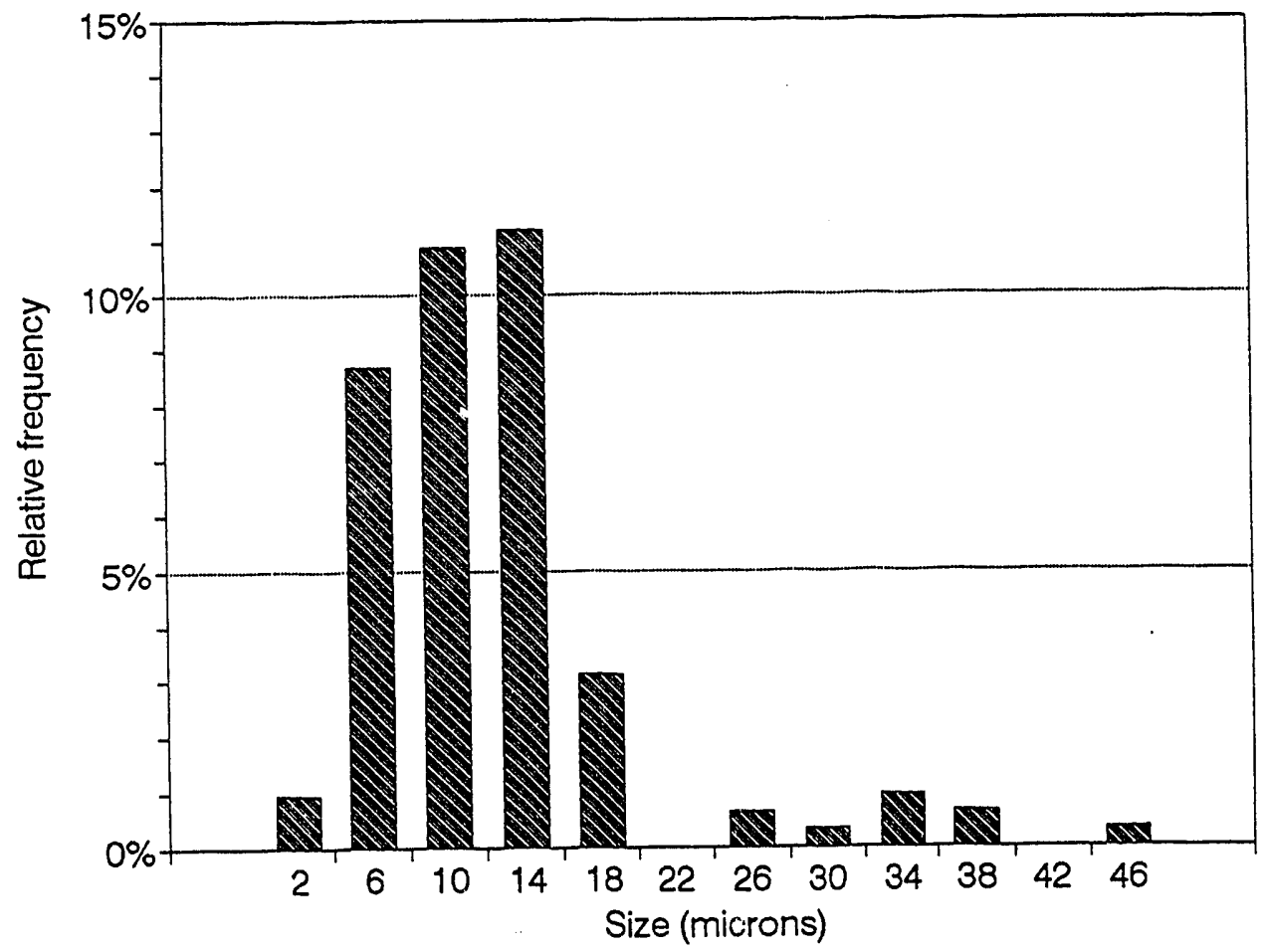

Figure 3-21: Machining Crack Data Detected by IDP in HIP'ed/Machined Stage III Tensile Rods

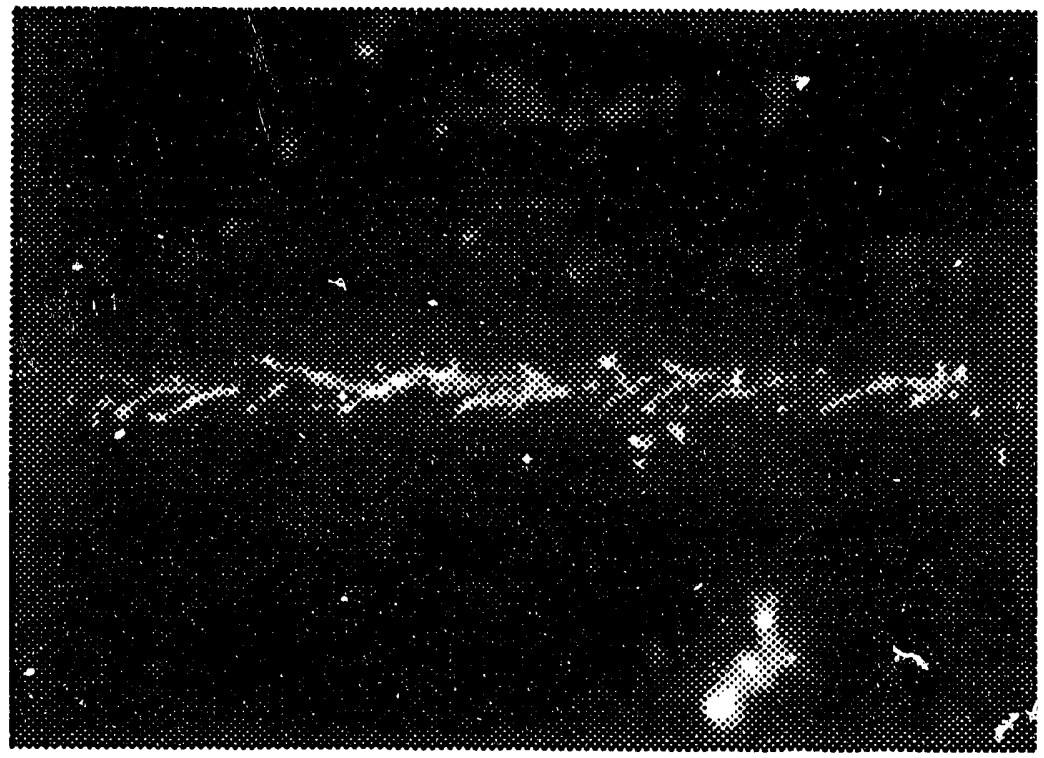

Figure 3-22:

A $6 \mu \mathrm{m}$ wide Grinding Groove with 8-35 $\mu \mathrm{m}$ Radial Cracks (750X) 


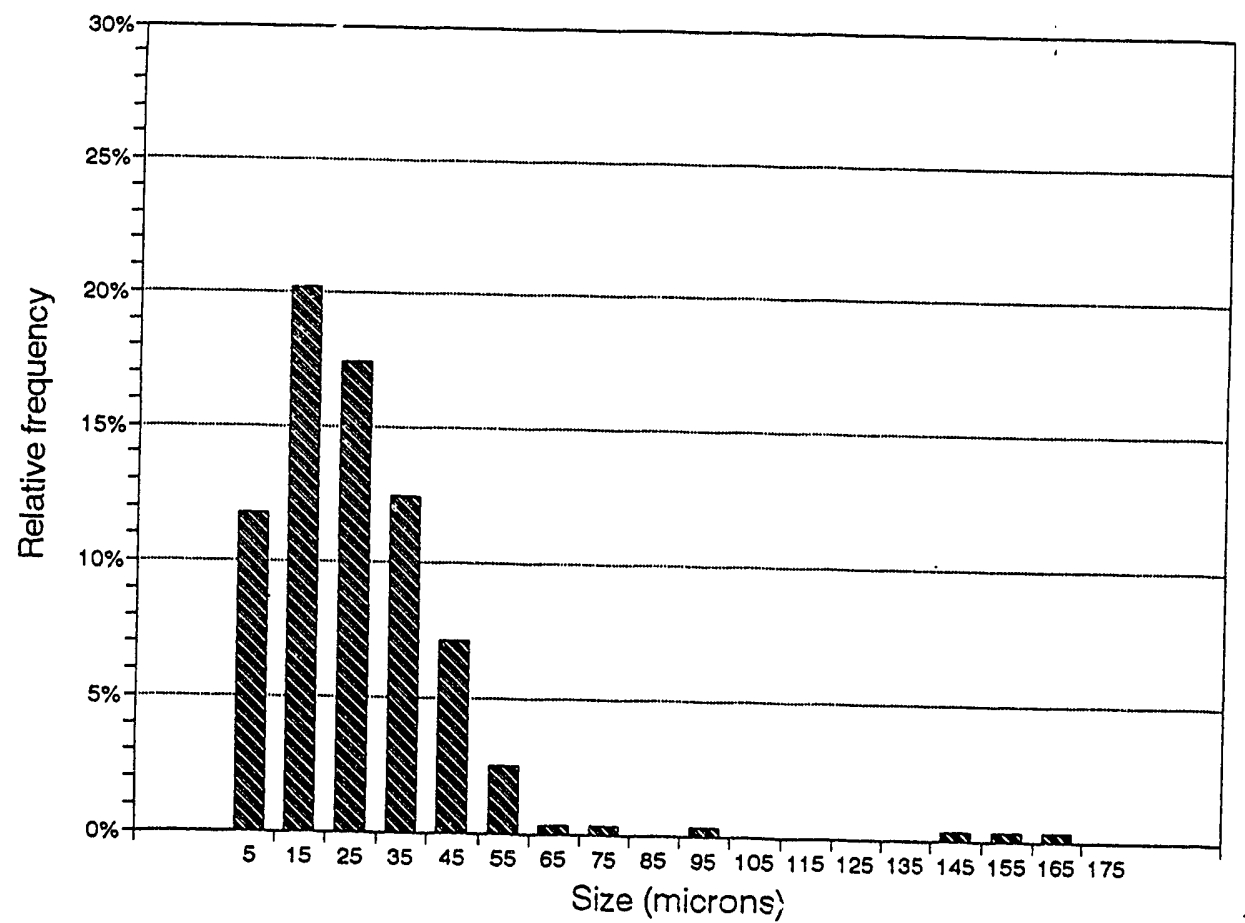

Figure 3-23: $\quad$ Surface Porosity of Machined stage III Tensile Rods by IDP

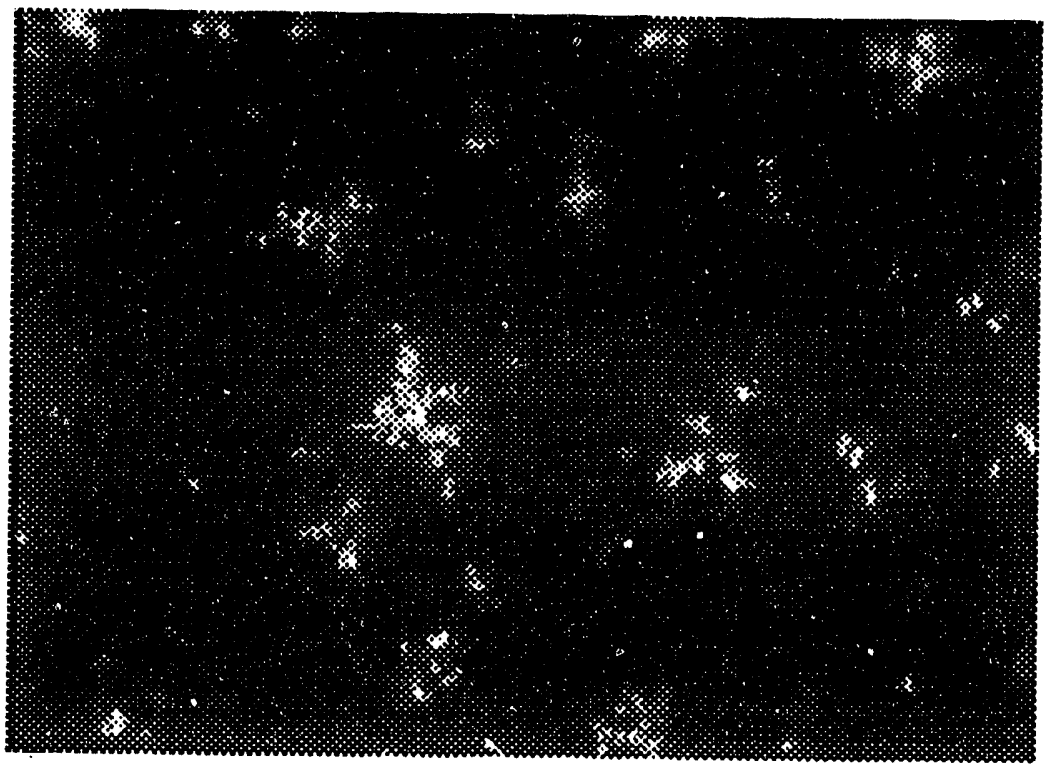

Figure 3-24: Pore Clusters $(13-16 \mu \mathrm{m})$ on Machined surface of stage III Tensile Rods 


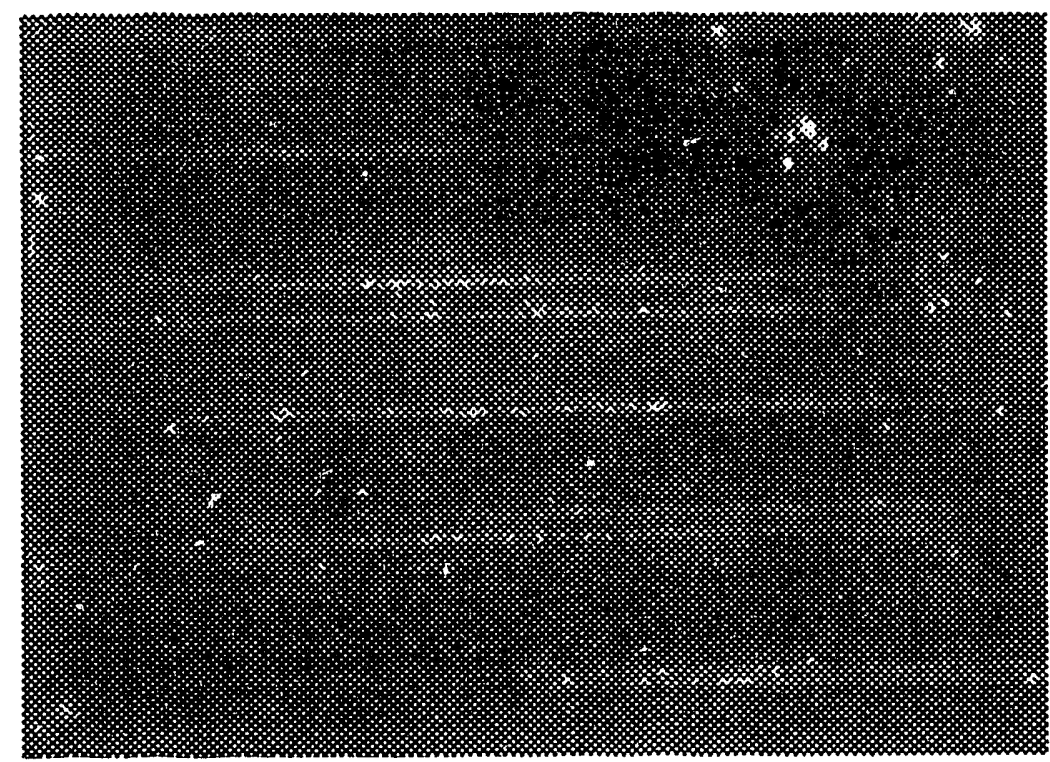

Figure 3-25:

Grinding Grooves ( $5 \mu \mathrm{m}$ wide) Detected by LDP

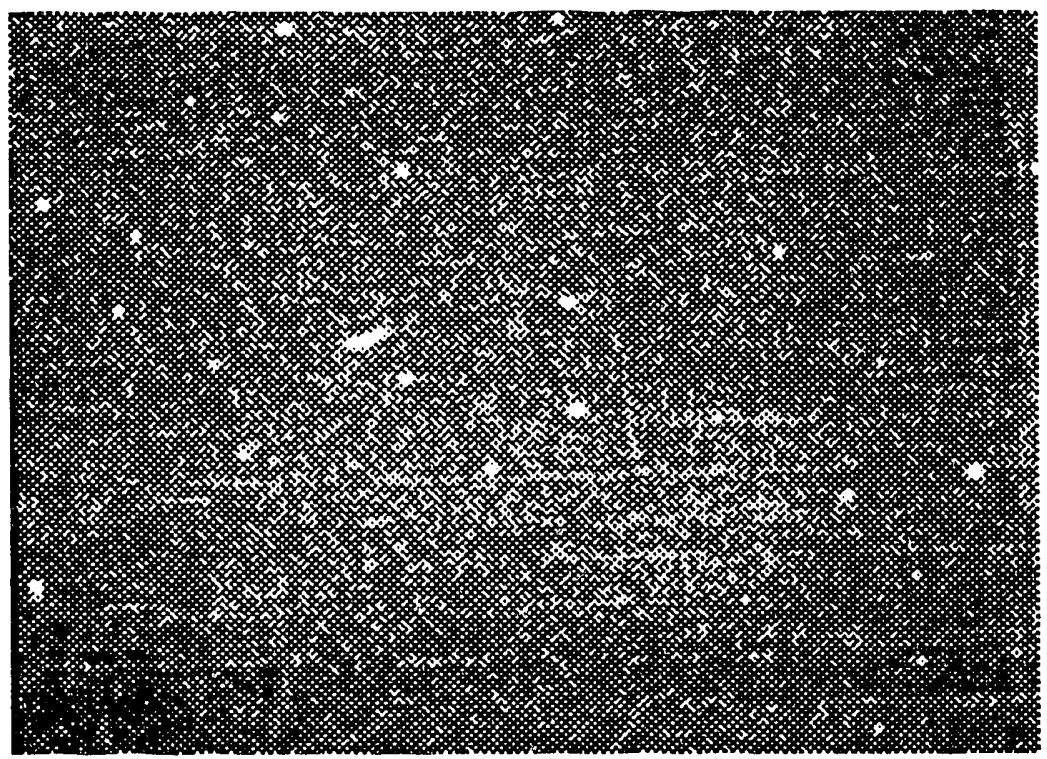

Figure 3-26:

Ultrasonic C-scan of NSF Machined Surface Indicating $70 \mu \mathrm{m}$ wide Grinding Grooves 


\section{REFERENCES}

1. V.K. Pujari, L.C. Sales, J.S. Wang and J. Fraser, "Precision Casting Process Control by Ultrasonic Technique," Ceramic Engineering and Science Proceedings, 13, No. 7-8, 1992, 536-545.

2. S.I. Dieckman et.al., "Three-Dimensional Nuclear Magnetic Resonance Imaging of Green-State Ceramics, "Argonne National Laboratory Report ANL-91/39, May, 1991.

3. N. Gopalsami, P. Rizo, W.A. Ellingson and D.M. Tracey, "Detection Sensitivity of X-ray CT Imaging for NDE of Green-State Ceramics," Review of Progress in Quantitative NDE, 11, D.O. Thompson and D.E. Chimenti, Editors, Plenum Press, N.Y., 1992, 1861-1868.

4. D.J. Snoha and M.R. Foley, "An Investigation of Residual stresses in Machined Silicon Nitride, "Army Materials Technology Laboratory TR 92-46, July 1992 . 


\section{APPENDIX 4: PROPERTY TESTING AND MICROSTRUCTURAL EVALUATION}

The near-net shape formed (NSF) buttonhead tensile rod was the component used throughout the program to evaluate process iterations. Tensile testing was the primary evaluation method. Tensile rods were used to establish representative fast fracture strength distributions at room temperature and at $1370^{\circ} \mathrm{C}$. Stress rupture and creep behavior were also tested using the buttonhead specimen. Fracture toughness tests were performed using $3 \times 4 \times 50 \mathrm{~mm}$ flexure bars machined either from tensile rods or from tile produced within the process iteration.

The primary purpose of the extensive tensile testing conducted in the program was to establish a comprehensive understanding of how flaw populations and process parameters influence mechanical properties. Hence, a thorough inspection strategy was followed at each step of the process to form the necessary database to establish correlations. For instance, prior to machining of the specimens density and runout measurements were conducted. After machining, dimensional tolerance and surface finish were evaluated. Also at this step, radiographic and liquid dye penetrant evaluations were conducted. The entire flow diagram for the tensile specimens from densification to post-mortem analysis is shown in Figure $4-1$.

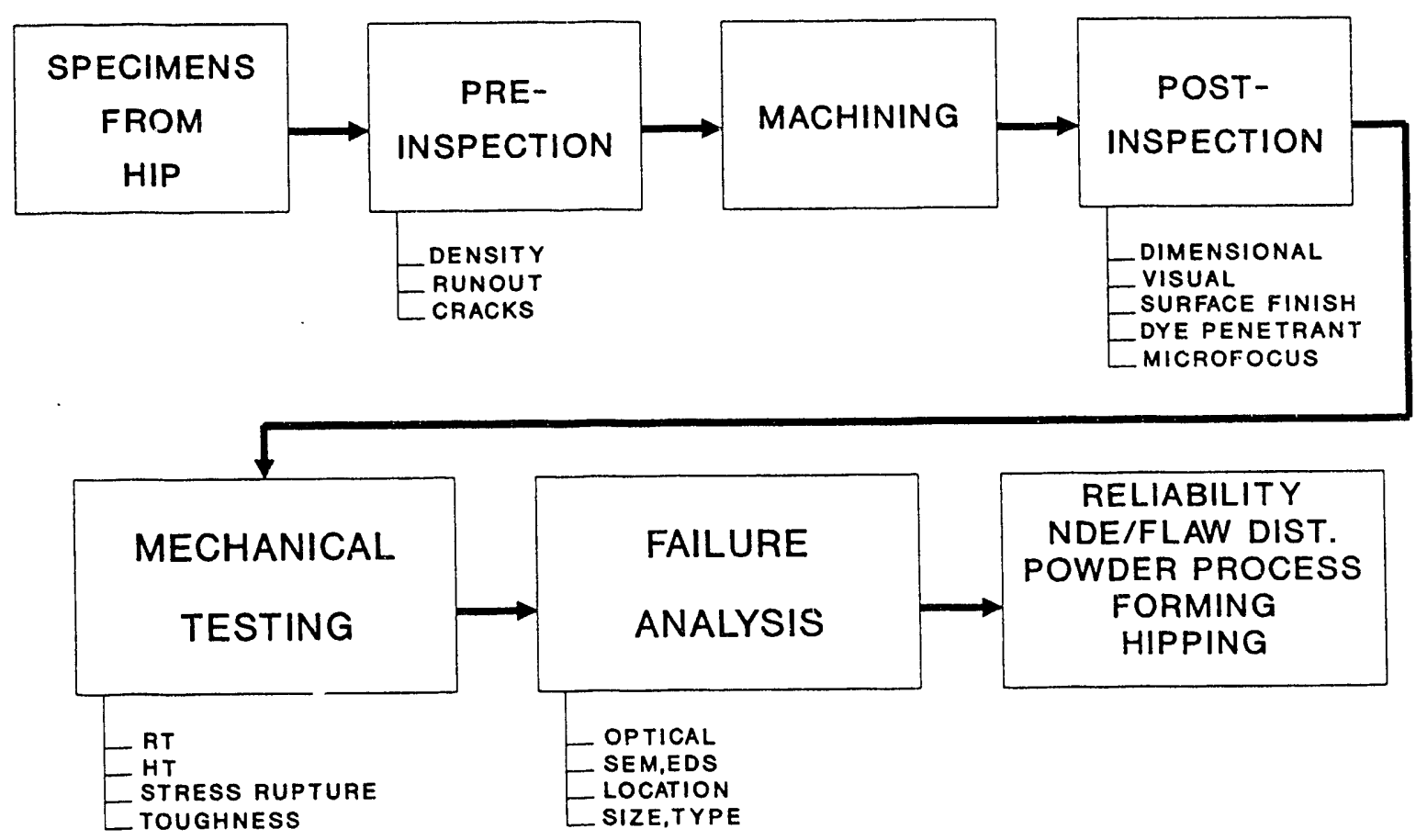

Figure 4-1: Specimen Flow Diagram for Testing and Failure Analysis. 


\section{TESTING PROCEDURES}

\section{Dense Tensile Testing}

The cylindrical buttonhead tensile specimens ${ }^{1}$ were tested on an Instron Model 8562 utilizing the Instron "Super-grip" and straight trisplit copper collet system. A double ramp loading procedure was used to test all specimens. The specimen is initially loaded to $6668 \mathrm{~N}$ (1500 lbs.) at $39 \mathrm{MPa} / \mathrm{min}(250 \mathrm{lbs} . / \mathrm{min})$. This allows time for the fully annealed copper collets to deform to the buttonhead radius of the specimen. After the initial ramp to $6668 \mathrm{~N}$, the specimen is then loaded to failure at a stressing rate of $600 \mathrm{MPa} / \mathrm{min}$.

The load train is checked before testing with an alignment tool for actuator/load cell alignment and a strain gaged tensile specimen for Instron "Supergrip" alignment. Each specimen was marked on the surface (outside the gage section) relative to its position in the tensile grips to allow location of surface failures relative to the grips, as an additional check on alignment. The diameter of the specimen was measured after failure near the fracture surface to eliminate the possibility of surface damage from the calipers prior to testing. The micro-location of the failure origin is determined (gage vs. transition) by the measurement.

Optical fractography was performed on all specimens. Following optical observations, all specimens were submitted for scanning electron microscopy, SEM, and energy dispersive spectroscopy, EDS. Low and high magnification micrographs were taken of each fracture surface and flaw origin. The EDS is performed at each origin to determine if any impurities are present. The location, type and size of each flaw were noted, measured and tabulated for database evaluation.

Procedures for tensile testing at room temperature and elevated temperature were established by testing NT154 silicon nitride cylindrical buttonhead specimens at three gage diameters. The RT and $1370^{\circ} \mathrm{C}$ strength data are summarized in Rable 4-1. Optical fractography of the RT specimens indicated 35 of 37 specimens failed at the surface while the $1370^{\circ} \mathrm{C}$ specimens all failed from bulk flaws. Also approximately 208 of the RT specimens failed at the gage transition region where finite element modelling has indicated a slight stress elevation.

Table 4-1: Tensile Data from NT154 Samples

\begin{tabular}{||c|c|c|c|c|c||}
\hline \multirow{2}{*}{$\begin{array}{c}\text { Gage } \\
\text { Diameter } \\
(\mathrm{mm})\end{array}$} & \multicolumn{3}{|c|}{$20^{\circ} \mathrm{C}$} & \multicolumn{2}{c|}{$1370^{\circ} \mathrm{C}$} \\
\cline { 2 - 6 } & $\begin{array}{c}\text { Mean } \\
\text { Strength } \\
(\mathrm{MPa})\end{array}$ & $\begin{array}{c}\text { Standard } \\
\text { Deviation } \\
(\text { MPa }\end{array}$ & Number & $\begin{array}{c}\text { Mean } \\
\text { Strength } \\
\text { (MPa) }\end{array}$ & Number \\
\hline \hline 4.5 & 716 & 83 & 12 & 431 & 2 \\
\hline 5.5 & 685 & 76 & 12 & 428 & 2 \\
\hline 6.35 & 711 & 77 & 13 & 449 & 3 \\
\hline
\end{tabular}


Green Tensile Testing

Fixtures were designed and an sop developed for testing green and presintered NSF tensile bars. As-injection molded specimens were found to have mean strength at the $3.0 \mathrm{MPa}$ level. The variability was found to depend strongly upon the processing conditions.

Tests were conducted on pre-sintered NSF bars processed from both the injection molding and pressure casting routes. Strengths were found to fall in the $5.0-8.0 \mathrm{MPa}$ range for each case.

The molded specimen failure origins included agglomerates, dewaxing cracks and metallic inclusions. The cast specimen failure origins consisted of agglomerates, pores and hoop cracks formed during drying.

\section{BASELINE TENSILE DATA}

\section{Injection Molding}

The first iteration used injection molding to form NSF tensile specimens. A total of 49 specimens were tested for RT fast fracture strength and of these, 26 failed from gage section defects, while the remainder failed from buttonhead defects. The strength data are summarized in Table 4-2. The strength for the specimens which failed at the buttonhead was calculated as the stress in the gage section at the point of buttonhead failure. Also included in Table 4-2 are data for 35 flexure specimens machined from five tensile rods and tested at room temperature.

Table 4-2: Injection Molding Baseline RT Tensile Strength Data

\begin{tabular}{||l|c|c|c|c|}
\hline \multicolumn{1}{|c|}{$\begin{array}{c}\text { Failure } \\
\text { Location }\end{array}$} & $\begin{array}{c}\text { Average } \\
\text { Strength } \\
\text { (MPa) }\end{array}$ & $\begin{array}{c}\text { Standard } \\
\text { Deviation }\end{array}$ & Range & n \\
\hline \hline Gage/Volume & 506 & 91 & $327-663$ & 17 \\
\hline Gage/Surface & 356 & 201 & $38-629$ & 9 \\
\hline Buttonhead & 442 & 94 & $276-632$ & 23 \\
\hline All & 454 & 121 & $38-663$ & 49 \\
\hline Flexure & 760 & 159 & $255-965$ & 35 \\
\hline
\end{tabular}

The mean strength for al.1 49 specimens was $454 \mathrm{MPa}$ with a characteristic strength of $492 \mathrm{MPa}$ and Weibull Modulus of 4.2. The strength data compares well with early PY6 injection molded data ${ }^{2}$ and is slightly lower than more recent data.

Fractography established that all 23 buttonhead failures were from pre-existing surface cracls due to the injection molding process. These flaws were detected using a dye penetrant technique as part of the post- 
unachining inspection. Of the 26 gage failures, 17 failed from volume defects and 9 from surface defects. The defects were mainly inclusions with a few agglomerates and pores. The inclusions were either Fe, Ti, $A l, C r, 2 r, N i$ or a combination of these. The source of the defects was traced to the binders used as well as the injection molding equipment. The size of the flaws ranged from 100 to $500 \mu \mathrm{m}$.

Fracture toughness mecsurements ${ }^{4}$ performed on flexure bars

machined from tensile rods resulted in an average $R_{x c}$ value of $5.35 \pm$ $0.037 \mathrm{MPa} \sqrt{\mathrm{m}}$.

Fast fracture tensile testing was performed on 15 specimens at $1370^{\circ} \mathrm{C}$ in air and the data are summarized in Table 4-3.

Table 4-3: Injection Molding Baseline $1370^{\circ} \mathrm{C}$ Tensile strength Data

\begin{tabular}{|l|c|c|c|c|}
\hline \multicolumn{1}{|c|}{$\begin{array}{c}\text { Failure } \\
\text { Location }\end{array}$} & $\begin{array}{c}\text { Average } \\
\text { Strength } \\
\text { (MPa) }\end{array}$ & $\begin{array}{c}\text { Standard } \\
\text { Deviation }\end{array}$ & Range & n \\
\hline \hline Gage & 199 & 127 & $64-370$ & 13 \\
\hline Buttonhead & 236 & -- & $230-242$ & 2 \\
\hline All & 204 & 118 & $64-370$ & 15 \\
\hline Flexure & 456 & 18 & $150-607$ & 14 \\
\hline
\end{tabular}

There were only two buttonhead failures caused by pre-existing surface cracks which were introduced during forming. Seven of the remaining 13 failed due to slow crack growth at stresses below $140 \mathrm{MPa}$, while the other six failed at stress levels in excess of $250 \mathrm{MPa}$. Also included in Table 4-3 are data for 14 flexure specimens machined from five tensile rods. As expected, the strengths are greater in flexure than in tension.

Tensile stress rupture tests were performed on five specimens at $1230^{\circ} \mathrm{C}$ in air at an applied stress level of $350 \mathrm{MPa}$. The times to failure were $0,2,5$, and 20 minutes, with one specimen failing during loading at a gage stress of $278 \mathrm{MPa}$. All specimens failed in a fast fracture mode as opposed to slow crack growth. This may be explained by considering the fact that the average strengths at room temperature and $1370^{\circ} \mathrm{C}$ were 454 and $204 \mathrm{MPa}$, respectively. These data suggest that the applied stress of $350 \mathrm{MPa}$ is very close to the fast fracture strength of this material at $1230^{\circ} \mathrm{C}$. Flexure stress rupture tests were also performed on four specimens machined from tensile rods. One failed immediately and three survived 200 hours before test termination.

\section{Colloidal Consolidation}

Room temperature fast fracture strength tests were performed on 28 specimens from the baseline pressure casting iteration. In this group of specimens, eight were HIP'ed to less than 998 theoretical density. Figure 4-2 illustrates that strength falls off significantly below 998 
T.D. and for that reason the 8 low density specimens were not used in the iteration data analysis.

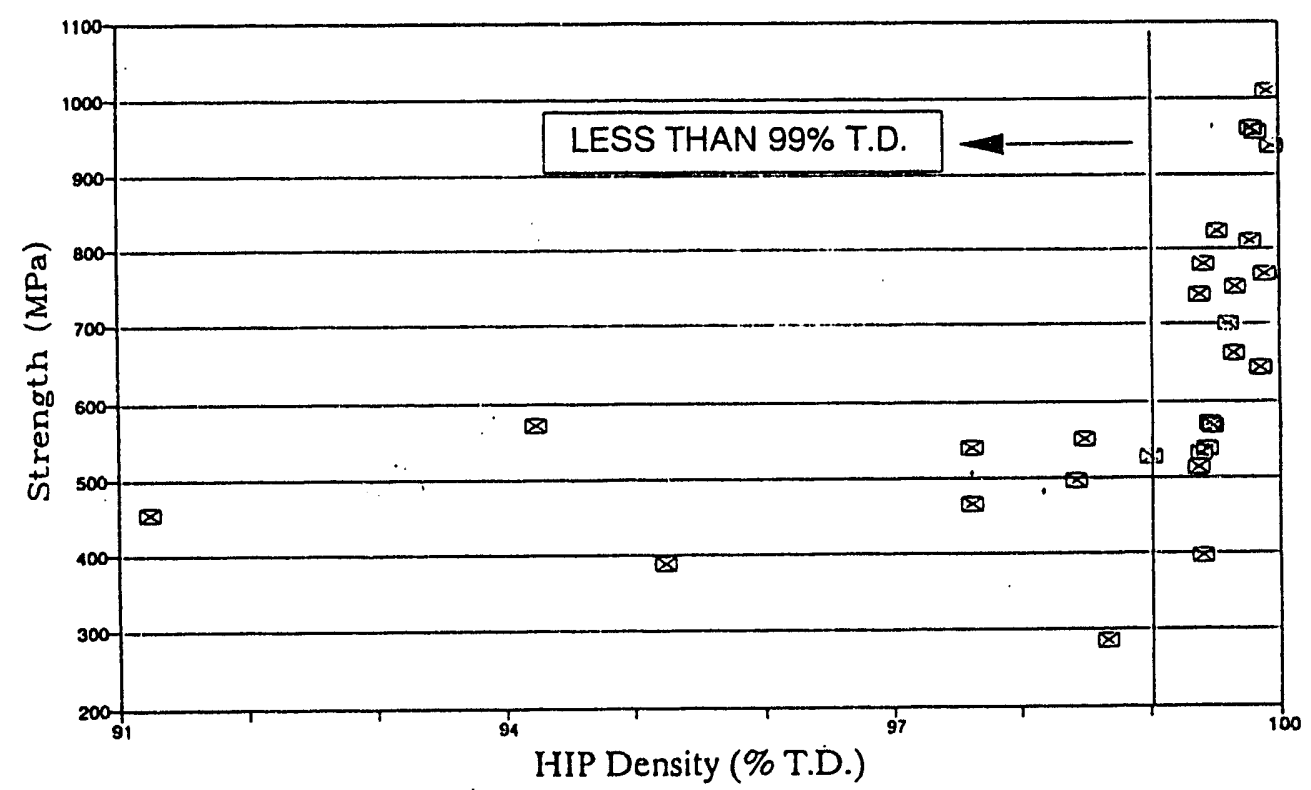

Figure 4-2: Strength vs. Density for Pressure Cast Tensile Rods

The tensile strength data for the 20 valid specimens are summarized in Table 4-4. There were 9 volume, 6 surface, 4 buttonhead and one unknown failure origins. The overall average tensile strength and Weibull Modulus are $709 \mathrm{MPa}$ and 4.7, respectively.

Table 4-4: Pressure Cast Baseline RT Tensile Strength Data

\begin{tabular}{|l|c|c|c|c||}
\hline \multicolumn{1}{|c|}{$\begin{array}{c}\text { Failure } \\
\text { Location }\end{array}$} & $\begin{array}{c}\text { Mean } \\
\text { (MPa) }\end{array}$ & $\begin{array}{c}\text { Standard } \\
\text { Deviation } \\
\text { (MPa) }\end{array}$ & $\begin{array}{c}\text { Range } \\
\text { (MPa) }\end{array}$ & $\begin{array}{c}\text { Number of } \\
\text { Specimens }\end{array}$ \\
\hline Gage/volume & 636 & 193 & $397-1010$ & 9 \\
\hline Gage/surface & 845 & 118 & $701-961$ & 6 \\
\hline Buttonhead & 628 & 121 & $532-810$ & 4 \\
\hline All & 709 & 174 & $397-1010$ & $20 *$ \\
\hline Flexure & 857 & 126 & $527-1177$ & 60 \\
\hline
\end{tabular}

* i.jeludes one unknown flaw origin

The tensile specimens included in the above data set were cast from two different slips (C-series and $W$-series) as part of a larger 
matrix of experiments. Table 4-5 sumarizes the strength data according to slip type. The bars cast from C-series slip show a much higher tensile strength ( $862 \mathrm{MPa}$ ) and Weibull Modulus (10.0) than for bars cast from w-series slip.

Table 4-5: Slip Comparison for Pressure Cast silicon Nitride

\begin{tabular}{||c|c|c|c|c|c||}
\hline Slip & $\begin{array}{c}\text { Mean } \\
\text { Strength } \\
\text { (MPa) }\end{array}$ & $\begin{array}{c}\text { Standard } \\
\text { Deviation } \\
\text { (MPa) }\end{array}$ & $\begin{array}{c}\text { Characteristic } \\
\text { Strength } \\
\text { (MPa) }\end{array}$ & $\begin{array}{c}\text { Weibull } \\
\text { Mociulus } \\
\text { m }\end{array}$ & $\mathbf{n}$ \\
\hline \hline C Series & 862 & 136 & 911 & 10.1 & 5 \\
\hline w Series & 658 & 158 & 719 & 4.6 & 15 \\
\hline
\end{tabular}

Fractographic analysis found that the major flaw population in this iteration was that of agglomerates. In one case, a silicon inclusion was detected within an agglomerate failure origin. In all other instances the chemistry of the failure origin was identical to the matrix as determined by EDS in the SEM (Figure 4-3). The agglomerates were found both in the volume and at the surface. It should be noted that the specimens that failed at the surface had machining damage concurrent with an agglomerate. The size range of the agglomerates at the fracture origin for the NSF bars made from C- and W-series slips were measured to be 10-25 $\mu \mathrm{m}$ and $25-100 \mu \mathrm{m}$, respectively, much smaller than the 100-500 $\mu \mathrm{m}$ sized defects identified as failure origins of the injection molded baseline specimens. The improved pressure cast strength data reflects the reduction in flaw size.

Fracture toughness was measured by the indentation strength (IS) technique on MOR bars machined from NSF tensile rods. The values ranged from 4.5 to $6.2 \mathrm{MPa} / \mathrm{m}$. Powder XRD was performed on the specimens with the lowest and highest toughness values to determine the amount of retained $\alpha$ crystal structure. The specimen with the toughness of 4.5 MPa $\sqrt{m}$ had $648 \alpha$ phase as compared to only $188 \alpha$ phase for those specimens which measured $\mathrm{Kic}$ of $6.2 \mathrm{MPa} / \mathrm{m}$. The toughness value measured by the indentation fracture (not IS) technique is plotted in Figure 4-4 as a function of $8 \alpha$ phase silicon nitride for each of the iteration tensile specimens. As expected, a definite trend of decreasing fracture toughness with increasing retained $\alpha$ phase is apparent from the plot. These results provided strong evidence that the goal of improving reliability would require both microstructure (Ric) and defect control.

Fast fracture tensile tests were performed on four specimens at $1370^{\circ} \mathrm{C}$ in air with a resulting average strength and standard deviation of $361 \pm 3.5 \mathrm{MPa}$. Optical fractography indicated two volume and two surface flaw origins consisting of agglomerates (same composition as surrounding matrix). High temperature fast fracture testing was also performed on 20 flexure specimens machined from tensile rods, with a resulting average strength of $575 \pm 52 \mathrm{MPa}$. 

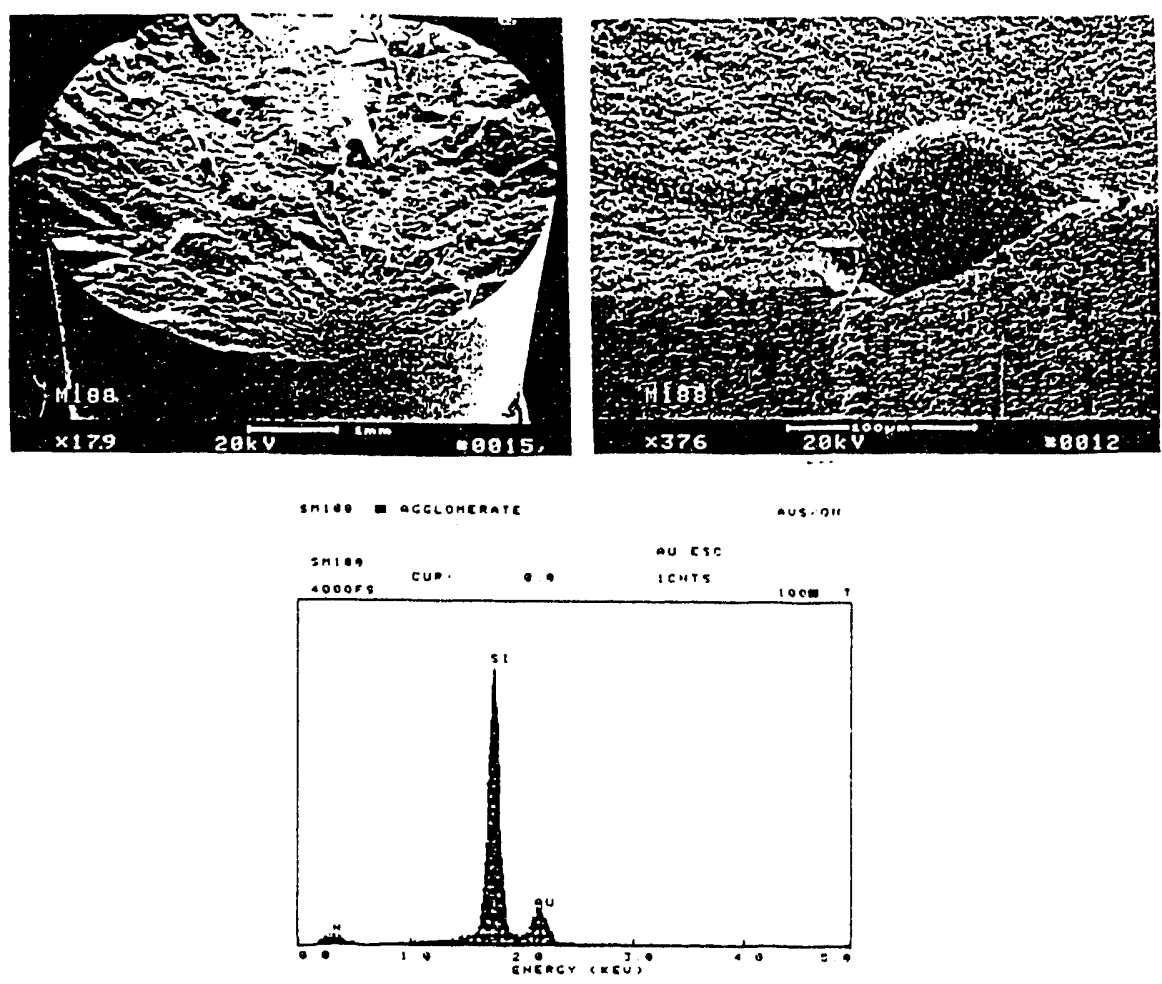

Figure 4-3: Failure origin Composed of an Agglomerate at the site of Machining Damage.

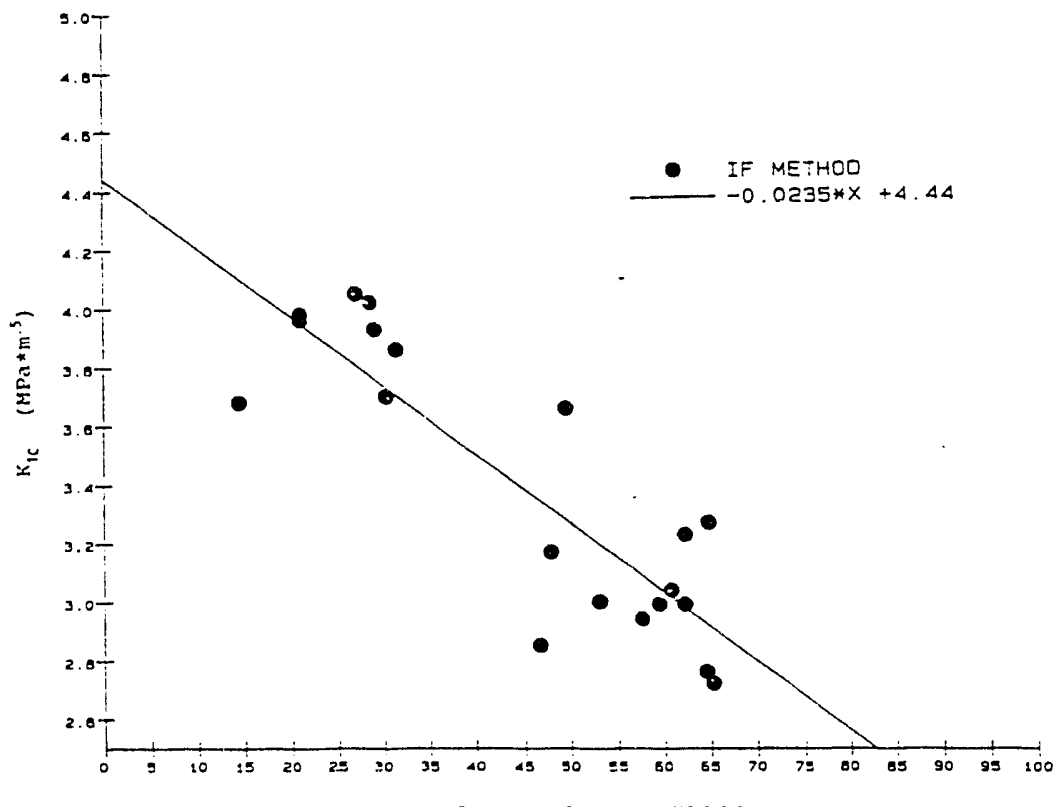

Figure 4-4: Fracture Toughress vs. \& Alpha Silicon Nitride 
Stress rupture tests were performed at $1230^{\circ} \mathrm{C}$ in air on three flexure specimens machined from tensile rods at an applied stress of 350 MPa. All three specimens survived in excess of 200 hours prior to termination of the testing.

\section{BASELINE PRESSURE CASTING VERIFICATION - ITERATION N}

A set of NSF tensile rods were cast using a repeat batch of baseline C-series slip to validate the baseline mechanical properties data. A total of 18 NSF tensile bars were cast for this third iteration (designated Iteration $N$ ) and tested for room temperature fast fracture strength. There was a high incidence of buttonhead failures (11 of 18) which fractography established to be due to machining damage at the buttonhead radius (BHR). These were machined prior to revision of the BHR surface finish specification which is discussed below. The 7 successful gage length fractures demonstrated exceptionally high strength levels ranging from 818-1056 MPa with a mean strength of 923 MPa and Weibull Modulus of 13; an improvement over the baseline C-series results above. On the basis of published data ${ }^{5}$ for commercially available structural Si3N4 materials (e.g. PY6, GN-10 and NT154) which indicated state-of-the-art RT average tensile strength at the $720 \mathrm{MPa}$ level, the data for this pressure cast material represented a significant technical advance.

Fractographic evaluation established that 6 of the 7 specimens failed from machining related defects. Three of these six failed from the effects of machining damage in combination with a surface pore or agglomerate, Figure 4-5. One specimen failed from a surface scratch normal to the longitudinal axis, Figure 4-6. This damage may have been inflicted during the rough grinding operation. There was one volume failure due to a $30 \mu \mathrm{m}$ agglomerate in the gage section.

Two elevated temperature $\left(1370^{\circ} \mathrm{C}\right)$ fast fracture tests were conducted in this iteration. In one case failure originated from one of the numerous $(2-20 \mu \mathrm{m}$ surface length) machining pullouts that marred the surface of this specimen, Figure 4-7. The second $1370^{\circ} \mathrm{C}$ test was conducted on a specimen without noticeable machining defects. Its failure origin was not evident from fractographic evaluation. The $1370^{\circ} \mathrm{C}$ strength levels were 395 and $397 \mathrm{MPa}$.

Two $1230^{\circ} \mathrm{C}$ stress rupture tests were conducted. The first test was loaded to an applied stress level of $250 \mathrm{MPa}$. It failed after 2 hours and the origin was traced to a surface machining groove and subsequent slow crack growth, Figure 4-8. The second test was run at a reduced stress of $200 \mathrm{MPa}$ but survived only 0.8 hours due to failure from a $165 \mu \mathrm{m} \mathrm{Fe/Y} \mathrm{rich} \mathrm{volume} \mathrm{inclusion,} \mathrm{Figure} \mathrm{4-9.}$ 


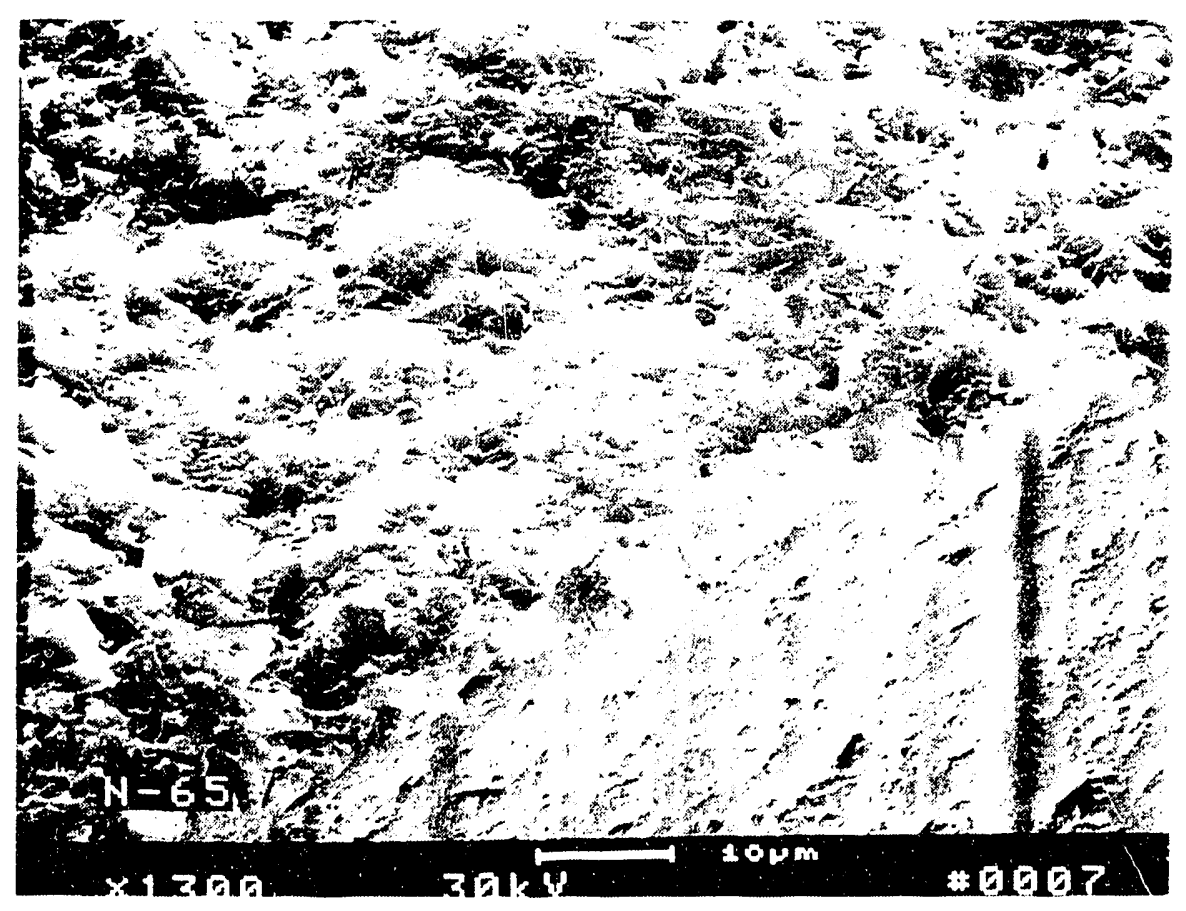

Figure 4-5: Failure Origin of Specimen ( $\sigma f=898 \mathrm{MPa}$ ) Showing Machining Marks and Agglomerate at the surface.

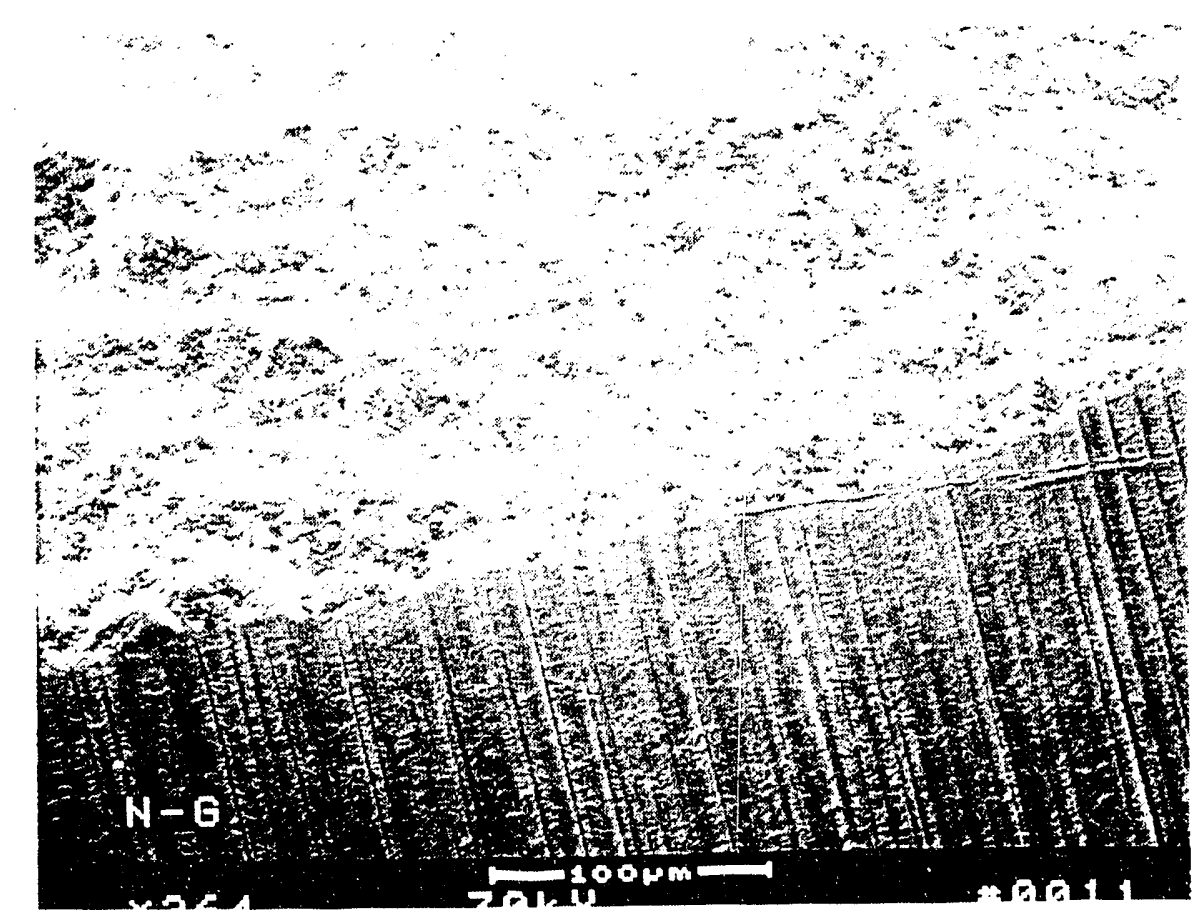

Figure 4-6: Failure origin of specimen (of=879 MPa) showing a severe circumferential scratch. 


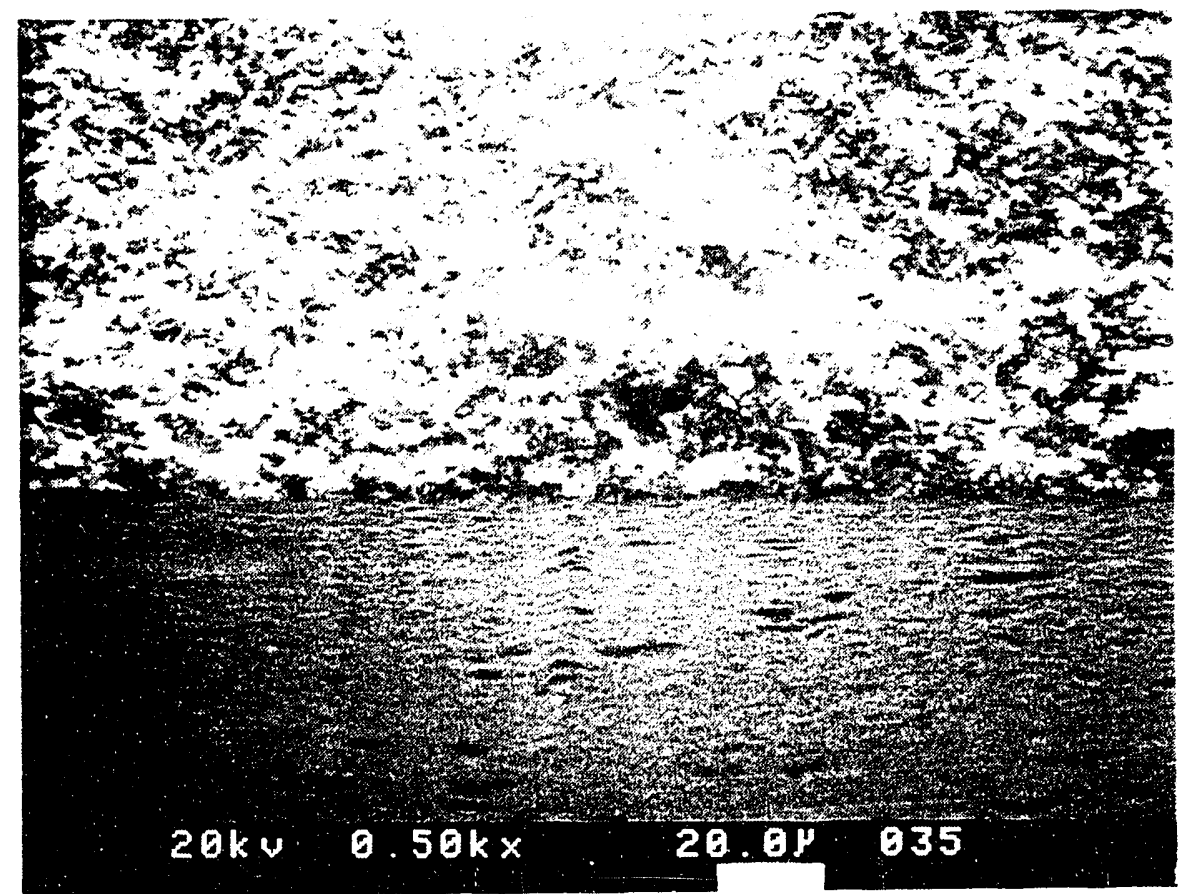

Figure 4-7: Machined surface and Failure origin of Specimen Fractured at $1370^{\circ} \mathrm{C}$ ( $\sigma f=395 \mathrm{MPa}$ ) Showing 2 - $20 \mu \mathrm{m}$ Machining Pullouts.

\section{MACHINING ISSUES}

The final cylindrical buttonhead tensile specimens were machined to the ORNL design with a gage diameter of $6.0+0.1 \mathrm{~mm}$. All specimens for the main body of strength data were machined using a specifically designed sop developed for this geometry. It should be noted that the gage section was longitudinally ground during both the roughing and finishing steps.

Two comprehensive studies were pursued to establish optimum machining practice. The first involved a machine shop qualification which established specifications for control of the buttonhead failure issue. The second involved specs for control of machining damage in the gage length as it influences tensile strength of NSF bars. 


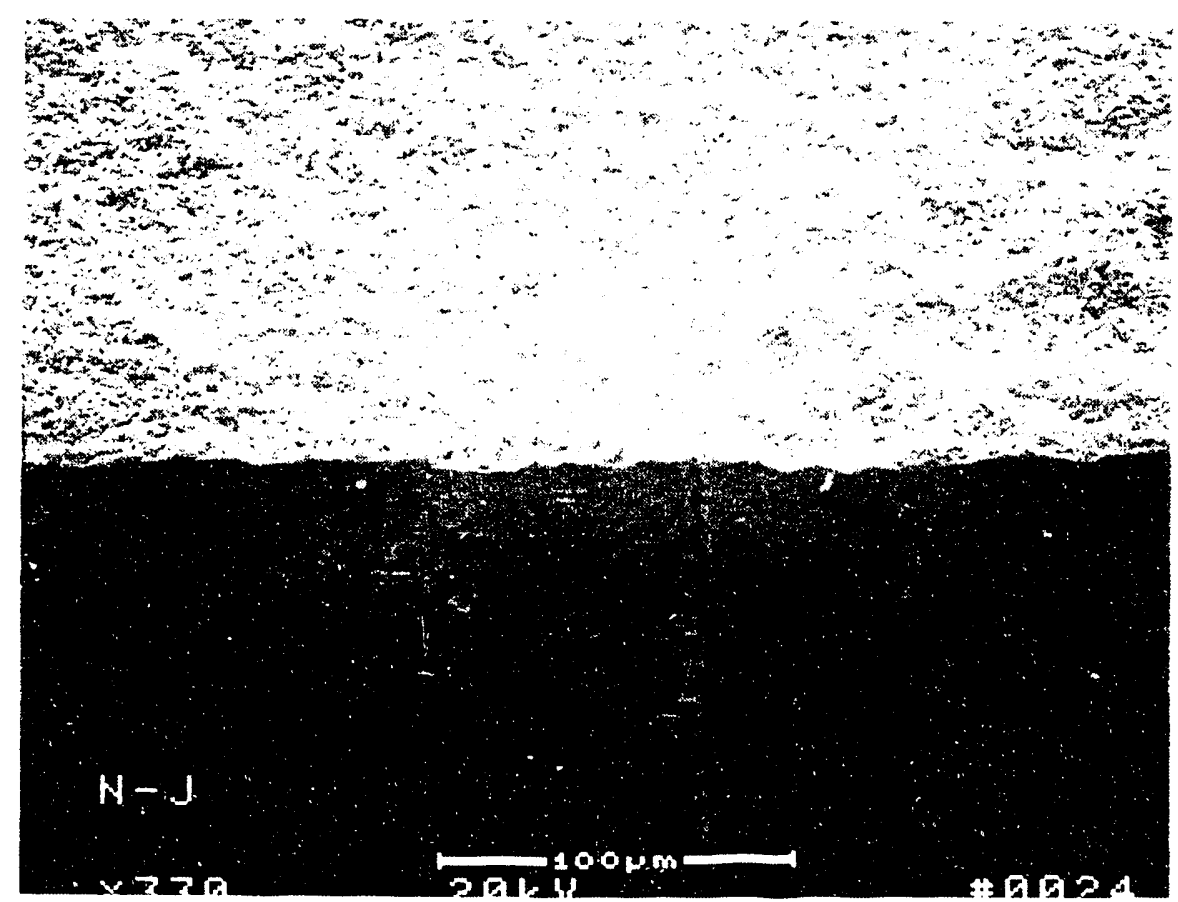

Figure 4-8: Failure origin of specimen Fraccured at $1230^{\circ} \mathrm{C}$ During stress Rupture showing a Machining Mark and slow crack Growth.

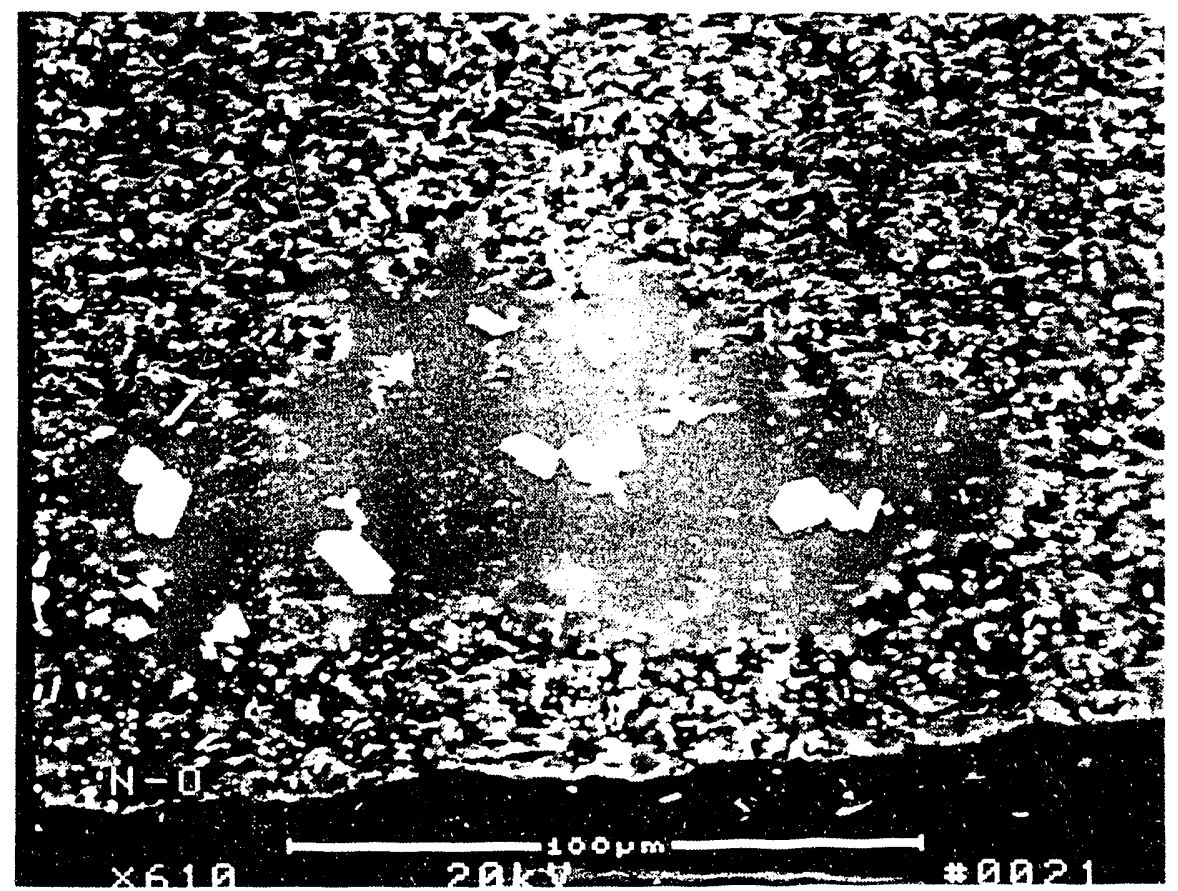

Figure 4-9: Failure origin of specimen Fractured at $1230^{\circ} \mathrm{C}$ During stress Rupture showing an Iron/Yttrium Rich Inclusion. 
Machining Related Buttonhead Failure

A significant number of buttonhead failures persisted for stage II specimens sven after resolution of the forming issues that caused critical flaws in the buttonhead radius region. Profilometry measurements were taken at the BHR of a large number of specimens of Iterations 3 and 4 (N and 0 ) to establish whether poor surface finish was the source of the problem. The results clearly show that surface finish was the major cause of the problem.

An experiment was devised to establish how machining practice influences tensile bar strength. Three machine shops were chosen to participate in the study with each employing its proprietary procedure (type diamond wheel, fixturing, method of BHR grinding, etc.) to meet a surface finish specification $\left(R_{a}\right)$ at the gage and $B H R$ of $0.41 \mu \mathrm{m}$. The three shops involved in the study routinely met and exceeded this requirement in the gage length, with $R_{\text {a }}$ values in the .13-.18 $\mu$ in range. The finish of the BHR varied significantly both above and below the specification and this provided the basis for correlating buttonhead failure to finish.

The overall average BHR surface finish of NSF specimens which failed at the BHR was found to be $0.51 \mu \mathrm{m}$. The average for non-BHR failure was $0.38 \mu \mathrm{m}$. Table 4-6 gives the average $R_{a}$ measured at the $B H R$ of specimens machined at the three shops, designated as shops $A, B$ and C. Also listed are the percentages of specimens from each shop which failed at the BHR. Shop A finished to an average $R_{a}$ of $0.49 \mu \mathrm{m}$ with a resulting 518 BHR failures. Shop $C$ supplied bars with an average $R_{a}$ equal to $0.25 \mu \mathrm{m}$ and this resulted in only 48 BHR failures ( 1 of 24 ). The $R_{\text {a }}$ results from shop $B$ averaged $0.33 \mu \mathrm{m}$, within specification, but nonetheless 338 of the specimens failed at the BHR. The data clearly show that a more stringent surface finish specification was required in the buttonhead region for the high strength materials being processed in this program.

Table 4-6: Buttonhead Radius Surface Finish - Failure Data

\begin{tabular}{|c|c|c|c|}
\hline $\begin{array}{c}\text { Machine } \\
\text { Shop }\end{array}$ & $\begin{array}{c}\text { Average } \mathrm{R}_{\mathrm{a}} \\
\mu \mathrm{m}\end{array}$ & $\begin{array}{c}\text { Buttonhead } \\
\text { Failures } \\
8\end{array}$ & $\mathrm{n}$ \\
\hline \hline A & $.49+/-.06$ & 51 & 38 \\
\hline B & $.33+/-.08$ & 33 & 18 \\
\hline C & $.25+/-.06$ & 4 & 24 \\
\hline
\end{tabular}

As a result of this study, the Ra spec was reduced from $0.41 \mu \mathrm{m}$ (16 $\mu \mathrm{in})$ to $0.25 \mu \mathrm{m}(10 \mu \mathrm{in})$ at the buttonhead radius and to $0.2 \mu \mathrm{m}$ (8 $\mu i n)$ along the gage length.

The machining requalification study described above was conducted in part with specimens from Iteration 0 . Nineteen $(19)$ of 32 room 
temperature NSF fast fracture tests were successful gage section failures and resulting strength values ranged from 586 to $950 \mathrm{MPa}$ and averaged $789 \mathrm{MPa}$, with a characteristic strength of $839 \mathrm{MPa}$ and Weibull Modulus of 8.5. In spite of excellent surface finish $\left(R_{a}=.13-.18 \mu \mathrm{m}\right)$, SEM fractography suggested that the majority of these gage failures initiated from machining defects, ranging from $5 \mu \mathrm{m}$ wide axial grinding marks to $>750 \mu \mathrm{m}$ transverse scratches, Figure 4-10 a,b,c. Associated with the axial grind marks are subsurface thumbnail shaped cracks. Fracture mechanics was employed to provide critical flaw size predictions as a guide to interpretation of the SEM fractography as is discussed below. A small percentage of the failure origins involved inclusions ranging in size from 20 to $135 \mu \mathrm{m}$, Figure 4-11. The surface finish comparisons cosducted in the machining study indicated no statistically significant surface finish difference in the gage length region of specimens machined by shops $A, B$ and $C$. Nonetheless, the strength data for a subset of 8 bars which was machined by shop $C$ was so outstanding ( $\sigma 0=904 \mathrm{MPa}, \mathrm{m}=28$ ) that the machining method employed by shop $c$ (roughing as well as finish grinding) was incorporated as sOP in further iterations.

\section{Fractography - strength Correlation}

A limited amount of fractography data was analyzed to establish quantitative relationship between strength and flaw size. Strength data for specimens which failed from machining darnage related surface flaw failures are plotted as a function of $1 / \sqrt{ }$ a in Figure 4-12. The maximum depth of the surface flaw (not the surface length) is plotted as the dimension a. There is excellent agreement between the regression line and the semi-circular surface crack fracture mechanics prediction ${ }^{6}$ :

$$
\sigma_{\varepsilon}=0.71 \quad K_{1 c} / \sqrt{ } a
$$

This prediction was developed using the iteration average $\mathrm{kic}$ value of $5.35 \mathrm{MPa} / \mathrm{m}$. This correlation supports the view that strength impairing machining defects take the form of distinct surface flaws beneath individual grinding grooves. The prediction following from the long surface crack model' $(0.71$ replaced by 0.50 in equation (4-1)) can be seen to fall significantly below the test data.

\section{Gage Length Machining Optimization}

Four distinct machining procedures (including the original SOP) were evaluated for their influence on tensile strength as an additional experiment. The four machining procedures differed according to intermediate grinding steps, diamond wheel grit size and depths of cut as outlined in Table 4-7. This experiment maintained the $R_{\text {. }}$ specs while altering the rough and intermediate grinding steps with the goal of reducing subsurface damage due to the prior step. On the basis of the grinding parameters employed, the machining procedures were ranked according to operational precision from \#1 to \#4, with \#4 being the most precise procedure. Consistent with the focus on machining damage 
effects, the specimens were not given post-machining heat treatments for the purpose of this study. A total of 72 specimens from 3 HIP runs were machined and tested for this study. The procedures were rated by statistical analyses of tensile strength data.

To properly evaluate the differences between the four procedures, only those specimens that failed from surface originating flaws were considered (64 of 72). An ANOVA test was performed to compare differences in the data. Of the four machining procedures, the procedure \#1 (original SOP) had the least variability, Table 4-8. In spite of the major differences in operational precision, there was no statistically significant effect of procedure on strength as shown in Table 4-8. $R_{y}$ (maximum peak to valley) surface finish values were found to differ for \#1 \& $\# 2$ vs. \#3\& \#4 and are also shown in Table 4-8. since the primary consideration was assuring a high reliability, the procedure with the least variability (\#1) was chosen for future machining and can be used as a benchmark for further machining experiments.
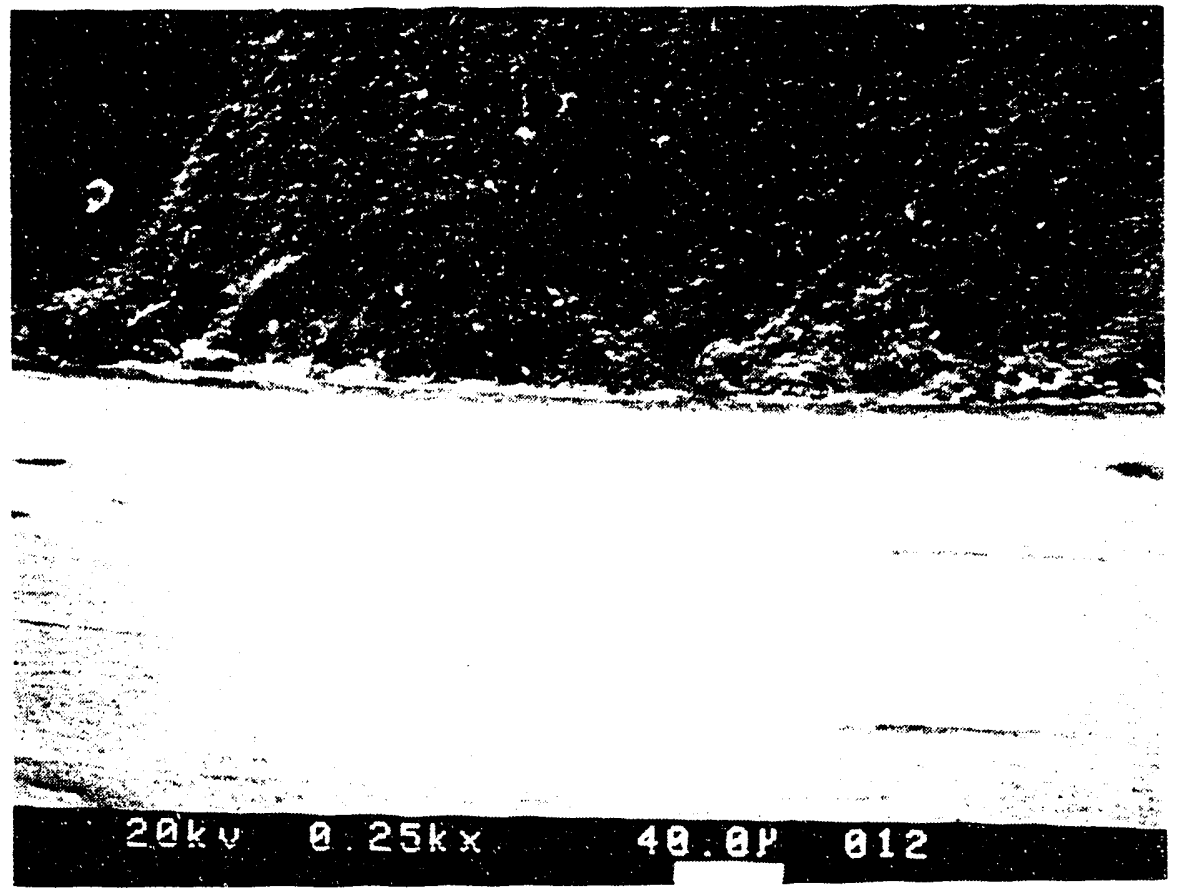

Figure 4-10a: Failure origin ( $\sigma f=596 \mathrm{MPa}$ ) Showing a Severe Radial Grinding Mark. 


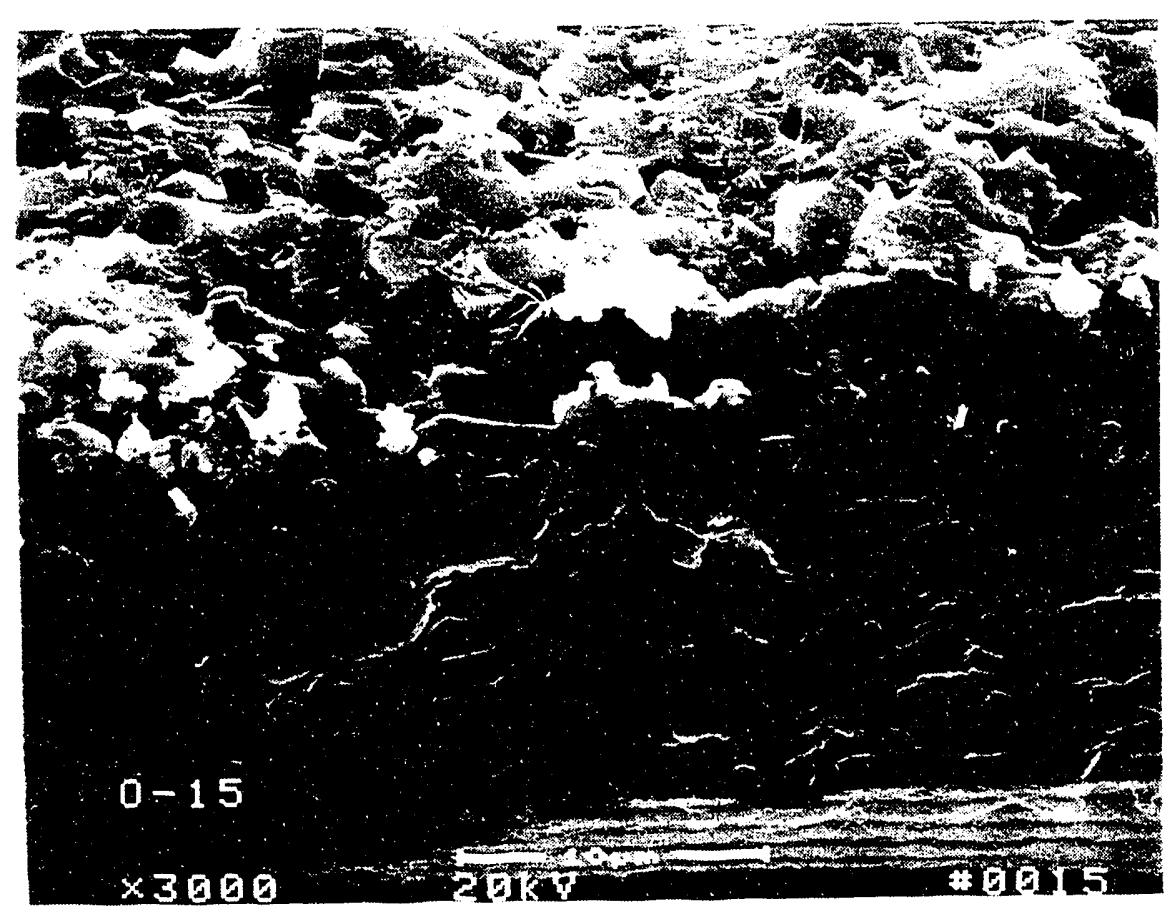

Figure 4-10b: Failure origin ( $\sigma f=899 \mathrm{MPa}$ ) Showing a $5 \mu \mathrm{m}$ wide Machining Mark.

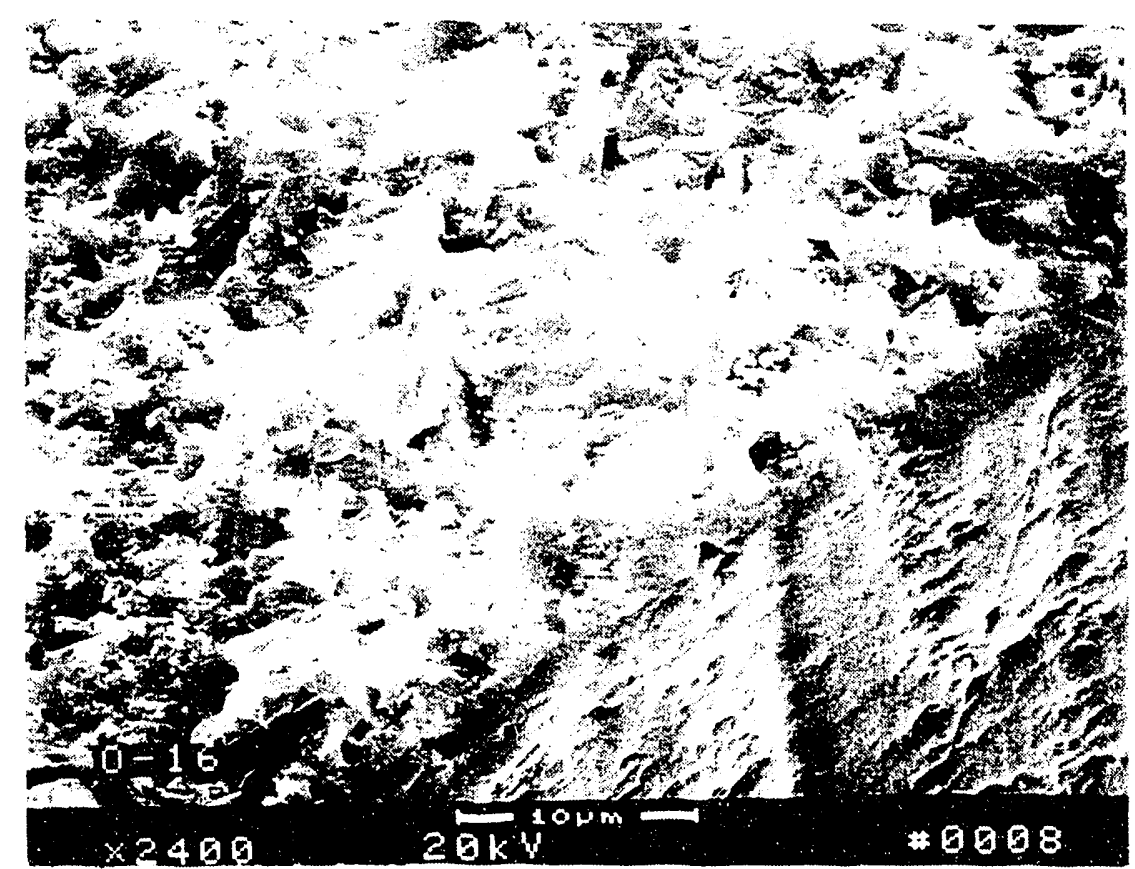

Eigure $4-100$ :

Eailure origin (of=8s9 MPa) Showing a 5 um wide Machining Groove and Sub-surface Thumbail shaped Elaw 


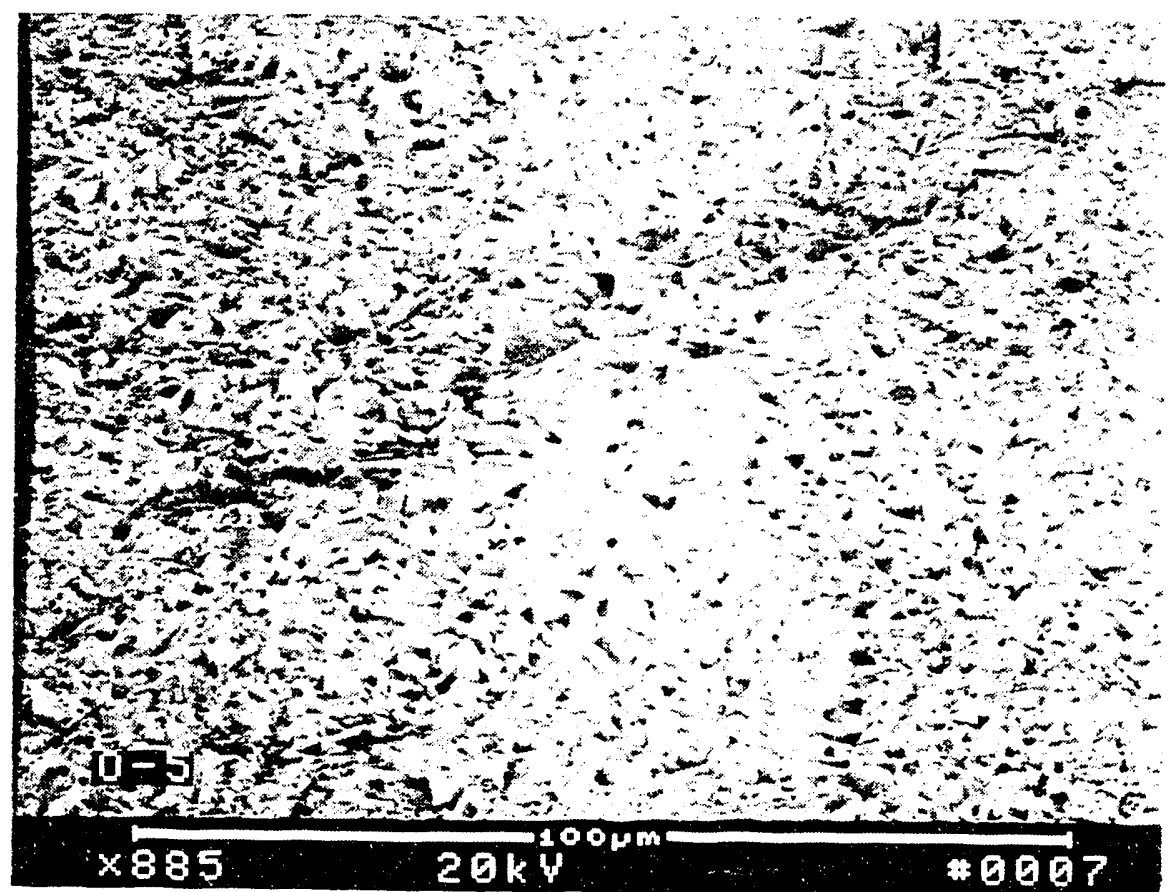

Figure 4-11: Failure origin ( $\sigma f=787 \mathrm{MPa}$ ) showing an Inclusion Containing $\mathrm{Fe}, \mathrm{Cr}, \mathrm{Ni}$.

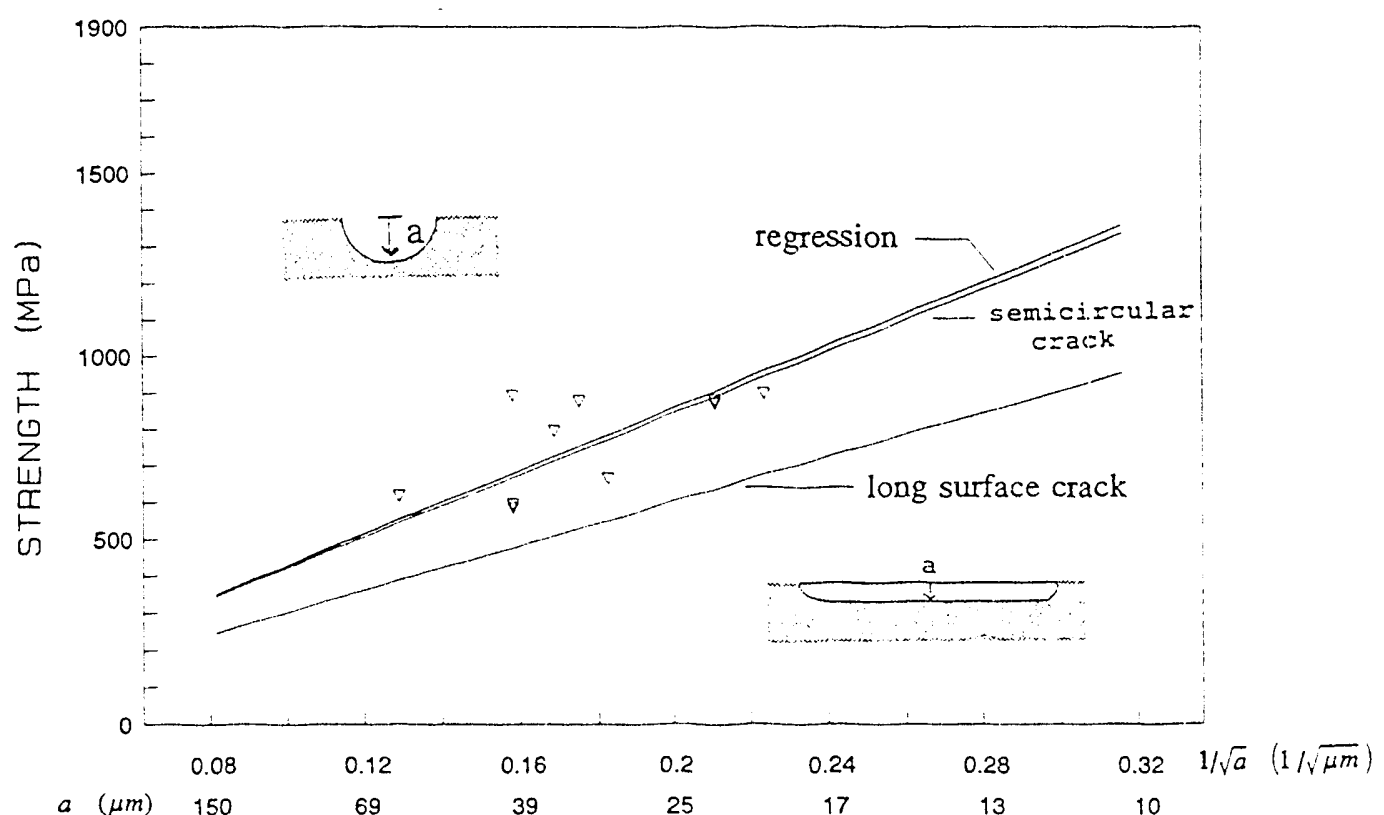

Machining Damage Failure Origins

Pressure Casting - Iteration $\mathrm{O}$

Figure 4-12: Strength-flaw size Correlation for Machining Damage Failure origins. 
Table 4-7: Procedure steps for Machining Tensile Rods.

\begin{tabular}{||l|l|l|l|}
\hline Procedure & $\begin{array}{c}\text { Roughing } \\
\text { step }\end{array}$ & \multicolumn{1}{|c|}{$\begin{array}{c}\text { Intermediate } \\
\text { step(s) }\end{array}$} & $\begin{array}{c}\text { Finishing } \\
\text { step }\end{array}$ \\
\hline \#1 Original sop & 180 grit & - & $\begin{array}{l}320 \text { grit } \\
(0.51 \mathrm{~mm})^{*}\end{array}$ \\
\hline \#2 Experimental & 320 grit & 320 grit & 320 grit \\
\hline \#3 Experimental & 180 grit & 320 grit & $\begin{array}{l}800 \text { grit } \\
(0.15 \mathrm{~mm})\end{array}$ \\
\hline \#4 Experimental & 180 grit & $\begin{array}{l}320,400,600 \text { grit } \\
(0.05 \mathrm{~mm})(0.05 \mathrm{~mm})\end{array}$ & $\begin{array}{l}800 \text { grit } \\
(0.05 \mathrm{~mm})\end{array}$ \\
\hline
\end{tabular}

* $(0.51 \mathrm{~mm})$ refers to amount of stock removed by that step.

Table 4-8: Machining Procedure strength Data.

\begin{tabular}{|c|c|c|c|c|}
\hline Procedure & $\begin{array}{c}\text { Mean } \\
\text { Strength } \\
\text { (MPa) }\end{array}$ & $\begin{array}{c}\text { Standard* } \\
\text { Error } \\
\text { (MPa) }\end{array}$ & $\begin{array}{c}\text { Surface } \\
\text { Finish R } \\
\text { (microns) }\end{array}$ & $\begin{array}{c}\text { Number of } \\
\text { Specimens } \\
\mathrm{n}\end{array}$ \\
\hline 1 (SOP) & 812 & 18 & 1.26 & 31 \\
\hline 2 (Experimental) & 767 & 35 & 1.33 & 10 \\
\hline 3 (Experimental) & 847 & 30 & 0.77 & 14 \\
\hline 4 (Experimental) & 845 & 42 & 0.68 & 9 \\
\hline
\end{tabular}

* standard error $=$ standard deviation $/ \sqrt{ } \mathbf{n}$

\section{TENSILE DATA FOR PROCESS OPTIMIZATION}

The stage II experimental plan involved 16 processed batches of silicon nitride and each of the experimental blocks was evaluated through room temperature tensile tests using approximately thirty tensile rods amounting to a total of over 500 rods tested. These data are summarized in Figure 4-13. The doublets for Iteration $V$ and $z$ are from the oxidation treatment experiments while for Iteration 0 the 3 values correspond to the 3 machining procedures employed. 


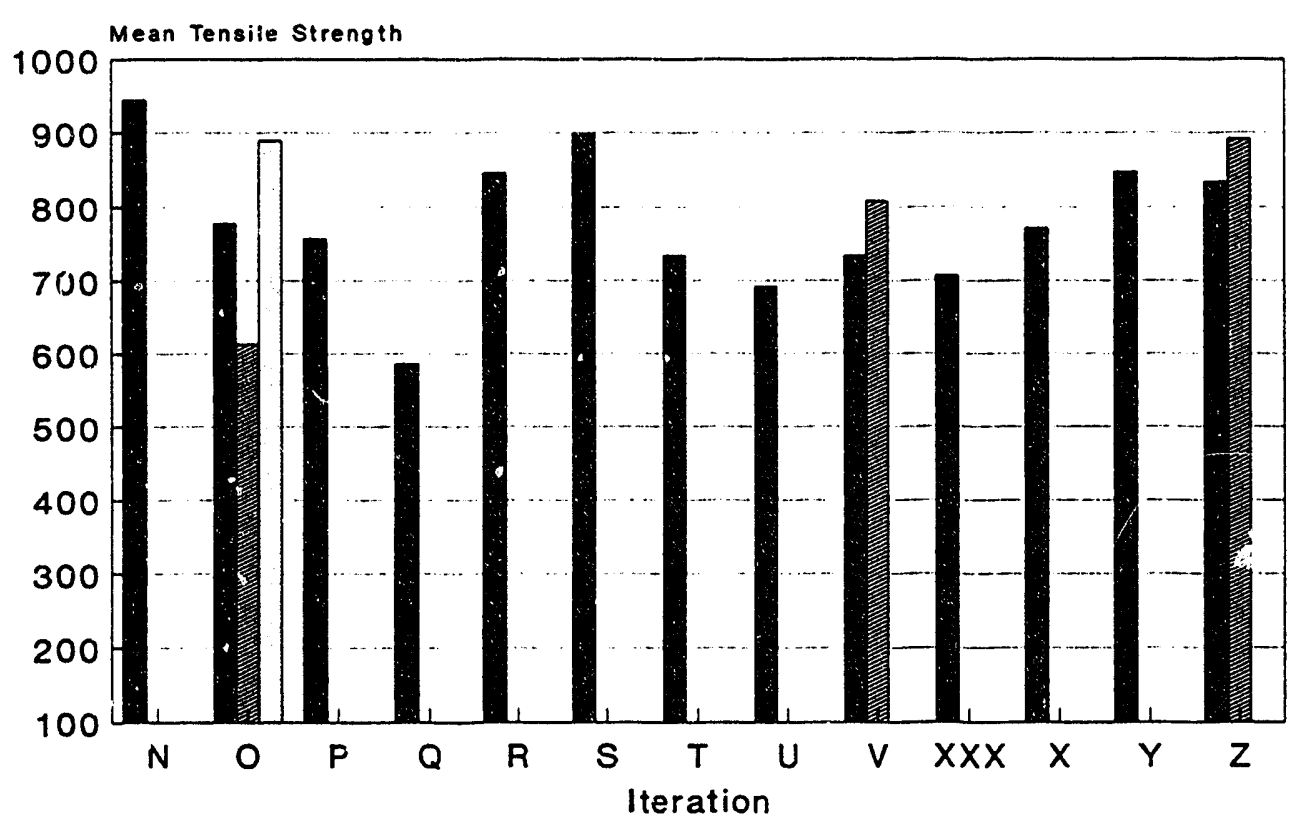

Figure 4-13: Mean Tensile strengths From stage II Iterations

The unit operation control variables evaluated in the experimental plan were:

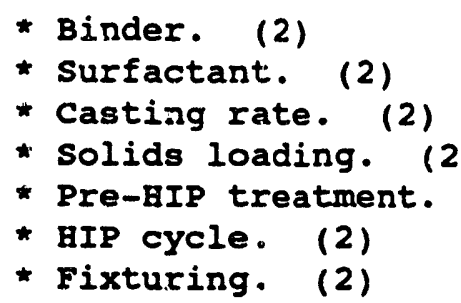

The results of the experimental plan defined the set of optimum process conditions used in the stage III process demonstration.

\section{Critical Flaw Population Shifts}

By the nature of the experimental plan, there were significant differences from iteration to iteration in the tensile strength levels corresponding to the response surface, Figure 4-13. Iterations $\mathrm{xxx}, \mathrm{x}$, $Y$ and $z$ were conducted at a time when wear of processing equipment was recognized (NDE, fractography) as a significant source of contamination. The compilation of strength and fractography data for these iterations provide a valuable database on shifts in critical flaw populations. The shifts resulted from corrective actions which el minated wear related iron contamination midway through the period.

Table 4-9 gives the strength statistics for the four sets of tensile rods which totaled 135 samples. As each subsequent batch was "cleaned up" by elimination of iron contaminating sources the mean strength jusceased by over $170 \mathrm{irfa}$. Optical ana seir fractography were performed on all 135 specimens and the results are summarized in Table 
4-10. There was a significant shift from volume failures to surface failures with the elimination of iron contamination from Iteration XXX up to Iteration $\mathrm{z}$. The surface flaw type "Am", amorphous region, was found to be related to the post-machining heat treatment. The other significant flaw type "Mp", zone of microporosity, occurs in the volume and may be related to the densification process. It is apparent from Iteration $z$ data that surface flaws related to machining are the major strength and reliability limiting defects.

Table 4-9: RT Tensile Strength Data for Batches Xxx - 2

\begin{tabular}{|c|c|c|c|c|c|}
\hline Batch & $\begin{array}{c}\text { Mean } \\
\text { Strength } \\
\text { (MPa) }\end{array}$ & $\begin{array}{c}\text { Std. } \\
\text { Dev. } \\
\text { (MPa) }\end{array}$ & $\begin{array}{c}\text { Characteristic } \\
\text { Strength } \\
\text { (MPa) }\end{array}$ & $\begin{array}{c}\text { Weibull } \\
\text { Modulus } \\
\mathrm{m}\end{array}$ & $\begin{array}{c}\text { No. of } \\
\text { Specimens }\end{array}$ \\
\hline $\mathrm{XXX}$ & 707 & 88 & 746 & 9.0 & 25 \\
\hline $\mathrm{X}$ & 771 & 105 & 816 & 8.5 & 18 \\
\hline $\mathrm{Y}$ & 847 & 103 & 892 & 9.4 & 45 \\
\hline $\mathrm{Z}$ & 878 & 108 & 924 & 9.4 & 47 \\
\hline
\end{tabular}

Table 4-10: Fractography summary for Batches Xxx - 2

\begin{tabular}{|c|c|c|c|c|c|c|c|c|}
\hline \multirow[t]{2}{*}{ Batch } & \multicolumn{2}{|c|}{ Location } & \multicolumn{5}{|c|}{ Flaw Type (8) } & \multirow{2}{*}{$\begin{array}{c}\text { Size } \\
\text { Range } \\
(\mu \mathrm{m})\end{array}$} \\
\hline & $8 \mathrm{~V}$ & 85 & I & Mp & $\mathbf{M}$ & Am & $\mathbf{u}$ & \\
\hline $\mathrm{xxx}$ & 68 & 32 & 72 & 0 & 20 & 0 & 8 & $>100$ \\
\hline $\mathrm{x}$ & 29 & 71 & 41 & 6 & 24 & 12 & 17 & $15-50$ \\
\hline $\mathbf{X}$ & 2 & 98 & 0 & 2 & 28 & 68 & 2 & $10-80$ \\
\hline $\mathbf{z}$ & 29 & 71 & 0 & 40 & 45 & 9 & 6 & $5-65$ \\
\hline
\end{tabular}

$$
\begin{aligned}
& v=\text { volume } \\
& S=\text { surface }
\end{aligned}
$$

$$
\begin{aligned}
& I=\text { iron inclusions } \\
& M p=\text { microporosity } \\
& M=\text { machining damage } \\
& A m=\text { amorphous region } \\
& U=\text { unknown }
\end{aligned}
$$

Optimized Process Tensile Data

Near the conclusion of Stage II, a total of 53 tensile specimens were fabricated by the "optimized process". This set was found to have a mean strength of $888 \mathrm{MPa}$ and a range from 548 to $1091 \mathrm{MPa}$. The corresponding tensile strength distribution is plotted in Figure 4-14. Competing risk Weibull distributions were generated for surface and volume flaw types and are shown in Figure 4-15. The Weibull moduli are 
9.3 and 9.9 for surface and volume flaws, respectively. It is interesting to note that at any given applied stress level the probability of failure is higher for a surface flaw than for a volume fla'w according to this data.

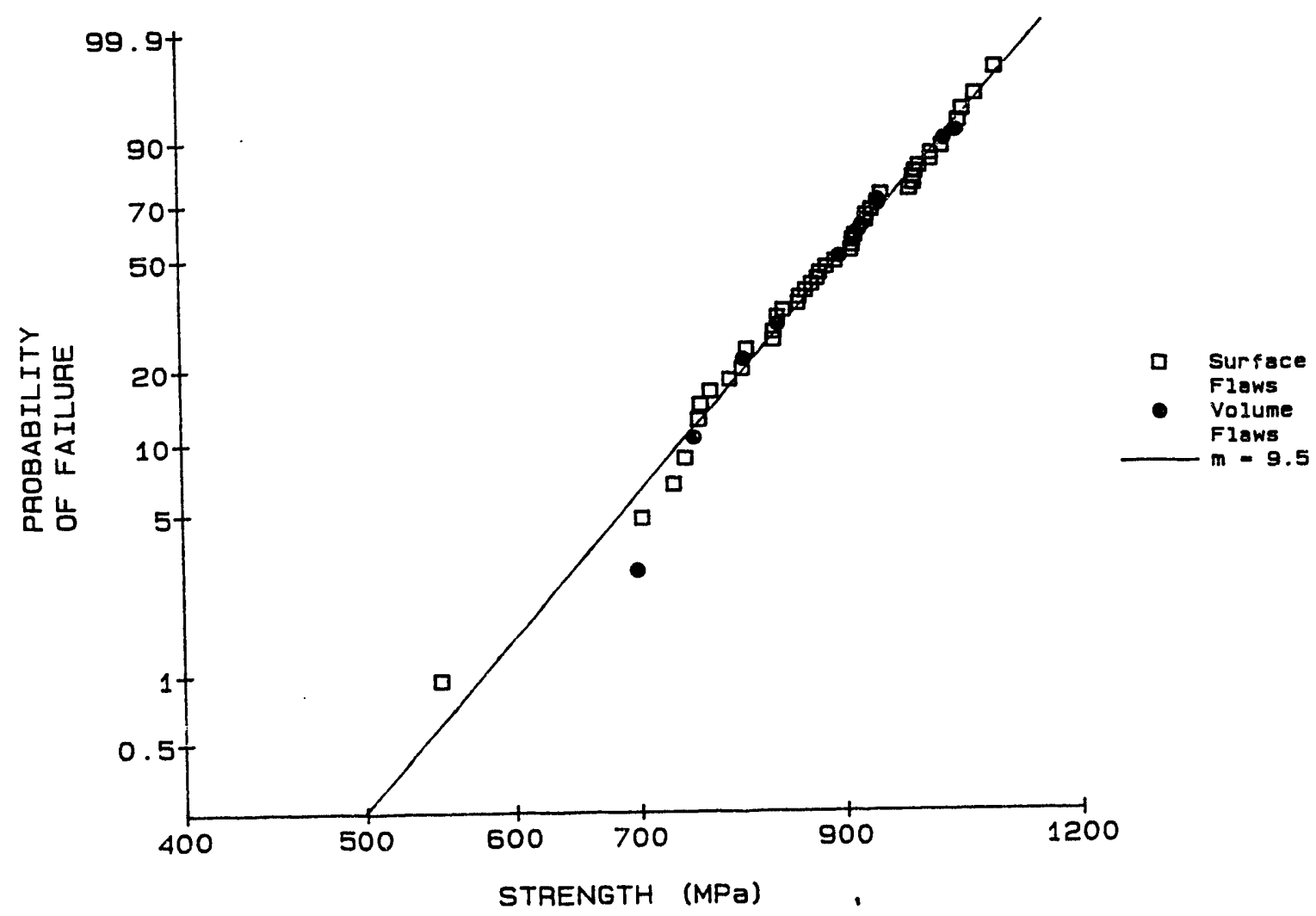

Figure 4-14: Weibull Plot Assuming an Unimodal Strength Distribution.

The surface flaw strength distribution in Figure 4-15 visually suggests 3 regimes within the distribution. Detailed fractography observed that within the surface flaw population there were indeed 3 flaw origin types: surface amorphous regions and two types of machining damage. The volume flaws are of one type and are characterized as agglomerate-porous zones. Figures $4-16 a, b, c, d$ show micrographs of these flaw types. Included are atypical machining damage, normal machining damage, si-rich amorphous regions and zones of microporosity.

Based on the fractographic data, another competing risk Weibull analysis was performed, distinguishing the 3 surface flaw and the 1 volume flaw populations. For clarity, only the three surface flaw distributions are shown in Figure 4-17. Since the strength distribution data (mean $=889 \mathrm{MPa}$ and $m=9.9$ ) from the volume failures are from only one flaw type, the modulus does not change. However, the three distributions from the surface flaw data show very different results. 


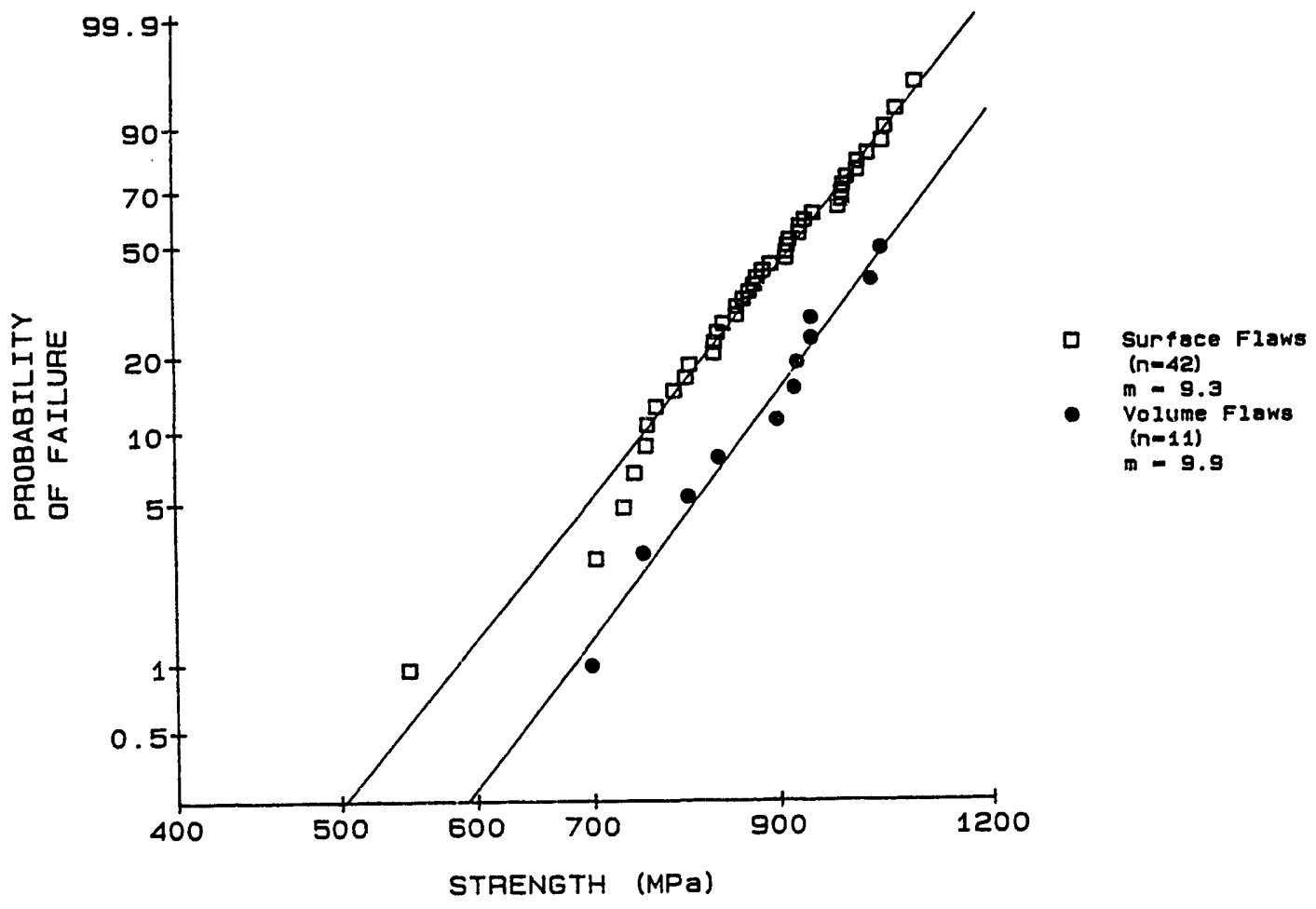

Figure 4-15: Weibull Plot Using Competing Risk for surface and volume Failure

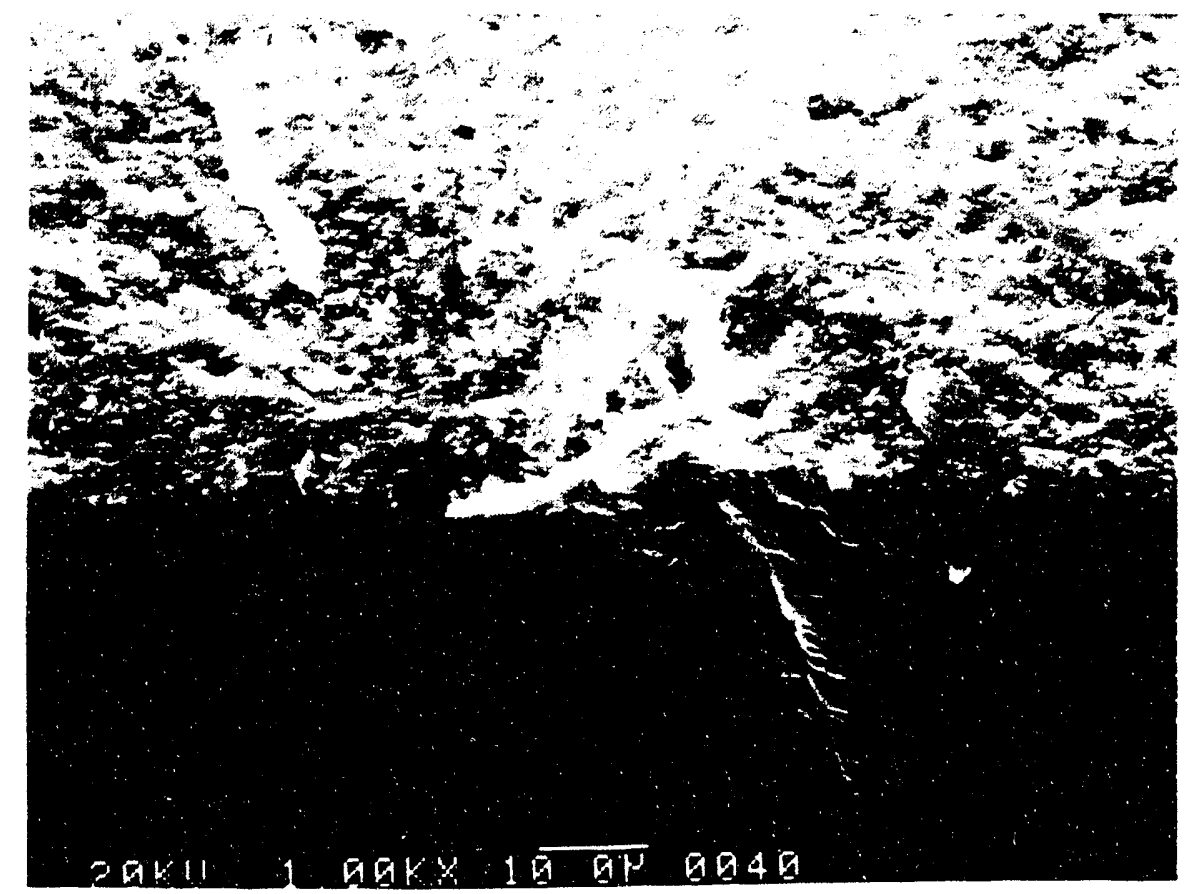

Figure 4-16a: Failure origin ( $\sigma \mathrm{f}=685 \mathrm{MPa}$ ) Showing Atypical Machining Damage 


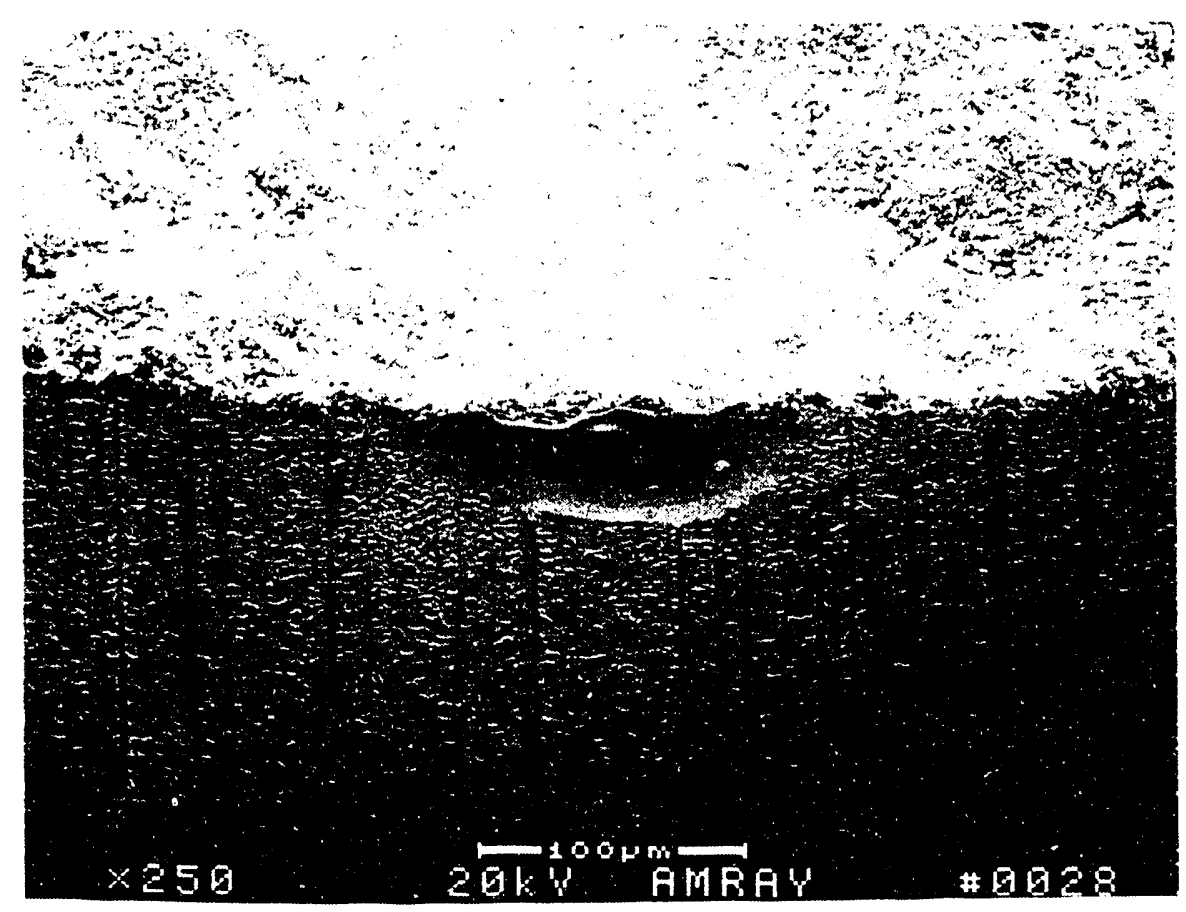

Figure 4-16b: Failure origin ( $\sigma f=921 \mathrm{MPa}$ ) Showing an Amorphous Region

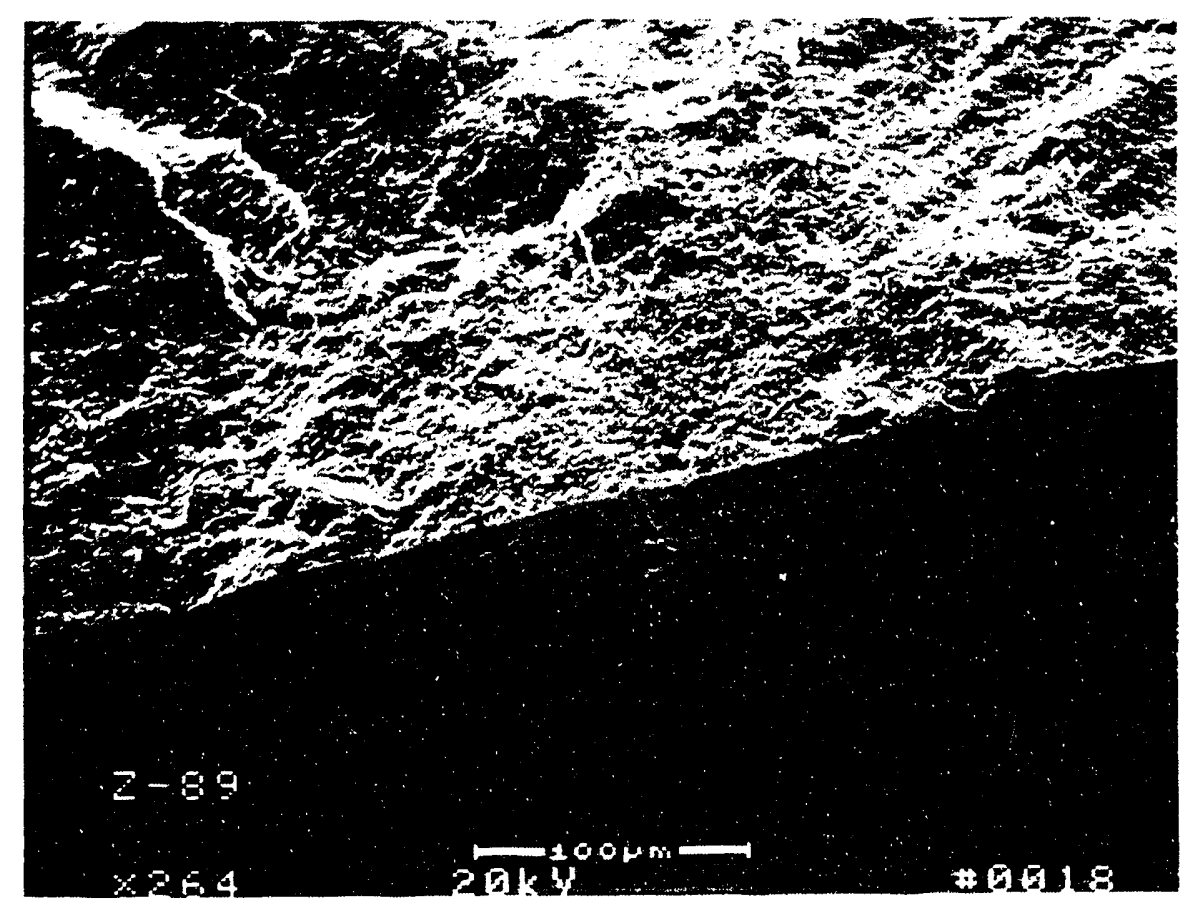

Figure 4-16c: Failure origin (of $=944 \mathrm{MPa}$ ) showing Normal Machining Damage 


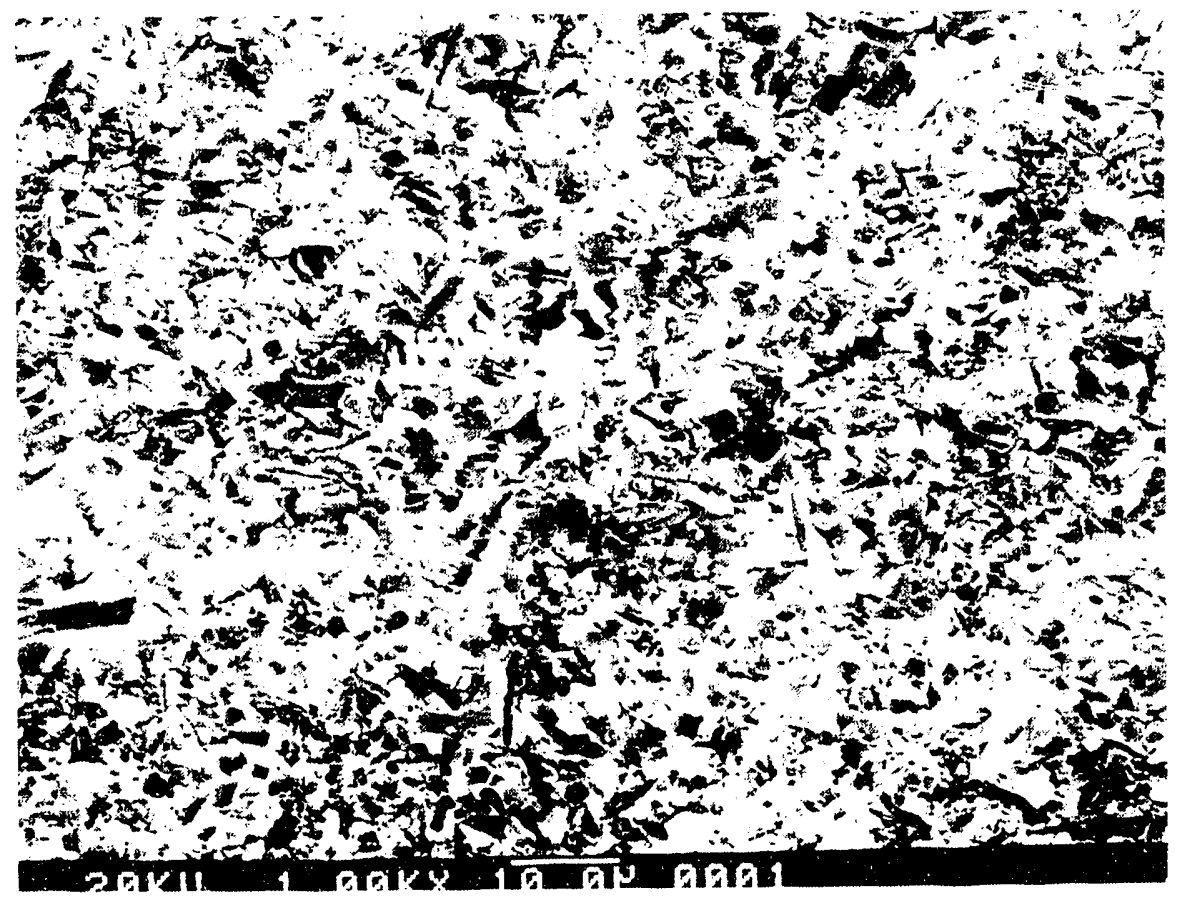

Figure 4-16d:

Failure origin ( $\sigma f=1040 \mathrm{MPa}$ ) showing a Volume Defect Containing Microvoids.

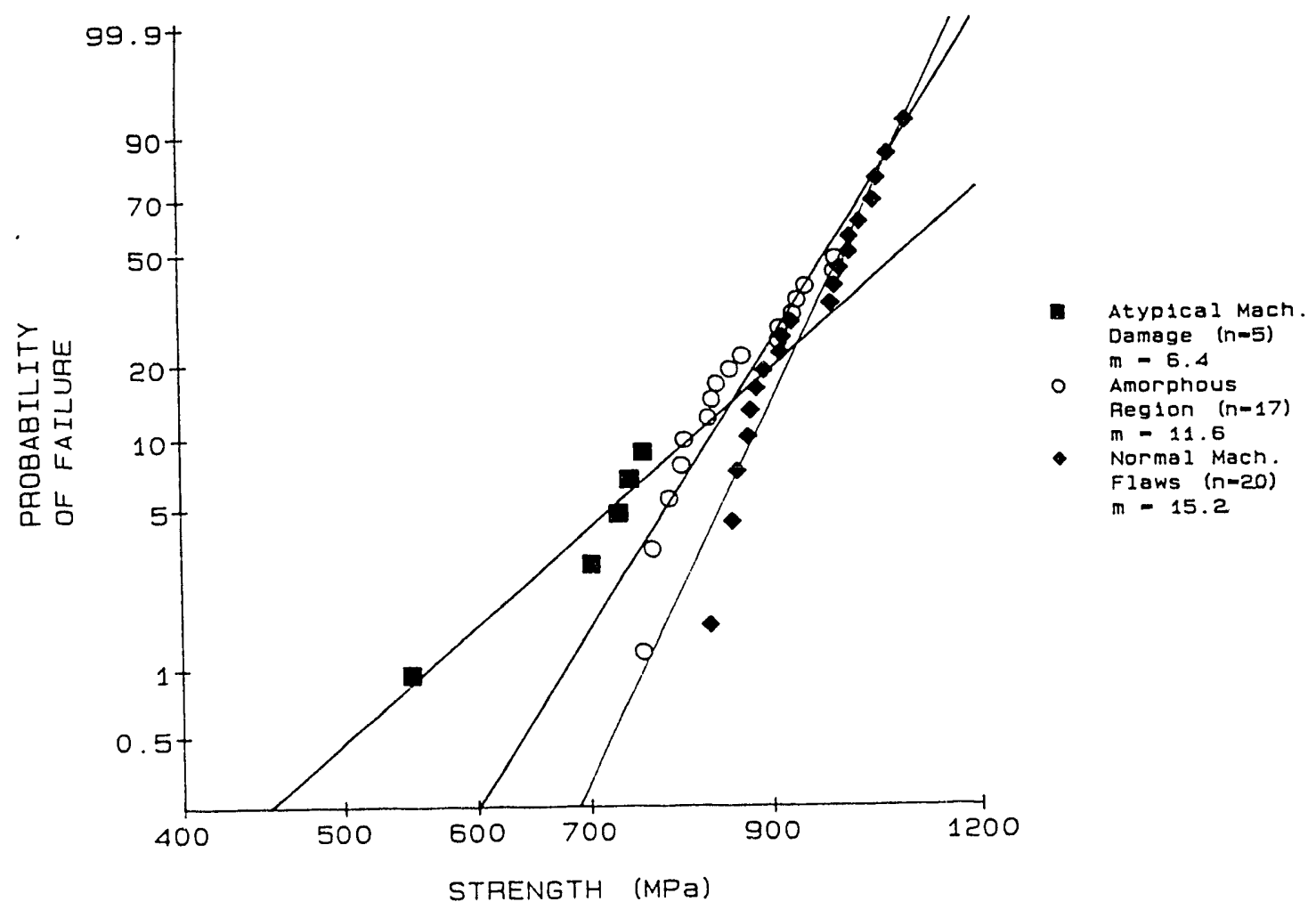

Figure 4-17: Competing Risk Weibull plot for Three surface Flaw Types. 
As expected, the lowest strength and Weibull Modulus (mean $=696$ MPa and $m=6.4$ ) were from specimens having atypical machining damage as the critical flaw origin. The middle strength values (mean $=867 \mathrm{MPa}$ and $m=11.6$ ) are from si-rich amorphous origins. These defects developed during the annealing process and their precursors may be from local inhomogeneities in the microstructure or from subsurface cracks formed during machining. These strengths overlap both the atypical machining damage and normal machining damage. The third and highest mean strength and Weibull Modulus (954 MPa and 15.2) are from specimens that had surface flaws from normal machining (procedure \#1).

\section{EFFECTS OF HEAT TREATMENT ON TENSILE STRENGTH}

Iteration $v$ specimens were used to evaluate the effects of postBIP and post-machining heat treatments on room and elevated temperature tensile strength. The post-HIP treatment is conducted prior to machining to crystallize the secondary phases present after densification. The post-machining treatment is performed to anneal and oxidize (blunt) surface and near surface flaws caused by the grinding process. A total of 63 room temperature and 13 elevated temperature $\left(1370^{\circ} \mathrm{C}\right)$ tensile tests were conducted on specimens which comprised the $2 \times 2$ experimental matrix.

The mean values of tensile strength for the four categories of room temperature tests are plotted in Figure 4-18. It is apparent that in general, crystallization (XTAI) can decrease the RT strength although the data suggest the effect is statistically insignificant, while oxidation (OXID) increases it. ANOVA data analysis suggests a 18 XTAL mean strength decrease and an 118 oxID increase. The individual mean strength values were $743,719,806$ and $811 \mathrm{MPa}$ for the four conditions (NONE, XTAL, OXID, BOTH) plotted in Figure 4-18.

While the oxidation treatment appears to have a significant effect on the character of the surface flaw population, apparently crystallization does not influence the room temperature strength limiting volume defects. There is a small interaction of the two treatments which is also displayed in Figure 4-18.

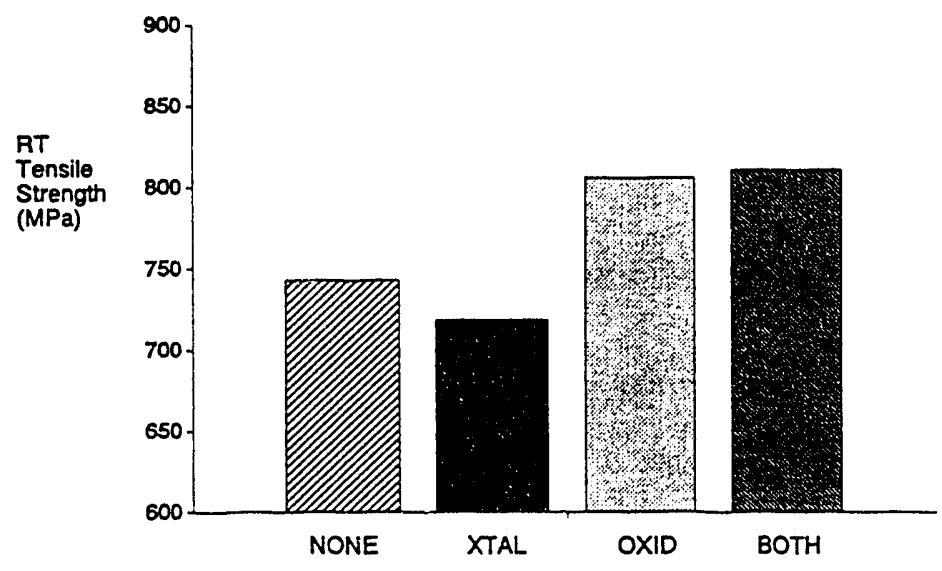

Figure 4-18:

Mean Room Temperature Tensile Strength as a Function of Heat Treatment Condition. 
Iteration $\mathrm{z}$ specimens were subsequently used to validate the oxidation strength enhancement observed in Iteration v. Fifteen (15) specimens were tested in the as-machined condition. These had a mean RT strength of $834 \mathrm{MPa}$. Thirty-two (32) specimens were given a postmachining heat treatment and were tested for RT fast fracture strength. The resultant mean tensile strength for these 32 specimens was $891 \mathrm{MPa}$. This represented a 78 strength enhancement by the oxidation treatment which is consistent with the Iteration $v$ experimental results.

The $1370^{\circ} \mathrm{C}$ mean tensile strength data are summarized in Figure 419. Without either treatment (NONE), the mean was $347 \mathrm{MPa}$ while with both, the mean was $480 \mathrm{MPa}$, a significant increase of 388 . The interaction effect is evident in Figure 4-19. ANOVA suggests that an 188 increase is attributable to crystallization and a 168 increase is attributable to oxidation alone.

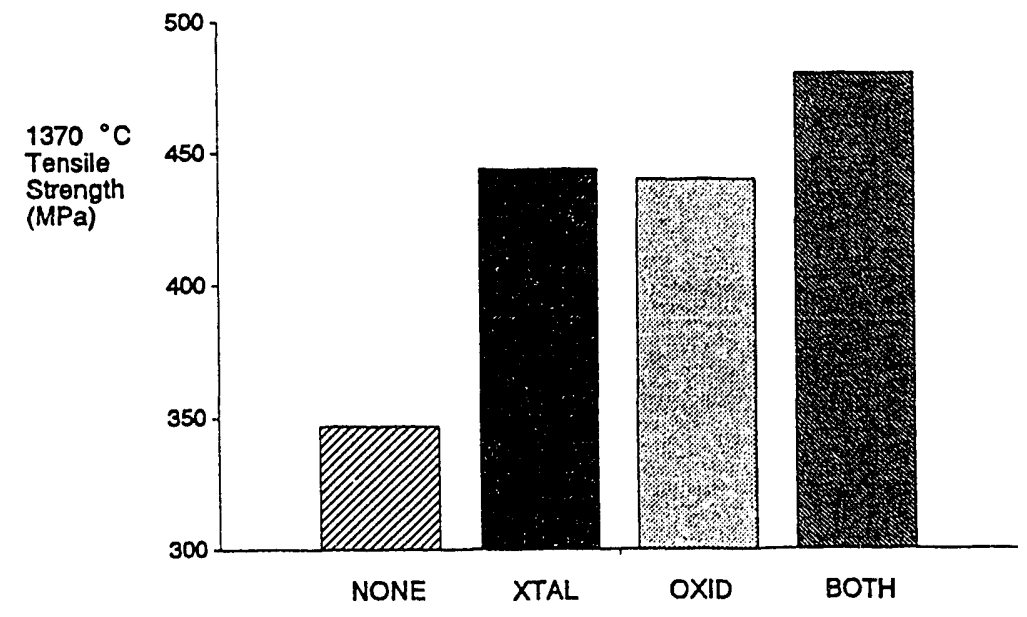

Figure 4-19:

Mean $1370^{\circ} \mathrm{C}$ Tensile Strength as a Function of Heat Treatment condition.

RESIDUAL STRESS IN NSF BARS

An assessment of near surface, machining induced residual stress states in NSF tensile bars was conducted at the Army Material Technology Laboratory. The $x$-ray diffraction approach with $C u R_{a}$ radiation was used. The sin ${ }^{2}$ psi stress measurement technique was employed to provide quantitative data on a total of 13 NSF bars. A number of MOR bars and a $\mathrm{Si}_{3} \mathrm{~N}_{4}$ powder sample were evaluated in the course of the developmental work. This technique provides a stress measurement which represents a bulk volume average, as opposed to point values. In this study, 508 of the diffracted volume is within $22.8 \mu \mathrm{m}$ of the specimen surface. The stresses in the gage section were the primary focus of this work. Longitudinal stresses measured at the center of the gage length were invariably found to be compressive, with magnitudes ranging from 200 to $400 \mathrm{MPa}$. Hoop stresses when detectable were found to be tensile with magnitude ranging to $70 \mathrm{MPa}$.

The technique was utilized to establish the dependence of residual 
stress on post-machining heat treatments, grinding methods of different machine shops and forming (injection molding vs. pressure casting) methods. No effect was found on the as-machined stress state after a series of trial heat treatments. Likewise, no significant differences in residual stress were found in the assessment of specimens machined at three machine shops. A difference was found when comparing injection molded and pressure cast final machined specimens. The cast specimens were found to have a higher level of compressive stress than the injection molded specimens, $350 \mathrm{MPa}$ vs. $245 \mathrm{MPa}$. This could be a result of the green density gradients inherent to the casting process, which cause aIP related residual stresses.

In an attempt to measure HIP related residual stresses, one specimen (R-16) was partially machined in the gage section. Residual stress analysis was performed at 8 orientations corresponding to four different depths of machining as well as the as-HIP'ed surface $10^{\circ}$ orientation). No significant difference in residual stress was found after grinding to depths of $0.012,0.048,0.104$ and 0.114 inches. An unexpected result in this study involved the $x$-ray diffraction integrated intensity which was found to increase with depth of machining, Figure 4-20. This integrated intensity is proportional to the 8 B-phase $\mathrm{Si}_{3} \mathrm{~N}_{4}$. As such, the data form a trace of the diminishing $\alpha$-phase of the HIP reaction layer. This result suggests a potentially useful Q.C. method for assessment of reaction layer removal in machining.

\section{MONITORING OF BENDING IN TENSILE TESTING}

Several specimens were strain gaged prior to testing, to study bending during testing and to determine what, if any, effect bending has on strength and reliability data. Each specimen had four gages mounted around the center of the gage section and located every $90^{\circ}$. The amount of bending is calculated in accordance with ASTM Test Method for SharpNotch Tension Testing with Cylindrical specimens (E 602) in the following manner:

$$
\& \text { Bending }=\frac{2\left[\left(g_{1}-g_{3}\right)^{2}+\left(g_{2}-g_{4}\right)^{2}\right]^{1 / 2}}{\left(g_{1}+g_{2}+g_{3}+g_{4}\right)} \times 100
$$

where

$g_{1}, g_{2}, g_{3}$ and $g_{4}$ are the strain gage readings in units of strain.

A Daytronic system 10 DataPAC was used to monitor and record the strain and load data. Initially, 25 tensile specimens were strain gaged and the load-bend data were recorded without any realignment. The measured bending at failure was $<5.58$ for 24 of the 25 specimens while one was at 12.58 . This assured proper alignment of the test fixtures and the validity of the data.

As part of the experiment 508 (predetermined randomly) of a group of 30 specimens were to be realigned if the measured bending at a preload of $6668 \mathrm{~N}$ (1500 lbs.) was > 108. The realignment involves unloading the specimen, twisting the grip/collet load train assembly and reloading the specimen. If the measured bending was still greater than 
preload of $6668 \mathrm{~N}(1500 \mathrm{lbs}$.$) was >108$. The realignment involves unloading the specimen, twisting the grip/collet load train assembly and reloading the specimen. If the measured bending was still greater than 108, the realignment was performed a second time and the specimen was tested to failure regardless of the measured preload bending. Loaddisplacement curves have a "knee" in the curve at about $5556 \mathrm{~N}(1250$ lbs.) where the copper collets deform into the buttonhead radius. Therefore the preload was chosen to be higher than $5556 \mathrm{~N}$ to monitor the "preload" bending.

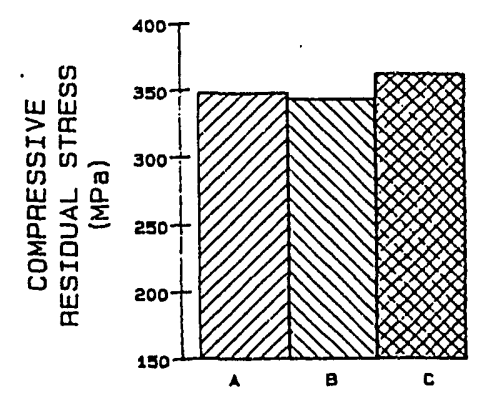

MACHINING PROCEDURE

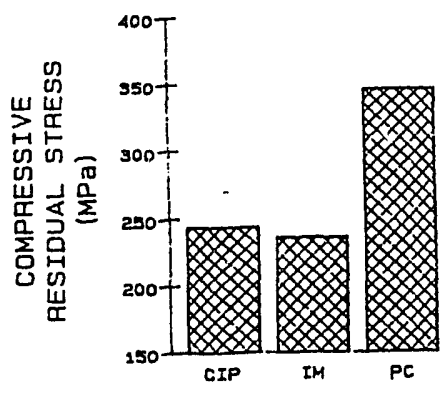

FOAMING METHOD

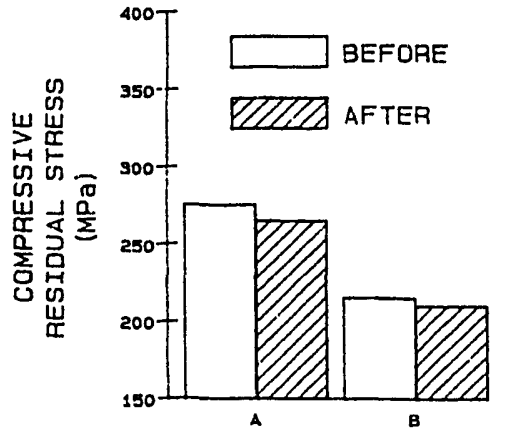

HEAT TREATMENT

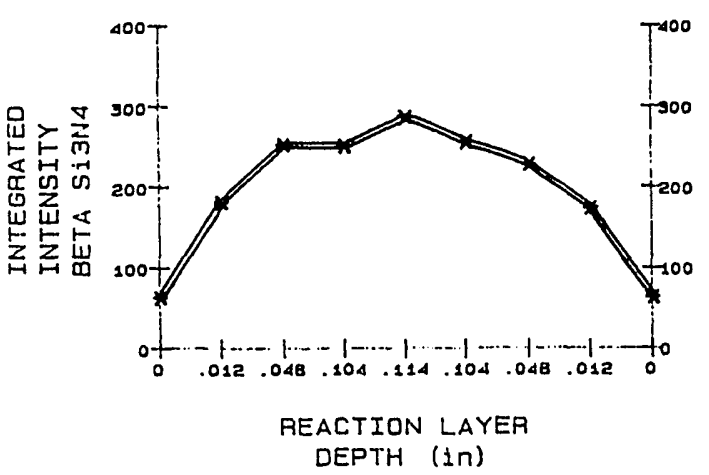

DEPTH $(1 n)$

Figure 4-20: X-ray Diffraction Residual Stress Results

Figure 4-21 shows representative load-bend curves for strain-gaged specimens. A typical curve shows an increase in bending during initial loading but as the collets seat themselves the bending begins to decrease. The Figure shows that the range of bending at the preload level $(6668 \mathrm{~N})$ is 4 to 168 , and in all cases the bending at failure is S 68. For two of the specimens for which realignment took place, the best bending achieved at the preload was 128 , but still resulted in low (28) bending at failure. However, as shown in Figure 4-22, and consistent with the decreasing bending level after preload, there appears to be a trend that the higher strength specimens experience a lower bending at failure. Also noteworthy is that the specimens that had a realignment performed have a lower $\&$ bending at failure than those without a realignment. 
specimen. Two Weibull Moduli were calculated. The first modulus was determined from the standard fracture stress of each specimen (load/area) and was equal to 10.04. A second or predicted fracture stress was determined as the product of the maximum strain times young's Modulus $(310 \mathrm{GPa})$. A Weibull analysis was performed using the predicted strengths giving a value of 10.27. For practical purposes the difference is negligible. It was therefore concluded that 1008 strain gaging is not required. However, a periodic sampling using a strain gaged specimen to check load train alignment was conducted.

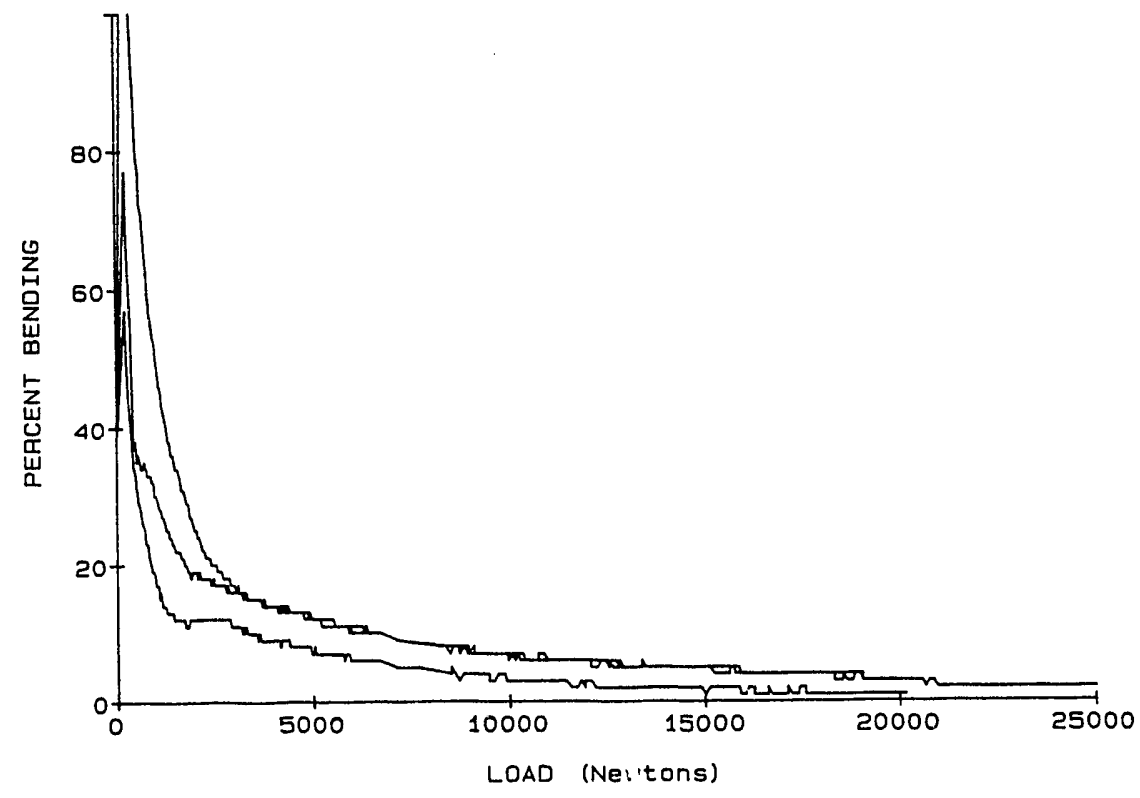

Figure 4-21: Typical Load vs. Benling curves for strain Gaged Tensile specimens

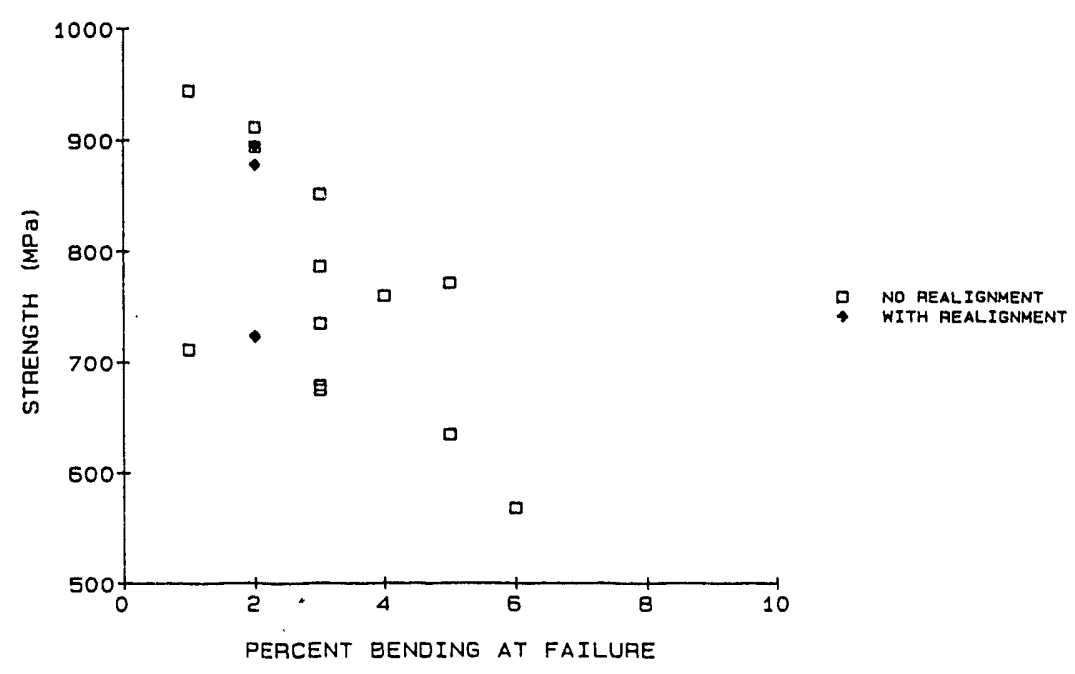

Figure 4-22: Bending at Failure vs. Tensile Strength 
REFERENCES

1. M.G. Jenkins, M.K. Ferber, R.I. Martin et.al., "Study and Analysis of the stress state in a Ceramic, Buttonhead, Tensile Specimen", Oak Ridge National Laboratory, TM-11767, Sept. 1991.

2. P.R. Khandelwal and D.I. Vaccari, "Life Prediction Methodology of Ceramic Engine Components", Procs. Annual Automotive Technology Development CCM, Dearborn, MI, Oct. 28-31, 1991, SAE, 1992, 253260 .

3. A.E. Pasto, S. Natansohn et.al., "Development of Improved Processing Methods for High Reliability Structural Ceramics for Advanced Heat Engines", Oak Ridge National Laboratory Rpt ORNL/Sub/89-SD548/1, July 1992.

4. P. Chantikul, et.al., "A Critical Evaluation of Indentation Techniques for Measuring Fracture Toughness: II, Strength Method", J. Amer. Ceram. Soc., 64, 9, 539-543, 1981.

5. N.I. Becht et.al., "Effects of the Environment on the Mechanical Behavior of Ceramics, Procs. Annual Automotive Technology Development Contractors Coordination Meeting, Dearborn, MI, October 22-25, 1990, SAE, 1991, 199-212.

6. D.M. Tracey, "3D Elastic Singularity Element for Evaluation of $R$ Along an Arbitrary Crack Front," Int'l J. Fracture, 2, pp.340-343, 1973.

7. H.L. Ewalds and R.J.H. Wanhill, Fracture Mechanics, Edward Arnold Publishers, Ltd., Baltimore, MD, pp 42-43, 1984 . 
APPENDIX 5: TENSILE STRENGTH - FRACTOGRAPHY DATABASE

Table 5-1: NCX-5102 Database summary

\begin{tabular}{|c|c|c|c|c|}
\hline \multicolumn{2}{|c|}{$\begin{array}{l}\text { Failure } \\
\text { Origin }\end{array}$} & $\begin{array}{r}\text { Average } \\
\text { (MPa) }\end{array}$ & $\begin{array}{l}\text { std. Dev } \\
\text { (MPa) }\end{array}$ & Count \\
\hline \multicolumn{2}{|c|}{ A11 } & 997 & 115 & 320 \\
\hline \multirow[t]{3}{*}{ By } & Location & & & \\
\hline & surface (s) & 1001 & 107 & 247 \\
\hline & volume (V) & 984 & 138 & 73 \\
\hline \multirow[t]{8}{*}{ By } & Type & & & \\
\hline & Extrinsic & 984 & 133 & 150 \\
\hline & Machining $(\mathrm{M})$ & 999 & 122 & 134 \\
\hline & Inclusion (I) & 865 & 162 & 16 \\
\hline & Intrinsic & 1008 & 96 & 170 \\
\hline & Amorphous (Am) & 1005 & 88 & 107 \\
\hline & Microporosity (Mp) & 1021 & 106 & 55 \\
\hline & Unknown (U) & 960 & 118 & 8 \\
\hline
\end{tabular}

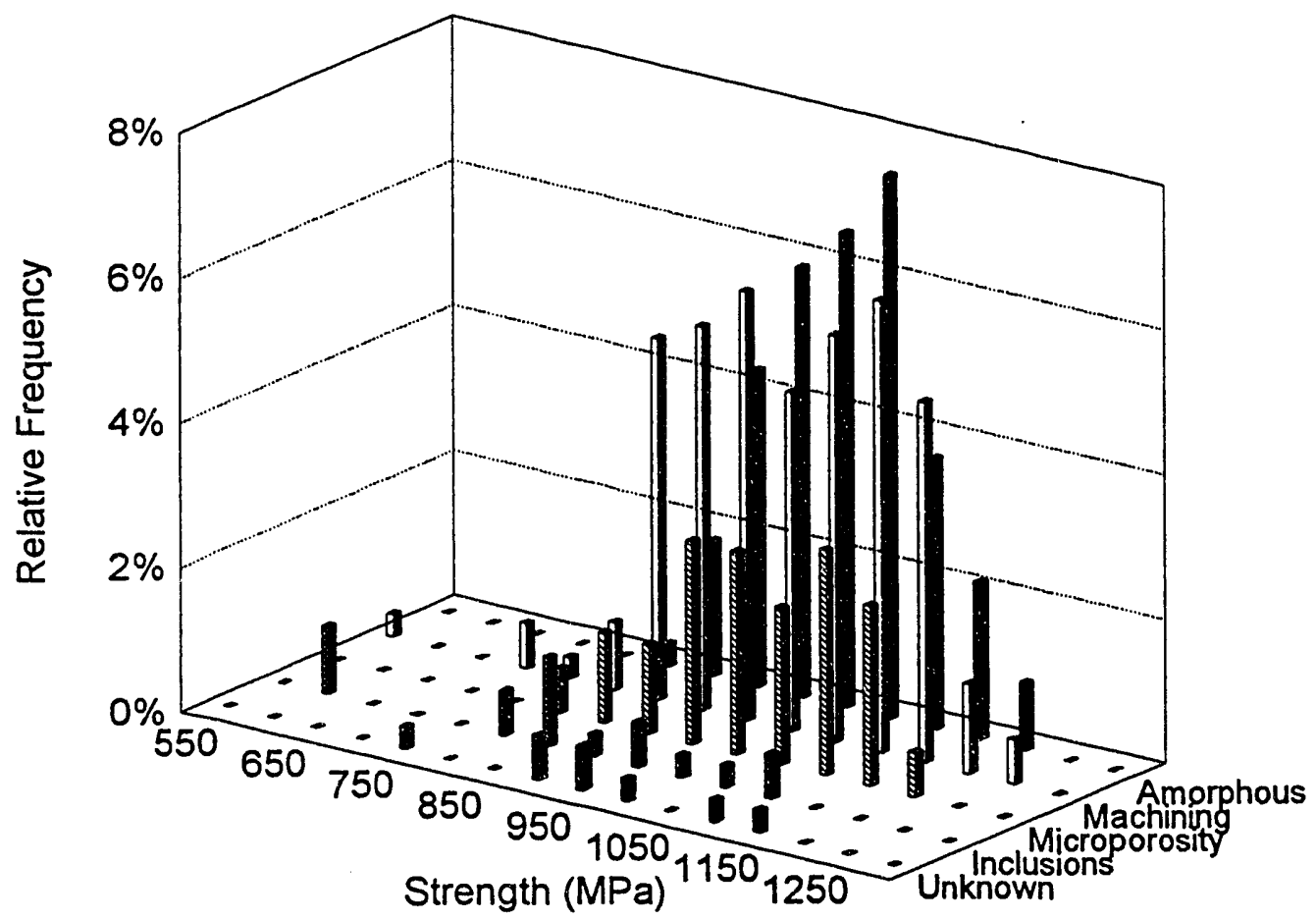

Figure 5-1: Tensile strength Data by Flaw Type 
117

Table 5-2: NCX-5102 Database

$\begin{array}{rrrr}\text { Rank } & \begin{array}{c}\text { Tensile } \\ \text { Strength } \\ \text { (MPa) }\end{array} & \text { Flaw } & \text { Flaw } \\ & & \text { Type }\end{array}$

\begin{tabular}{|c|c|c|c|c|}
\hline 1 & L-169 & 540 & $\mathbf{s}$ & $\mathbf{M}$ \\
\hline 2 & L-91 & 578 & $\mathbf{v}$ & $I$ \\
\hline 3 & $J-121$ & 606 & $\mathbf{v}$ & I \\
\hline 4 & $M-137$ & 619 & v & I \\
\hline 5 & L-97 & 679 & $\mathbf{s}$ & $\mathbf{M}$ \\
\hline 6 & $M-158$ & 706 & $\mathbf{s}$ & $\mathbf{M}$ \\
\hline 7 & $M-159$ & 740 & $\mathbf{v}$ & $\mathbf{u}$ \\
\hline 8 & I-168 & 753 & $\mathbf{s}$ & $\mathbf{M}$ \\
\hline 9 & $M-138$ & 779 & $\mathbf{v}$ & I \\
\hline 10 & L-170 & 786 & $\mathbf{v}$ & Mp \\
\hline 11 & $J-21$ & 809 & $\mathrm{~V}$ & $I$ \\
\hline 12 & H-25 & 812 & $\mathbf{s}$ & M \\
\hline 13 & $I-125$ & 813 & $\mathbf{v}$ & Mp \\
\hline 14 & L-37 & 816 & $\mathbf{s}$ & $\mathbf{M}$ \\
\hline 15 & $8-6$ & 822 & $\mathbf{s}$ & $\mathrm{Am}$ \\
\hline 16 & $I-132$ & 822 & $\mathbf{s}$ & $\mathbf{M}$ \\
\hline 17 & $M-75$ & 826 & $\mathbf{s}$ & $\mathbf{M}$ \\
\hline 18 & H-163 & 834 & $\mathbf{s}$ & An \\
\hline 19 & $I-10$ & 834 & $\mathbf{s}$ & $\mathrm{Am}$ \\
\hline 20 & $\mathrm{H}-108$ & 835 & $\mathbf{v}$ & I \\
\hline 21 & $I-68$ & 836 & $v$ & Mp \\
\hline 22 & $I-144$ & 837 & $\mathbf{s}$ & $M$ \\
\hline 23 & L-59 & 839 & $\mathbf{s}$ & Am \\
\hline 24 & H-89 & 843 & $\boldsymbol{v}$ & Mp \\
\hline 25 & H-99 & 847 & $\mathbf{s}$ & $M$ \\
\hline 26 & $M-56$ & 853 & $\mathbf{v}$ & Mp \\
\hline 27 & $M-55$ & 855 & $\mathbf{v}$ & Mp \\
\hline 28 & B-97 & 856 & $\mathbf{v}$ & I \\
\hline 29 & L-117 & 857 & $\mathbf{s}$ & $\mathbf{M}$ \\
\hline 30 & $I-18$ & 859 & $\mathbf{s}$ & $\mathbf{M}$ \\
\hline 31 & $M-155$ & 860 & $\mathbf{s}$ & $\mathbf{M}$ \\
\hline 32 & L-83 & 861 & $\mathbf{s}$ & $\mathrm{Am}$ \\
\hline 33 & $I-21$ & 862 & $\mathbf{s}$ & Am \\
\hline 34 & $I-82$ & 862 & v & I \\
\hline 35 & $I-123$ & 862 & $\mathbf{s}$ & $\mathbf{M}$ \\
\hline 36 & $M-3$ & 863 & $\mathbf{s}$ & $\mathbf{M}$ \\
\hline 37 & $I-35$ & 865 & $\mathbf{s}$ & $\mathbf{M}$ \\
\hline 38 & $\mathrm{~J}-81$ & 866 & v & $I$ \\
\hline 39 & $J-45$ & 866 & $\mathbf{s}$ & $\mathbf{M}$ \\
\hline 40 & $I-94$ & 868 & $\mathbf{s}$ & $\mathbf{M}$ \\
\hline 41 & $J-89$ & 870 & $\mathbf{s}$ & $\mathrm{Am}$ \\
\hline 42 & $I-22$ & 871 & $\mathbf{s}$ & $M$ \\
\hline 43 & $M-16$ & 871 & $\mathbf{s}$ & $\mathbf{M}$ \\
\hline 44 & $I-17$ & 871 & $\mathbf{s}$ & $\mathbf{M}$ \\
\hline 45 & $13-82$ & 874 & $\mathbf{s}$ & ii \\
\hline
\end{tabular}


118

\begin{tabular}{|c|c|c|c|c|}
\hline 46 & $I-23$ & 875 & $\mathbf{s}$ & $\mathbf{M}$ \\
\hline 47 & $I-72$ & 877 & $\mathbf{v}$ & Mp \\
\hline 48 & $M-76$ & 878 & $\mathbf{s}$ & Am \\
\hline 49 & $J-71$ & 878 & $\mathbf{s}$ & $\mathbf{M}$ \\
\hline 50 & $I-7$ & 879 & $\mathbf{s}$ & $\mathbf{M}$ \\
\hline 51 & $M-69$ & 882 & $\mathbf{s}$ & $\mathbf{M}$ \\
\hline 52 & $M-91$ & 883 & $\mathbf{s}$ & Am \\
\hline 53 & $I-100$ & 883 & $\mathbf{s}$ & An \\
\hline 54 & $I-15$ & 884 & $\mathbf{s}$ & $\mathbf{M}$ \\
\hline 55 & $L-180$ & 886 & $\mathbf{s}$ & Am \\
\hline 56 & $I-15$ & 889 & $\mathbf{s}$ & Am \\
\hline 57 & $M-67$ & 893 & $\mathbf{s}$ & Am \\
\hline 58 & $I-156$ & 893 & $\mathbf{s}$ & Am \\
\hline 59 & M-11 & 893 & $\mathbf{s}$ & $\mathbf{M}$ \\
\hline 60 & $M-86$ & 894 & $\mathbf{s}$ & $\mathbf{M}$ \\
\hline 61 & $I-84$ & 897 & $\mathbf{s}$ & $\mathbf{M}$ \\
\hline 62 & $I-32$ & 898 & $\mathbf{s}$ & $\mathbf{M}$ \\
\hline 63 & $\Sigma-52$ & 899 & $\mathbf{s}$ & $\mathbf{M}$ \\
\hline 64 & B-8 & 900 & $\mathbf{v}$ & $\mathrm{Fe}$ \\
\hline (i) 5 & $\mathrm{~B}-40$ & 901 & $\mathbf{s}$ & Am \\
\hline 66 & $I-104$ & 903 & $\mathbf{s}$ & $\mathbf{M}$ \\
\hline 67 & $I-97$ & 904 & $\mathbf{s}$ & $I$ \\
\hline 68 & $I-26$ & 905 & $\mathbf{s}$ & Am \\
\hline 69 & $I-38$ & 905 & $\mathbf{s}$ & $\mathbf{M}$ \\
\hline 76 & M-33 & 906 & $\mathbf{v}$ & Mp \\
\hline 71 & $M-141$ & 907 & $\mathbf{S}$ & $\mathbf{M}$ \\
\hline 72 & $I-94$ & 908 & $\mathbf{v}$ & Mp \\
\hline 73 & $M-68$ & 917 & $\mathbf{s}$ & $\mathbf{M}$ \\
\hline 74 & $J-47$ & 918 & $\mathbf{v}$ & $\mathrm{Mp}$ \\
\hline 75 & $M-57$ & 919 & $\mathbf{s}$ & $\mathbf{M}$ \\
\hline 76 & $M-144$ & 920 & $\mathbf{s}$ & $\mathbf{M}$ \\
\hline 77 & $I-4$ & 920 & $\mathbf{s}$ & $\mathbf{M}$ \\
\hline 78 & $\mathrm{~J}-107$ & 921 & $\mathbf{s}$ & $\mathrm{Am}$ \\
\hline 79 & $x-122$ & 921 & $\mathbf{s}$ & $\mathrm{Am}$ \\
\hline 80 & $L-85$ & 923 & $\mathbf{s}$ & Am \\
\hline 81 & B-152 & 924 & $\mathbf{S}$ & Am \\
\hline 82 & $4-21$ & 924 & $\mathbf{s}$ & Am \\
\hline 83 & $M-18$ & 925 & $\mathbf{s}$ & $\mathbf{M}$ \\
\hline 84 & $M-153$ & 925 & $\mathbf{s}$ & $\mathbf{U}$ \\
\hline 85 & $M-27$ & 927 & $\mathbf{S}$ & $\mathbf{M}$ \\
\hline 86 & B-42 & 927 & $\mathbf{s}$ & Am \\
\hline 87 & $L-74$ & 927 & $\mathbf{s}$ & Am \\
\hline 88 & $\mathrm{~K}-77$ & 930 & $\mathbf{v}$ & $\mathrm{Mp}$ \\
\hline 89 & $I-173$ & 931 & $\mathbf{v}$ & $I$ \\
\hline 90 & $I-136$ & 931 & $\mathbf{v}$ & Mp \\
\hline 91 & B-105 & 931 & $\mathbf{s}$ & Am \\
\hline 92 & $I-108$ & 936 & $\mathbf{s}$ & Am \\
\hline 93 & $M-140$ & 936 & $\mathbf{s}$ & Am \\
\hline 94 & $L-17$ & 936 & $\mathbf{s}$ & $\mathbf{M}$ \\
\hline \$5 & $J-27$ & 536 & $\ddot{\mathbf{v}}$ & $\ddot{\mathbf{I}}$ \\
\hline 96 & $\mathbf{M}-90$ & 939 & $\mathbf{s}$ & $\mathbf{M}$ \\
\hline 97 & M-96 & 941 & $\mathbf{s}$ & Am \\
\hline
\end{tabular}


119

\begin{tabular}{|c|c|c|c|c|}
\hline 98 & B-7 & 941 & $\mathbf{s}$ & Am \\
\hline 99 & $J-120$ & 943 & $\mathbf{s}$ & Am \\
\hline 100 & $M-29$ & 944 & $\mathbf{s}$ & $\mathbf{M}$ \\
\hline 101 & $M-54$ & 945 & $\mathbf{s}$ & Am \\
\hline 102 & M-39 & 945 & $\mathbf{s}$ & $\mathbf{M}$ \\
\hline 103 & $\mathrm{H}-13$ & 945 & $\mathbf{s}$ & $\mathbf{M}$ \\
\hline 104 & $J-108$ & 946 & $\mathbf{s}$ & Am \\
\hline 105 & $I-35$ & 947 & $\mathbf{s}$ & Am \\
\hline 106 & $M-92$ & 948 & $\mathbf{s}$ & Am \\
\hline 107 & $M-147$ & 948 & $\mathbf{s}$ & $\mathbf{M}$ \\
\hline 108 & $I-24$ & 949 & $\mathbf{s}$ & Am \\
\hline 109 & $I-47$ & 949 & $\mathbf{v}$ & $\mathrm{Mp}$ \\
\hline 110 & $M-6$ & 949 & $\mathbf{v}$ & $\mathrm{Mp}$ \\
\hline 111 & $M-22$ & 951 & $\mathbf{s}$ & $\mathbf{M}$ \\
\hline 112 & $M-53$ & 952 & $\mathbf{s}$ & Am \\
\hline 113 & $\mathrm{~J}-106$ & 952 & $\mathbf{S}$ & $\mathrm{Am}$ \\
\hline 114 & $\mathrm{~J}-59$ & 954 & $\mathbf{s}$ & $\mathrm{Am}$ \\
\hline 115 & $\mathrm{~J}-169$ & 954 & $\mathbf{v}$ & Mp \\
\hline 116 & $I-56$ & 955 & $\mathbf{v}$ & $\mathrm{Mp}$ \\
\hline 117 & $J-153$ & 955 & $\mathbf{s}$ & $\mathbf{M}$ \\
\hline 118 & $M-38$ & 959 & $\mathbf{s}$ & $\mathbf{M}$ \\
\hline 119 & $J-115$ & 960 & $\mathbf{v}$ & Mp \\
\hline 120 & $I-105$ & 961 & $\mathbf{v}$ & Mp \\
\hline 121 & $L-120$ & 961 & $\mathbf{s}$ & $M$ \\
\hline 122 & $I-143$ & 962 & $\mathbf{s}$ & $\mathbf{M}$ \\
\hline 123 & $M-44$ & 964 & $\mathbf{s}$ & $\mathbf{M}$ \\
\hline 124 & $\mathrm{~B}-141$ & 966 & $\mathbf{s}$ & $\mathbf{M}$ \\
\hline 125 & $I-83$ & 966 & $\mathbf{s}$ & Am \\
\hline 126 & $I-16$ & 968 & $\mathbf{s}$ & Am \\
\hline 127 & $\mathrm{~J}-105$ & 970 & $\mathbf{v}$ & Mp \\
\hline 128 & $\mathrm{~J}-48$ & 971 & $\mathbf{s}$ & $\mathbf{M}$ \\
\hline 129 & B-109 & 972 & $\mathbf{s}$ & $\mathbf{M}$ \\
\hline 130 & $I-122$ & 973 & $\mathbf{s}$ & Am \\
\hline 131 & $M-71$ & 973 & $\mathbf{s}$ & $\mathbf{M}$ \\
\hline 132 & $I-88$ & 973 & $\mathbf{s}$ & $\mathbf{M}$ \\
\hline 133 & $\mathrm{~J}-20$ & 974 & $\mathbf{v}$ & $I$ \\
\hline 134 & $M-152$ & 974 & $\mathbf{s}$ & $\mathbf{U}$ \\
\hline 135 & $J-54$ & 975 & $\mathbf{s}$ & $\mathbf{M}$ \\
\hline 136 & $I-131$ & 976 & $\mathbf{s}$ & An \\
\hline 137 & $I-3$ & 978 & $\mathbf{s}$ & $\mathbf{M}$ \\
\hline 138 & $I-89$ & 978 & $\mathbf{s}$ & Am \\
\hline 139 & L-179 & 979 & $\mathbf{s}$ & $\mathbf{M}$ \\
\hline 140 & $M-134$ & 979 & $\mathbf{v}$ & $I$ \\
\hline 141 & $\mathrm{~B}-136$ & 982 & $\mathbf{V}$ & Mp \\
\hline 142 & $\mathrm{~J}-109$ & 984 & $\mathbf{s}$ & Am \\
\hline 143 & $\mathrm{H}-140$ & 984 & $\mathbf{s}$ & $\mathbf{M}$ \\
\hline 144 & M-94 & 985 & $\mathbf{s}$ & $\mathbf{M}$ \\
\hline 145 & $L-33$ & 985 & $\mathbf{s}$ & Am \\
\hline 146 & $J-63$ & 986 & $\mathbf{s}$ & Am \\
\hline $14 ?$ & $M-85$ & 986 & $\mathbf{S}$ & Am \\
\hline 148 & $\mathrm{~B}-52$ & 987 & $\mathbf{s}$ & Am \\
\hline 149 & $\mathrm{H}-23$ & 987 & $\mathbf{s}$ & Am \\
\hline
\end{tabular}




\begin{tabular}{|c|c|c|c|c|}
\hline 150 & I-114 & 988 & $\mathbf{s}$ & $\mathbf{M}$ \\
\hline 151 & $M-45$ & 988 & $\mathbf{s}$ & $\mathbf{M}$ \\
\hline 152 & L-54 & 988 & $\mathbf{v}$ & $\mathrm{Mp}$ \\
\hline 153 & B-151 & 989 & $\mathbf{s}$ & Am \\
\hline 154 & $R-94$ & 989 & $\mathbf{s}$ & Am \\
\hline 155 & $J-41$ & 991 & $\mathbf{s}$ & $\mathbf{M}$ \\
\hline 156 & $M-64$ & 991 & $\mathbf{s}$ & $\mathbf{M}$ \\
\hline 157 & I-139 & 995 & $\mathbf{s}$ & Am \\
\hline 158 & L-122 & 996 & $\mathbf{s}$ & $\mathrm{Am}$ \\
\hline 159 & $J-83$ & 996 & $\mathbf{s}$ & Mp \\
\hline 160 & $M-21$ & 996 & $\mathbf{s}$ & $M$ \\
\hline 161 & $x-46$ & 996 & $\mathbf{s}$ & Am \\
\hline 162 & L-96 & 998 & $\mathbf{s}$ & Am \\
\hline 163 & $M-133$ & 999 & $\mathbf{s}$ & Am \\
\hline 164 & $M-139$ & 1000 & $\mathbf{s}$ & $\mathbf{M}$ \\
\hline 165 & $J-64$ & 1000 & $\mathbf{s}$ & An \\
\hline 166 & $I-78$ & 1000 & v & Mp \\
\hline 167 & $I-60$ & 1002 & $\mathbf{s}$ & 0 \\
\hline 168 & $M-70$ & 1003 & $\mathbf{s}$ & $\mathbf{M}$ \\
\hline 169 & $I-43$ & 1006 & $\mathbf{v}$ & Mp \\
\hline 170 & $J-132$ & 1006 & $\mathbf{v}$ & $\mathrm{Mp}$ \\
\hline 171 & B-15 & 1007 & $\mathbf{s}$ & Am \\
\hline 172 & $M-2$ & 1008 & v & $\mathrm{Mp}$ \\
\hline 173 & $I-48$ & 1010 & $\mathbf{s}$ & $M$ \\
\hline 174 & $I-106$ & 1011 & $\mathbf{s}$ & Am \\
\hline 175 & L-143 & 1014 & $\mathbf{v}$ & Mp \\
\hline 176 & $M-59$ & 1014 & $\mathbf{s}$ & $\mathbf{M}$ \\
\hline 177 & $M-37$ & 1015 & $\mathbf{s}$ & $M$ \\
\hline 178 & B-134 & 1019 & $\mathbf{s}$ & Am \\
\hline 179 & $M-78$ & 1023 & $\mathbf{s}$ & Am \\
\hline 180 & I-5 & 1023 & $\mathbf{v}$ & $\mathrm{Mp}$ \\
\hline 181 & L-10 & 1025 & $\mathbf{s}$ & $\mathbf{M}$ \\
\hline 182 & $M-26$ & 1025 & $\mathbf{s}$ & Am \\
\hline 183 & $y-65$ & 1025 & $\mathbf{s}$ & $\mathbf{M}$ \\
\hline 184 & B-43 & 1027 & $\mathbf{s}$ & $M$ \\
\hline 185 & $M-62$ & 1029 & $\mathbf{s}$ & Am \\
\hline 186 & $M-151$ & 1030 & $\mathbf{s}$ & $\mathbf{M}$ \\
\hline 187 & $J-38$ & 1032 & $\mathbf{s}$ & Am \\
\hline 188 & $M-142$ & 1032 & $\mathbf{s}$ & Am \\
\hline 189 & $M-143$ & 1032 & $\mathbf{s}$ & $\mathbf{M}$ \\
\hline 190 & M-9 & 1032 & $\mathbf{v}$ & Mp \\
\hline 191 & $\mathrm{~J}-122$ & 1033 & $\mathbf{s}$ & $M$ \\
\hline 192 & B-58 & 1033 & $\mathbf{s}$ & $M$ \\
\hline 193 & $\mathrm{~B}-48$ & 1034 & $\mathbf{s}$ & $M$ \\
\hline 194 & B-62 & 1034 & $\mathbf{s}$ & Am \\
\hline 195 & H-96 & 1035 & $\mathbf{s}$ & $\mathbf{M}$ \\
\hline 196 & R-8 & 1036 & v & Mp \\
\hline 197 & B-59 & 1037 & $\mathbf{v}$ & Mp \\
\hline 198 & $I-34$ & 1037 & $\mathbf{s}$ & $M$ \\
\hline 199 & $I-124$ & 1040 & $\mathbf{s}$ & Am \\
\hline 200 & L-75 & 1041 & $\mathbf{s}$ & Am \\
\hline 201 & $I-90$ & 1041 & $\mathbf{s}$ & Am \\
\hline
\end{tabular}


121

\begin{tabular}{|c|c|c|c|c|}
\hline 202 & $I-39$ & 1042 & $\mathbf{s}$ & $\mathbf{M}$ \\
\hline 203 & $L-12$ & 1044 & $\mathbf{s}$ & Am \\
\hline 204 & $I-121$ & 1044 & $\mathbf{s}$ & Am \\
\hline 205 & $I-64$ & 1045 & $\mathbf{s}$ & Am \\
\hline 206 & $M-61$ & 1046 & $\mathbf{s}$ & Am \\
\hline 207 & $\mathrm{~J}-114$ & 1046 & $\mathbf{s}$ & Am \\
\hline 208 & $I-103$ & 1050 & $\mathbf{s}$ & $\mathbf{M}$ \\
\hline 209 & $M-161$ & 1051 & $\mathbf{s}$ & $\mathbf{M}$ \\
\hline 210 & $I-41$ & 1054 & $\mathbf{s}$ & $\mathbf{M}$ \\
\hline 211 & $M-42$ & 1054 & $\mathbf{s}$ & Am \\
\hline 212 & H-165 & 1056 & $\mathbf{s}$ & Am \\
\hline 213 & $M-24$ & 1057 & $\mathbf{V}$ & $\mathrm{Mp}$ \\
\hline 214 & $I-84$ & 1058 & $\mathbf{s}$ & $\mathbf{M}$ \\
\hline 215 & $J-159$ & 1059 & $\mathbf{s}$ & $\mathbf{M}$ \\
\hline 216 & $I-66$ & 1059 & $\mathbf{v}$ & $I$ \\
\hline 217 & $I-14$ & 1060 & $\mathbf{s}$ & $\mathrm{Am}$ \\
\hline 218 & $I-54$ & 1061 & $\mathbf{s}$ & Am \\
\hline 219 & $J-91$ & 1061 & $\mathbf{s}$ & Am \\
\hline 220 & $J-44$ & 1062 & $\mathbf{v}$ & $M p$ \\
\hline 221 & $\mathrm{~B}-50$ & 1062 & $\mathbf{s}$ & Am \\
\hline 222 & $J-78$ & 1064 & $\mathbf{v}$ & $M p$ \\
\hline 223 & M-66 & 1064 & $\mathbf{s}$ & Am \\
\hline 224 & $I-2$ & 1065 & $\mathbf{s}$ & Am \\
\hline 225 & $I-44$ & 1065 & $\mathbf{s}$ & Am \\
\hline 226 & B-93 & 1067 & $\mathbf{s}$ & $\mathbf{M}$ \\
\hline 227 & $I-95$ & 1068 & $\mathbf{s}$ & $\mathbf{M}$ \\
\hline 228 & $\mathrm{~K}-86$ & 1068 & $\mathbf{s}$ & Am \\
\hline 229 & $L-157$ & 1070 & $\mathbf{s}$ & $\mathbf{M}$ \\
\hline 230 & $I-29$ & 1071 & $\mathbf{s}$ & Am \\
\hline 231 & $M-80$ & 1072 & $\mathbf{s}$ & Am \\
\hline 232 & M-5 & 1072 & $\mathbf{v}$ & Mp \\
\hline 233 & $I-56$ & 1076 & $\mathbf{v}$ & $\mathbf{U}$ \\
\hline 234 & L-9 & 1078 & $\mathbf{s}$ & $\mathbf{M}$ \\
\hline 235 & M-93 & 1078 & $\mathbf{s}$ & Am \\
\hline 236 & $\mathrm{~B}-127$ & 1080 & $\mathbf{s}$ & $\mathbf{M}$ \\
\hline 237 & $I-128$ & 1080 & $\mathbf{s}$ & $\mathbf{M}$ \\
\hline 238 & $I-96$ & 1080 & $\mathbf{s}$ & $\mathbf{M}$ \\
\hline 239 & $M-52$ & 1080 & $\mathbf{S}$ & $\mathbf{M}$ \\
\hline 240 & $M-89$ & 1081 & $\mathbf{s}$ & $\mathbf{M}$ \\
\hline 241 & $I-55$ & 1082 & $\mathbf{v}$ & Mp \\
\hline 242 & $\mathrm{~J}-79$ & 1083 & $\mathbf{V}$ & $I$ \\
\hline 243 & $J-93$ & 1085 & $\mathbf{s}$ & $\mathrm{Am}$ \\
\hline 244 & $L-101$ & 1086 & $\mathbf{S}$ & An \\
\hline 245 & $I-105$ & 1087 & $\mathbf{S}$ & Mp \\
\hline 246 & $L-126$ & 1090 & $\mathbf{v}$ & Mp \\
\hline 247 & B-166 & 1090 & $\mathbf{v}$ & $\mathrm{Mp}$ \\
\hline 248 & $J-124$ & 1091 & $\mathbf{s}$ & Am \\
\hline 249 & $I-48$ & 1091 & $\mathbf{s}$ & $\mathbf{M}$ \\
\hline 250 & $I-52$ & 1093 & $\mathbf{s}$ & $\mathbf{M}$ \\
\hline 251 & $L-125$ & 1094 & $\mathbf{S}$ & $\mathbf{M}$ \\
\hline 252 & $\mathrm{~K}-52$ & 1094 & $\mathbf{v}$ & $\mathrm{Mp}$ \\
\hline 253 & $M-20$ & 1098 & $\mathbf{s}$ & Am \\
\hline
\end{tabular}




$$
\|\|
$$


123

$\begin{array}{lllll}306 & M-51 & 1173 & \text { S } & \text { M } \\ 307 & J-170 & 1173 & \text { V } & \text { Mp } \\ 308 & I-32 & 1173 & \text { S } & \text { M } \\ 309 & J-7 & 1175 & \text { V } & \text { Mp } \\ 310 & \text { I-158 } & 1182 & \text { V } & \text { Mp } \\ 311 & I-87 & 1188 & \text { S } & \text { Am } \\ 312 & J-51 & 1193 & \text { V } & \text { Mp } \\ 313 & M-129 & 1194 & \text { S } & \text { Am } \\ 314 & M-87 & 1197 & \text { S } & \text { M } \\ 315 & I-37 & 1198 & \text { S } & \text { M } \\ 316 & I-145 & 1208 & \text { S } & M \\ 317 & I-13 & 1217 & \text { S } & \text { Am } \\ 318 & M-145 & 1218 & \text { S } & M \\ 319 & I-102 & 1234 & \text { S } & M \\ 320 & I-141 & 1237 & \text { S } & M\end{array}$




\section{INTERNAL DISTRIBUTION}

Central Research Library (2)
Document Reference Section
Laboratory Records Department (2)
Laboratory Records, ORNL RC
ORNL Patent Section
M\&C Records office (3)
L. F. Allard, Jr.
I. D. Armstrong
R. I. Beatty
P. F. Becher
T. M. Besmann
P. J. Blau
A. Bleier
E. E. Bloom
K. W. Boling
R. A. Bradley
C. R. Brinkman
V. R. Bullington
R. S. Carlsmith
P. T. Carlson
G. M. Caton
S. J. Chang
R. H. Cooper, Jr.
S. A. David
J. H. DeVan
M. K. Ferber
E. M. Eoust
W. Fulkerson
R. S. Graves
D. L. Greene
H. W. Hayden, Jr.
E. E. Hoffman
C. R. Hubbard
B.

M. A. Janney

D. R. Johnson (5)

F. W. Jones

D. Joslin

R. R. Judkins

M. A. Karnitz

B. I. Keyes

H. D. Kimrey, Jr.

T. G. Kollie

K. C. Iiu

E. L. Long, Jr.

D. J. McGuire

J. R. Merriman

T. A. Nolan

A. E. Pasto

J. I. Rich

C. R. Richmond

G. I. Riner

$J$ M Robbins

G. V. Rogers, Jr.

M. I. Santella

A. C. Schaffhauser

S. Scott

E. J. Soderstrom

D. P. Stinton

R. W. Swindeman

V. J. Tennery

T. N. Tiegs

J. R. Weir, Jr.

B. H. West

S. G. Winslow

J. M. Wyrick

C. S. Yust 
Pioneering Research Information Center E.I. Dupont de Nemours \& Co., Inc. Experimental Station

P.O. Box 80302

Wilmington DE 19880-0302

James H. Adair

University of Florida

Materials Science \& Engineering

317 MAE Bldg.

Gainesville FL 32611-2066

Donald F. Adams

University of Wyoming

Mechanical Engineering Department

P.O. Box 3295

Laramie WY 82071

Jalees Ahmad

AdTech Systems Research Inc.

Solid Mechanics

1342 N. Fairfield Road

Dayton OH 45432-2698

Yoshio Akimune

NISSAN Motor Co., Ltd.

Materials Research Laboratory

1 Natsushima-Cho

Yokosuka 237

JAPAN

Mufit Akinc

Iowa State University

322 Spedding Hall

Ames IA 50011

Ilhan A. Aksay

Princeton University

A313 Engineering Quadrangle

Princeton NJ 08544-5263

R. G. Alexander

BASE

26 Malvern Close

Kettering Northants NN16 AJP

UNITED KINGDOM
Richard L. Allor

Ford Motor Company

Materials Systems Reliability Department

P.O. Box 2053, Room S-2031

Dearborn MI 48121-2053

Joseph E. Amaral

Instron Corporation

Corporate Engineering Office

100 Royale Street

Canton MA 02021

Edward M. Anderson

Aluminum Company of America

North American Industrial Chemical Div.

P.O. Box 300

Bauxite AR 72011

Norman C. Anderson

Ceradyne, Inc.

Ceramic-to-Metal Division

3169 Redhill Avenue

Costa Mesa CA 92626

Don Anson

$\mathrm{BCL}$

Thermal Power Systems

505 King Avenue

Columbus OH 43201-2693

Thomas Arbanas

G.B.C. Materials Corporation

580 Monastery Drive

Latrobe PA 15650-2698

Frank Armatis

3M Company

Building 60-1N-01

St. Paul MN 55144-1000

Everett B. Arnold

Detroit Diesel Corporation

Mechanical Systems Technology

13400 Outer Drive West

Detroit MI 48239-4001 
Richard M. Arons

PA Consulting Group

279 Princeton Road

Hightstown NJ 08550

Bertil Aronsson

Sandvik AB

S-12680

Stockholm Lerkrogsvagen 19

SWEDEN

Dennis Assanis

University of Illinois

Department of Mechanical Engineering

1206 W. Green Street

Urbana IL 61801

V. S. Avva

North Carolina A\&T State University

Department of Mechanical Engineering

Greensboro NC 27411

Patrick Badgley

Adiabatics, Inc.

3385 Commerce Drive

Columbus IN 47201

Sunggi Baik

Pohang Institute of Science \& Technology

P.O. Box 125

Pohang 790-600

KOREA

John M. Bailey

Consultant

Caterpillar, Inc.

P.O. Box 1875

Peoria IL 61656-1875

Bob Baker

Ceradyne, Inc.

3169 Redhill Avenue

Costa Mesa CA 92626

Frank Baker

Aluminum Company of America

Alcoa Technical Center

Alcoa Center PA 15069
J. G. Baldoni

GTE Laboratories, Inc.

40 Sylvan Road

Waltham MA 02254

Clifford P. Ballard

AlliedSignal Aerospace Company

Ceramics Program

P.O. Box 1021

Morristown NJ 07962-1021

B. P. Bandyopadhyay

University of North Dakota

Mechanical Engineering Department

Grand Forks ND 58202-8359

P. M. Barnard

Ruston Gas Turbines Limited

P.O. Box 1

Lincoln LN2 5DJ

ENGLAND

Harold N. Barr

Hittman Corporation

9190 Red Branch Road

Columbia MD 21045

Renald D. Bartoe

Vesuvius McDanel

510 Ninth Avenue

Box 560

Beaver Falls PA 15010-0560

David L. Baty

Babcock \& Wilcox - LRC

P.O. Box 11165

Lynchburg VA 24506-1165

Donald F. Baxter, Jr.

ASM International

Advanced Materials \& Processes

Materials Park OH 44073-0002

M. Brad Beardsley

Caterpillar Inc.

Technical Center Bldg. E

P.O. Box 1875

Peoria IL 61656-1875 
John C. Bell

Shell Research Limited

Thornton Research Centre

P.O. Box 1

Chester CH1 3SH

ENGLAND

Albert H. Bell, III

General Motors Technical Center 30200 Mound Road

Engineering Building/W3 Turbine

Warren MI 48090-9010

M. Bentele

Xamag, Inc.

259 Melville Avenue

Fairfield CT 06430

Larry D. Bentsen

BFGoodrich Company

R\&D Center

9921 Brecksville Road

Brecksville OH 44141

Joseph C. Bentz

ENCERATEC

2525 Sandcrest Drive

Columbus IN 47203

Louis Beregszazi

Defiance Precision Products

P.O. Drawer 428

Defiance OH 43512

Tom Bernecki

Northwestern University

1801 Maple Avenue

Evanston IL 60201-3135

Charles F. Bersch

Institute for Defense Analyses

1801 N. Beauregard Street

Alexandria VA 22311

Ram Bhatt

NASA Lewis Research Center

21000 Brookpark Road

Cleveland $\mathrm{OH} 44135$
Deane I. Biehler

Caterpillar Inc.

Engineering Research Materials

P.O. Box 1875, Bldg. E

Peoria IL 61656-1875

John W. Bjerklie

Consolidated Natural Gas Service Co. Inc.

Research Department

Pittsburgh PA 15222-3199

William D. Bjorndahl

TRW, Inc.

One Space Park

Building 01, Room 2040

Redondo Beach CA 90278

Keith A. Blakely

Advanced Refractory Technologies, Inc.

699 Hertel Avenue

Buffalo NY 14207

Edward G. Blanchard

Netzsch Inc.

119 Pickering Way

Exton PA 19341

Bruce Boardman

Deere and Company Technical Center

3300 River Drive

Moline IL 61265

Russell Bockstedt

Hoechst Celanese Corporation

150 JFK Parkway

Short Hills NJ 07078

M. Boehmer

DLR German Aerospace Research Estab.

Postfach 906058

D-5000 Koln 90

GERMANY

Lawrence P. Boesch

EER Systems Corp.

1593 Spring Hill Road

Vienna VA 22182-2239 
Donald H. Boone

Boone \& Associates

2412 Cascade Drive

Walnut Creek CA 94598-4313

Tom Booth

AlliedSignal, Inc.

AiResearch Los Angeles Division

2525 West 190th Street

Torrance CA 90509-2960

Tibor Bornemisza

Sundstrand Power Systems

4400 Ruffin Road

San Diego CA 92186-5757

J.A.M. Boulet

University of Tennessee

Engineering Science and Mechanics

Knoxville TN 37996-2030

H. Kent Bowen

Massachusetts Institute of Technology

77 Massachusetts Avenue, Room E40-434

Cambridge MA 02139

Leslie J. Bowen

Materials Systems

53 Hillcrest Road

Concord MA 01742

Steven C. Boyce

Air Force Office of Scientific Research

AFOSR/NA Bldg. 410

Bolling AFB DC 20332-6448

Gary L. Boyd

Ceramic Engineering Consulting (CEC)

328 Sneath Way

Alpine CA 91901

Steve Bradley

UOP Research Center

50 E. Algonquin Road

Des Plaines IL 60017-6187
Michael C. Brands

Cummins Engine Company, Inc.

P.O. Box 3005, Mail Code 50179

Columbus IN 47201

Raymond J. Bratton

Westinghouse Science \& Technology Ctr.

1310 Beulah Road

Pittsburgh PA 15235

John J. Brennan

United Technologies Corporation

Silver Lane, MS:24

East Hartford CT 06108

Jeff D. Bright

Ceramatec, Inc.

2425 South 900 West

Salt Lake City UT 84108

Terrence K. Brog

Coors Ceramics Company

4545 McIntyre Street

Golden CO 80403

Gunnar Broman

317 Fairlane Drive

Spartanburg SC 29302

Al Brown

High-Tech Materials Alert

P.O. Box 882

Dayton NJ 08810

Jesse J. Brown

VPI \& SU

Center for Advanced Ceramic Materials

Blacksburg VA 24061-0256

Sherman D. Brown

University of Illinois

Materials Science and Engineering

105 South Goodwin Avenue

Urbana IL 61801 
S. L. Bruner

Ceramatec, Inc.

2425 South 900 West

Salt Lake City UT 84119

Adolfo Brusaferro

Keramont Corporation

4231 S. Fremont Avenue

Tucson AZ 85714

Walter Bryzik

U.S. Army Tank Automotive Command

R\&D Center, Propulsion Systems Div.

Warren MI 48397-5000

S. T. Buljan

GTE Laboratories, Inc.

40 Sylvan Road

Waltham MA 02254

S. J. Burden

2572 Devonwood

Troy MI 48098

Curt V. Burkland

AMERCOM, Inc.

8928 Fullbright Avenue

Chatsworth CA 91311

Bill Bustamante

AMERCOM, Inc.

8928 Fullbright Avenue

Chatsworth CA 91311

Oral Buyukozturk

Massachusetts Institute of Technology

77 Massachusetts Avenue, Room 1-280

Cambridge MA 02139

David A. Caillet

Ethyl Corporation

451 Florida Street

Baton Rouge La 70801

Frederick J. Calnan

Heany Industries, Inc.

249 Briarwood Lane

Scottsville NY 14546
Roger Cannon

Rutgers University

P.O. Box 909

Piscataway NJ 08855-0909

Scott Cannon

P.O. Box 567254

Atlanta GA 30356

Harry W. Carpenter

1844 Fuerte

Fallbrook CA 92028

David Carruthers

Kyocera Industrial Ceramics Company

P.O. Box 2279

Vancouver WA 98668-2279

Calvin H. Carter, Jr.

Cree Research, Inc.

2810 Meridian Parkway

Durham NC 27713

J. David Casey

35 Atlantis Street

West Roxbury MA 02132

Jere G. Castor

J. C. Enterprise

5078 N. 83rd Street

Scottsdale AZ 85250

James D. Cawley

Case Western Reserve University

Materials Science \& Engineering Dept.

Cleveland $\mathrm{OH} 44106$

Thomas C. Chadwick

Den-Mat Corporation

P.O. Box 1729

Santa Maria CA 93456

Ronald H. Chand

Chand Kare Technical Ceramics

2 Coppage Drive

Worcester MA 01603 
Robert E. Chaney

EG\&G Idaho, Inc.

Idaho National Engineering Laboratory

P.O. Box 1625

Idaho Falls ID 83415-3525

Frank C. Chang

U.S. Army Materials Technology Lab

AMTL-EMM

405 Arsenal Street

Watertown MA 02172

Robert M. Chapman

AlliedSignal Aerospace Company

1001 Pennsylvania Avenue, N.W.

Suite 700 South

Washington DC 20004

William Chapman

Williams International Corporation

2280 W. Maple Road

Walled Lake MI 48390-0200

Charlie Chen

LECO Corporation

P.O. Box 211688

Augusta GA 30917

Frank Childs

EG\&G Idaho, Inc.

Idaho National Engineering Laboratory

P.O. Box 1625

Idaho Falls ID 83415-3527

William J. Chmura

Torrington Company

59 Field Street

Torrington CT 06790-4942

Tsu-Wei Chou

University of Delaware

201 Spencer Laboratory

Newark DE 19716
R. J. Christopher

Ricardo Consulting Engineers

Bridge Works

Shoreham-By-Sea West Sussex BN43 5FG

ENGLAND

Joel P. Clark

Massachusetts Institute of Technology

Room 8-409

Cambridge MA 02139

Giorgio Clarotti

Commission of the European Communities

DGXII-C3, MO75, 1-53; 200 Rue de la Loi

B-1049 Brussels

BELGIUM

W. J. Clegg

ICI Advanced Materials

P.O. Box 11

The Heath, Runcorn Cheshire WA7 4QE

ENGLAND

Joseph Cleveland

GTE Products Corporation

Hawes Street

Towanda PA i8848-0504

Gloria M. Collins

ASTM

1916 Race Street

Philadelphia PA 19103

William C. Connors

Sundstrand Aviation Operations

Materials Science \& Engineering Dept.

4747 Harrison Avenue

Rockford IL 61125-7002

John A. Coppola

Carborundum Company

Niagara Falls R\&D Center

P.O. Box 832

Niagara Falls NY 14302 
Normand D. Corbin

Norton Company

Advanced Ceramics

Goddard Road

Northboro MA 01532-1545

Douglas Corey

AlliedSignal, Inc.

2525 West 190th Street, MS:T52

Torrance CA 90504-6099

Keith P. Costello

Chand/Kare Technical Ceramics

2 Coppage Drive

Worcester MA 01603-1252

Ed L. Courtright

Pacific Northwest Laboratory

MS:K3-59

Richland WA 99352

Anna Cox

Mitchell Market Reports

P.O. Box 23

Monmouth Gwent NP5 4YG

UNITED KINGDOM

J. Wesley Cox

BIRL

1801 Maple Avenue

Evanston IL 60201-3135

Art Cozens

Instron Corporation

3414 Snowden Avenue

Long Beach CA 90808

Robert C. Craft

American Ceramic Society, Inc.

735 Ceramic Place

Westerville OH 43081-8720

Mark Crawford

New Technology Week

4604 Monterey Drive

Annandale VA 22003
Richard A. Cree

Markets \& Products, Inc.

P.O. Box 14328

Columbus OH 43214-0328

Les Crittenden

Vesuvius McDanel

Box 560

Beaver Falls PA 15010

William J. Croft

U.S. Army Materials Technology Lab

405 Arsenal Street

Watertown MA 02172

M. J. Cronin

Mechanical Technology, Inc.

968 Albany-Shaker Road

Latham NY 12110

Gary M. Crosbie

Ford Motor Company

1430 Culver Avenue

S-2079, SRL Building

Dearborn MI 48121-4036

Floyd W. Crouse, Jr.

U.S. Department of Energy

Morgantown Energy Technology Center

P.O. Box 880

Morgantown WV 26505

John Cuccio

AlliedSignal, Inc.

Auxiliary Power

P.O. Box 52180, MS:1302-2Q

Phoenix AZ 85072-2180

Raymond A. Cutler

Ceramatec, Inc.

2425 South 900 West

Salt Lake City UT 84119

Stephen C. Danforth

Rutgers University

P.O. Box 909

Piscataway NJ 08855-0909 
Sankar Das Gupta

Electrofuel Manufacturing Co., Ltd.

9 Hanna Avenue

Toronto Ontario MGK-1W8

CANADA

Charles Davis

Sverdrup Technology, Inc. MSFC

620 Discovery Drive

Huntsville AL 35806

Frank Davis

AlliedSignal Aerospace Company

7550 Lucerne Drive, \#203

Middleburg Heights $\mathrm{OH} 44130$

Robert F. Davis

North Carolina State University

Materials Engineering Department

P.O. Box 7907

Raleigh NC 27695

Thomas DeAngelis

Carborundum Company

Niagara Falls R\&D Center

P.O. Box 832

Niagara Falls NY 14302

George DeBell

Ford Motor Company

Material Systems Reliability Dept.

P.O. Box 2053, Room S-2023

Dearborn, MI 48121-2053

Michael DeLuca

AMP-AKZO

West Lane

Aquebogue NY 11931

Gerald L. DePoorter

Colorado School of Mines

Metallurgical \& Materials Engineering

Golden CO 80401

I F. DeRidder

Omni Electro Motive, Inc.

12 Seely Hill Road

Newfield NY 14867
Nick C. Dellow

Materials Technology Publications

40 Sotheron Road

Watford Herts WD1 2QA

UNITED KINGDOM

L. R. Dharani

University of Missouri-Rolla

224 M.E.

Rolla MO 65401

Douglas A. Dickerson

Union Carbide Specialty Powders

1555 Main Street

Indianapolis IN 46224

John Dodsworth

Vesuvius Research \& Development

Technical Ceramics Group

Box 560

Beaver Falls PA 15010

B. Dogan

Institut fur Werkstofforschung

GKSS-Forschungszentrum Geesthacht

Max-Planck-Strasse

D-2054 Geesthacht

GERMANY

Jean-Marie Drapier

FN Moteurs S.A.

Material and Processing

B-4041 Milmort (Herstal)

BELGIUM

Kenneth C. Dreitlein

United Technologies Research Center

Silver Lane

Eas1 Hartford CT 06108

Rob.n A.L. Drew

McG.ll University

3450 University Street

Montr:al Quebec H3A 2A7

CANAL'A 
Winston H. Duckworth

BCL

Columbus Division

505 King Avenue

Columbus OH 43201-2693

Bill Durako

Sundstrand Aviation Operations

P.O. Box 7002

Rockford IL 61125-7002

Ernest J. Duwell

3M Abrasive Systems Division

3M Center

St. Paul MN 55144-1000

Chuck J. Dżiedzic

Coors Ceramics Company

17750 West 32nd Avenue

Golden CO 80401

\section{Robert J. Eagan}

Sandia National Laboratories

Engineered Materials and Processes Ctr.

P.O. Box 5800

Albuquerque NM 871.85-5800

Jeffrey Eagleson

Lanxide Corporation

1001 Connecticut Avenue, N.W.

Washington DC 20036

Harry E. Eaton

United Technologies Corporation

Silver Lane

East Hartford CT 06108

Harvill C. Eaton

Louisiana State University

240 Thomas Boyd Hall

Baton Rouge LA 70803

Christopher A. Ebel

Carborundum Company

Technology Division

P.O. Box 337

Niagar Falls NY 14302
J. J. Eberhardt

U.S. Department of Energy

Office of Transportation Materials

CE-34, Forrestal Building

Washington DC 20585

Jim Edler

Eaton Corporation

26201 Northwestern Highway

P.O. Box 766

Southfield MI 48037

G. A. Eisman

Dow Chemical Company

Ceramics and Advanced Materials

52 Building

Midland MI 48667

William A. Ellingson

Argonne National Laboratory

Materials Science \& Technology Div.

9700 S. Cass Avenue

irgonne IL 60439

William S. Ellis

Machined Ceramics

629 N. Graham Street

Bowling Green KY 42101

Glen B. Engle

Nuclear \& Aerospace Materials Corp.

16716 Martincoit Road

Poway CA 92064

Jeff Epstein

Ceramic Technologies, Inc.

12739 Ashford Knoll

Houston TX 77082

Kenneth A. Epstein

Dow Chemical USA

800 Building

Midland MI 48667

Art Erdemir

Argonne National Laborâtory

9700 S. Cass Avenue

Argonne IL 50439 
E. M. Erwin

Lubrizol Corporation 1819 East 225th Street

Euclid OH 44117

John N. Eustis

U.S. Department of Energy

Industrial Energy Efficiency Division

CE-221, Forrestal Building

Washington DC 20585

W. L. Everitt

Kyocera International, Inc.

8611 Balboa Avenue

San Diego CA 92123

Gordon Q. Evison

332 S. Michigan Avenue

Suite 1730

Chicago IL 60604

John W. Fairbanks

U.S. Department of Energy

Office of Propulsion Systems

CE-322, Forrestal Building

Washington DC 20585

Tim Fawcett

Dow Chemical Company

Advanced Ceramics Laboratory

1776 Building

Midland MI 48674

Robert W. Fawley

Sundstrand Power Systems

Division of Sundstrand Corporation

P.O. Box 85757

San Diego CA 92186-5757

John J. Fedorchak

GTE Products Corporation

Hawes Street

Towanda PA 18848-0504

Jeff T. Fenton

Vista Chemical Company

900 Threadneedle

Houston TX 77079
Larry Ferrell

Babcock \& Wilcox

Old Forest Road

Lynchburg VA 24505

Raymond R. Fessler

BIRL

1801 Maple Avenue

Evanston IL 60201

Ross F. Firestone

Ross Firestone Company

188 Mary Street

Winnetka IL 60093-1520

Sharon L. Fletcher

Arthur D. Little, Inc.

15 Acorn Park

Cambridge MA 02140-2390

M. R. Foley

Norton Company

Advanced Ceramics

Goddard Road

Northboro, MA 01532-1545

Thomas F. Foltz

Textron Specialty Materials

2 Industrial Avenue

Lowell MA 01851

Renee G. Ford

Materials and Processing Report

P.O. Box 72

Harrison NY 10528

John Formica

Supermaterials

2020 Lakeside Avenue

Cleveland $\mathrm{OH} 44114$

Edwin Frame

Southwest Research Institute

P.O. Drawer 28510

San Antonio TX 78284 
Armanet Francois

French Scientific Mission

4101 Reservoir Road, N.W.

Washington DC 20007-2176

R. G. Frank

Technology Assessment Group

10793 Bentley Pass Lane

Loveland OH 45140

David J. Franus

Forecast International

22 Commerce Road

Newtown CT 06470

Marc R. Freedman

NASA Lewis Research Center

21000 Brookpark Road, MS:49-3

Cleveland OH 44135

Douglas Freitag

LTV Missiles Division

P.O. Box 650003, MS:WT-21

Dallas TX 75265

Brian R.T. Frost

Argonne National Laboratory

9700 S. Cass Avenue, Bldg. 900

Argonne IL 60439

Lawrence R. Frost

Instron Corporation

100 Royall Street

Canton MA 02021

George A. Fryburg

Norton/TRW Ceramics

7A-4 Raymond Avenue

Salem NH 03079

Xiren Fu

Shanghai Institute of Ceramics

1295 Ding-xi Road

Shanghai 200050

CHINA
John Gahimer

P.O. Box 1302

Dublin OH 43017

J. P. Gallagher

University of Dayton Research Institute

300 College Park, JPC-250

Dayton OH 45469-0120

Garry Garvey

Golden Technologies Company Inc.

4545 McIntyre Street

Golden CO 80403

Joy A. Garwood

Norton Company

Advanced Ceramics

Goddard Road

Northboro MA 01532-1545

Richard Gates

NIST

Materials Bldg., A-256

Gaithersburg MD 20899

H. Maury Gatewood

Reynolds Metals Company

P.O. Box 27003

Richmond VA 23261

L. J. Gauckler

ETH-Zurich

Sonneggstrasse 5

CH-8092 Zurich 8092

SWITZERLAND

George E. Gazza

U.S. Army Materials Technology Lab

Ceramics Research Division

405 Arsenal Street

Watertown MA 02172-0001

D. Gerster

CEA-DCOM

33 Rue De La Federation

Paris 75015

FRANCE 
John Ghinazzi

Coors Technical Ceramics Company

1100 Commerce Park Drive

Oak Ridge TN 37830

Robert Giddings

General Electric Company

P.O. Box 8

Schenectady NY 12301

A. M. Glaeser

University of California

Lawrence Berkeley Laboratory

Hearst Mining Building

Berkeley CA 94720

Joseph W. Glatz

Naval Air Propulsion Center

Systems Engineering Division

P.O. Box 7176, PE24

Trenton NJ 08628

W. M. Goldberger

Superior Graphite Company

R\&D

2175 E. Broad Street

Columbus OH 43209

Allan E. Goldman

U.S. Graphite, Inc.

907 W. Outer Drive

Oak Ridge TN 37830

Stephen T. Gonczy

Allied Signal Research

P.O. Box 5016

Des Plaines IL 60017

Jeffrey M. Gonzales

GTE Products Corporation

Hawes Street

Towanda PA 18848-0504

Robert J. Gottschall

U.S. Department of Energy

ER-131, MS:G-236

Washington DC 20545
Earl Graham

Cleveland State University

Department of Chemical Engineering

Euclid Avenue at East 24th Street

Cleveland $\mathrm{OH} 44115$

John W. Graham

Astro Met, Inc.

9974 Springfield Pike

Cincinnati OH 45215

William A. Graham

Lanxide Corporation

P.O. Box 6077

Newark DE 19714-6077

Robert E. Green, Jr.

Johns Hopkins University

Materials Science and Engineering

Baltimore MD 21218

Lance Groseclose

General Motors Corporation

Allison Gas Turbine Division

P.O. Box 420, MS:W-5

Indianapolis IN 46206

Thomas J. Gross

U.S. Department of Energy

Transportation Technologies

CE-30, Forrestal Building

Washington DC 20585

Mark F. Gruninger

Union Carbide Corporation

Specialty Powder Business

1555 Main Street

Indianapolis IN 46224

Ernst Gugel

Cremer Forschungsinstitut GmbH\&Co.KG

Oeslauer Strasse 35

D-8633 Roedental 8633

GERMANY 
Donald L. Guile

Corning Glass Works

SP-DV-1-9

Corning NY 14831

John P. Gyekenyesi

NASA Lewis Research Center 21000 Brookpark Road, MS:6-1

Cleveland OH 44135

Nabil S. Hakim

Detroit Diesel Corporation 13400 Outer Drive West

Detroit MI 48239

Philip J. Haley

General Motors Corporation

P.O. Box 420, MS:T12A

Indianapolis IN 46236

Judith Hall

Fiber Materials, Inc.

Biddeford Industrial Park

5 Morin Street

Biddeford ME 04005

Y. Hamano

Kyocera Industrial Ceramics Corp.

5713 E. Fourth Plain Blvd.

Vancouver WA 98661-6857

Y. Harada

IIT Research Institute

10 West 35th Street

Chicago IL 60616

R. A. Harmon

25 Schalren Drive

Latham NY 12110

Norman H. Harris

Hughes Aircraft Company

P.O. Box 800520

Saugus CA 91380-0520
Alan M. Hart

Dow Chemical Company

1776 Building

Midland MI 48674

Pat E. Hart

Battelle Pacific Northwest Laboratories

Ceramics and Polymers Development Sec.

P.O. Box 999

Richland WA 99352

Stephen D. Hartline

Norton Company

Advanced Ceramics

Goddard Road

Northboro MA 01532-1545

Michael H. Haselkorn

Caterpillar In.

Technical Center, Building E

P.O. Box 1875

Peoria IL 61656-1875

N. B. Havewala

Corning Inc.

SP-PR-11

Corning NY 14831

John Haygarth

Teledyne WAA Chang Albany

P.O. Box 460

Albany OR 97321

Norman L. Hecht

University of Dayton Research Institute

300 College Park

Dayton OH 45469-0172

Peter W. Heitman

General Motors Corporation

P.O. Box 420, MS:W-5

Indianapolis IN 46206-0420

Robert W. Hendricks

VPI \& SU

210 Holden Hall

Blacksburg VA 24061-0237 
Wynne Henley

Hertel Cutting Technologies Inc. 1000 Clearview Court

Oak Ridge TN 37830

Thomas L. Henson

GTE Products Corporation

Chemical \& Metallurgical Division

Hawes Street

Towanda PA 18848

Thomas P. Herbell

NASA Lewis Research Center 21000 Brookpark Road, MS:49-3

Cleveland OH 44135

Marlene Heroux

Rolls-Royce, Inc.

2849 Paces Ferry Road, Suite 450

Atlanta GA 30339-3769

Robert L. Hershey

Science Management Corporation 1255 New Hampshire Ave., N.W.

Suite 1033

Washington DC 20036

Hendrik Heystek

Bureau of Mines

Tuscaloosa Research Center

P.O. Box L

University AL 35486

Robert V. Hillery

GE Aircraft Engines

One Neumann Way, M.D. H85

Cincinnati $\mathrm{OH} 45215$

Arthur Hindman

Instron Corporation

100 Royall Street

Canton MA 02021

Jon Hines

American Ceramic Society, Inc.

735 Ceramic Place

Westerville $\mathrm{OH} 43071-8720$
Hans Erich Hintermann

CSEM

Rue Breguet 2

Neuchatel 2000

SWITZERLAND

Shinichi Hirano

Mazda R\&D of North America, Inc.

1203 Woodridge Avenue

Ann Arbor MI 48105

Tommy Hiraoka

NGK Locke, Inc.

1000 Town Center

Southfield MI 48075

John M. Hobday

U.S. Department of Energy

Morgantown Energy Technology Center

P.O. Box 880

Morgantown WV 26507

Clarence Hoenig

Lawrence Livermore National Laboratory

P.O. Box 808, Mail Code L-369

Livermore CA 94550

Thomas Hollstein

Fraunhofer-Institut fur Werkstoffmechanik

Wohlerstrabe 11

D-7800 Freiburg

GERMANY

Richard Holt

National Research Council of Canada

Structures and Materiais Laboratory

Ottawa Ontario K1A OR6

CANADA

Michael Horgan

Materials Engineering Magazine

1100 Superior Avenue

Cleveland OH 44114

Woodie Howe

Coors Technical Ceramics Company

1100 Commerce Park Drive

Oak Ridge TN 37830 
Stephen M. Hsu

NIST

Gaithersburg MD 20899

Hann S. Huang

Argonne National Laboratory

9700 S. Cass Avenue

Argonne IL 60439-4815

Gene Huber

Precision Ferrites \& Ceramics

5576 Corporate Drive

Cypress CA 90630

Harold A. Huckins

Princeton Advanced Technology, Inc.

56 Finley Road

Princeton NJ 08540

Fred R. Huettic

Advanced Magnetics Inc.

45 Corey Lane

Mendham NJ 07945

Brian K. Humphrey

Lubrizol Petroleum Chemicals Co.

3000 Town Center, Suite 1340

Southfield MI 48075-1201

Robert M. Humrick

Dylon Ceramic Technologies

3100 Edgehill Road

Cleveland Heights OH 44118

Lorretta Inglehart

National Science Foundation

Division of Materials Research

1800 "G" Street, N.W., Room 408

Washington DC 20550

Michael S. Inoue

Kyocera International, Inc.

8611 Balboa Avenue

San Diego CA 92123-1580
Osama Jadaan

University of Wisconsin-Platteville

1 University Plaza

Platteville WI 53818

Said Jahanmir

NIST

Materials Science \& Engineering Lab

Gaithersburg MD 20899

Curtis A. Johnson

General Electric Company

P.O. Box 8

Schenectady NY 12301

Sylvia Johnson

SRI International

333 Ravenswood Avenue

Menlo Park CA 94025

Thomas A. Johnson.

Lanxide Corporation

P.O. Box 6077

Newark DE 19714-6077

W. S. John:son

Indiana University

One City Centre, Suite 200

Bloomington IN 47405

Walter F. Jones

Air Force Office of Scientific Research

Bolling Air Force Base

Washington DC 20332-6448

Jill E. Jonkouski

U.S. Department of Energy

9800 S. Cass Avenue

Argonne IL 60439-4899

L. A. Joo

Great Lakes Research Corporation

P.O. Box 1031

Elizabethton TN 37643 
A. David Joseph

SPX Corporation

700 Terrace Point

Muskegon MI 49443

Adam Jostsons

Australian Nuclear Science \& Technology

New Illawarra Road

Lucas Heightss New South Wales

AUSTRALIA

Matthew K. Juneau

Ethyl Corporation

451 Florida Street

Baton Rouge LA 70801

Hartmut Kainer

Didier-Werke AG

Anlagentechnik Wiesbaden

D-62 Wiesbaden

GERMANY

Tom Kalamasz

Norton/TRW Ceramics

7A-4 Raymond Avenue

Salem NH 03079

Lyle R. Kallenbach

Phillips Petroleum

Mail Drop:123AL

Bartlesville OK 74004

Nick Kamiya

Kyocera Industrial Ceramics Corporation 25 Northwest Point Blvd., Suite 450

Elk Grove Village IL 60007

Roy Kamo

Adiabatics, Inc.

3385 Commerce Park Drive

Columbus IN 47201

Chih-Chun Kao

Industrial Technology Research Institute

195 Chung-Hsing Road, Sec. 4

Chutung Hsinchu 31015 R.O.C.

TAIWAN
Keith R. Karasek

AlliedSignal Aerospace Company

50 E. Algonquin Road

Des Plaines IL 60017-5016

Martha R. Kass

U.S. Department of Energy

Oak Ridge Operations

Building 4500N, MS:6269

Oak Ridge TN 37831-6269

Robert E. Kassel

Ceradyne, Inc.

3169 Redhill Avenue

Costa Mesa CA 92626

Allan Katz

Wright Laboratory

Metals and Ceramics Division

Wright-Patterson AFB OH 45433

R. Nathan Katz

Worcester Polytechnic Institute

100 Institute Road

Worcester MA 01609

Tony Kaushal

Detroit Diesel Corporation

13400 Outer Drive West, Speed Code:L-04

Detroit MI 48239

Ted Kawaguchi

Tokai Carbon America, Inc.

375 Park Avenue, Suite 3802

New York NY 10152

Noritsugu Kawashima

TOSHIBA Corporation

4-1 Ukishima-Cho

Kawasaki-Ku Kawasaki 210

JAPAN

Lisa Kempfer

Penton Publishing

1100 Superior Avenue

Cleveland $\mathrm{OH} 44114-2543$ 
Frederick L. Kennard, III General Motors Corporation

AC Rochester, Department 32-24, EB 1300 N. Dort Highway

Flint MI 48556

David O. Kennedy

Lester B. Knight Cast Metals Inc.

549 W. Randolph Street

Chicago IL 60661

George Keros

Photon Physics

3175 Penobscot Building

Detroit MI 48226

Pramod K. Khandelwal

General Motors Corporation

Allison Gas Turbine Division

P.O. Box 420, MS:W05

Indianapolis IN 46206

Jim R. Kidwell

AlliedSignal, Inc.

Auxiliary Power

P.O. Box 52180

Phoenix AZ 85072-2180

Shin Kim

Korea Institute of Machinery \& Metals 66 Sangnam-dong, Changwon

Kyungnam 641-010

KOREA

W. C. King

Mack Truck, Z-41

1999 Pennsylvania Avneue

Hagerstown MD 21740

Carol Kirkpatrick

MSE, Inc.

P.O. Box 3767

Butte MT 59702

Tony Kirn

Caterpillar Inc.

Defense Products Department, JB7

Peoria IL 61629
James D. Kiser

NASA Lewis Research Center

21000 Brookpark Road, MS:49-3

Cleveland OH 44135

Max Klein

900 24th Street, N.W., Unit G

Washington DC 20037

Richard N. Kleiner

Coors Ceramics Company

4545 McIntyre Street

Golden CO 80403

Stanley J. Klima

NASA Lewis Research Center

21000 Brookpark Road, MS:6-1

Cleveland $\mathrm{OH} 44135$

Chris E. Knapp

Norton Advanced Ceramics of Canada Ltd.

8001 Daly Street

Niagara Falls Ontario L2G 6S2

CANADA

Albert S. Kobayashi

University of Washington

Mechanical Engineering Department

Mail Stop:FU10

Seattle WA 98195

Shigeki Kobayashi

Toyota Central Research Labs, Inc.

Nagakute Aichi 480-11

JAPAN

Richard A. Kole

Z-Tech Corporation

8 Dow Road

Bow NH 03304

E. Kostiner

University of Connecticut

Chemistry Department, U-60

Storrs CT 06269-3060 
Joseph A. Kovach

Eaton Corporation

32500 Chardon Road

Willoughby Hills OH 44094

Kenneth A. Kovaly

Technical Insights Inc.

P.O. Box 1304

Fort Lee NJ 07024-9967

Ralph G. Kraft

Spraying Systems Company

North Avenue at Schmale Road

Wheaton IL 60189-7900

Arthur Kranish

Trends Publishing Inc.

1079 National Press Building

Washington DC 20045
A. S. Krieger
Radiation Science, Inc.
P.O. Box 293
Belmont MA 02178
Pieter Krijgsman
Ceramic Design International Holding B.V.
P.O. Box 68
Hattem 8050-AB
THE NETHERLANDS

Waltraud M. Kriven

University of Illinois

105 S. Goodwin Avenue

Urbana IL 61801

Edward J. Kubel, Jr.

ASM International

Advanced Materials \& Processes

Materials Park OH 44073

Dave Kupperman

Argonne National Laboratory

9700 S. Cass Avenue

Argonne IL 60439
Oh-Hun Kwon

North Company

Advanced Ceramics

Goddard Road

Northboro MA 01532-1545

W. J. Lackey

GTRI

Materials Science and Tech. Lab

Atlanta GA 30332

Jai Lala

Tenmat Ltd.

40 Somers Road

Rugby Warwickshire CV22 7DH

ENGLAND

Hari S. Lamba

General Motors Corporation

9301 West 55th Street

LaGrange IL 60525

Richard L. Landingham

Lawrence Livermore National Laboratory

P.O. Box 808, L-369

Livermore CA 94550

Charles J. Landry

Chand Kare Technical Ceramics

712 Flat Hill Road

Lumenburg MA 01462

James Lankford

Southwest Research Institute

6220 Culebra Road

San Antonio TX 78228-0510

Stanley B. Lasday

Business News Publishing Co.

1910 Cochran Road, Suite 630

Pittsburgh PA 15220

Mark S. Laser

Solar Turbines, Inc

2211 Erie Street

San Diego CA 92110 
S. K. Lau

Carborundum Company

Technology Division

P.O. Box 832, B-100

Niagara Falls NY 14302

Edward A. Lauder

Advanced Composite Materials Corporation

1525 S. Buncombe Road

Greer SC 29651-9208

J. Lawrence Lauderdale

Babcock \& Wilcox

1850 "K" Street, Suite 950

Washington DC 20006

Jean F. LeCostaouec

Textron Specialty Materials

2 Industrial Avenue

Lowell MA 01851

Benson P. Lee

Interscience, Inc.

9718 Lake Shore Boulevard

Cleveland $\mathrm{OH} 44108$

Burtrand I. Lee

Clemson University

Olin Hall

Clemson SC 29634-0907

June-Gunn Lee

KIST

P.O. Box 131, Cheong-Ryang

Seoul $130-650$

KOREA

Ran-Rong Lee

Ceramics Process Systems Corporation

155 Fortune Boulevard

Mildford MA 01757

Stan Levine

NASA Lewis Research Center

21000 Brookpark Road, MS:49-3

Cleveland $\mathrm{OH} 44135$
Alan V. Levy

Lawrence Berkeley Laboratory

One Cyclotron Road, MS:62-203

Berkeley CA 94720

Ai-Kang Li

Materials Research Laboratories, ITRI

195-5 Chung-Hsing Road, Sec. 4

Chutung Hsinchu 31015 R.O.C.

TAIWAN

Winston W. Liang

Hong Kong Industrial Technology Centre

78 Tat Chee Avenue

4/F, HKPC Building -- Kowloon

HONG KONG

Robert Licht

Norton Company

Advanced Ceramics

Goddard Road

Northboro MA 01532-1545

E. Lilley

Norton Company

Advanced Ceramics

Goddard Road

Northboro MA 01532-1545

Chih-Kuang Lin

National Central University

Department of Mechanical Engineering

Chung-Li 32054

TAIWAN

Laura J. Lindberg

AlliedSignal Aerospace Company

Garrett Fluid Systems Division

P.O. Box 22200

Tempe AZ 85284-2200

Hans A. Lindner

Cremer Forschungsinstitut GmbH\&Co.KG

Oeslauer Strasse 35

D-8633 Rodental 8866

GERMANY 
Ronald E. Loehman

Sandia National Laboratories

Chemistry \& Ceramics Department 1840

P.O. Box 5800

Albuquerque NM 87185

Jeffrey C. Logas

Winona State University

115 Pasteur Hall

Winona MN 55987

Bill Long

Babcock \& Wilcox

P.O. Box 11165

Lynchburg VA 24506

William D. Long

Wacker Chemicals (USA), Inc.

ESK Engineered Ceramics

50 Locust Avenue

New Canaan CT 06840

L. A. Lott

EG\&G Idaho, Inc.

Idaho National Engineering Laboratory

P.O. Box 1625

Idaho Falls ID 83415-2209

Raouf O. Loutfy

MER Corporation

7960 S. Kolb Road

Tucson AZ 85706

Gordon R. Love

Aluminum Company of America

Alcoa Technical Center

Alcoa Center PA 15960

Lydia Luckevich

Ortech International

2395 Speakman Drive

Mississauga Ontario L5K 1B3

CANADA
James W. MacBeth

Carborundum Company

Structural Ceramics Division

P.O. Box 1054

Niagara Falls NY 14302

H. MacLaren

General Electric Conipany

1000 Western Avenue

Lynn MA 01910

George Maczura

Aluminum Company of America

3450 Park Lane Drive

Pittsburgh PA 15275-1119

David Maginnis

Tinker AFB

OC-ALC/LIIRE

Tinker AFB OK 73145-5989

Frank Maginnis

Aspen Research, Inc.

220 Industrial Boulevard

Moore OK 73160

Tai-il Mah

Universal Energy Systems, Inc.

4401 Dayton-Xenia Road

Dayton OH 45432

Kenneth M. Maillar

Barbour Stockwell Company

83 Linskey Way

Cambridge MA 02142

S. G. Malghan

NIST

I-270 \& Clopper Road

Gaithersburg MD 20899

Lars Malmrup

United Turbine $A B$

Box 13027

Malmo S-200 44

SWEDEN 
John Mangels

Ceradyne, Inc.

3169 Redhill Avenue

Costa Mesa CA 92626

Murli Manghnani

University of Hawaii

2525 Correa Road

Honolulu HI 96822

Russell V. Mann

Matec Applied Sciences, Inc.

75 South Street

Hopkinton MA 01748

William R. Manning

Champion Aviation Products Division

P.O. Box 686

Liberty SC 29657

Ken Marnoch

Amercom, Inc.

8928 Fullbright Avenue

Chatsworth CA 91311

Robert A. Marra

Aluminum Company of America

Alcoa Technical Center

Alcoa Center PA 15069

Chauncey L. Martin

3M Company

3M Center, Building 60-1N-01

St. Paul MN 55144

Steve C. Martin

Advanced Refractory Technologies, Inc.

699 Hertel Avenue

Buffalo NY 14207

Kelly J. Mather

William International Corporation

2280 W. Maple Road

Walled Lake MI 48088
James P. Mathers

3M Company

3M Center, Bldg. 201-3N-06

St. Paul MN 55144

Ron Mayville

Arthur D. Little, Inc.

15-163 Acorn Park

Cambridge MA 02140

F. N. Mazadarany

General Electric Company

Bldg. K-1, Room MB-159

P.O. Box 8

Schenectady NY 12301

James W. McCauley

Alfred University

Binns-Merrill Hall

Alfred NY 14802

Louis R. McCreight

2763 San Ramon Drive

Rancho Palos Verdes CA 90274

Colin F. McDonald

McDonald Thermal Engineering

1730 Castellana Road

La Jolla CA 92037

B. J. McEntire

Norton Company

TRW Ceramics

Goddard Road

Northboro MA 01532-1545

Chuck McFadden

Coors Ceramics Company

600 9th Street

Golden CO 80401

Thomas D. McGee

Iowa State University

110 Engineering Annex

Ames IA 50011 
Carol MicGill

Corning Inc.

Sullivan Park, FR-02-08

Corning NY 14831

James McLaughlin

Sindstrand Power Systems

\400 Ruffin Road

P.O. Box 85757

San Diego CA 92186-5757

Mait McMonigle

U.S. Department of Energy

Imiproved Energy Productivity Division

CE-231, Forrestal Building

Washington DC 20585

J. C. McVickers

AlliedSignal, Inc.

Auxiliary Fower, Bldg. 1303-206

P.O. Rnu 52180, MS:9317-2

Phoenix AZ 85072-2180

I. B. Meadov'croft

"Ju'?," The Kidgeway

Oxshott

Leatherhead Surrey KT22 OLG

UNITED KIN $=D O M$

Joseph J. Meindl

Reynolds International, Inc.

6603 W. Broad Street

P.O. Box 27002

Richmond VA 23261-7003

Michael D. Meiser

AlliedSignal, Inc.

Ceramic Components

P.O. Box 2960, MS:T21

Torrance CA $90509-2960$

George Messenger

National Research Council of Canada

Building M-7

Ottawa Ontario K1A OR6

CANADA
D. Messier

U.S. Army Materials Technology Lah

SLCMT-EMC

405 Arsenal Street

Watertown MA 02172-0001

Arthur G. Metcalfe

Arthur G. Metcalfe and Associates, Inc.

2108 East 24th Street

National City CA 91950

R. Metselaar

Eindhoven University

P.O. Box 513

Endhoven $5600 \mathrm{MB}$

THE NETHERLANDS

David J. Michael

Harbison-Walker Refractories Company

P.O. Box 98037

Pittsburgh PA 15227

Ken Michaels

Chrysler Motors Corporation

P.O. Box 1118, CIMS:418-17-09

Detroit MI 48288

Bernd Michel

Institute of Mechanics

P.O. Box 408

D-9010 Chemnitz

GERMANY

David E. Miles

Commission of the European Communities

rue de la Loi, 200

B-1049 Brussels

BELGIUM

Carl E. Miller

AC Rochester

1300 N. Dort Highway, MS:32-3i

Flint MI 48556

Charles W. Miller, Jr.

Centorr Furnaces/Vacuum Industries

542 Amherst Street

Nashua NH 03063 
R. Minimmi

Enichem America

2000 Cornwall Road

Monmcuth Junction NJ 08852

Michele V. Mitchell

AlliedSignal, Inc.

Ceramic Components

P.O. Box 2960, MS:T21

Torrance CA 90509-2960

Howard Mizuhara

WESGO

477 Harbor Boulevard

Belmont CA 94002

Helen Moeller

Babcock \& Wilcox

P.O. Box 11165

Lynchburg VA 24506-1165

Francois R. Mollard

Concurrent Technologies Corporation 1450 Scalp Avenue

Johnstown PA 15904-3374

Phil Mooney

Panametrics

221 Crescent Street

Waltham MA 02254

Geoffrey P. Morris

3M Company

3M Traffic Control Materials Division

Bldg. 209-BW-10, 3M Center

St. Paul MN 55144-1000

Jay A. Morrison

Rolls-Royce, Inc.

2849 Paces Ferry Road, Suite 450

Atlanta GA 30339-3769

Joel P. Moskowitz

Ceradyne, Inc.

3169 Redhill Avenue

Costa Mesa CA 92626
Brij Moudgil

University of Florida

Material Science \& Engineering

Gainesville FL 32611

Christoph J. Mueller

Sprechsaal Publishing Group

P.O. Box 2962, Mauer 2

D-8630 Coburg

GERMANY

Thomas W. Mullan

Vapor Technologies Inc.

345 Route 17 South

Upper Saddle River NJ 07458

M. K. Murthy

MkM Consultants International

10 Avoca Avenue, Unit 1906

Toronto Onntario M4T 2B7

CANADA

David L. Mustoe

Custom Technical Ceramics

8041 West I-70 Service Road, Unit 6

Arvada CO 80002

Curtis V. Nakaishi

U.S. Department of Energy

Morgantown Energy Technology Center

P.O. Box 880

Morgantown WV 26507-0880

Yoshio Nakamura

Faicera Research Institute

3-11-12 Misono

Sagamihara, Tokyo

JAPAN

K. S. Narasimhan

Hoeganaes Corporation

River Road

Riverton NJ 08077

Samue! Natansohn

GTE Laboratories, Inc.

40 Sylvan Road

Waltham MA 02254 
Robert Naum

Applied Resources, Inc.

P.O. Box 241

Pittsford NY 14534

Malcolm Naylor

Cummins Engine Company, Inc.

P.O. Box 3005, Mail Code 50183

Columbus IN 47202-3005

Jeffrey T. Neil

GTE Laboratories, Inc.

40 Sylvan Road

Waltham MA 02254

Fred A. Nichols

Argonne National Laboratory

9700 S. Cass Avenue

Argonne IL 60439

H. Nickel

Forschungszentrum Juelich (KFA)

P.O. Box 1913

Juelich 1-5170 BRD NRW

GERMANY

Dale E. Niesz

Rutgers University

Center for Ceramic Research

P.O. Box 909

Piscataway NJ 08855-0909

David M. Nissley

United Technologies Corporation

Pratt \& Whitney Aircraft

400 Main Street, MS:163-10

East Hartford CT 06108

Bruce E. Novich

Ceramics Process Systems Corporation

155 Fortune Boulevard

Milford MA 01757

Daniel Oblas

50 Meadowbrook Drive

Bed́ford MÁ 01730
Don Ohanehi

Magnetic Bearings, Inc.

1908 Sussex Road

Blacksburg VA 24060

Robert Orenstein

General Electric Company

55-112, River Road

Schenectady NY 12345

Norb Osborn

Aerodyne Dallas

151 Regal Row, Suite 120

Dallas TX 75247

N. I. Paille

Norton Company

Advanced Ceramics

Goddard Road

Northboro, MA 01532-1545

Richard Palicka

Cercom, Inc.

1960 Watson Way

Vista CA 92083

Muktesh Paliwal

GTE Products Corporation

Hawes Street

Towanda PA 18848

Joseph E. Palko

General Electric Company

55-113, River Road

Schenectady NY 12345

Joseph N. Panzarino

Saint-Gobain/Norton Industrial Ceramics

Northboro Research Center

Goddard Road

Northboro MA 01532-1545

Pellegrino Papa

Corning Inc.

MP-WX-02-1

Coming NY 14831

Terry Paquet

Boride Products Inc.

2879 Aero Park Drive

Traverse City MI 49684 
E. Beth Pardue

MPC

8297 Williams Ferry Road

Lenior City. TN 37771

Soon C. Park

3M Company

Building $142-4 \mathrm{~N}-02$

P.O. Box 2963

St. Paul MN 55144

Harmut Paschke

Schott Glaswerke

Christoph-Dorner-Strasse 29

D-\&300 Landshut

GERMANY

James W. Patten

Cummins Engine Company, Inc.

P.O. Box 3005, Mail Code 50183

Columbus IN 47202-3005

P. J. Pelletier

Norton Company

Advanced Ceramics

Goddard Road

Northboro, MA 01532-1545

Robert A. Penty

Eastman Kodak Company

901 Elmgrove Road

Rochester NY 14653

Robert W. Pepper

Textron Specialty Materials

2 Industrial Avenue

Lowell MA 01851

Peter Perdue

Detroit Diesel Corporation

13400 Outer Drive West, Speed Code L-04

Detroit MI 48239-4001

Bruce Peters

Dow Chemical Company

Building 52

Midland MI 48667
John J. Petrovic

Los Alamos National Laboratory

Group MST-4, MS:G771

Los Alamos NM 87545

Frederick S. Pettit

! University of Pittsburgh

Pittsburgh PA 15261

Ben A. Phillips

Phillips Engineering Company

721 Pleasant Street

St. Joseph MI 49085

Richard C. Phoenix

Ohmtek, Inc.

2160 Liberty Drive

Niagara Falls NY 14302

Bruce J. Pletka

Michigan Technological University

Metallurgical \& Materials Engineering

Houghton MI 49931

John P. Pollinger

AlliedSignal, Inc.

Ceramic Components

P.O. Box 2960, MS:T21

Torrance CA 90509-2960

P. Popper

High Tech Ceramics International Journal

22 Pembroke Drive - Westlands

Newcastle-under-Lyme Staffs ST5 2JN

ENGLAND

F. Porz

Universitat Karlsruhe

Institut fur Keramik Im Maschinendau

Postfach 6980

D-7500 Karlsruhe

GERMANY

Harry L. Potma

Royal Netherlands Embassy

Science and Technology

4200 Linnean Avenue, N.W.

Washington DC 20068 
Bob R. Powell

General Motors Research Laboratories Metallurgy Department

Box 9055

Warren MI 48090-9055

Stephen C. Pred

ICD Group, Inc.

1100 Valley Brook Avenue

Lyndhurst NJ 07071

Karl M. Prewo

United Technologies Research Center

411 Silver Lane, MS:24

East Hartford CT 06108

Peter E. Price

Industrial Materials Technology, Inc.

P.O. Box 9565

Andover MA 01810

Joseph M. Proud

GTE Laboratories, Inc.

40 Sylvan Road

Waltham MA 02254

Vimal K. Pujari

Norton Company

Advanced Ceramics

Goddard Road

Northboro MA 01532-1545

George Quinn

NIST

Ceramics Division, Bldg. 223

Gaithersburg MD 20899

Ramas V. Raman

Ceracon, Inc.

1101 N. Market Boulevard, Suite 9

Sacramento CA 95834

Charles F. Rapp

Owens Corning Fiberglass

2790 Columbus Road

Granville OH 43023-1200
Dennis W. Readey

Colorado School of Mines

Metallurgy and Materials Engineering

Golden CO 80401

Wilfred J. Rebello

PAR Enterprises, Inc.

12601 Clifton Hunt Lane

Clifton VA 22024

Harold Rechter

Chicago Fire Brick Company

7531 S. Ashland Avenue

Chicago IL 60620

Robert R. Reeber

U.S. Army Research Office

P.O. Box 12211

Research Triangle Park NC 27709

K. L. Reifsnider

VPI \& SU

Engineering Science and Mechanics

Blacksburg VA 24061

Paul E. Rempes

McDonnell Douglass Aircraft Company

P.O. Box 516, Mail Code:0642263

St. Louis MO 63166-0516

Gopal S. Revankar

John Deere Company

3300 River Drive

Moline IL 61265

K. Y. Rhee

Rutgers University

P.O. Box 909

Piscataway NJ 08854

James Rhodes

Advanced Composite Materials Corporation 1525 S. Buncombe Road

Greer SC 29651 
Roy W. Rice

W. R. Grace and Company

7379 Route 32

Columbia MD 21044

David W. Richerson

2093 E. Delmont Drive

Salt Lake City UT 84117

Tomas Richter

J. H. France Refractories

1944 Clarence Road

Snow Shoe PA 16874

Michel Rigaud

Ecole Polytechnique

Campus Universite De Montreal

P.O. Box 6079, Station A

Montreal, P.Q. Quebec H3C 3A7

CANADA

Barry Ringstrom

Superior Graphite Company

P.O. Box 2373

Smyrna GA 30081

John E. Ritter

University of Massachusetts

Mechanical Engineering Department

Amherst MA 01003

Frank L. Roberge

AlliedSignal, Inc.

Auxiliary Power

P.O. Box 52180

Phoenix AZ 85072-2180

W. Eric Roberts

Advanced Ceramic Technology, Inc.

990 "F" Enterprise Street

Orange CA 92667

Y. G. Roman

TNO TPD Keramick

P.O. Box 595

Einhoven 5600 ÁN

HOLLAND

Michael Rossetti

Arthur D. Little, Inc.

15 Acorn Park

Cambridge MA 01240
Barry Rossing

Lanxide Corporation

P.O. Box 6077

Newark DE 19714-6077

Steven L. Rotz

Lubrizol Corporation

29400 Lakeland Boulevard

Wickliffe OH 44092

Bruce Rubinger

Global Competitiveness, Inc.

One Devonshire Place, Suite 1011

Boston MA 02109

Robert Ruh

Wright Laboratory

WL/MLLM

Wright-Patterson AFB OH 45433

Robert J. Russell

17 Highgate Road

Framingham MA 01701

L. William Sahley

Supermaterials Company

24400 Highland Road

Richmond Heights OH 44143

Jon A. Salem

NASA Lewis Research Center

21000 Brookpark Rcdd

Cleveland OH 44135

L. C. Sales

Norton Company

Advanced Ceramics

Goddard Road

Northboro, MA 01532-1545

W. A. Sanders

NASA Lewis Research Center 21000 Brookpark Road, MS:49-3

Cleveland $\mathrm{OH} 44135$

J. Sankar

North Carolina A\&T State University

Department of Mechanical Engineering

Greensboro NC 27406 
Yasushi Sato

NGK Spark Plugs (U.S.A.), Inc. 1200 Business Center Drive, Suite 300

Mt. Prospect IL 60056

Maxine L. Savitz

AlliedSignal, Inc.

Ceramic Components

P.O. Box 2960, MS:T21

Torrance CA 90509-2960

Ashok Saxena
GTRI
Materials Engineering
Atlanta GA 30332-0245
David W. Scanlon
Instron Corporation
100 Royall Street
Canton MA 02021
Charles A. Schacht
Schacht Consulting Services
12 Holland Road
Pittsburgh PA 15235
James Schienle
AlliedSignal, Inc.
Auxiliary Power
P.O. Box 52180, MS:1302-2P
Phoenix AZ 85072-2180

John C. Schneider

San Juan Technologies

P.O. Box 49326

Colorado Springs CO 80949-9326

Gary Schnittgrund

Rocketdyne, BA05

6633 Canoga Avenue

Canoga Park CA 91303

Mark Schomp

Lonza, Inc.

17-17 Route 208

Fâii Laññ Ny 07410
Joop Schoonman

Delft University of Technology

P.O. Box 5045

2600 GA Delft

THE NETHERLANDS

John Schuldies

Industrial Ceramic Technology, Inc.

30 Orchard Way, North

Potomac MD 20854

Robert B. Schulz

U.S. Department of Energy

Office of Transportation Materials

CE-34, Forrestal Building

Washington DC 20585

Murray A. Schwartz

Materials Technology Consulting Inc.

30 Orchard Way, North

Potomac MD 20854

Peter Schwarzkopf

SRI International

333 Ravenswood Avenue

Menlo Park CA 94025

William T. Schwessinger

Multi-Arc Scientific Coatings

1064 Chicago Road

Troy MI 48083-4297

W. D. Scott

University of Washir.gton

Materials Science Department

Mail Stop:FB10

Seattle WA 98195

Nancy Scoville

Thermo Electron Technologies

P.O. Box 9046

Waltham MA 02254-9046

Thomas M. Sebestyen

U.S. Department of Energy

Advanced Propulsion Division

CE-322, Forrestal Building

Washington DC 20585 
Brian Seegmiller

Coors Ceramics Company

600 9th Street

Golden CO 80401

T. B. Selover

AICRE/DIPPR

3575 Traver Road

Shaker Heights OH 44122

J. H.. Selverian

GTE Laboratories, Inc.

40 Sylvan Road

Waltham MA 02254

Charles E. Semler

Semler Materials Services

4160 Mumford Court

Columbus OH 43220

Thomas Service

Service Engineering Laboratory

324 Wells Street

Greenfield MA 01301

Kish Seth

Ethyl Corporation

P.O. Box 341

Baton Rouge LA 70821

Karleen Seybold

AlliedSignal, Inc.

Auxiliary Power

P.O. Box 52180

Phoenix AZ 85072-2180

William J. Shack

Argonne National Laboratory

9700 S. Cass Avenue, Bldg. 212

Argonne IL 60439

Peter T.B. Shaffer

Technical Ceramics Laboratories, Inc. 4045 Nine/McFarland Drive

Alpharetta GA 30201
Richard K. Shaltens

NASA Lewis Research Center

21000 Brookpark Road, MS:302-2

Cleveland $\mathrm{OH} 44135$

Robert S. Shane

1904 NW 22nd Street

Stuart FL 34994-9270

Ravi Shankar

Chromalloy

Research and Technology Division

Blaisdell Road

Orangeburg NY 10962

Terence Sheehan

Alpex Wheel Company

727 Berkley Street

New Milford NJ 07646

Dinesh K. Shetty

University of Utah

Materials Science and Engineering

Salt Lake City UT 84112

Masahide Shimizu

Ceramic Society of Japan

2-2-503 Takiyama 6-chome

Higashikurume-Shi Tokyo 203

JAPAN

John Shipinski

Toyota Technical Center U.S.A., Inc.

Technical Research Department

2000 Town Center, Suite 500

Southfield MI 48075

Thomas Shreves

American Ceramic Society, Inc.

735 Ceramic Place

P.O. Box 6136

Westerville $\mathrm{OH} 43081-8720$

Jack D. Sibold

Coors Ceramics Company

4545 McIntyre Street

Golden CO 80403 
Johann Siebels

Volkswagen AG

Werkstofftechnologie

3180 Wolfsburg 1

GERMANY

George H. Siegel

Point North Associates, Inc.

P.O. Box 907

Madison NJ 07940

Richard Silberglitt

Technology Assessment and Transfer, Inc.

133 Defense Highway, \#212

Annapolis MD 21401

Mary Silverberg

Norton Company

Goddard Road

Northboro MA 01532-1545

Gurpreet Singh

Department of the Navy

Code 56X31

Washington DC 20362-5101

Maurice J. Sinnott

University of Michigan

5106 IST Building

Ann Arbor MI 48109-2099

John Skildum

3M Company

3M Center

Building 224-2S-25

St. Paul MN 55144

David P. Smith

Hoskins, Rees \& Smith

4405 Steubenville Pike

Pittsburgh PA 15205-9632

Richard H. Smoak

Smoak \& Associates

3554 Hollyslope Road

Altadena CA 91001-3923
Jay R. Smyth

AlliedSignal, Inc.

Auxiliary Power

P.O. Box 52180, MS:93-173/1303-207

Phoenix AZ 85072-2180

Rafal A. Sobotowski

British Petroleum Company

Technical Center, Broadway

3092 Broadway Avenue

Cleveland $\mathrm{OH} 44115$

A. G. Solomah

SAC International Ltd.

1445 Bonhill Road, \#13

Mississauga Ontario L5T 1V3

CANADA

S. Somiya

Nishi Tokyo University

3-7-19 Seijo, Setagaya

Tokyo 157

JAPAN

Boyd W. Sorenson

DuPont Lanxide Composites

1300 Marrows Road

Newark DE 19711

Charles A. Sorrell

U.S. Department of Energy

Advanced Industrial Concepts Division

CE-232, Forrestal Building

Washington DC 20585

C. Spencer

EA Technology

Capenhurst Chester CH1 6ES

UNITED KINGDOM

Allen Spizzo

Hercules Inc.

Hercules Plaza

Wilmington DE 19894 
Richard M. Spriggs

Alfred University

Center for Advanced Ceramic Technology

Alfred NY 14802

Charies Spuckler

NASA Lewis Research Center

21000 Brookpark Road, MS:5-11

Cleveland OH 44135-3191

M. Srinivasan

Material Solutions

P.O. Box 663

Grand Island NY 14702-0663

Gordon L. Starr

Cummins Engine Company, Inc.

P.O. Box 3005, Mail Code:50182

Columbus IN 47202-3005

Jim Stevenson

Windrock, Inc.

835 Innovation Drive

Knoxville TN 37932

Tom Stillwagon

AlliedSignal, Inc.

Ceramic Components

P.O. Box 2960, MS:T21

Torrance CA 90509-2960

H. M. Stoller

TPL Inc.

3754 Hawkins, N.E.

Albuquerque NM 87109

Paul D. Stone

Dow Chemical USA

1776 "Eye" Street, N.W., Suite 575

Washington DC 20006

Roger S. Storm

Carborundum Company

Technology Division

P.O. Box 337

Niagara Falls NY 14302-0337
F. W. Stringer

Aero \& Industrial Technology Ltd.

P.O. Box 46, Wood Top

Burnley Lancashire BB11 4BX

UNITED KINGDOM

Thomas N. Strom

NASA Lewis Research Center

21000 Brookpark Road, MS:86-6

Cleveland $\mathrm{OH} 44135$

M. F. Stroosnijder

Institute for Advanced Materials

Joint Research Centre

21020 Ispra (VA)

ITALY

Karsten Styhr

30604 Ganado Drive

Rancho Palos Verdes CA 90274

T. S. Sudarshan

Materials Modification, Inc.

2929-P1 Eskridge Center

Fairfax VA 22031

M. J. Sundaresan

University of Miami

P.O. Box 248294

Coral Gables FL 33124

Patrick L. Sutton

U.S. Department of Energy

Office of Propulsion Systems

CE-322, Forrestal Building

Washington DC 20585

Willard H. Sutton

United Technologies Corporation

Silver Lane, MS:24

East Hartford CT 06108

J. J. Swab

U.S. Army Materials Technology Lab

Ceramics Research Division, SLCMT-EMC

405 Arsenal Street

Watertown MA 02172 
Robert E. Swanson

Metalworking Technology, Inc.

1450 Scalp Avenue

Johnstown PA 15904

Scott L. Swartz

BCL

Metals and Ceramics

505 King Avenue

Columbus OH 43201

Steve Szaruga

Air Force Wright Aeronautical Lab

WL/MLBC

Wright-Patterson AFB OH 45433-6533

Yo Tajima

NGK Spark Plug Company

2808 Iwasaki

Komaki-shi Aichi-ken 485

JAPAN

Fred Teeter

5 Tralee Terrace

East Amherst NY 14051

Monika O. Ten Eyck

Carborundum Company

Technology Division

P.O. Box 832

Niagara Falls NY 14302

David F. Thompson

Corning Glass Works

SP-DV-02-1

Corning NY 14831

Merle L. Thorpe

Hobart Tafa Technologies, Inc.

146 Pembroke Road

Concord NH 03301

T. Y. Tien

University of Michigan

Materials Science and Engineering

Dow Building

Ann Arbor MI 48103
D. M. Tracey

Norton Company

Advanced Ceramics

Goddard Road

Northboro MA 01532-1545

L. J. Trostel, Jr.

Box 199

Princeton MA 01541

W. T. Tucker

General Electric Company

P.O. Box 8, Bldg. K1-4C35

Schenectady NY 12301

Masanori Ueki

Nippon Steel Corporation

1618 Ida

Nakahara-Ku Kawasaki 211

JAPAN

Filippo M. Ugolini

ATA Studio

Via Degli Scipioni, 268A

ROMA, 00192

ITALY

Donald L. Vaccari

General Motors Corporation

Allison Gas Turbines

P.O. Box 420, Speed Code S49

Indianapolis IN 46206-0420

Carl F. Van Conant

Boride Products, Inc.

2879 Aero Park Drive.

Traverse City MI 49684

Marcel H. Van De Voorde

Commission of the European Communities

P.O. Box 2

1755 ZG Petten

THE NETHERLANDS 
O. Van Der Biest

Katholieke Universiteit Leuven

Departement Metaalkunde en Toegepaste de Croylaan 2

B-3030 Leuven

BELGIUM

Michael Vannier

Washington University, St. Louis

510 S. Kings Highway

St. Louis MO 63110

Stan Venkatesan

Southern Coke \& Coal Corporation

P.O. Box 52383

Knoxville TN 37950

V. Venkateswaran

Carborundum Company

Niagara Falls R\&D Center

P.O. Box 832

Niagara Falls NY 14302

Dennis Viechnicki

U.S. Army Materials Technology Lab

405 Arsenal Street

Watertown MA 02172-0001

Ted Vojnovich

U.S. Department of Energy

Office of Energy Research, ER-42

Washington DC 20585

John D. Volt

E.I. Dupont de Nemours \& Company, Inc.

P.O. Box 80262

Wilmington DE 19880

John B. Wachtman

Rutgers University

P.O. Box 909

Piscataway NJ 08855

Shigetaka Wada

Toyota Central Research Labs, Inc.

Nagakute Aichi 480-11

JAPAN
Janet Wade

AlliedSignal, Inc.

Auxiliary Power

P.O. Box 52180, MS:1303-2

Phoenix AZ 85072-2180

Richard L. Wagner

Ceramic Technologies, Inc.

537 Turtle Creek South Drive, Suite 24D

Indianapolis IN 46227

J. Bruce Wagner, Jr.

Arizona State University

Center for Solid State Science

Tempe AZ 85287-1704

Daniel J. Wahlen

Kohler, Co.

444 Highland Drive

Kohler WI 53044

Ingrid Wahlgren

Royal Institute of Technology

Studsvik Library

S-611 82 Nykoping

SWEDEN

Ron H. Walecki

AlliedSignal, Inc.

Ceramic Components

P.O. Box 2960, MS:T21

Torrance CA 90509-2960

Michael S. Walsh

Vapor Technologies Inc.

2100 Central Avenue

Boulder CO 80301

Chien-Min Wang

Industrial Technology Research Institute

195 Chung-Hsing Road, Sec. 4

Chutung Hsinchu 31015 R.O.C.

TAIWAN

Robert M. Washburn

ASMT

11203 Colima Road

Whittier CA 90604 
Gerald Q. Weaver

Carborundum Specialty Products

42 Linus Allain Avenue

Gardner MA 01440-2478

Kevin Webber

Toyota Technical Center, U.S.A. Inc.

1410 Woodridge, RR7

Ann Arbor MI 48105

Karen E. Weber

Detroit Diesel Corporation

13400 Outer Drive West

Detroit MI 48239-4001

J. K. Weddell

DuPont Fibers

Experimental Station, E302

Wilmington DE 19880-0302

R. W. Weeks

Argonne National Laboratory

MCT-212

9700 S. Cass Avenue

Argonne IL 60439

Ludwig Weiler

ASEA Brown Boveri AG

Eppelheimer Str. 82

D-6900 Heidelberg

GERMANY

James Wessel

Dow Corning Corporation

1800 "M" Street, N.W., Suite 325 South

Washington DC 20036

Robert D. West

Therm Advanced Ceramics

P.O. Box 220

Ithaca NY 14851

Thomas J. Whalen

Ford Motor Company

26362 Harriet

Dearborn Heights MI 48127
Ian A. White

Hoeganaes Corporation

River Road

Riverton NJ 08077

Alan Whitehead

General Electric Company

1 River Road, 55-111

Schenectady NY 12345

Sheldon M. Wiederhorn

NIST

Building 223, Room 1329

Gaithersburg MD 20899

John F. Wight

Alfred University

McMahon Building

Alfred NY 14802

D. S. Wilkinson

McMaster University

1280 Main Street, West

Hamilton Ontario L8S 4L7

CANADA

James C. Williams

General Electric Company

Engineering Materials Technology Labs

One Neumann Way, Mail Drop:H85

Cincinnati OH 45215-6301

Steve J. Williams

RCG Hagler Bailly, Inc.

1530 Wilson Boulevard, Suite 900

Arlington VA 22209-2406

Thomas A. Williams

National Renewable Energy Laboratory

1617 Cole Boulevard

Golden CO 80401

Craig A. Willkens

Norton Company

Advanced Ceramics

Goddard Road

Northboro MA 01532-1545 
Roger R. Wills

TRW, Inc.

Valve Division

1455 East 185th Street

Cleveland $\mathrm{OH} 44110$

David Gordon Wilson

Massachusetts Institute of Technology

77 Massachusetts Avenue, Room 3-455

Cambridge MA 02139

Matthew F. Winkler

Seaworthy Systems, Inc.

P.O. Box 965

Essex CT 06426

Gerhard Winter

Hermann C. Starck Berlin GmbH

P.O. Box 2540

D-3380 Goslar 3380

GERMANY

W. L. Winterbottom

Ford Motor Company

20000 Rotunda Drive

SRL, Room E-3182

Dearborn MI 48121

William T. Wintucky

NASA Lewis Research Center

Terrestrial Propulsion Office

21000 Brookpark Road, MS:86-6

Cleveland $\mathrm{OH} 44135$

David G. Wirth

Coors Ceramics Company

600 9th Street

Golden CO 80401

Thomas J. Wissing

Eaton Corporation

Engineering and Research Center

P.O. Box 766

Southfield MI 48037
James C. Withers

MER Corporation

7960 S. Kolb Road

Building $\mathrm{F}$

Tucson AZ 85706

Dale E. Wittmer

Southern Illinois University

Mechanical Engineering Department

Carbondale IL 62901.

Warren W. Wolf

Owens Corning Fiberglass

2790 Columbus Road, Route 16

Granville OH 43023

Egon E. Wolff

Caterpillar Inc.

Technical Center

P.O. Box 1875

Peoria IL 61656-1875

George W. Wolter

Howmet Turbine Components Corporation

Technical Center

699 Benston Road

Whitehall MI 49461

James C. Wood

NASA Lewis Research Center

21000 Brookpark Road, MS:86-6

Cleveland $\mathrm{OH} 44135$

Marrill Wood

LECO Corporation

P.O. Box 211688

Augusta GA 30917-1688

Wayne L. Worrell

University of Pennsylvania

3231 Walnut Street

Philadelphia PA 19104

John F. Wosinski

Corning Inc.

ME-2 E-5 H8

Corning NY 14830 
Ian G. Wright

$\mathrm{BCL}$

505 King Avenu:

Columbus $\mathrm{OH} 43201$

F th Wroe

ERDC

Capenhurst Chester CH1 6ES

ENGLAND

Bernard J. Wrona

Advanced Composite Materials Corporation 1525 S. Buncombe Road

Greer SC 29651

Carl C. M. Wu

Naval Research Laboratory

Ceramic Branch, Code 6373

Washington DC 20375

John C. Wurst

University of Dayton Research Institute

300 College Park

Dayton OH 45469-0101

Neil Wyant

ARCH Development Corp.

9700 S. Cass Avenue, Bldg. 202

Argonne IL 60439

Roy Yamamoto

Texaco Inc.

P.O. Box 509

Beacon NY 12508-0509

John Yamanis

AlliedSignal Aerospace Company

P.O. Box 1021

Morristown NJ 07962-1021

R. L. Yeckley

Norton Company

Advanced Ceramics

Goddard Road

Northboro, MA 01532-1545

Harry C. Yeh

AlliedSignal, Inc.

Ceramic Components

P.O. Box 2960, MS:T21

Torrance CA 90509-2960
Hiroshi Yokoyama

Hitachi Research Lab

4026 Kuji-Cho

Hitachi-shi Ibaraki 319-12

JAPAN

Thomas M. Yonushonis

Cummins Engine Company, Inc.

P.O. Box 3005, Mail Code 50183

Columbus IN 47202-3005

Thor' ', J. Yost

Corning Inc.

Technical Products Division, 21-1-2

Corning NY 14831

Jong Yung

Sundstrand Aviation Operations

4747 Harrison Avenue

Rockford IL 61125

A. L. Zadoks

Caterpillar Inc.

Technical Center, Building L

P.O. Box 1875

Peoria IL 61656-1875

Avi Zangvil

University of Illinois

104 S. Goodwin Avenue

Urbana IL 61801

Charles H. Zenuk

Transtech

1340 N. Via Ronda Jeste

Tucson AZ 85715-r330

Carl Zweben

General Electric Company

P.O. Box 8555, VFSC/V4019

Philadelphia PA 19101

Klaus M. Zwilsky

National Research Council

2101 Constitution Avenue

Washington DC 20418 
Office of Assistant Manager for Energy Research and Development

U.S. Department of Energy

Oak Ridge Operations Office

Oak Ridge, TN 37831

U.S. Department of Energy

Office of Scientific and

Technical Information

P.O. Box 62

Oak Ridge, TN 37831 

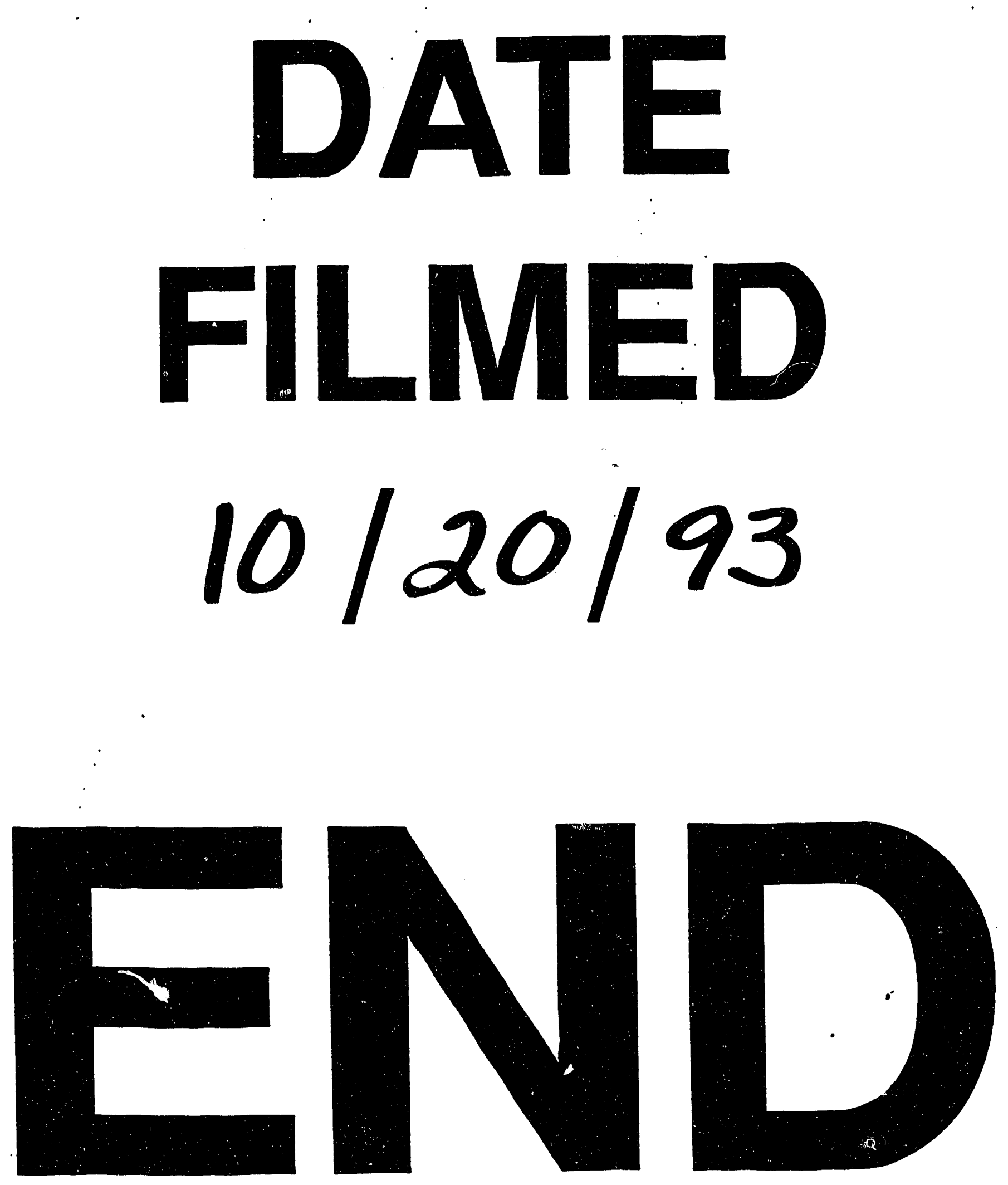
